\title{
Colorectal anastomotic leakage
}

Citation for published version (APA):

Bosmans, J. W. A. M. (2017). Colorectal anastomotic leakage: a call for a different approach. [Doctoral Thesis, Maastricht University]. Datawyse / Universitaire Pers Maastricht.

https://doi.org/10.26481/dis.20170331jb

Document status and date:

Published: 01/01/2017

DOI:

10.26481/dis.20170331jb

Document Version:

Publisher's PDF, also known as Version of record

Document license:

Unspecified

\section{Please check the document version of this publication:}

- A submitted manuscript is the version of the article upon submission and before peer-review. There can be important differences between the submitted version and the official published version of record.

People interested in the research are advised to contact the author for the final version of the publication, or visit the DOI to the publisher's website.

- The final author version and the galley proof are versions of the publication after peer review.

- The final published version features the final layout of the paper including the volume, issue and page numbers.

Link to publication

\footnotetext{
General rights rights.

- You may freely distribute the URL identifying the publication in the public portal. please follow below link for the End User Agreement:

www.umlib.nl/taverne-license

Take down policy

If you believe that this document breaches copyright please contact us at:

repository@maastrichtuniversity.nl

providing details and we will investigate your claim.
}

Copyright and moral rights for the publications made accessible in the public portal are retained by the authors and/or other copyright owners and it is a condition of accessing publications that users recognise and abide by the legal requirements associated with these

- Users may download and print one copy of any publication from the public portal for the purpose of private study or research.

- You may not further distribute the material or use it for any profit-making activity or commercial gain

If the publication is distributed under the terms of Article $25 \mathrm{fa}$ of the Dutch Copyright Act, indicated by the "Taverne" license above, 


\section{Colorectal Anastomotic Leakage A call for a different approach}


(C) Anne-Claire Bosmans, Maastricht 2017

No part of this book may be reproduced or transmitted in any form or by any means, without prior permission in writing by the author, or when appropriate, by the publishers of the publications.

Image on cover: Transversale doorsnede van het colon (PAS-AB, 400x)/Amandelbloesem Elise Vagevuur Goevaerts

ISBN: $\quad 9789461596802$

Layout: Tiny Wouters

Production: Datawyse | Universitaire Pers Maastricht

Publication of this thesis was financially supported by Maastricht University, Department of Surgery of the Maastricht University Medical Center, the Nederlandse Vereniging voor Gastroenterologie (NVGE), and its subdivision Sectie Experimentele Gastroenterologie (SEG). Additional funding was obtained from the Maatschap Heelkunde Zuid-Limburg.

The studies in this thesis were performed at the School of Nutrition and Translational Research in Metabolism (NUTRIM). 


\title{
Colorectal Anastomotic Leakage A call for a different approach
}

\author{
PROEFSCHRIFT \\ ter verkrijging van de graad van doctor aan de Universiteit Maastricht, \\ op gezag van Rector Magnificus prof. dr. Rianne M. Letschert, \\ volgens het besluit van het College van Decanen, \\ in het openbaar te verdedigen \\ op vrijdag 31 Maart 2017 om 14:00 uur \\ door
}

Joanna Wilhelmina Antonia Monique Bosmans

geboren op 3 november 1986

te Tilburg 


\title{
Promotor
}

Prof. dr. N.D. Bouvy

\section{Copromotor}

\author{
Dr. J.P.M. Derikx
}

\section{Beoordelingscommissie}

Prof. dr. L.P.S. Stassen (voorzitter)

Prof. dr. H. Van Goor (Radboud UMC, Nijmegen)

Dr. D.M.A.E. Jonkers

Prof. dr. S.W.M. Olde Damink

Dr. R. Roumen (Máxima Medisch Centrum, Veldhoven) 


\section{Contents}

$\begin{array}{lll}\text { Chapter } 1 & \text { General introduction } & 7\end{array}$

Part I Animal models for research on anastomotic healing 25

Chapter 2 International consensus statement regarding the use of animal 27 models for research on anastomoses in the lower gastrointestinal tract

Chapter 3 MALDI Imaging: a promising tool in elucidating the

pathophysiology of colorectal anastomotic leakage

Part II The use of knockout mice to identify risk factors for anastomotic leakage

Chapter 4 Cyclooxygenase-2 is essential for colorectal anastomotic healing

Chapter 5 Normal mucus composition is crucial in colonic anastomotic healing in mice

Part III Reducing anastomotic leakage rate: glues and enema intervention

Chapter 6 Comparison of three different application routes of butyrate to improve colonic anastomotic strength in rats

Chapter 7 Sealing insufficient colonic anastomoses with cyanoacrylate tissue adhesive: an in vivo study

Part IV Potential disruption of the balance between anastomotic healing and adhesion format

Chapter 8 Effects of glycerol as a plasticizer in biomaterials for intra-abdominal use

Chapter 9 A new poly(1,3-trimethylene carbonate) film provides effective adhesion reduction after major abdominal surgery in a rat model

Chapter 10 General discussion, summary and future perspectives

Valorisation addendum

Dankwoord 



\section{CHAPTER 1}

\section{General introduction and outline of this thesis}

Partly adapted from:

Joanna W.A.M. Bosmans, Audrey C.H.M. Jongen, Nicole D. Bouvy, Joep P.M. Derikx

Colorectal anastomotic healing: why the biological processes that lead to anastomotic leakage should be revealed prior to conducting intervention studies. 
Chapter 1 


\section{Background}

Colorectal cancer (CRC) is the fourth most common cause of cancer death worldwide, estimated to be responsible for 694,000 deaths in $2012^{1}$. The number of CRC patients is concomitantly increasing in the Netherlands due to a higher incidence, population growth, aging of the population and due to the recently established nationwide screening².

Surgery is the predominant curative treatment type for CRC, but has a major impact on the patient's wellbeing by demanding large amounts of metabolic reserves. Subsequently, surgery may lead to the development of frequently observed and severe postoperative complications. Anastomotic leakage (AL) is the most important complication after colorectal surgery and has an incidence of 8$15 \%$ in the Netherlands ${ }^{3}$. AL is associated with a high short-term mortality, with death rates of up to $40 \%{ }^{4,5}$. Although many attempts have been made to prevent AL, thus far none of the proposed interventions have been successful. Despite proper patient selection, reduction of known preoperative risk factors and improved surgical techniques as well as introduction of 'fast track' protocols, incidence of AL has not decreased over the past decade(s).

Furthermore, AL is associated with a decreased disease-specific survival in patients with CRC and an increased recurrence rate of CRC disease ${ }^{4,6-8}$. The necessity to answer the question why some patients develop AL still remains, especially with the current incidence of CRC. Previous work on this topic together with findings from this thesis have led to a large observational study in patients (REVEAL) which aims to establish and validate a diagnostic algorithm for the preoperative prediction of the development of AL using a combination of inflammatory, immune-related and genetic parameters.

\section{Research on anastomotic leakage}

Research on anastomotic leakage can be divided into clinical research and basic (translational) research, often performed in animal models. Basic (and translational) studies on $\mathrm{AL}$ have, for the largest part, been focusing on interventions reducing $\mathrm{AL}$, either by using preventive measures that mechanically supported the anastomosis or by accelerating the healing process of the anastomosis. These interventions have shown to reduce the effects of AL in animal models, but no interventions have proven their value in the clinical setting. Clinical research has predominantly focused on identifying risk factors of $\mathrm{AL}$ and accordingly aimed to eliminating or treat these risk factors. An overview of the pillars of research on anastomotic leakage can be found in Figure 1.1. 


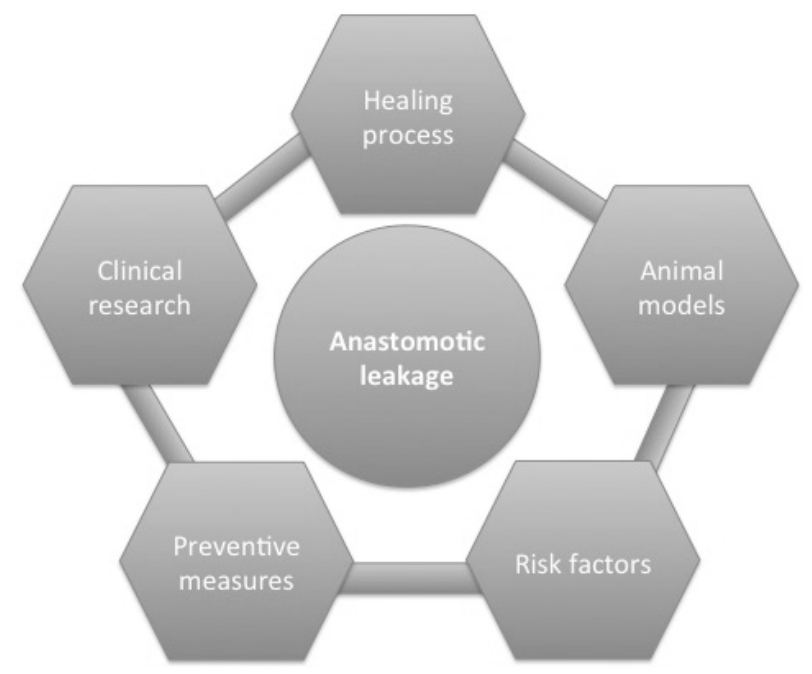

Figure 1.1 Research themes regarding anastomotic leakage.

\section{Animal research on bowel anastomoses}

In an ideal world, the mechanisms of anastomotic healing are investigated in a true clinical model, the patient. In practice however, this is rather difficult except by retrospective analysis. Therefore considerable information comes from knowledge obtained from animal models, despite the known limitations of these models.

Animal research is regulated by international legislation regarding the use of animals in scientific procedures to ensure humane treatment of the animals. There is a great emphasis in the scientific community on minimising use of animals and improving animal welfare. The principles of the 3Rs - Replacement, Reduction and Refinement - are a useful framework for humane animal research and the ARRIVE guidelines aim to improve transparency in animal research together with more comparability between studies ${ }^{9}$.

Replacement can be achieved by using alternative methods, for example in vitro cells or even complete mini-organs, yet this latter technique uses stem cells and is still relatively new and therefore not used on a large scale. Also reduction can be accomplished using new techniques. High resolution-imaging mass spectrometry is such a new technique by which it is possible to visualize the spatial distributions of chemical compositions, such as proteins or metabolites. Since more information is obtained at once with this technique, less tissue and thus fewer animals are required to answer specific research questions. 
The use of animals for studies into (patho)-physiology of bowel anastomoses is highly valuable since numerous study results can be translated to the human setting. Clinical observations can be investigated in depth and standardized in animal models and solutions can be designed to aid patients in the future. However, the quality of animal studies can be significantly improved when researchers become more transparent about their methods, provide detailed information on their models and share their negative results.

If researchers could come to consensus on what is acceptable in animal research and more importantly, what are the best suited animal models, how should randomization happen and what are the minimum requirements to provide enough information regarding animal experiments, this would lead to more reproducibility and comparability between studies. This can also lead to more refined experimental studies, since methods become clear down to the last detail. Unfortunately, publication bias still exists resulting in a discrepancy between the amount of animal experiments performed and the amount actually reported. This results in reperforming studies by different research groups and unnecessary use of animals. Despite the fact that specific journals have been established with a focus on negative results, the scientific community does not yet endorse the importance of publishing these results ${ }^{10}$.

\section{Preventive measures for colorectal anastomotic leakage}

Considerable attempts have been made to prevent AL after colorectal surgery, either by enhancing the healing process through different pharmaceutical approaches, mechanically strengthen the anastomosis or by preventing the sequelae of leakage with specific intraluminal devices. Especially the last two are currently under extensive investigation.

In 2011, Morks and colleagues provided an overview on strategies that prevent or reduce the anastomotic leakage rate by means of a device. Morks divided the devices found in the literature into transanal decompression, intraluminal, and biodegradable protective devices ${ }^{11}$. Generally these devices showed promising results in animals studies, but failed to do so in the clinical setting. Some devices such as the Coloshield seemed to have great potential, but have never been widely accepted $^{11}$.

In the last 5 years, several new devices have been developed to reduce AL. Nevertheless, in current practice not a single of these devices are standardly used during colorectal surgery. Many of these devices work as faecal diverting methods, such as tube ileostomy or transanal tube placement and were successfully tested in several patients ${ }^{12-14}$. A disadvantage of the aforementioned devices is that the tubes 
have to be removed again at some point. Recently, a promising degradable drain was tested in a clinical trial (C-Seal) but this study was stopped preliminary after an interim analysis because there were no significant differences between the intervention group and the control group ${ }^{15}$.

Besides reducing the clinical burden of $\mathrm{AL}$, research has also focused on mechanically supporting anastomoses. One of the most promising tools to enhance colorectal anastomoses seems to be tissue adhesives. Several sorts of tissue adhesives have been tested (see also Chapter 7) and this has led to the conclusion that fibrin-based glues do not have a positive influence on the healing of gastrointestinal anastomoses ${ }^{16}$. Cyanoacrylate-based adhesives however, seem to have a beneficial effect on the mechanical strength ex vivo and a positive influence on anastomotic healing, even in an inflamed animal model ${ }^{17-18}$. These types of tissue adhesives are currently under investigation for gastric varices ${ }^{19}$, but no results have been reported coming from human studies regarding cyanoacrylate and colorectal anastomoses. Furthermore, new compounds were developed - for example based on polyethylene glycol - and have made it through feasibility studies in humans ${ }^{20}$. This specific area within the field of research on colorectal anastomosis is emerging and time will tell if these promising results will lead to a significant change in daily practice.

There are several risks related to the use of tissue adhesives. It is known that tissue adhesives can worsen morbidity and mortality caused by a severe foreign body reaction and increased fibrosis at the anastomotic site 21 . This reaction can interfere with the balance between collagen synthesis and lysis, the so-called collagenous equilibrium that is critical to anastomotic repair ${ }^{22}$. A simplified model of this balance between collagen synthesis and lysis is depicted in Figure 1.2. In addition, tissue adhesives can cause adhesions to the anastomotic site from surrounding fat tissue or intestines, which in turn can lead to mechanical obstruction and subsequent ileus. Complications related to postoperative adhesion formation are frequent, have a large negative effect on patients' health, and increase workload in clinical practice ${ }^{23}$. Therefore, several prophylactic measures have been developed to prevent adhesion formation ${ }^{24}$. Again, these anti-adhesive agents have not entered widespread clinical use because of concerns regarding possible interference with anastomotic healing ${ }^{25}$. Hence, the effect of prophylactic measures for adhesion prevention on anastomotic healing should always be tested.

\section{Discussion points in anastomotic healing}

While there is an on-going increase in experimental studies being published regarding $\mathrm{AL}$, the majority of these articles lack in providing details on anastomotic healing. Moreover, the study endpoints mainly provide information on 1) a 
reduction in $\mathrm{AL}$ rate or 2) an increase in bursting pressure. Although these endpoints are generally accepted in the literature, we wonder if these outcome measures truly are surrogate markers for anastomotic healing. Furthermore, numerous researchers draw direct parallels between anastomotic healing and cutaneous wound healing, but are these processes indeed comparable or should they be considered as two separate physiological processes?

Additionally, it is difficult to come up with new treatment strategies or preventive measures in order to decrease AL when the pathophysiology of anastomotic healing is largely unknown. The focus of research on bowel anastomoses should therefore be redirected from prophylactic interventions tested on animals towards studies that unravel the processes of normal anastomotic healing. This should include identification of essential factors and possible deficiencies in these factors that cause disruption of the anastomotic healing process, and consequently $\mathrm{AL}$.

To date, no consensus has been reached among researchers on 1) which layer of the bowel wall is most important in anastomotic healing, 2) if gastrointestinal healing can be compared to cutaneous healing, 3) if bacteria play a role in the pathogenesis of anastomotic leakage, and 4) if surrogate markers truly provide information regarding anastomotic healing, probably due to a lack of knowledge on these subjects. The majority of experienced surgeons and scientists believe that the most common cause of AL is still unknown ${ }^{26}$; resulting in on-going research using several animal models. In our view this is not appropriate since an insightful understanding of the molecular and biochemical pathways of intestinal anastomotic healing is crucial before animal studies with evidence-based interventions to prevent AL can be initiated.

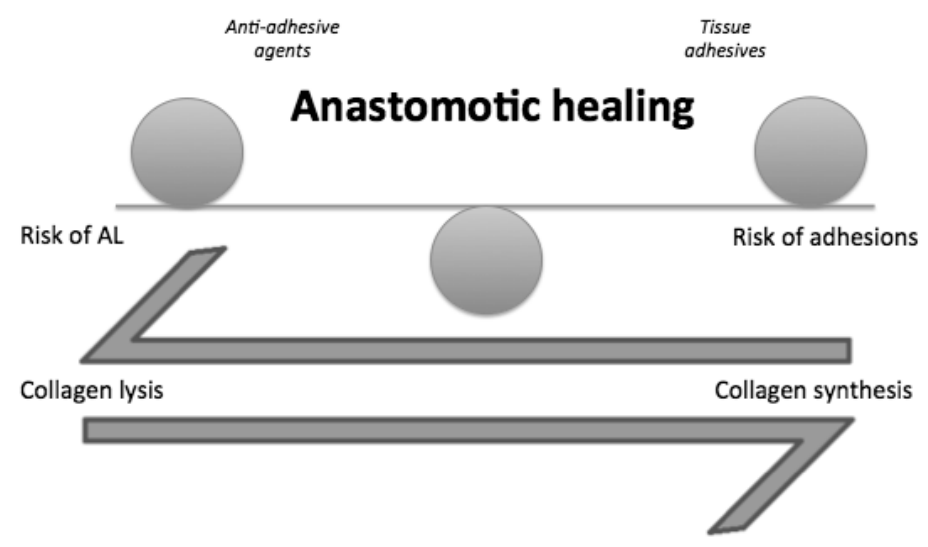

Figure 1.2 Collagenous equilibrium that is critical for anastomotic healing. 


\section{The role of different tissue layers in anastomotic healing}

The bowel wall of the colon consists of four layers: mucosa, submucosa, muscularis propria and serosa. Whenever a colorectal resection is performed, all these layers are transsected and an anastomosis can be constructed. Although surgeons in the past opted for suturing either the serosa (Lembert 1826), the serosa together with the mucosal surface (Czerny 1881) or the submucosa (Halstead 1887), modern techniques for creating an anastomosis such as stapler devices make no distinction and involve all these layers ${ }^{27}$.

Evidence demonstrating any superiority of stapled over hand-sewn techniques in colorectal anastomosis surgery is lacking, regardless of the level of anastomosis ${ }^{28}$. Besides suture methods, the configuration of the suture bite may be of interest. Both full-thickness and sero-submucosal sutures seem to be sufficient to anatomically apposition both sides of the bowel, thereby promoting wound healing ${ }^{29}$. However, studies reporting on suture bites are rare and could be of particular interest. It can be hypothesized that the submucosa of the bowel is of great importance in anastomotic healing since it is a though fibrous layer consisting mainly of collagen and elastin fibers and has the greatest tensile strength of the four layers ${ }^{30}$. Indeed, the submucosal layer is the source of fibroblasts that are activated after gastrointestinal surgery and start to deposit collagen. In an experimental model Daams and colleagues showed that healing of an everting anastomosis occurred by formation of a fibrotic cap at the serosal side, functioning as a matrix for fibroblasts ${ }^{31}$. This is in accordance with the first stage of classic wound repair: inflammation. Here, a fibrin matrix is formed as part of haemostasis, which serves as a scaffold for infiltrating cells ${ }^{32}$. However, the role of the mucosa is completely neglected in anastomotic healing when the submucosa is considered the most important layer.

In the early 90s, it has been demonstrated that an anastomosis causes a deep and long lasting reduction in energy metabolism, especially in the mucosa and the muscle layers ${ }^{33}$. The function of the mucosa in anastomotic healing and leakage should not be disregarded and may even play a more important role than currently recognized. Partly because bacteria house in the mucus of the colon and these bacteria are proposed as one of the causal factors in AL, which occurs when the healing process is disrupted ${ }^{34,35}$. In addition, our data showed that Muc2 knockout mice are more prone to the development of AL than control mice, indicating that a normal functioning mucus layer is essential for anastomotic healing (see also Chapter 5). Furthermore, macrophages in the gastrointestinal mucosa represent the largest pool of tissue macrophages in the body and a long-lasting macrophage 
absence or dysfunction impairs anastomotic healing36,37. Macrophages are one of the main factors in the inflammatory response, and based on their behaviour, this response is either pro-inflammatory (M1) impairing wound healing or antiinflammatory (M2) promoting wound healing; a shift in the M2/M1 index can influence the outcome of anastomotic healing ${ }^{38}$. The interaction between intraluminal content and the distinct layers of the bowel wall with their separate cell types and function, may be key in unravelling the healing process.

In summary, all layers of the bowel wall seem to have a role in anastomotic healing. The submucosa consists of connective tissue and has the greatest tensile strength of the four layers. Moreover, the serosa seems to be important in providing a matrix for fibroblasts, while the interaction between bacteria, mucus and the mucosal layer also seem important to maintain homeostasis in which anastomotic healing can occur. The focus of research into anastomotic healing should lie on transmural evaluation of the healing process and interaction between layers of the bowel wall, which was the aim of Chapter 3 of this thesis.

\section{Gastrointestinal healing versus cutaneous healing}

Although it is often believed that all tissue types heal in a similar fashion, this is not entirely true. Already in 1997, it was stated by Thornton that unlike cutaneous healing, healing of the gastrointestinal tract - more specifically the intestinal anastomosis - is anatomically obscured from inspection, allowing the surgeon to judge the success of the operation only on the patient's parameters of general wellbeing ${ }^{39}$. Not only is this bothersome in daily practice, it may also be part of the explanation that knowledge of gastrointestinal healing is lagging behind compared to skin wound healing.

The classic phases of wound healing (inflammation, proliferation and remodelling) have been studied extensively in skin ${ }^{40}$ and many researchers describe gastrointestinal healing in terms of these phases ${ }^{29,41}$. It is true that these 3 phases exist in all types of tissue, however, there are significant differences between skin and gastrointestinal healing ${ }^{42}$. These differences relate to factors such as collagen type, collagenase activity, wound strength and wound environment.

First of all, collagen subtypes in the gastrointestinal tract (I, III, V) are produced by fibroblasts and smooth muscle cells compared to the skin where solely type I and III are produced by fibroblasts. Collagenase activity plays an important role in the healing of anastomoses, where a high activity causes collagen lysis that results in low anastomotic strength early after the formation of an anastomosis ${ }^{43}$. Wound healing is far more rapid in the gastrointestinal tract than in the skin despite the potential risks such as shear stress, bacteria possibly affecting anastomotic healing 
and changes in vascular perfusion that are more abundant in the intestinal environment ${ }^{39,43}$.

The serosal layer plays an important role in terms of strength of the wound while there is no equivalent component in cutaneous healing. Not only are the components not similar, the reaction of both tissues also differs. For example, Törkvist and colleagues tried to block CD18-dependent neutrophil infiltration to improve wound healing and concluded that neutrophils may influence the wound healing process differently in specific organs, based on diverse results in skin and intestinal tract ${ }^{44}$. One of the explanations for their different results may lie in differences between cutaneous and intestinal collagen synthesis ${ }^{45}$, however, also the skin flora and gut microbiota vary completely, which can play an important role in differences in wound healing 46,47 .

In conclusion, gastrointestinal - and more specifically anastomotic - healing differs significantly from cutaneous healing. Although there are similarities, especially in the phases of wound healing, one cannot directly compare these two processes and therefore, gastrointestinal anastomotic healing should be considered a separate entity that needs to be investigated in more detail.

\section{The involvement of bacteria in the pathogenesis of anastomotic leakage}

From experimental studies, evidence suggests a positive effect of antibiotics on the strength of colonic anastomosis ${ }^{48,49}$. This implicates that when certain bacteria are being inhibited, this actually enhances anastomotic healing. Alverdy and colleagues have been investigating this hypothesis for some time and proposed a significant role for bacteria in the pathophysiology of AL in $2013^{50}$. Over the past few years, they have shown that virulent bacteria with high collagenase activity may contribute to the development of $\mathrm{AL}^{34}$. The interaction between intraluminal content and the several layers of the bowel wall may be key in the physiology of anastomotic healing. Since bacteria reside in the colonic mucus layer, they could be the reason why mice without normal mucus composition suffered more from AL, as stated previously. In addition, it has been shown that butyrate, a short-chain fatty acid (SCFA) produced by microbiota can strengthen colonic anastomoses in rats ${ }^{51}$. Already in 1973, it was demonstrated by Levison that SCFAs inhibited in vitro growth of P. Aeruginosa ${ }^{52}$ exactly the pathogen that was later identified as being able to transform into tissue destroying phenotype with high collagenase activity ${ }^{35}$. It seems undeniable that bacteria play a role in the pathogenesis of AL. However, clinical implications for these findings are lacking. It remains to be elucidated whether eliminating bacteria by perioperative antibiotics or promoting the growth of certain species with probiotics can improve anastomotic healing. Eliminating 
bacteria and faeces from the colon prior to surgery can be achieved with mechanical bowel preparation. Nevertheless, mechanical bowel preparation - that was traditionally used together with oral antibiotics - did not show any beneficial effect according to several randomized trials and was therefore abandoned. Nonetheless, a recent study attempted to clarify the effect of mechanical bowel preparation with or without antibiotics ${ }^{53}$, using a large retrospective cohort. Since the use of oral antibiotics alone has not been investigated in the majority of these studies, there is not enough evidence to conclude anything regarding oral antibiotics independently of mechanical bowel preparation.

In summary, given the available data, there seems to be a role for bacteria in the pathogenesis of anastomotic leakage. More research is needed to completely elucidate this role and the interaction of microbiota with specific cells and components at the anastomotic site.

\section{Surrogate markers of anastomotic healing}

The most frequently used surrogate marker for anastomotic healing in animal models is bursting pressure (BP). Although there are many methods to test BP, it all comes down to inflating or filling the bowel segment including the anastomotic site and measuring the intraluminal pressure at which either air or fluid leakage is observed at the site of the anastomosis. While this outcome measure has been debated for possibly disrupting tissue samples, making histological evaluation difficult and though critics have stated that BP is not a relevant indicator since it can not be applied for a anastomosis that has already leaked ${ }^{54}$; it is still considered appropriate by researchers in the field since it offers an actual surrogate outcome: anastomotic strength (see also Chapter 2). Quantitative comparison of BP is not valid between studies, since the protocols and instruments vary tremendously; therefore, only BP values can be directly compared between experimental groups in the same study 55 . Other mechanical tests such as measurement breaking strength or tensile strength are extensively criticized for either being not sensitive enough to measure in the early healing phase and because of the technical difficult implementation ${ }^{57}$.

Histological healing parameters are often reported in experimental studies investigating gastrointestinal anastomoses, mostly by grading scales including factors such as inflammatory cell infiltration, fibroblast activity, collagen deposition and vascularity or neoangiogenesis, factors that are mainly based on cutaneous wound healing 29,56-58. These parameters are considered helpful in evaluating the general wound healing process at the anastomotic site. However, a limitation may be that to investigate the true leak in the anastomotic line, histological evaluation has to be performed right at the spot of the leak. 
In wound healing, fibroblasts replace the provisional matrix that is established during inflammation with collagen-rich granulation tissue making collagen an interesting marker for anastomotic healing ${ }^{30}$. Therefore, other potential surrogate markers are analyses that measure collagen content, synthesis and degradation ${ }^{59}$. Quantification of collagen is often performed by measuring hydroxyproline content, since this amino acid is found in few proteins other than collagen ${ }^{22}$. Although hydroxyproline content is considered informative about the amount of collagen, it does not provide information on collagen subtypes, the maturity of the collagen and therefore not necessarily the tissue strength, since this lies more in the type and quality of collagen present in the anastomotic tissue ${ }^{60-62}$. Picro Sirius Red staining combined with digital imaging to analyse the percentage of collagen type I and III can demonstrate the ratio between young and mature collagen ${ }^{51,63}$. A disadvantage of this technique - and all immunohistochemistry analyses - is that it is a nonquantitative method, nevertheless it does locate the specific site in the tissue where the deposition of collagen occurs and current computer imaging techniques can aid in the quantification of different subtypes of collagen ${ }^{64}$. Collagen degradation can be mediated through Matrix Metalloproteinases (MMP) resulting in loosening of the matrix that may result in AL ${ }^{65}$. This collagenase activity of MMPs - especially MMP9 is associated with AL - can be measured by quantitative gelatin zymography $34,51,66,67$. Again the statement of the spatial and regional context of measuring bacteria and inflammatory mediators applies here; in grinding up tissues you are getting the average of the entire tissue sample, while the most important measure is likely to be right at the site of the necrosis and leak. However, it has been demonstrated that it is possible to distinguish between changes in the composition of the intestinal microbiota associated with anastomotic tissue and microbiota associated with luminal contents ${ }^{68}$.

In summary, frequently used surrogate markers for anastomotic healing in animal models are bursting pressure, tensile strength and a generic histological examination. Other additional analyses are used to answer specific research question, but new techniques are necessary to obtain more insight in the anastomotic healing process.

\section{Aim and outline of this thesis}

Although this thesis does not provide indisputable answers to the discussion points described above, it does provides new insights into research on anastomotic leakage. This thesis is divided into four parts and describes discernments gained from animal studies regarding bowel anastomoses in the lower gastrointestinal tract. 
In Part I, the use of animal models for anastomotic healing in the lower gastrointestinal tract is investigated. A systematic review of the literature is given providing an overview of recent literature combined with recommendations from a recent Delphi analysis (Chapter 2). These recommendations enable future researchers to perform their research in such a way that it is more comparable with other animal studies and more translatable to the human setting. Furthermore, new imaging techniques make it possible to focus more in depth on the process of anastomotic healing using mass-spectrometry-imaging (Chapter 3) reducing the amount of animals needed to obtain a broad range of information.

Part II focuses on the identification of risk factors for anastomotic leakage. It is known from clinical practice that the perioperative use of non-steroidal antiinflammatory drugs increases the risk of developing anastomotic leakage, possibly due to the inhibition of Cyclooxygenase-2 (COX-2). The role of COX-2 in colorectal anastomotic healing (Chapter 4) as well as the necessity of normal mucus composition (Chapter 5) is investigated using knockout mice model.

The aim of Part III is to reduce anastomotic leakage rate in animal models using several different interventions. The positive effect of butyrate on the strength of colorectal anastomosis was already known, so new approaches - which are described in Chapter $\mathbf{6}$ - are necessary to make the translation into the human setting. In Chapter 7, the use of glues to reduce anastomotic leakage in a rat model was investigated in collaboration with colleagues from the Erasmus Medical Center in Rotterdam.

In Part IV the balance between anastomotic healing and adhesion formation is investigated. With new approaches to prevent adhesions after abdominal surgery, there may be a potential risk of disrupting the normal anastomotic healing process. Therefore, it is important to investigate the effect of such anti-adhesion barriers on colorectal anastomoses in animals (Chapter 8). Also the use of new biomaterials can have a negative effect on the abdominal environment that may cause a disruption in normal healing processes (Chapter 9).

Chapter 10 summarizes the main results and contributions as presented in this thesis; it depicts future perspectives and implications for further experimental research and clinical applications. 


\section{References}

1. Ferlay J, Steliarova-Foucher E, Lortet-Tieulent J, Rosso S, Coebergh JWW, Comber H, et al. Cancer incidence and mortality patterns in Europe: estimates for 40 countries in 2012. Eur J Cancer 2013;49(6):1374-1403.

2. Volksgezondheidenzorg.info (2015) website, RIVM: Bilthoven

3. Jaarrapportage 2012: uitkomst van zorg registratie. Dutch Surgical Colorectal Audit, retrieved from: www.clinicalaudit.nl/dsca

4. McArdle CS, McMillan DC, Hole DJ. Impact of anastomotic leakage on long-term survival of patients undergoing curative resection for colorectal cancer. BJS. 2005;92(9):1150-1154.

5. Khan AA, Wheeler JMD, Cunningham C, George B, Kettlewell M, Mortensen NJM. The management and outcome of anastomotic leaks in colorectal surgery. Colorectal Disease, 2008;10(6):587-592.

6. Walker KG, Bell SW, Rickard MJFX, Mehanna D, Dent OF, Chapuis PH, Bokey EL. Anastomotic leakage is predictive of diminished survival after potentially curative resection for colorectal cancer. Ann Surg, 2004;240(2):255-259.

7. Mirnezami A, Mirnezami R, Chandrakumaran K, Sasapu K, Sagar P, Finan P. Increased local recurrence and reduced survival from colorectal cancer following anastomotic leak. Ann of Surg, 2011;253(5):890-899.

8. Krarup P-M, Nordholm-Carstensen A, Jorgensen LN, Harling H. Anastomotic leak increases distant recurrence and long-term mortality after curative resection for colonic cancer. Ann of Surg, 2014;259(5):930-938.

9. NC3Rs Reporting Guidelines Working Group. Animal research: reporting in vivo experiments: the ARRIVE guidelines. J Physiol. 2010;588(Pt 14):2519-2521

10. Teixeira da Silva JA. Negative results: negative perceptions limit their potential for increasing reproducibility. J Negat Results Biomed. 2015;14:12

11. Morks AN, Havenga K, Ploeg RJ. Can intraluminal devices prevent or reduce colorectal anastomotic leakage: a review. World J Gastroenterol. 2011;17(40):4461-4469.

12. Matsuda M, Tsuruta M, Hasegawa H, Okabayashi K, Kondo T, Shimada T, Yahagi M, Yoshikawa Y, Kitagawa Y. Transanal drainage tube placement to prevent anastomotic leakage following colorectal cancer surgery with double stapling reconstruction. Surg Today. 2016;46(5):613-620..

13. Nachiappan S, Datta U, Askari A, Faiz O. Tube ileostomy as a faecal diversion for elective distal colorectal anastomoses: a systematic review and pooled analysis. Colorectal Dis. 2015;17:665-673

14. Hidaka E, Ishida F, Mukai S, Nakahara K, Takayanagi D, Maeda C, Takehara Y, Tanaka J, Kudo SE. Efficacy of transanal tube for prevention of anastomotic leakage following laparoscopic low anterior resection for rectal cancers: a retrospective cohort study in a single institution. Surg Endosc. 2015;29(4):863-867.

15. Bakker IS, Morks AN, Ten Cate Hoedemaker HO, Burgerhof JGM, Leuvenink HGD, Ploeg RJ, Havenga K. Interim analyse van de C-seal trial. Chirurgendagen 2014. Retrieved from: http://cd2014.chirurgendagen.nl/programma/sessie/3157/57

16. Nordentoft T, Pommergaard HC, Rosenberg, J, Achiam MP. Fibrin Glue Does Not Improve Healing of Gastrointestinal Anastomoses: A Systematic Review. Eur Surg Res. 2015;54(1-2):1-13.

17. Vakalopoulos KA, Wu Z, Kroese L, Kleinrensink GJ, Jeekel J, Vendamme R, Dodou D, Lange JF. The prevention of colorectal anastomotic leakage with tissue adhesives in a contaminated environment is associated with the presence of anti-inflammatory macrophages. Ann Surg. 2015;261(2):323-331

18. Wu Z, Vakalopoulos KA, Boersema GS, Kroese LF, Lam KH, van der Horst PH, Mulder IM, Bastiaansen-Jenniskens YM, Kleinrensink GJ, Jeekel J, Lange JF. The prevention of colorectal anastomotic leakage with tissue adhesives in a contaminated environment is associated with the presence of anti-inflammatory macrophages.Int J Colorectal Dis. 2014;29(12):1507-1516.

19. Ríos Castellanos E, Seron P, Gisbert JP, Bonfill Cosp X. Endoscopic injection of cyanoacrylate glue versus other endoscopic procedures for acute bleeding gastric varices in people with portal hypertension. Cochrane Database Syst Rev. 2015 May 12;5:CD010180 
20. Stam MAW, Mulder CLJ, Consten ECJ, Tuynman JB, Buskens CJ, Bemelman WA. Sylys® surgical sealant: a safe adjunct to standard bowel anastomosis closure. Ann Surg Innov Rese 2014;8:

21. Vakalopoulos KA, Daams F, Wu Z, Timmermans L, Jeekel JJ, Kleinrensink GJ, van der Ham A, Lange JF. Tissue adhesives in gastrointestinal anastomosis: a systematic review. J Surg Res. 2013;180(2):290-300.

22. Hendriks T, Mastboom WJB. Healing of experimental intestinal anastomoses: parameters for repair. Dis Colon Rectum. 1990; 33:891-901.

23. Ten Broek RP, Issa Y, van Santbrink EJ, Bouvy ND, Kruitwagen RF, Jeekel J, Bakkum EA, Rovers MM, van Goor H. Burden of adhesions in abdominal and pelvic surgery: systematic review and met-analysis. BMJ. 2013 3;347:f5588.

24. ten Broek RP, Stommel MW, Strik C, van Laarhoven CJ, Keus F, van Goor H. Benefits and harms of adhesion barriers for abdominal surgery: a systematic review and meta-analysis. Lancet. 2014;383(9911):48-59.

25. Slieker JC, Ditzel M, Harlaar JJ, Mulder IM, Deerenberg EB, Bastiaansen-Jenniskens YM, Kleinrensink GJ, Jeekel J, Lange JF. Effects of new anti-adhesion polyvinyl alcohol gel on healing of colon anastomoses in rats. Surg Infect (Larchmt). 2012;13(6):396-400.

26. Shogan BD, An GC, Schardey HM, Matthews JB, Umanskiy K, Fleshman JW, et al. Proceedings of the first international summit on intestinal anastomotic leak, Chicago, Illinois, October 4-5, 2012. Surg Infect (Larchmt). 2014:479-489.

27. Slieker, J.C. (2014, February 26). Improving Quality in Colorectal Surgery. Erasmus University Rotterdam. Retrieved from: http://hdl.handle.net/1765/50545

28. Neutzling CB, Lustosa SAS, Proenca IM, da Silva EMK, Matos D. Stapled versus handsewn methods for colorectal anastomosis surgery. Matos D, editor. Cochrane Database Syst Rev. 2012;2: CD003144.

29. Thompson SK, Chang EY, Jobe BA. Clinical review: Healing in gastrointestinal anastomoses, part I. Microsurgery. 2006;26(3):131-136.

30. Slieker JC, Daams F, Mulder IM, Jeekel J, Lange JF. Systematic review of the technique of colorectal anastomosis. JAMA Surg. 2013;148(2):190-201.

31. Daams F, Monkhorst K, van den Broek J, Slieker JC, Jeekel J, Lange JF. Local Ischaemia Does Not Influence Anastomotic Healing: An Experimental Study. Eur Surg Res. 2013;50(1):24-31.

32. Gurtner GC, Werner S, Barrandon Y, Longaker MT. Wound repair and regeneration. Nature. 2008; 453(7193):314-321.

33. Braskén P. Healing of experimental colon anastomosis. Eur J Surg Suppl. 1991;(566):1-51.

34. Shogan BD, Belogortseva N, Luong PM, Zaborin A, Lax S, Bethel C, et al. Collagen degradation and MMP9 activation by Enterococcus faecalis contribute to intestinal anastomotic leak. Sci Transl Med. 2015;7(286):286ra68-8.

35. Olivas AD, Shogan BD, Valuckaite V, Zaborin A, Belogortseva N, Musch M, et al. Intestinal tissues induce an SNP mutation in Pseudomonas aeruginosa that enhances its virulence: possible role in anastomotic leak. PLoS One. 2012;7(8):e44326.

36. Pantelis D, Beissel A, Kahl P, Vilz TO, Stoffels B, Wehner S, et al. Colonic anastomotic healing in the context of altered macrophage function and endotoxemia. Int J Colorectal Dis. 2011;26:737-746.

37. Smith PD, Smythies LE, Shen R, Greenwell-Wild T, Gliozzi M, Wahl SM. Intestinal macrophages and response to microbial encroachment. Mucosal Immunol. 2011;4(1):31-42.

38. Wu Z, Vakalopoulos KA, Boersema GSA, Kroese LF, Lam KH, van der Horst PH, et al. The prevention of colorectal anastomotic leakage with tissue adhesives in a contaminated environment is associated with the presence of anti-inflammatory macrophages. Int J Colorectal Dis. 2014;29(12):1507-1516.

39. Thornton FJ, Barbul A. Healing in the gastrointestinal tract. Surg Clin North Am. 1997;77:549-573.

40. Gurtner GC, Werner S, Barrandon Y, Longaker MT. Wound repair and regeneration. Nature. 2008;453(7193):314-321.

41. van der Vijver RJ, van Laarhoven CJHM, de Man BM, Lomme RMLM, Hendriks T. The effect of fibrin glue on the early healing phase of intestinal anastomoses in the rat. Int J Colorectal Dis. 2012;27(8):1101-1107. 
42. Hoeppner J, Wassmuth B, Marjanovic G, Timme S, Hopt UT, Keck T. Anastomotic sealing by extracellular matrices (ECM) improves healing of colonic anastomoses in the critical early phase. J Gastrointest Surg. 2010;14(6):977-986.

43. Munireddy S, Kavalukas SL, Barbul A. Intra-abdominal healing: gastrointestinal tract and adhesions. Surg Clin North Am. 2010;90(6):1227-1236.

44. Törkvist L, Månsson P, Raud J, Larsson J, Thorlacius H. Role of CD18-dependent neutrophil recruitment in skin and intestinal wound healing. Eur Surg Res. 2001;33(4):249-254.

45. Martens MF, Huyben CM, Hendriks TH. Collagen synthesis in fibroblasts from human colon: regulatory aspects and differences with skin fibroblasts. Gut. 1992;33(12):1664-1670.

46. Grice EA, Segre JA. The skin microbiome. Nat Rev Microbiol. 2011;9(4):244-253.

47. Jandhyala SM, Talukdar R, Subramanyam C, Vuyyuru H, Sasikala M, Nageshwar Reddy D. Role of the normal gut microbiota. World J Gastroenterol. 2015;21(29):8787-8803.

48. Binnebösel M, Junge K, Kaemmer DA, Krones CJ, Titkova S, Anurov M, et al. Intraperitoneally applied gentamicin increases collagen content and mechanical stability of colon anastomosis in rats. Int J Colorectal Dis. 2009;24(4):433-440.

49. Pasternak B, Rehn M, Andersen L, Ågren MS, Heegaard A-M, Tengvall P, et al. Doxycycline-coated sutures improve mechanical strength of intestinal anastomoses. Int $\mathrm{J}$ Colorectal Dis. 2007;23(3):271-276.

50. Shogan BD, Carlisle EM, Alverdy JC, Umanskiy K. Do we really know why colorectal anastomoses leak? J Gastrointest Surg. 2013;17(9):1698-1707.

51. Bloemen JG, Schreinemacher MH, de Bruine AP, Buurman WA, Bouvy ND, Dejong CH. Butyrate enemas improve intestinal anastomotic strength in a rat model. Dis Colon Rectum. 2010; 53(7):1069-1075.

52. Levison ME. Effect of colon flora and short-chain fatty acids on growth in vitro of Pseudomonas aeruginsoa and Enterobacteriaceae. Infect Immun. 1973;8(1):30-35.

53. Kiran RP, Murray ACA, Chiuzan C, Estrada D, Forde K. Combined preoperative mechanical bowel preparation with oral antibiotics significantly reduces surgical site infection, anastomotic leak, and ileus after colorectal surgery. Ann of Surg. 2015;262(3):416-425-discussion423-425.

54. Rushfeldt CF, Sveinbjørnsson B, Søreide K, Vonen B. Risk of anastomotic leakage with use of NSAIDs after gastrointestinal surgery. Int J Colorectal Dis. 2011;26(12):1501-1509.

55. Månsson P, Zhang XW, Jeppsson B, Thorlacius H. Anastomotic healing in the rat colon: comparison between a radiological method, breaking strength and bursting pressure. Int J Colorectal Dis. 2002;17(6):420-425.

56. Hiradfar M, Shojaeian R, Zabolinejad N, Gharavifard M, Sabzevari A, Joodi M, et al. "Tie over ring" sutureless compression based gastrointestinal anastomotic method: experimental rat model. J Pediatr Surg. 2014;49(3):405-409.

57. Hirai K, Tabata Y, Hasegawa S, Sakai Y. Enhanced intestinal anastomotic healing with gelatin hydrogel incorporating basic fibroblast growth factor. J Tissue Eng Regen Med. 2013 Oct 16.

58. Fresno L, Fondevila D, Bambo 0, Chacaltana A, García F, Andaluz A. Effects of platelet-rich plasma on intestinal wound healing in pigs. Vet J. 2010;185(3):322-327.

59. Martens MF, Hendriks T. Postoperative changes in collagen synthesis in intestinal anastomoses of the rat: differences between small and large bowel. Gut. 1991;32(12):1482-1487.

60. Klein M. Postoperative non-steroidal anti-inflammatory drugs and colorectal anastomotic leakage. NSAIDs and anastomotic leakage. Dan Med J. 2012;59(3):B4420-0.

61. de Hingh IHJT, van Goor H, de Man BM, Lomme RMLM, Bleichrodt RP, Hendriks T. Selective cyclooxygenase 2 inhibition affects ileal but not colonic anastomotic healing in the early postoperative period. Br J Surg. 2006;93(4):489-497.

62. da Costa MAR, Campos ACL, Coelho JCU, de Barros AM, Matsumoto HM. Oral glutamine and the healing of colonic anastomoses in rats. JPEN J Parenter Enteral Nutr. 2003;27(3):182-185, discussion185-186.

63. Binnebösel M, Grommes J, Koenen B, Junge K, Klink CD, Stumpf M, et al. Zinc deficiency impairs wound healing of colon anastomosis in rats. Int J Colorectal Dis. 2009;25(2):251-257. 
64. Sánchez-De Pedro F, Moreno-Sanz C, Morandeira-Rivas A, Tenías-Burillo JM, Alhambra-Rodríguez De Guzmán C. Colorectal anastomosis facilitated by the use of the LigaSure ${ }^{\circledR}$ sealing device: comparative study in an animal model. Surg Endosc. 2013;28(2):508-514.

65. Stumpf M, Klinge U, Wilms A, Zabrocki R, Rosch R, Junge K, et al. Changes of the extracellular matrix as a risk factor for anastomotic leakage after large bowel surgery. Surgery. 2005; 137(2):229-234.

66. de Hingh IHJT, de Man BM, Lomme RMLM, van Goor H, Hendriks T. Colonic anastomotic strength and matrix metalloproteinase activity in an experimental model of bacterial peritonitis. Br J Surg. 2003;90(8):981-988.

67. van der Vijver RJ, van Laarhoven CJHM, de Man BM, Lomme RMLM, Hendriks T. Perioperative pain relief by a COX-2 inhibitor affects ileal repair and provides a model for anastomotic leakage in the intestine. Surg Innov. 2013;20(2):113-118.

68. Shogan BD, Smith DP, Christley S, Gilbert JA, Zaborina O, Alverdy JC. Intestinal anastomotic injury alters spatially defined microbiome composition and function. Microbiome. 2014;2(1):35. 


\section{Part I}

Animal models for research on anastomotic healing 


\section{CHAPTER 2}

\section{International consensus statement regarding the use of animal models for research on anastomoses in the lower gastrointestinal tract}




\section{Abstract}

\section{Purpose}

This project aimed to reach consensus on the most appropriate animal models and outcome measures in research on anastomoses in the lower gastrointestinal tract (GIT). The physiology of anastomotic healing remains an important research topic in gastrointestinal surgery. Recent results from experimental studies are limited with regard to comparability and clinical translation.

\section{Methods}

PubMed and EMBASE were searched for experimental studies investigating anastomotic healing in the lower GIT published between January 1, 2000 - December 31, 2014 to assess currently used models. All corresponding authors were invited for a Delphi-based analysis that consisted of 2 online survey rounds followed by a final online recommendation survey to reach consensus on the discussed topics.

\section{Results}

277 original articles were retrieved and 167 articles were included in the systematic review. Mice, rats, rabbits, pigs and dogs are currently being used as animal models, with a large variety in surgical techniques and outcome measures.

Forty-four corresponding authors participated in the Delphi analysis. In the first 2 rounds 39/44 and 35/39 participants completed the survey. In the final meeting 35 experts reached consensus on 76/122 items in 6 categories. Mouse, rat and pig are considered appropriate animal models; rabbit and dog should be abandoned in research regarding bowel anastomoses. ARRIVE guidelines should be followed more strictly.

\section{Conclusions}

Consensus was reached on several recommendations for the use of animal models and outcome measurements in research on anastomoses of the lower GIT. Future research should take these suggestions into account to facilitate comparison and clinical translation of results. 


\section{Introduction}

Anastomotic leakage (AL) is one of the most dreaded complications after colorectal surgery and leads to high morbidity and mortality ${ }^{1,2}$. Despite accumulated knowledge, improvement of surgical techniques and ongoing research on this topic, the incidence of colorectal AL remains approximately $11 \%$ worldwide 3 .

Animal models are used on a regular basis to investigate normal healing of an intestinal anastomosis as well as leakage of the anastomosis. These animal models are vital for our understanding of anastomotic healing and introduction of new therapies for reduction of AL. However, over the past decades a variety of animal models have been used which leads to heterogeneity, accompanied by differing anatomy and physiology between species. Recently, a systematic review concluded that animal research on AL is of poor quality and improvement is needed before results can be translated into the human setting 4 .

In addition to the variety of animal models, a wide range of study endpoints and/or goals is used. The majority of studies examined the effect of a certain intervention on anastomotic healing, for example aiming at improving anastomotic strength or reducing leakage rate in models of insufficient anastomoses. Several studies have focused on different techniques to perform the anastomosis, for example evaluating or enforcing suturing techniques or various types of staplers $^{5-10}$. Glues and patches have also been used to cover the anastomosis in an attempt to decrease leakage rate ${ }^{11-19}$. Other methods include stenting the lumen of the intestine or providing specific medication to improve wound healing $20-28$. The influence of specific drugs that may attenuate the anastomotic healing process was also investigated $29-32$. Due to the large variance in study design, outcome measures and analyses for surrogate markers of anastomotic healing, the question arises whether there is one specific animal model suitable to investigate all these different research topics. Furthermore, the role of limitations such as availability, costs, handling and housing requirements in choosing an animal model remains unclear and may also influence why certain animal models are currently being used.

To date, a single recommendation on the use of animal models for clinical colon AL has been proposed by Pommergaard et al in 201133. Based on a systematic review the authors first listed pros and cons for different experimental animals and subsequently opted for the mouse as best suited to mimic clinical colon AL. However, despite this recommendation, the use of mice to study bowel anastomoses has not been universally adopted. This suggests that there is either insufficient knowledge or limited support from researchers in the field. This lack of 
consensus, and the resulting inconsistencies and differences between reported research limit transparency and opportunities to compare results between studies ${ }^{4}$.

Ideally, the most suited model can be selected based on clear evidence regarding reproducibility and validity of a model. However, in animal research regarding bowel anastomoses there is no single animal model that is evidently the most appropriate regarding practical ease, costs, reproducibility and clinical translation. Therefore, expert consensus is a suitable method to achieve homogeneity in the selection of animal models. If consensus can be reached, there will be more support from fellow researchers leading to more frequent use of similar models. As a consequence, future research about anastomotic healing will become more comparable.

The aim of this study is to review different experimental studies in which an animal model was used to investigate either intestinal anastomotic healing or leakage and obtain information on the used animal models; location \& type of surgery; macroscopic outcome; histological assessment; mechanical \& biochemical outcome measures and animal testing \& welfare. Further, we aim to reach consensus on these subjects by performing a Delphi-based analysis using an online survey to collect judgements of animal surgeons who performed the studies reviewed here.

\section{Methods}

\section{Literature search and study selection}

In December 2014, an extensive literature search was conducted using the PubMed (MEDLINE) and OvidSP (EMBASE) databases for all papers related to animal models which were used in an experimental setting to either investigate anastomotic healing or anastomotic leakage in the lower gastrointestinal tract. Search terms included: "anastomosis/anastomotic", "leak/leakage", "bowel/ intestine/colon/colorectal" and "animal/animals/rat/mouse/mice/pig/dog/goat/ rabbit/animal model". English and Dutch were used as language restrictions and the search was limited to articles published between January 1, 2000 and December 1, 2014. The following inclusion criteria were applied to the titles and abstracts of the search results: experimental setting, use of an animal model and an anastomosis made in the lower gastrointestinal tract (GIT) (gastroduodenal/ gastrojejunal were considered upper GIT and therefore excluded). We excluded commentary reports, review articles and articles containing results that had been previously reported in another included article. All articles were combined in a 
single list of which JWAMB and LvB identified eligible reports; in case of discrepancy, agreement on inclusion was reached through discussion with MA-T as a third reviewer. For an overview of the study selection, performed according to the Preferred Reporting Items for Systematic Reviews and Meta-Analyses (PRISMA) guideline, see Figure 2.1.

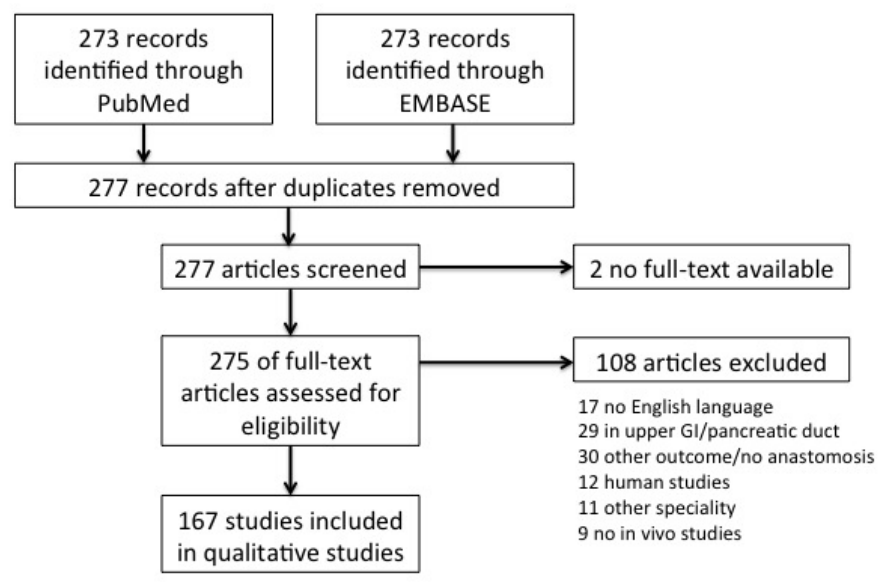

Figure 2.1 Prisma flow chart of the review process.

\section{Study outcomes}

Our primary outcomes were 1) type of animal used and 2) location and type of anastomosis. As secondary outcomes, we evaluated scoring models used for macroscopic findings reporting on AL, i.e. adhesions, bursting pressure, histology and other examinations performed. Further, we assessed the country of origin where the study was performed and the year in which the study was published and how animal welfare was reported.

\section{Online survey - Adaptation from the Delphi technique}

The qualitative review of the literature served as our starting point for the Delphi technique. The main goal was to achieve consensus on the use of animal models for research on anastomoses in the lower gastrointestinal tract, specifically on which animal, location \& type of surgery; macroscopic outcome; histological assessment; mechanical \& biochemical outcome measures and animal testing \& welfare. The Delphi technique is a widely used and accepted consensus method for gathering data from respondents within their domain of expertise in order to formulate recommendations or guidelines that can be used in the future. For this report, we 
contacted all principal investigators from included articles by email, explained our study and invited them to participate in this international Delphi project.

Questionnaires were developed and distributed using SurveyMonkey (SurveyMonkey Inc, Palo Alto, CA; www.survey-monkey.com). This online survey contained several questions on three main subtopics of intestinal anastomotic research: the first part consisted of questions regarding animal model used and reasoning for the choice of this model; the second part focused on macroscopically scoring and measurements performed on the anastomosis (leakage rate, adhesion evaluation) and the last part inquired about histological analysis and additional tests (e.g. bursting pressure, ELISA, qPCR) that were used to gain more insight in the healing process (inflammation, proliferation). There were no open questions in the survey, but participants were encouraged to provide arguments for their choice, suggestions or additional remarks in free text fields below each question.

After receiving participants' responses, the collected information was converted into a second questionnaire. This round included the items and ratings summarized from the previous round. Here we asked all participants to revise their judgments or specify reasons why they were not convinced of the most commonly used animal model. All items achieving consensus, remaining items and their ratings as well as minority opinions were reported during the questionnaires. The RAND/UCLA Appropriate Method (RAM) ${ }^{34}$ was used to assess consensus in an expert panel on the use of animal models, macroscopic scoring of leakage and adhesion, mechanical and biochemical parameters and histological outcome. For reporting our study, we used the recommendations of Sinha et al regarding the Delphi technique ${ }^{35}$.

\section{Statistical analysis}

Results of the survey were exported to MS Excel 2011 (Microsoft Corp, Redmond WA). Consensus was reached if the panel rated the subject inappropriate (panel median 1-3) or appropriate (panel median 7-9) on a 9-points scale without disagreement, according to the method used by Moossdorff et al. ${ }^{36}$. Disagreement was tested using the interpercentile range adjusted for symmetry in accordance to the RAND/UCLA Appropriateness Method Manual ${ }^{34}$

\section{Results}

\section{Literature findings}

In total 277 articles were retrieved from the search. After screening 167 articles were included in the systematic review (Figure 2.1). An on-going increase in 
publication frequency on anastomotic healing and leakage was found, from only 3 in 2000 to 18 in 2014 (Figure 2.2a). Animal models used in these experiments were rat (65\%), pig (15\%), rabbit (10\%), mouse (5\%) and dog (5\%) (Figure $2.2 \mathrm{~b})$. From all 167 studies, only 4 reported laparoscopic surgery in animals, 3 in pig models $s^{10,37,38}$ and one in a rat model ${ }^{39}$. One study performed transanal endoscopy ${ }^{40}$, while all other studies used laparotomy. Research was mainly performed in Europe, with several research groups responsible for a relatively large contribution to the total number of articles (Figure 2.2c).

Outcome measures reported in the included studies were: anastomotic healing, anastomotic leakage, bursting pressure, tensile strength, adhesion scores and histological parameters such as influx of granulocytes and collagen deposition. We summarized the characteristics of the used interventions and outcome measures that were used in these studies. This summary was sent to panel members as background information when completing the questionnaires (see supplemental data online: doi:10.1007/s00384-016-2550-5).
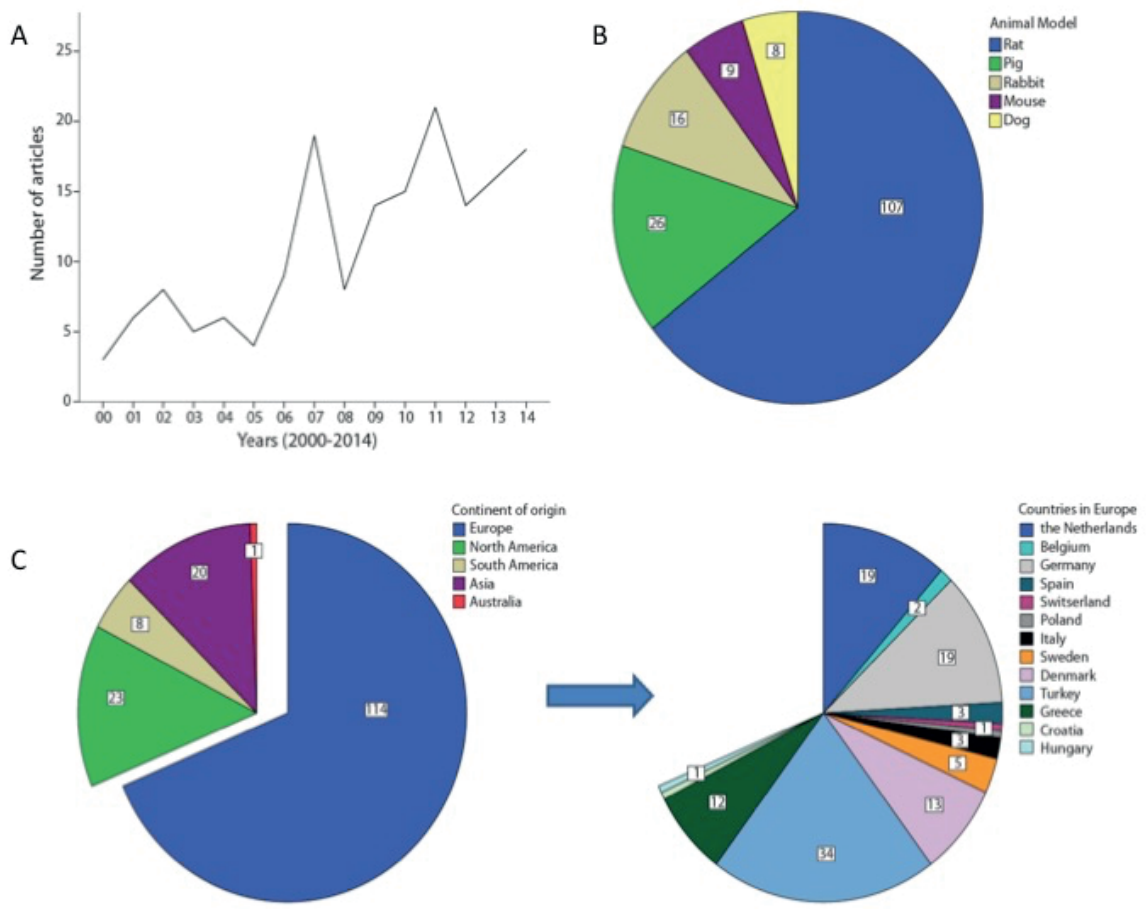

Figure 2.2 Incidence of articles published over the past 14 years (A). Animal models used in the studies (B) and origin of published articles (C). 


\section{Participation}

In total, 44 authors were willing to participate in the Delphi analysis, together being responsible for 77 of the included articles from the major research groups worldwide (Figure 2.3). The first questionnaire was completed by 39/44 responders (89\% response rate). After non-responders were excluded, the second round was completed by 35/39 panel members, all of whom also completed the final round (Table 2.1). No additional people were invited as the Delphi progressed.

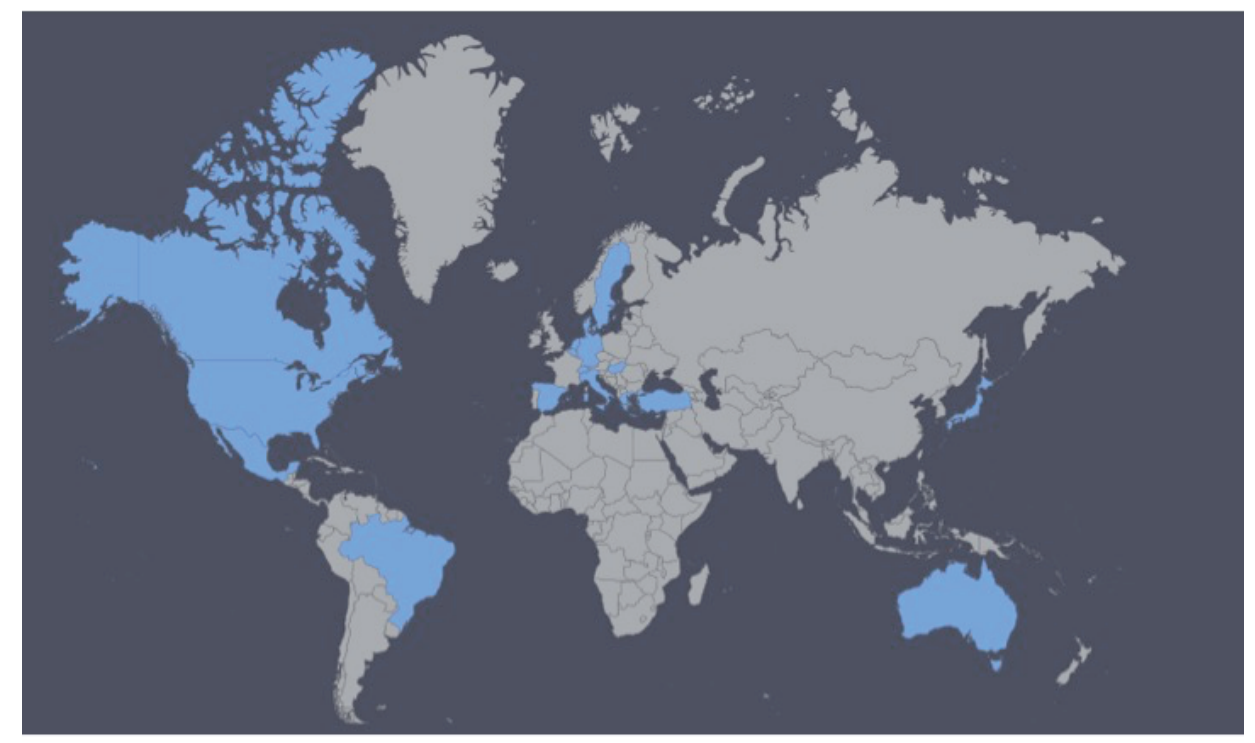

Figure 2.3 Participants in this Delphi analysis were from the main research groups that have published work on the subject of bowel anastomoses in animals, results obtained during the first survey (adapted from amcharts.com).

\section{Characteristics of panel members}

The occupation of the panel members is summarized in Table 2.2. Researchers had operated on approximately 200 animals and have published a median of 4 articles regarding this topic. All panel members were affiliated with a university and have experience performing animal research on this topic. The institutions listed in Table 2.1 were not involved in this project and do not necessarily subscribe to the consensus. 
Table 2.1 Panel members that completed the entire Delphi analysis.

\begin{tabular}{|c|c|}
\hline Panel members & Institute \\
\hline Magnus Ågren & University of Copenhagen, Denmark \\
\hline John Alverdy & University of Chicago Medical Center, Chicago, IL, USA \\
\hline Marcel Binnebösel & University Hospital of the RWTH, Aachen, Germany \\
\hline Wim Ceelen & Ghent University Hospital, Belgium \\
\hline Kadir Cetinkaya & Ankara Oncology Education and Research Hospital, Turkey \\
\hline Jorge Cueto & Anahuac University, Mexico \\
\hline Freek Daams & VU University Medical Center, The Netherlands \\
\hline Alyssa Fajardo & Washington University, St. Louis, MO, USA \\
\hline Laura Fresno & Autonomous University of Barcelona, Spain \\
\hline Wolfgang Gaertner & University of Minnesota, Minneapolis, MN, USA \\
\hline Torben Glatz & University Hospital Freiburg, Germany \\
\hline Jens Höppner & University Hospital Freiburg, Germany \\
\hline Niels Komen & University Hospital Leuven, Belgium \\
\hline Guy Maddern & University of Adelaide, Australia \\
\hline Antonio Morandeira-Rivas & "La Mancha Centro" General Hospital, Spain \\
\hline Tyge Nordentoft & University of Copenhagen, Denmark \\
\hline Adrian Park & $\begin{array}{l}\text { Johns Hopkins University School of } \\
\text { Medicine, Maryland, MD, USA }\end{array}$ \\
\hline Pablo Parra Membrives & Valme University Hospital, Seville, Spain \\
\hline Rogério Parra & University of São Paulo, Brazil \\
\hline Troy Perry & University Medical Center Alberta, Canada \\
\hline Hans-Christian Pommergaard & University of Copenhagen, Denmark \\
\hline Manousos-Georgios Pramateftakis & Aristotle University of Thessaloniki, Greece \\
\hline \multirow[t]{2}{*}{ Dimitrios Raptis } & Aristotle University of Thessaloniki, Greece \& \\
\hline & Friedrich-Alexander University of Erlangen, Germany \\
\hline Francisco Sánchez-De Pedro & "La Mancha Centro" General Hospital, Spain \\
\hline Marc Schreinemacher & Maastricht University Medical Center, The Netherlands \\
\hline Baddr Shakhsheer & University of Chicago Medical Center, Chicago, IL, USA \\
\hline Juliette Slieker & CHUV University Hospital Lausanne, Switzerland \\
\hline Lisette te Velde & VU University Medical Center, The Netherlands \\
\hline Hideo Terashima & University of Tsukuba, Japan \\
\hline Bobby Tingstedt & Lund University, Sweden \\
\hline Go van Dam & University Medical Center Groningen, The Netherlands \\
\hline Harry van Goor & Radboud University Medical Center, The Netherlands \\
\hline Zhouqiao Wu & Erasmus University Medical Center, The Netherlands \\
\hline Simon Yauw & Radboud University Medical Center, The Netherlands \\
\hline Oded Zmora & Sheba Medical Center, Tel Aviv, Israel \\
\hline
\end{tabular}

Table 2.2 Current functions of the panel members, results obtained during the first survey.

\begin{tabular}{lc}
\hline Function & $(\mathrm{N})$ \\
\hline PhD Candidate & 5 \\
MD/resident/surgeon & 9 \\
Postdoctoral researcher & 4 \\
Assistant Professor & 11 \\
Professor & 10 \\
\hline
\end{tabular}

All panel members have conducted hands-on animal experiments, mostly during their PhD-trajectory $(17 / 35)$. 


\section{First questionnaire}

The first questionnaire consisted of 95 items in 6 categories, namely the choice of animal model; location and technique of the anastomosis; macroscopic outcome; histological assessment; mechanical and biochemical outcome measures; and reporting specifics on animal research.

After the first round, consensus existed on 58 of the items (61\%) and disagreement or uncertainty on 37 items. Based on additional remarks, 7 items were added and 2 were rephrased. The 7 newly introduced items for the second round consisted of additional techniques in the category mechanical and biochemical outcome measures and a suggestion by one of the panel members for a new macroscopic scoring system.

\section{Second questionnaire}

The second questionnaire was based on the first and consisted of 37 items on which consensus did not exist in the first round and 7 items added based on additional comments (total of 44 items). The most important item that was added in round 2 and on which immediate consensus was reached was the newly introduced Anastomotic Complication Score (ACS, see Table 2.3), which was proposed during the first round by one of the panel members. After the second round, consensus existed only on 3 items, in addition to the 58 items on which consensus was reached in the first round.

Table 2.3 Anastomotic Complication Score for macroscopic outcome in animal research regarding bowel anastomoses.

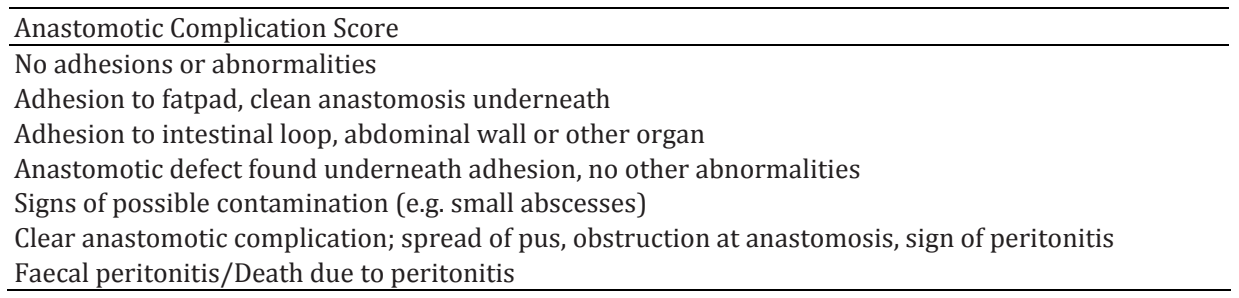

\section{Final round}

Feedback was provided to the participants after each round. In the final round a clear distinction was made between positive and negative arguments from the second round to simulate a discussion between panel members. The topics for debate remained why certain animal models should or should not be used. Some panel members argued that small animal models are not able to reflect the clinical setting while others are certain that with the right scoring systems one can obtain 
sufficient information to make the model more translational. Based on all arguments given by the panel members and the first two rounds, 20 recommendations were proposed. Consensus was reached on 17 of those recommendations (Tabel 2.4).

Table 2.4 Summary of the consensus on the use of animal models for bowel anastomoses in the lower gastrointestinal tract.

\begin{tabular}{|c|c|}
\hline Category & Consensus \\
\hline \multirow{5}{*}{$\begin{array}{l}\text { Selection of } \\
\text { animal model }\end{array}$} & - Mouse, rat and pig models are considered appropriate models. \\
\hline & - Choice of animal model depends on research question. \\
\hline & - A rat model is preferred to a mouse model (mostly because of size) however \\
\hline & knockout mice are helpful in answering specific research questions. \\
\hline & - Rabbit and dog models are not validated and are considered inappropriate to use. \\
\hline \multirow[t]{5}{*}{$\begin{array}{l}\text { Location \& type } \\
\text { of surgery }\end{array}$} & $\begin{array}{l}\text { - All locations in the colon (proximal, ascending, transverse, descending, sigmoid and } \\
\text { rectum) are considered appropriate. }\end{array}$ \\
\hline & $\begin{array}{l}\text { - The small intestine should not be used for research purposes regarding anastomotic } \\
\text { healing in the lower GIT. }\end{array}$ \\
\hline & $\begin{array}{l}\text { - A resection is considered appropriate for constructing an anastomosis; no } \\
\text { consensus was reached on using transection. }\end{array}$ \\
\hline & $\begin{array}{l}\text { - Depending on the animal model, both open and laparoscopic surgery are considered } \\
\text { appropriate. }\end{array}$ \\
\hline & $\begin{array}{l}\text { - Interrupted sutures, running sutures (in all animals) or staplers (in the pig model) } \\
\text { are considered appropriate to construct an anastomosis. }\end{array}$ \\
\hline \multirow[t]{4}{*}{$\begin{array}{l}\text { Macroscopic } \\
\text { outcome }\end{array}$} & $\begin{array}{l}\text { - Anastomotic leakage should always be an outcome, preferably with different grades } \\
\text { of leakage (small/large abscesses, faecal peritonitis, complete dehiscence). }\end{array}$ \\
\hline & $\begin{array}{l}\text { - The available scoring systems for grades of leakage were all considered } \\
\text { inappropriate by the panel. The Anastomotic Complication Score may provide an } \\
\text { appropriate scoring method for macroscopic outcome, but needs to be evaluated } \\
\text { first. }\end{array}$ \\
\hline & - Adhesions to the anastomotic site are relevant as they might cover signs of leakage. \\
\hline & $\begin{array}{l}\text { Adhesions in the abdominal cavity are less relevant and should only be taken into } \\
\text { account in (anti)adhesion studies. }\end{array}$ \\
\hline \multirow[t]{3}{*}{$\begin{array}{l}\text { Histological } \\
\text { assessment }\end{array}$} & $\begin{array}{l}\text { - Histological assessment is very valuable and considered as an appropriate outcome } \\
\text { measure, especially in healing studies. }\end{array}$ \\
\hline & $\begin{array}{l}\text { - Hematoxylin-Eosine staining, Masson's trichrome staining and Pico Sirius Red } \\
\text { staining are all considered appropriate for histological assessment. }\end{array}$ \\
\hline & $\begin{array}{l}\text { - No specific histological score is considered appropriate for microscopic evaluation } \\
\text { of the anastomosis; most important is the comparison with a control group. }\end{array}$ \\
\hline \multirow{3}{*}{$\begin{array}{l}\text { Mechanical and } \\
\text { biochemical } \\
\text { outcome } \\
\text { measures }\end{array}$} & - Both bursting pressure and tensile strength are considered appropriate \\
\hline & $\begin{array}{l}\text { measurements for anastomotic strength. These measurements can be compared } \\
\text { within one experiment, but due to heterogeneity not between different } \\
\text { experiments. }\end{array}$ \\
\hline & $\begin{array}{l}\text { - Additional outcome measures such as hydroxyproline content, amount of collagen, } \\
\text { specific (immunohistochemistry) stainings, ELISA, qPCR are not considered } \\
\text { appropriate for specific anastomotic measurements but can provide information to } \\
\text { answer specific research questions. }\end{array}$ \\
\hline Animal testing \& & - Blinding and randomisation should be used and reported in animal studies \\
\hline \multirow[t]{2}{*}{ Welfare } & $\begin{array}{l}\text { - Detailed information on analgesia, anaesthesia, antibiotics, antiseptic measures, } \\
\text { intestinal segment involved, surgical technique, anastomotic complications as well } \\
\text { as animal welfare is considered appropriate to report in studies. Many panel } \\
\text { members suggested providing this as supplementary (online) data to the } \\
\text { manuscript. }\end{array}$ \\
\hline & - ARRIVE guidelines are appropriate to follow and contribute to standardisation 40 \\
\hline
\end{tabular}


- An online registration of study protocols is considered appropriate for animal research.

\section{Summary of items on which consensus was reached}

In this study consensus was reached amongst researchers studying intestinal anastomoses in animals. The main result is that the selection of an animal model depends on the research question and there is no 'one size fits all'. Consensus was reached that mouse, rat and pig models are considered appropriate models but dog and rabbit models should no longer be used for research on bowel anastomoses in the lower GIT.

The main outcome of the study (anastomotic healing/leakage) should always be evaluated macroscopically, where currently used scores were not considered appropriate enough. The Anastomotic Complication Score as proposed by a panel member may provide an objective scoring measure. Obviously, this new score needs to be evaluated in the experimental setting to obtain information about veracity and/or inter-observer variation, but it does seem to be a promising tool. Bursting pressure (or tensile strength) together with histological evaluation provides further information about the anastomosis. Additional analyses can be helpful to answer specific research questions but are not (yet) considered appropriate as surrogate markers for anastomotic healing. Reporting on animal testing and welfare is still not detailed enough in current literature and ARRIVE guidelines should be followed as much as possible.

A summary of items on which consensus was reached can be found in Table 2.4.

\section{Discussion points - items lacking consensus}

The first topic of debate was the use of small animals. Many panel members felt that they were appropriate to use when systemic interventions are tested, but when interested in a local device a larger animal is preferred. Although it is obvious to prefer to test devices intended for human use in an animal model of comparable size, testing a local device in a rat model is also considered acceptable. Mice should not be used to answer research questions on local devices. Another topic of debate was the use of mice and rats as models for healing or leakage. Many panel members felt that rats cannot be used as a model for AL since they are more resistant to infections and show hardly any clinical signs while other panel members have been using rats for this purpose for many years with very good results. Even though the rat is a validated model for both anastomotic healing and leakage, there are still opponents that claim that a rat is not suitable for this purpose, mostly based on own experiences. There was also disagreement on the consideration of practical 
ease in large animal models. Some believe that they are difficult in terms of anesthesia and housing, while others find them rather easy to handle and do not see any practical disadvantages.

For clinical translation to the human setting, all panel members agreed that the pig was the best-suited model in the pre-clinical setting. However, the use of mice as a clinically relevant model was also suggested, because it might mimic clinical AL better than the rat model. Despite solid arguments and a clinical scoring system proposed by one of the panel members, there was no consensus on the use of small animal models for clinical translation. The proposition to first test a hypothesis in a small animal model (mouse, rat, rabbit) and then use a large animal model (pig or dog) to make it more clinically transferable was not agreed upon and is therefore not recommended.

\section{Discussion}

The frequency of studies that have used an animal model to investigate anastomotic leakage/healing in the lower gastrointestinal tract has increased considerably over the past decade, despite implementation of the 3R principle of Replacement, Reduction and Refinement. As shown in a recent systematic review, reporting quality of these studies is poor and frequently insufficient 4 . Furthermore, a wide variability of animal models and measurement outcomes is used. This study aimed to reach consensus on the most appropriate animal models, outcome measures and animal welfare in research on anastomoses in the lower gastrointestinal tract.

This project used the RAND/UCLA Appropriateness method (RAM) to develop consensus-based recommendation for the use of animal model in research on anastomoses in the lower gastrointestinal tract. While other methods can be used, the RAM is often used in biomedical research and considered as a solid method to use as it has strict parameters to what is defined as consensus.

All principal investigators of the studies included in the literature review were contacted, and the 35 panel members were responsible for $46 \%$ of these studies. The authors that became panel members in this project were enthusiastic about the subject and working in one of the major research groups worldwide involved in experimental research regarding bowel anastomoses. Although this could have lead to selection bias, our approach is more objective than the 'snowball method' in which experts are asked to provide email addresses of other experts. This method is also being used to form an expert panel in consensus studies ${ }^{41}$. Even though the number of panel members is an intrinsic limitation of any consensus project, we 
consider the panel to be a valid representation for researchers that perform animal research on bowel anastomoses in the lower gastrointestinal tract.

We invited authors from studies of the last decade, who are still publishing on this topic. It became clear that they sought to persuade other panel members with their arguments, especially at the beginning of the project. During the second round, all panel members were given their own answers in respect to the answers of the panel as well as arguments provided by other panel members. Obviously, all researchers are convinced of their own methods, believing that their models and techniques are best suited. However, during the project panel members opened up for discussion, making it possible to indeed reach consensus and come up with suggestions and recommendations that can be a useful tool for directing future research.

In 2010 the NC3Rs Reporting Guidelines Working Group published the ARRIVE guidelines for reporting in vivo experiments in animals ${ }^{42}$. While considered appropriate to follow by the panel members in this study, few studies have actually used them to report animal research ${ }^{43}$. Panel members suggested that although these guidelines can contribute to an increase in standardization, and thus can be useful, they are also very detailed and complete. Most of the information required by the guidelines should be available in an online supplementary data section instead of in the manuscript. The panel also felt that it was appropriate to use an online registration for study protocols regarding animal research, (comparable to clinicaltrial.gov in humans) which creates complete transparency. Moreover, they suggested standardised protocol online, per animal model, with guidelines to follow when performing animal research. More transparency in the methods would lead to refinement and reduction in animal experiments due to knowledge on teething problems experienced by others, leading to a decrease in learning time of each model. Also, innovative methods such as intestinal organoids or the use of human tissue that can replace animal models should be further investigated to reduce the use of animals, according to the $3 \mathrm{R}$-principle ${ }^{44}$.

We recommend that future animal research that focuses on intestinal anastomosis should be conducted in either a mouse, rat or pig model and provide detailed information on analgesia, anesthesia, antibiotics, antiseptic measures, intestinal segment involved, surgical technique, anastomotic complications as well as animal welfare. The ARRIVE guidelines should be followed more stringently to increase transparency in animal research. A publicly available online registry together with standardized protocols per animal model are can aid in advancing the field of animal research on bowel anastomoses. 


\section{References}

1. McArdle CS, McMillan DC, Hole DJ. Impact of anastomotic leakage on long-term survival of patients undergoing curative resection for colorectal cancer. Br J Surg. 2005;92(9):1150-1154.

2. Khan AA, Wheeler JMD, Cunningham C, George B, Kettlewell M, Mortensen NJM. The management and outcome of anastomotic leaks in colorectal surgery. Colorectal Dis. 2008;10(6):587-592.

3. Paun BC, Cassie S, MacLean AR, Dixon E, Buie WD. Postoperative complications following surgery for rectal cancer. Ann of Surg. 2010;251(5):807-818.

4. Yauw STK, Wever KE, Hoesseini A, Ritskes-Hoitinga M, van Goor H. Systematic review of experimental studies on intestinal anastomosis. Br J Surg. 2015;102(7):726-734

5. Hagerman GF, Gaertner WB, Ruth GR, Potter ML, Karulf RE. Bovine Pericardium Buttress Reinforces Colorectal Anastomoses in a Canine Model. Dis Colon Rectum. 2007;50(7):1053-1060.

6. Hoeppner J, Crnogorac V, Marjanovic G, Jüttner E, Keck T, Weiser H-F, et al. Small intestinal submucosa for reinforcement of colonic anastomosis. Int J Colorectal Dis. 2009;24(5):543-550.

7. Gaertner WB, Hagerman GF, Potter MJ, Karulf RE. Experimental evaluation of a bovine pericardium-derived collagen matrix buttress in ileocolic and colon anastomoses. J Biomed Mater Res Part B Appl Biomater. 2010;92(1):48-54.

8. Lauder CIW, Strickland A, Maddern GJ. Use of a modified chitosan-dextran gel to prevent peritoneal adhesions in a porcine hemicolectomy model. J Surg Res. 2012;176(2):448-454.

9. Fajardo AD, Amador-Ortiz C, Chun J, Stewart D, Fleshman JW. Evaluation of bioabsorbable seamguard for staple line reinforcement in stapled rectal anastomoses. Surg Innov 2012;19(3):288-294.

10. Sánchez-De Pedro F, Moreno-Sanz C, Morandeira-Rivas A, Tenías-Burillo JM, Alhambra-Rodríguez De Guzmán C. Colorectal anastomosis facilitated by the use of the LigaSure ${ }^{\circledR}$ sealing device: comparative study in an animal model. Surg Endosc. 2013;28(2):508-514.

11. Kanellos I, Mantzoros I, Goulimaris I, Zacharakis E, Zavitsanakis A, Betsis D. Effects of the use of fibrin glue around the colonic anastomosis of the rat. Tech Coloproctol. 2003;7(2):82-84.

12. Kanellos I, Mantzoros I, Demetriades H, Kalfadis S, Kelpis T, Sakkas L, et al. Healing of colon anastomoses covered with fibrin glue after immediate postoperative intraperitoneal administration of 5-fluorouracil. Dis Colon Rectum. 2004;47(4):510-515.

13. Nordentoft T, Rømer J, Sørensen M. Sealing of Gastrointestinal Anastomoses with a Fibrin GlueCoated Collagen Patch: A Safety Study. J Invest Surg. 2007;20(6):363-369.

14. Hoeppner J, Wassmuth B, Marjanovic G, Timme S, Hopt UT, Keck T. Anastomotic sealing by extracellular matrices (ECM) improves healing of colonic anastomoses in the critical early phase. J Gastrointest Surg. 2010;14(6):977-986.

15. Giuratrabocchetta S, Rinaldi M, Cuccia F, Lemma M, Piscitelli D, Polidoro P, et al. Protection of intestinal anastomosis with biological glues: an experimental randomized controlled trial. Tech Coloproctol. 2011;15(2):153-158.

16. Schreinemacher MH, Bloemen JG, Heijden SJ, Gijbels MJ, Dejong CH, Bouvy ND. Collagen fleeces do not improve colonic anastomotic strength but increase bowel obstructions in an experimental rat model. Int J Colorectal Dis. 2011;26(6):729-735.

17. van der Vijver RJ, van Laarhoven CJHM, Ben M de Man, Lomme RMLM, Hendriks T. The effect of fibrin glue on the early healing phase of intestinal anastomoses in the rat. Int J Colorectal Dis. 2012;27(8):1101-1107.

18. Slieker JC, Vakalopoulos KA, Komen NA, Jeekel J, Lange JF. Prevention of leakage by sealing colon anastomosis: experimental study in a mouse model. J of Surg Res. 2013;184(2):819-824.

19. Ulmer TF, Stumpf M, Rosch R, Junge K, Binnebösel M, Trotha KTV, et al. Suture-Free and Mesh Reinforced Small Intestinal Anstomoses: A Feasibility Study in Rabbits. J Invest Surg. 2013;26(4):210-216.

20. Marques e Silva S, Ferreira VMM, Carneiro FP, Feres O, de Oliveira PG, de Sousa JB. Effects of bromopride on the healing of left colon anastomoses of rats. Rev Col Bras Cir. 2011;38(6):429-434. 
21. Pramateftakis MG, Kanellos D, Mantzoros I, Despoudi K, Raptis D, Angelopoulos S, et al. Intraperitoneally administered irinotecan with 5-fluorouracil impair wound healing of colonic anastomoses in a rat model: an experimental study. Tech Coloproctol. Springer Milan; 2011;15(S1):121-125.

22. Cetinkaya K, Dinc S, Gulcelik MA, Renda N, Ustun H, Caydere M, et al. Granulocyte macrophagecolony stimulating factor improves impaired anastomotic wound healing in rats treated with intraperitoneal mitomycin-C. Surg Today. 2005;35(4):290-294.

23. Parra-Membrives P, Ruiz-Luque V, Escudero-Severín C, Aguilar-Luque J, Méndez-García V. Effect of Pentoxifylline on the Healing of Ischemic Colorectal Anastomoses. Dis Colon Rectum. 2007; 50(3):369-375.

24. Vasiliadis K, Pramateftakis MG, Blouhos K, Mantzoros I, Koliakos G, Zaraboukas T, et al. Effect of iloprost on impaired anastomotic healing caused by 5 -fluorouracil plus leucovorin. Dis Colon Rectum. 2007;50(6):899-907.

25. Binnebösel M, Junge K, Kaemmer DA, Krones CJ, Titkova S, Anurov M, et al. Intraperitoneally applied gentamicin increases collagen content and mechanical stability of colon anastomosis in rats. Int J Colorectal Dis. 2009;24(4):433-440.

26. Tingstedt B, Nehéz L, Lindman B, Andersson R. Effect of Bioactive Polypeptides on Leaking Large Bowel Anastomosis and Intestines in the Rat. J Invest Surg. 2007;20(4):229-235.

27. Wang Y, Cai X, Jin R, Liang Y, Huang D, Peng S. Experimental Study of Primary Repair of Colonic Leakage with a Degradable Stent in a Porcine Model. J Gastrointest Surg. 2011;15(11):1995-2000.

28. Bloemen JG, Schreinemacher MH, de Bruine AP, Buurman WA, Bouvy ND, Dejong CH. Butyrate enemas improve intestinal anastomotic strength in a rat model. Dis Colon Rectum. 2010;53(7):1069-1075.

29. Binnebösel M, Grommes J, Koenen B, Junge K, Klink CD, Stumpf M, et al. Zinc deficiency impairs wound healing of colon anastomosis in rats. Int J Colorectal Dis. 2009;25(2):251-257.

30. Velde te EA, Voest EE, van Gorp JM, Verheem A, Hagendoorn J, Gebbink MF, et al. Adverse effects of the antiangiogenic agent angiostatin on the healing of experimental colonic anastomoses. Ann Surg Oncol. 2002;9(3):303-309.

31. van der Vijver RJ, van Laarhoven CJHM, Lomme RMLM, Hendriks T. Paracetamol does not compromise early wound repair in the intestine or abdominal wall in the rat. Anesth Analg. 2012; 115(6):1451-1456.

32. Klein M, Krarup PM, Kongsbak MB, Ågren MS, Gögenur I, Jorgensen LN, et al. Effect of Postoperative Diclofenac on Anastomotic Healing, Skin Wounds and Subcutaneous Collagen Accumulation: A Randomized, Blinded, Placebo-Controlled, Experimental Study. Eur Surg Res. 2012;48(2):73-78.

33. Pommergaard HC, Rosenberg J, Schumacher-Petersen C, Achiam MP. Choosing the best animal species to mimic clinical colon anastomotic leakage in humans: a qualitative systematic review. Eur Surg Res. 2011;47(3):173-181.

34. Fitch K, Bernstein SJ, Aguilar MD, Burnand B, LaCalle JR. The RAND/UCLA Appropriateness Method User's Manual. 2001.

35. Sinha IP, Smyth RL, Williamson PR. Using the Delphi Technique to Determine Which Outcomes to Measure in Clinical Trials: Recommendations for the Future Based on a Systematic Review of Existing Studies. PLoS Med. 2011;8(1):e1000393.

36. Moossdorff M, van Roozendaal LM, Strobbe LJA, Aebi S, Cameron DA, Dixon JM, et al. Maastricht Delphi consensus on event definitions for classification of recurrence in breast cancer research. J Natl Cancer Inst. 2014 Nov 7;106(12).

37. Wenger FA, Szucsik E, Hoinoiu BF, Ionac M, Walz MK, Schmid KW, et al. A new anastomotic leakage model in circular double stapled colorectal anastomosis after low anterior rectum resection in pigs. J Invest Surg. 2013;26(6):364-372.

38. Bedirli A, Yucel D, Ekim B. Laparoscopic Anterior Resection: New Anastomosis Technique in a Pig Model. JSLS. 2014;18(3):e2014.00345.

39. Durães L de C, Durães EFR, Freitas PFS de, Carvalho FANO de, Carvalho SA, Sousa JB de. A new proposal for laparoscopic left colectomy in a rat model. Acta Cir Bras. 2013;28(4):239-244. 
40. Tan KY, Thant Z, Tan P. Transanal radical endoscopic resection of the rectum on a swine model: a surviving model. Asian J Endosc Surg. 2011;4(4):196-198.

41. O'Leary DP, Lynch N, Clancy C, Winter DC, Myers E. International, Expert-Based, Consensus Statement Regarding the Management of Acute Diverticulitis. JAMA Surg. 2015;150(9):899-904.

42. NC3Rs Reporting Guidelines Working Group. Animal research: reporting in vivo experiments: the ARRIVE guidelines. J Physiol. 2010;588(Pt 14):2519-2512

43. Baker D, Lidster K, Sottomayor A, Amor S. Two years later: journals are not yet enforcing the ARRIVE guidelines on reporting standards for pre-clinical animal studies. PLoS Biol. 2014; 12(1):e1001756.

44. Sato T, Clevers H. Growing self-organizing mini-guts from a single intestinal stem cell: mechanism and applications. Science. 2013;340(6137):1190-1194. 


\section{CHAPTER}

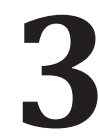

\section{MALDI Imaging: a promising tool in elucidating the pathophysiology of colorectal anastomotic leakage}

Joanna W.A.M. Bosmans, Audrey C.H.M. Jongen, Berta Cillero-Pastor, Gert B. Eijkel, Joep P.M. Derikx, Nicole D. Bouvy, Shane R. Ellis, Ron M. Heeren 
Chapter 3 


\section{Introduction}

Colorectal anastomotic leakage is the most dreaded complication after colorectal surgery because of its high morbidity and even - despite improved postoperative care - mortality ${ }^{1}$. The incidence of anastomotic leakage has remained stable over the last decades despite very extensive research into risk factors, perioperative strategies and intra-operative interventions ${ }^{2}$. The pathophysiology of anastomotic leakage is largely unknown ${ }^{3}$ and we strongly advocate that this should be elucidated prior to conducting intervention studies ${ }^{4}$. Despite some evidence regarding risk factors and some insight in the anastomotic healing process that involves the influx of inflammatory cells, interaction with intestinal microbiota and matrix metalloproteinases, it remains a challenge to study such complex mechanistic pathways within biochemical and biological organisms.

Fortunately, rapid technological advances have been made in recent years to address these kinds of challenges. Among these, Mass Spectrometry Imaging (MSI) has emerged as an enabling technique to provide insight into the molecular entities within cells, tissues, and whole-body samples and to understand inherent complexities within biological metabolomes ${ }^{5}$. Mass spectrometry detects molecules, following ionisation, by virtue of the different mass-to-charge $(\mathrm{m} / \mathrm{z})$ rations. Matrix-assisted laser desorption/ionization (MALDI) is the most common approach used for MSI and involves the use of UV-laser desorption of a matrixcoated tissue section to acquire mass spectra at various locations across a sample (typically every 50-100 $\mu \mathrm{m}$ ). With this method, it is possible to assess a tissuespecific spatial molecular distribution without the need for labelling - in contrast to classical histology - allowing the discovery of prognostic markers and enabling the determination of effective (and personalized) therapies ${ }^{6}$. In addition, MALDIimaging is unique as an imaging technique since it can determine the distribution of hundreds of unknown compounds in a single measurement ${ }^{7}$. With MALDI imaging, recent breakthroughs were achieved as it became possible to reveal diseasespecific molecular features. This process is known as disease phenotyping and has already been done in atherosclerosis ${ }^{8}$, renal cell carcinoma ${ }^{9}$ and metastatic endometrial cancer ${ }^{10}$.

In this short communication we will show a new method on imaging the colonic anastomosis after in vivo experiments, indicating this tool as promising in providing new insights that eventually can lead to therapeutic or even preventive measures for anastomotic leakage. 


\section{Methods}

\section{Animals}

A total of 28 Wistar rats with an average body weight of 250 gram were used. Animals were housed at the Central Animal Facilities of the Maastricht University. All animals were provided ad libitum access to food and water, and were cared for according to local standards. Postoperatively, welfare assessment was performed twice daily using a standardized method and animals were given pain medication in case of discomfort. The experimental protocol complied with the Dutch Animal Experimental Act and was approved by the Animal Experimental Committee of Maastricht University Medical Center (DEC2014-120).

\section{Study design}

The primary objective of this study was to investigate if the processes involved in anastomotic healing can be detected by means of MALDI MSI over time. Additionally, we explored if the components of this healing process change in case of anastomotic leakage, in order to identify factors that are essential for both anastomotic healing and anastomotic leakage. Anastomotic healing was investigated in a rat model with a sufficient anastomosis. The colon was transected $2 \mathrm{~cm}$ distal from the cecum and an end-to-end anastomosis was created using 12 interrupted polypropylene 6/0 sutures (Prolene, Ethicon, Johnson \& Johnson, Somerville, NJ). Time points of sacrifice: 6, 12 and 24 hours, 2, 3, 5, and 7 days after creation of the anastomosis, $\mathrm{n}=2$ per time point.

\section{Sample preparation}

Tissue samples (the anastomosis with a $5 \mathrm{~mm}$ margin on both sides and a control sample $2 \mathrm{~cm}$ distal from the anastomosis) were embedded in a $10 \%$ glycerol solution, snap frozen in liquid nitrogen and stored at $-80^{\circ} \mathrm{C}$. The samples were then cut into $10 \mu \mathrm{m}$ sections for MS analysis, and into $4 \mu \mathrm{m}$ sections for conventional staining using a cryomicrotome (CM 1860 UV, Leica Microsystems GmbH, Wetzlar, Germany) set at $-20^{\circ} \mathrm{C}$. $10 \mu \mathrm{m}$ serial sections for MALDI imaging were thaw mounted on Indium-Tin-Oxide (ITO) coated slides (Delta Electronics (Loveland, CO, USA, 4-8 $\Omega$ sq-1), whilst the $4 \mu \mathrm{m}$ slides were thaw mounted on non-conducting tissue slides. The ITO slides coated with $10 \mu \mathrm{m}$ tissue sections for MS analyses were then placed in a desiccator for 15 minutes. Tissue sections were sprayed with norharmane matrix ( $7 \mathrm{mg} / \mathrm{ml}$ in 12 methanol chloroform) using an automated sprayer (TM-Sprayer, HTX Technologies, Carrboro, NC, USA). The samples were dried and stored in the desiccator until MS analysis. 


\section{Mass spectrometry acquisition}

Biomolecules present in the anastomotic and control tissue were desorbed, ionised and separated according to their mass-to-charge $(\mathrm{m} / \mathrm{z})$ ratios using a Waters Synapt G2-Si mass spectrometer (Waters, Manchester, UK) in positive ion mode at a raster size of $100 \mu \mathrm{m}$, and an $\mathrm{m} / \mathrm{z}$ range of 100-2000. Calibration was performed prior to each measurement with red phosphorous. Regions of interest (ROI) were created by using the HDI v1.4 software (Waters). ROI were based on the histological layers of the gut and in specific the mucosal layer in interaction with gut microbiota, both in control and anastomotic tissue. Commensal microbes and pathogenic bacteria colonizing the intestine have the potential to acquire an aggressive and virulent tissue degrading phenotype, resulting in anastomotic breakdown as was shown by both animal and human research ${ }^{11}$.

Each $m / z$ value present in this spectral collection was converted to an image by using dedicated imaging software (see also data analysis). The chemical structure of each ion detected from the tissue surface was identified after its isolation and fragmentation inside the mass spectrometer (tandem mass spectrometry, MS/MS).

\section{Data analysis}

Comparisons between the different time points and between the anastomotic and control tissue of the same animals were conducted using Biomap (Novartis) and principal component analysis (PCA). Total ion count (TIC) was used for spectral normalization in all cases. In short, PCA is an unsupervised statistical method that aims at pooling a maximum amount of variance in a minimum number of independent variables. Data pre-treatment and PCA were performed using our inhouse built ChemomeTricks toolbox for MATLAB version 2014a (The MathWorks, Natick, MA, USA). The peak assignments were performed according to the bibliography and LIPID MAPS software (http://www.lipidmaps.org/tools/ index.html).

\section{Hematoxylin-Eosin staining}

After analyses, the MSI slides were rinsed in 70\% Ethanol for 10 minutes to remove residual matrix. Tissue specimens were then rehydrated with graded alcohol to $\mathrm{H}_{2} \mathrm{O}$. The slides were submerged into haematoxylin for 3 minutes and rinsed with running tap water for 3 minutes. Subsequently, the slides were counterstained in eosin for 30 seconds and rinsed with running tap water for one minute. Finally, the slides were washed in 100\% Ethanol for 2 minutes and dehydrated in xylene for 30 seconds. Optical images were acquired using a MIRAX Desk Scanner (Zeiss, Gottingen, Germany). 


\section{Results}

We focused on differences in lipid profiles (lipidomics) between colonic tissues. Changes in the distribution and density of cellular constituents were visible as a result of changed distribution of membrane lipids (Figure 3.1), possibly resulting from altered metabolism or cell-response in the process of anastomotic healing.

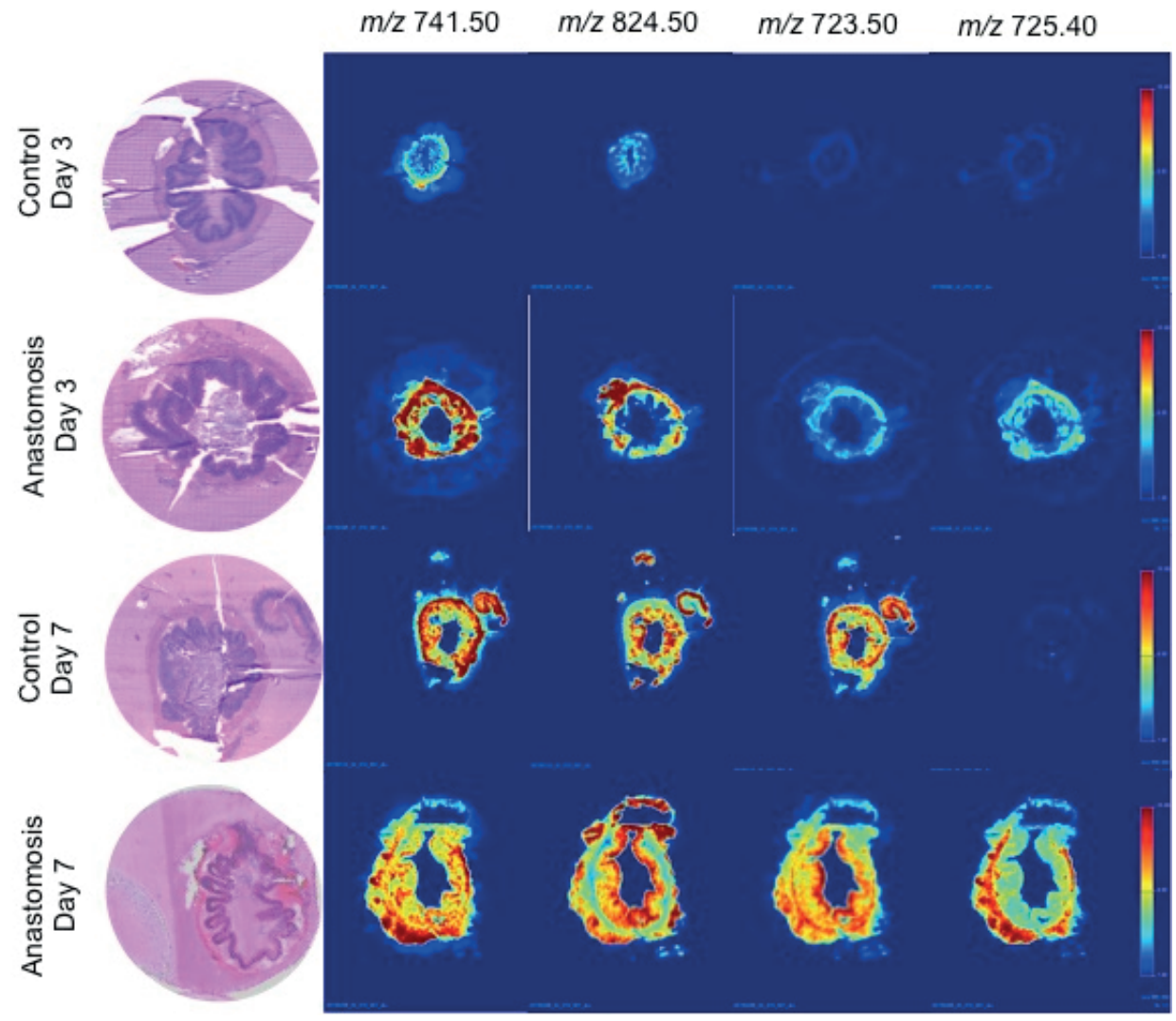

Figure 3.1 Distribution of lipids in anastomotic and control tissue after 3 and 7 days following the construction of an anastomosis in proximal colon of the rat. Intensity scales are set to $50 \%$ maximum and all images were set on the same scale from 0-15. 
Clear differences were observed between different time points and between control and anastomotic tissue (Figures 3.2, 3.3 and 3.4). The signal of $m / z 824.50$ (most likely a phosphatidylcholine $\left(16: 0 / 20: 2^{12}\right)$ showed high correlation with the mucosa and showed an increased expression and signal spreading over time after construction of an anastomosis. The signal of $m / z$ 725.40, which is likely to be sphingomyelin (d18:1/16:013) shows exclusive presence in damaged tissue, i.e. after construction of the anastomosis and an increased expression over time. The control tissue on day 7 shows an expression profile that is more similar to the anastomotic tissue compared to the control tissue on day 3 , which shows relatively low expression of most mass signals. Control tissue that has been exposed to a longer follow-up ( 7 days), shows expression profiles that are more similar to the anastomotic tissue when compared to control tissue after 3 days.

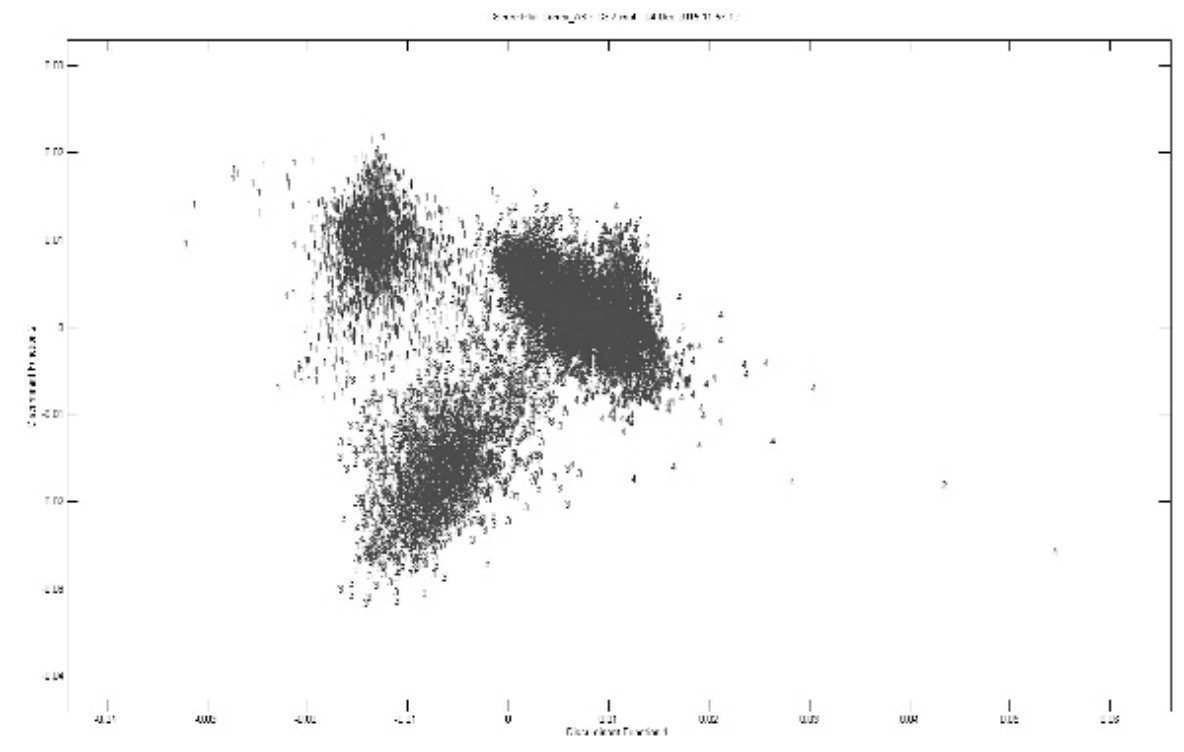

Figure 3.2 Discriminant analysis of cells in control and anastomotic tissue, which displays a clear separation of both conditions as well as in time. 


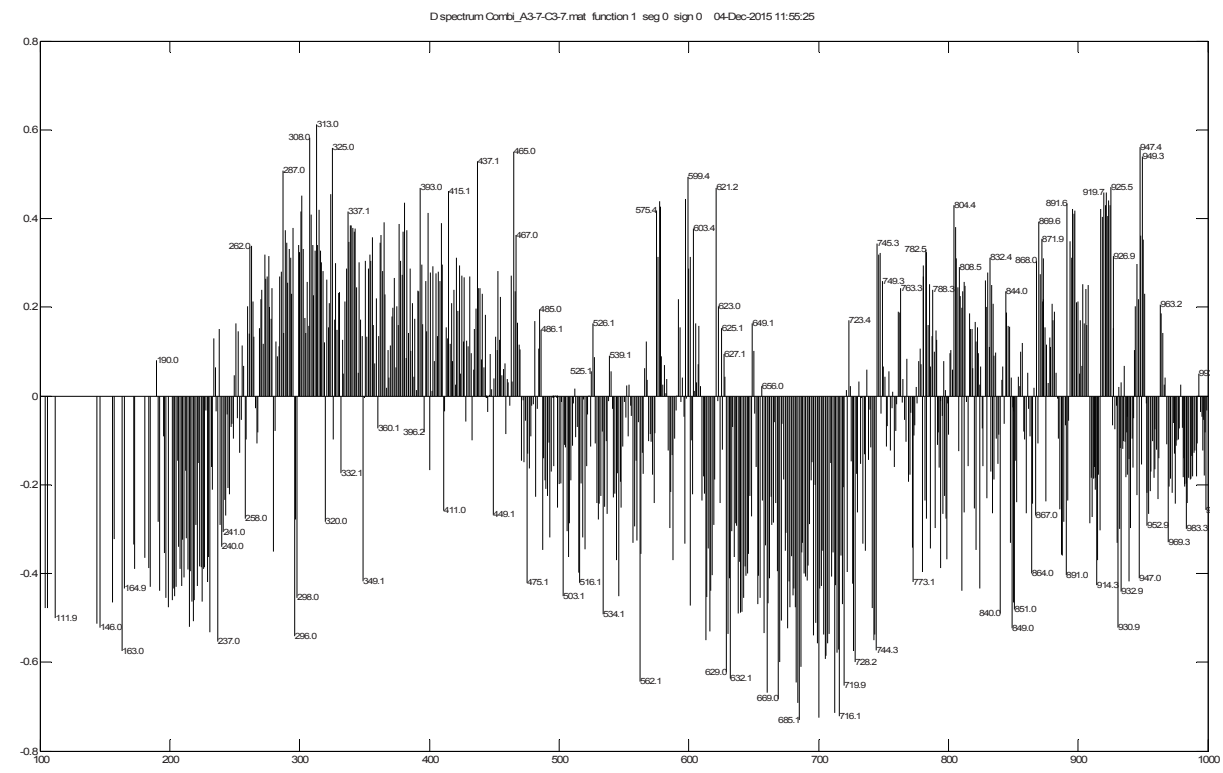

Figure 3.3 Representative combined mass spectrum (DF 1 spectrum) detected by MALDI-MSI from both control and anastomotic tissue at day 3 (negative) and day 7 (positive).

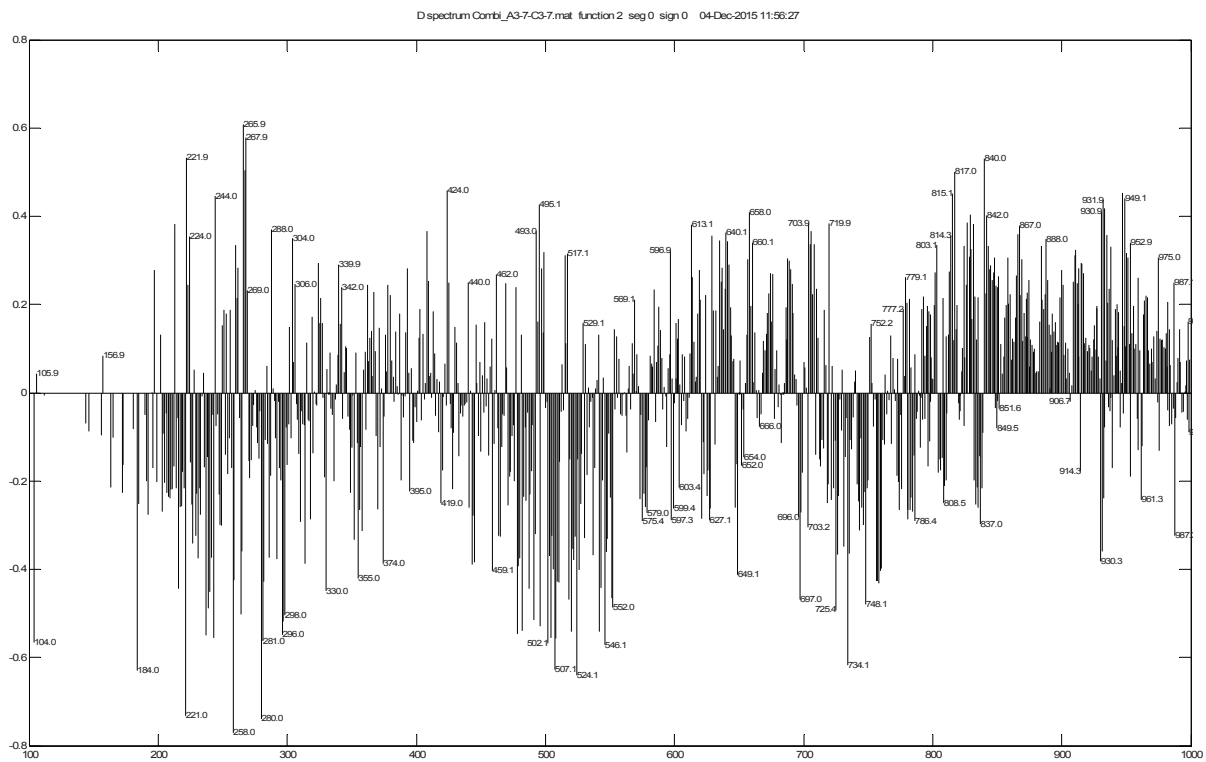

Figure 3.4 Representative combined mass spectrum (DF 2 spectrum) detected by MALDI-MSI from both control (positive) and anastomotic tissue (negative) at day 3 and day 7. 


\section{Discussion and conclusion}

This is a pilot report of a discovery based longitudinal experiment of lipidomic molecular activities in healing and leaking intestinal anastomoses in a rat model. For the first time, localised lipid differences between control and anastomotic tissue has been detected through MALDI-MSI. These differences will be expanded on in the future. The fact that control tissue after 7 days showed similar expression profiles to the anastomotic tissue suggests that the processes involved in healing are not limited to the damaged tissue at the anastomotic site, but instead spread over a larger segment over time. Some mass signals show specific expression in damaged tissue, such as $m / z 725.40$, sphingomyelin ${ }^{13}$. Sphingomyelins have several structural and functional roles in the cell; they are found in the cell membrane and play a role in apoptosis. Recent studies have linked marked alterations in sphingolipid biology to several diseases ${ }^{14,15}$. Particularly detected lipid profiles are specific for pathophysiological processes and can aid in finding new targets for therapy ${ }^{13}$. This has already led to the identification of a mitochondrial key factors in ischemia/reperfusion ${ }^{16,17}$.

This short communication shows that mass spectrometry imaging is a valuable tool to identify molecules that are involved in the normal healing process or in anastomotic leakage. When combined with other modalities (IHC, conventional staining, proteomics, peptidomics, metabolomics), this technique can be used to map the molecular pathways involved in the normal anastomotic healing process, as well pathways involved in leaking which are currently still largely unknown. Current studies from our research group are focusing on unravelling the biochemical processes involved in healing and leaking. Subsequent, specific analyses can be run on the same tissues in order to discover biomarkers for leakage and possibly to develop novel preventive techniques. 


\section{References}

1. Crombe T, Bot J, Messager M, Roger V, Mariette C, Piessen G. Malignancy is a risk factor for postoperative infectious complications after elective colorectal resection. Int J Colorectal Dis. 2016; 31(4):885-894.

2. McDermott FD, Heeney A, Kelly ME, Steele RJ, Carlson GL, Winter DC. Systematic review of preoperative, intraoperative and postoperative risk factors for colorectal anastomotic leaks. $\mathrm{Br} \mathrm{J}$ Surg. 2015;102(5):462-479.

3. Shogan BD, Carlisle EM, Alverdy JC, Umanskiy K. Do we really know why colorectal anastomoses leak? J Gastrointest Surg. 2013;17(9):1698-1707.

4. Bosmans JWAM, Jongen ACHM, Bouvy ND, Derikx JPM. Colorectal anastomotic healing: why the biological processes that lead to anastomotic leakage should be revealed prior to conducting intervention studies. BMC Gastroenterol. 2015;15(1):180.

5. He L. Mass Spectrometry Imaging of Small Molecules. 2014.

6. Schwamborn K, Caprioli RM. Molecular imaging by mass spectrometry — looking beyond classical histology. Nature Reviews Cancer. 2010;10(9):639-646.

7. Cornett DS, Reyzer ML, Chaurand P, Caprioli RM. MALDI imaging mass spectrometry: molecular snapshots of biochemical systems. Nat Methods. 2007;4(10):828-833.

8. Martin-Lorenzo M, Alvarez-Llamas G, McDonnell LA, Vivanco F. Molecular histology of arteries: mass spectrometry imaging as a novel ex vivo tool to investigate atherosclerosis. Expert Rev Proteomics. 2016;13(1):69-81.

9. Alfaro CM, Jarmusch AK, Pirro V, Kerian KS, Masterson TA, Cheng L, et al. Ambient ionization mass spectrometric analysis of human surgical specimens to distinguish renal cell carcinoma from healthy renal tissue. Anal Bioanal Chem. 2016;1-8.

10. Mittal P, Klingler-Hoffmann M, Arentz G, Winderbaum L, Lokman NA, Zhang C, et al. Maldi imaging of primary endometrial cancers reveals proteins associated with lymph node metastasis. Proteomics. 2016 Apr 9.

11. Krezalek MA, Alverdy JC. The role of the microbiota in surgical recovery. Curr Opin Clin Nutr Metab Care. 2016 Jun 23.

12. Hayasaka, T., Goto-Inoue, N., Zaima, N. et al. Lipids, Organ-Specific Distributions of Lysophosphatidylcholine and Triacylglycerol in Mouse Embryo. 2009;44: 837.

13. Rocha B, Cillero-Pastor B, Eijkel G, Bruinen AL, Ruiz-Romero C, Heeren RMA, et al. Characterization of lipidic markers of chondrogenic differentiation using mass spectrometry imaging. Proteomics. 2015;15(4):702-713.

14. Miyamoto S, Hsu C-C, Hamm G, Darshi M, Diamond-Stanic M, Declèves A-E, et al. Mass Spectrometry Imaging Reveals Elevated Glomerular ATP/AMP in Diabetes/obesity and Identifies Sphingomyelin as a Possible Mediator. EBioMedicine. 2016;7:121-134.

15. Melland-Smith M, Ermini L, Chauvin S, Craig-Barnes H, Tagliaferro A, Todros T, et al. Disruption of sphingolipid metabolism augments ceramide-induced autophagy in preeclampsia. Autophagy. 2015;11(4):653-669.

16. Martens JC, Keilhoff G, Halangk W, Wartmann T, Gardemann A, Päge I, Schild L. Lipidomic analysis of molecular cardiolipin species in livers exposed to ischemia/reperfusion. Mol Cell Biochem. 2015;400:253-263.

17. Ji J, Baart S, Vikulina AS, Clark RS, Anthonymuthu TS, Tyurin VA, et al. Deciphering of mitochondrial cardiolipin oxidative signaling in cerebral ischemia-reperfusion. J Cereb Blood Flow Metab. 2015;35(2):319-328. 


\section{Part II}

The use of knockout mice to identify risk factors for anastomotic leakage 


\section{CHAPTER 4}

\section{Cyclooxygenase- 2 is essential for colorectal anastomotic healing}

Kostan W. Reisinger*, Dirk H.S.M. Schellekens*, Joanna W.A.M. Bosmans, Bas Boonen, Maarten F. von Meyenfeldt, Prapto Sastrowijoto, Joep P.M. Derikx, Joep Grootjans, Martijn Poeze

* Both authors contributed equally to this manuscript Annals of Surgery 2016 


\section{Abstract}

\section{Background}

Cyclooxygenase-2 (COX-2) is a key enzyme in gastrointestinal homeostasis. COX-2 inhibitors have been associated with colonic anastomotic leakage.

\section{Methods}

Wildtype, COX-2 knockout and COX-2 heterozygous mice were subjected to a model of colonic anastomotic leakage, and were treated with vehicle, diclofenac, or prostaglandin E2 (PGE2), the most important COX-2 product in the intestine. We assessed anastomotic leakage, mortality, angiogenesis, and inflammation. Furthermore, we investigated the association between anastomotic leakage and a human polymorphism of the COX-2 gene resulting in low COX-2 levels.

\section{Results}

Diclofenac, a nonsteroidal anti-inflammatory drug inhibiting COX-2, increased anastomotic leakage compared to vehicle-treated mice ( $100 \%$ vs $25 \%$, respectively). Similarly, $92 \%$ of COX-2-deficient mice developed anastomotic leakage $(P=0.003)$ compared to WT. PGE2 partly rescued this severe phenotype because only $46 \%$ of PGE2-administered COX-2 knockout mice developed anastomotic leakage $(P=0.02)$. This may be related to decreased neovascularization, because decreased CD31 staining, indicating less blood vessels, was observed in COX-2\% mice ( 2 vessels $/ \mathrm{mm}^{2}$ vs. 6 vessels $/ \mathrm{mm}^{2}$ in controls $(P=0.03)$ ). This effect could partly be reversed by administration of PGE2 to COX-2\% mice. No significant differences in inflammation were found. PTGS2-765G $>C$ polymorphism in humans, associated with reduced COX-2 expression, was associated with higher anastomotic leakage rates.

\section{Conclusions}

COX-2-induced PGE2 production is essential for intestinal wound healing after colonic surgery, possibly via its effects on angiogenesis. These data emphasize that COX-2 inhibitors should be avoided after colonic surgery, and administration of PGE2 might be favorable for a selection of patients. 


\section{Introduction}

The enzyme cyclooxygenase-2 (COX-2), also known as prostaglandin-endoperoxide synthase 2 (Ptgs2), plays an important role in gut homeostasis. In general, cyclooxygenase regulates the conversion of arachidonic acid into prostaglandins, of which prostaglandin E2 (PGE2) is reported to restore intestinal integrity in experimental models of intestinal inflammation and damage ${ }^{1-5}$. COX-2-induced production of prostaglandins in mesenchymal stem cells in the colon have an immunomodulatory role ${ }^{2}$, and these mesenchymal stem cells may thereby act as monitors of the colonic environment. In addition, they might have important functions in colonic wound healing 5 . Furthermore, COX-2 expression is induced in macrophages and myofibroblasts upon exposure to proinflammatory cytokines and bacterial products, leading to proliferation and protection against apoptosis,3,6. Lastly, COX-2 and PGE2 production by endothelial cells are critically involved in vascular endothelial growth factor (VEGF)-induced angiogenesis 7,8 . Data from human studies underline the importance of COX-2 in colonic wound healing as the use of nonsteroidal anti-inflammatory drugs (NSAIDs), especially those with strong COX-2-inhibiting properties, is correlated with the development of anastomotic leakage after colorectal surgery ${ }^{9-12}$. The incidence of anastomotic leakage requiring re-intervention is around $7 \% 13$, making anastomotic leakage the primary complication requiring re-intervention after colonic surgery. Anastomotic leakage carries a high mortality of $15 \%{ }^{14}$. The pathophysiology of anastomotic leakage remains largely unknown and is supposedly multifactorial. Studies showing increased anastomotic leakage due to NSAID use are, however, retrospective in nature and a recent meta-analysis could not prove an unambiguous detrimental effect of NSAIDs ${ }^{15}$. Although these studies suggest to restrict NSAID use in patients with intestinal anastomoses, omission of NSAIDs from postsurgical care in these patients is not yet standard clinical practice ${ }^{16,17}$. Because anastomotic healing requires adequate perfusion of the anastomosis and anastomotic healing seems to be affected by the use of NSAIDS, we set out to investigate the role of COX-2 in the development of anastomotic leakage post colonic surgery.

We provide evidence that COX-2 is essential for neovascularization of the colonic anastomosis and thereby plays a crucial role in colonic anastomotic wound healing. Both pharmacological and genetic disruption of COX-2 function, by use of NSAIDs or COX-2\% mice respectively, increased colonic anastomotic leakage significantly after colonic surgery in mice, which was associated with severely decreased survival.

Importantly, we additionally show that humans with decreased COX-2 expression due to a COX-2 gene polymorphism show increased risk for developing 
anastomotic leakage. These results indicate that COX-2 is essential in colonic anastomotic healing.

\section{Methods}

\section{Mice}

All animal experiments were approved by the Maastricht University Animal Experiments Committee. Mice heterozygous for COX-2 were ordered from Jackson Laboratory (Bar Harbor, ME) to obtain littermate wildtype (WT, n=32), COX-2+/$(n=17)$ and COX-2\% $(n=25)$ mice. For all experiments, COX $-2 \%$ mice were used simultaneously with their wildtype and/or heterozygous littermates.

\section{Model}

A previously described murine model of colonic anastomotic leakage was used and adapted to reach an anastomotic leakage rate of $25 \%$ to $33 \%{ }^{18}$. Briefly, 10 - to 12-week-old mice were anesthetized using isoflurane and were given buprenorphine as analgesic. After a $1 \mathrm{~cm}$ midline laparotomy, the cecum was exteriorized and the right colon was microscopically transected, without damaging blood supply. An end-to-end anastomosis was performed with 7 interrupted sutures (Prolene 8-0, Ethicon, Somerville, NJ). The colon was repositioned and the abdomen was closed in 2 layers of interrupted sutures (Vicryl 4-0, Ethicon, and Ethilon 4-0, Ethicon, respectively). The colon was kept moist with sterile saline during the procedure. Two medically trained researchers that had equal skill and experience with the surgical procedure performed the surgical procedure. There was no difference in the rate of anastomotic leakage between the 2 surgeons. The researchers were blinded for the genotypes at the time of surgery.

\section{Study design}

WT, COX-2 $2^{+/-}$and COX-2/- mice underwent laparotomy with colonic anastomosis, and received vehicle Phosphate-buffered saline (PBS), 16,16-dimethyl PGE2 (dmPGE2, Cayman Chemical, Ann Arbor, MI), a stable analogue of PGE2, or diclofenac sodium (Cayman Chemical) by intraperitoneal injection. Dosage of dmPGE2 was $100 \mathrm{mg} / \mathrm{kg}$ bodyweight, twice daily, and dosage of diclofenac was $10 \mathrm{mg} / \mathrm{kg}$ bodyweight, twice daily ${ }^{5,19}$. This diclofenac dosage was comparable to dosages used in humans when using the dose translation formula published by Reagan-Shaw et al $^{20}$. Vehicle, dmPGE2 and diclofenac treatment was started 1 day prior to laparotomy and continued until the end of the experiments (Figure 4.1). 
Mice were killed at 5 days postoperatively to ensure detection of anastomotic leakage, or when humane endpoints were reached.

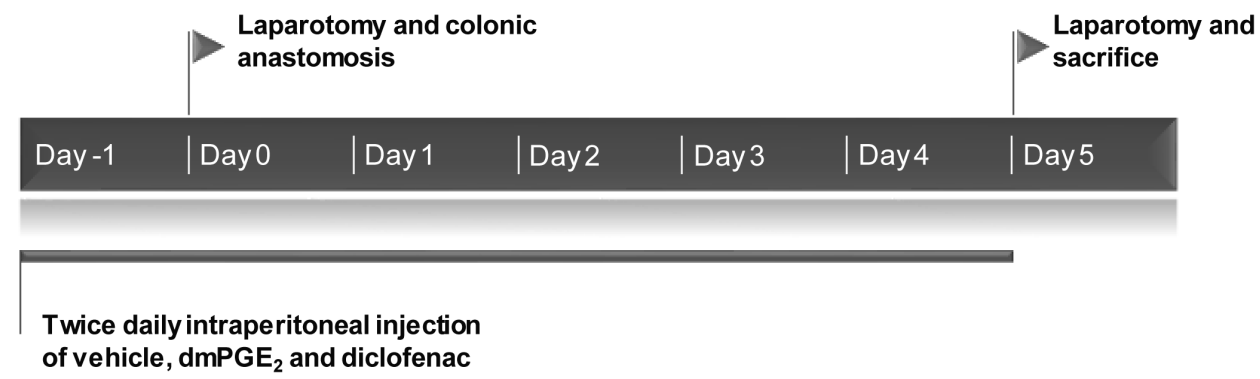

Figure 4.1 Study design: mice received intraperitoneal injections twice daily from one day prior to surgery until sacrifice.

\section{Endpoints}

Anastomotic leakage was defined as either fecal peritonitis or abscess formation around the anastomosis. Two independent investigators who were blinded for genotype at the time of evaluation diagnosed anastomotic leakage.

\section{Tissue preparation}

A $1 \mathrm{~cm}$ segment of colonic tissue surrounding the anastomosis was dissected in longitudinal direction, thereby dividing it into equal parts. One part was fixed overnight in $10 \%$ formalin and embedded in paraffin using standard techniques. The second part was snap frozen in liquid nitrogen and used for mRNA isolation.

\section{Immunohistochemistry}

For immunohistochemistry, sections were deparaffinized in xylene and rehydrated in graded ethanol to distilled water. Endogenous peroxidase activity was blocked using $0.6 \%$ hydrogen peroxide in methanol for 30 minutes. Nonspecific antibody binding was blocked using 5\% bovine serum albumin in PBS [myeloperoxidase (MPO) and CD31 staining] or 10\% normal rabbit serum (MAC-3 staining). Antigen retrieval was required for CD31 immunohistochemistry (IHC) [0.1\% trypsin (Difco Laboratories, Detroit, MI) in $0.1 \% \mathrm{CaCl} 2$ solution for 20 minutes at $37^{\circ} \mathrm{C}$ ] and MAC-3 IHC (10mM sodium citrate, pH 6.0). Sections were incubated with primary antibodies rabbit antihuman MPO antibody (MPO, Dako-Cytomation, Glostrup, Denmark), which cross-reacts with mouse, rat anti-mouse CD31 (BD Pharmingen, Breda, The Netherlands), or rat anti mouse MAC-3 (BD). After washing, biotinconjugated swine anti-rabbit IgG (DakoCytomation), biotinylated rabbit anti-rat IgG 
(DakoCytomation), or biotin-conjugated rabbit anti-rat IgG (Jackson ImmunoResearch, West-Grove, PA) was used for MPO, CD31, and MAC-3 IHC, respectively, followed by incubation with the streptavidin-biotin-HRP system

(DakoCytomation), Brightvision poly HRP anti-rabbit IgG (Immunologic, Duiven, The Netherlands), or streptavidin-biotin-HRP system (Vector Laboratories, Burlingame, CA), respectively. Binding of primary antibody was visualized with 3,30-diaminobenzidine-tetrahydrochloride-dihydrate (Sigma, St Louis, MO) and counterstained with hematoxylin. No staining was detected in slides incubated without primary antibody. Stained sections were photographed by a Nikon eclipse E800 microscope with a Nikon digital camera DXM1200F. The CD31 staining was quantified in a blinded way by 2 observers by counting all CD31 positive vessels at 200x magnification and expressed as the number of vessels per total area $\left(\mathrm{mm}^{2}\right)$ using Image (NIH Software, Bethesda, MD). Staining of MPO was quantified by ImageJ as well and staining of MAC- 3 was quantified by 2 independent blinded observers using computerized morphometry (Leica QWin V3, Cambridge, UK). The results were presented as neutrophils per field of view (MPO) or percentage of positive cells per total tissue area (MAC-3).

\section{Real-time Quantitative Polymerase Chain Reaction}

RNA was isolated from snap-frozen anastomotic tissue samples with AllPrep DNA/RNA/Protein kit (Qiagen, Hilden, Germany) according to the manufacturer's protocol. In short, samples were crushed with a pestle and mortar in liquid nitrogen. Disruption and homogenization of the tissue was performed using an Ultra Turrax Homogeniser (IKA Labortechnik, Staufen, Germany) in lysis buffer containing b-mercaptoethanol (Promega, Madison, WI). RNeasy spin columns were used to bind RNA. Samples were treated with DNAse (Promega) to ensure complete removal of genomic DNA. Columns were washed and RNAwas eluted in RNase-free water. RNA quantity was measured using the NanoDrop spectrophotometer (Thermo Scientific, Wilmington,DE). OnlyRNAsamples with a clearly visible S28 and S18 on agarose gel were considered as intact RNA and were used. Total cDNA was synthesized using the iScriptcDNAsynthesis kit (Bio-Rad, Hercules, CA). qPCR reactions were performed on 10ng cDNA with $300 \mathrm{nM}$ of gene-specific forward and reverse primers and 1X Absolute qPCR SYBR Green Fluorescein Mix (Bioline, London, United Kingdom) using the MyIQ system (Bio-Rad). Sequence of primers for VegfA were: forward TATTCAGCGGACTCACCAGC, reverse CCTCCTCAAACCGTTGGCA). Gene expression levels were calculated with IQ5 software using a $\Delta \mathrm{Ct}$ relative quantification model. The geometric mean of 2 internal control genes (b2-microglobulin and cyclophilin A) was calculated and used as a normalization factor. 


\section{Human tissue collection}

From 148 consecutive patients who underwent oncologic colorectal surgery, paraffin-embedded colonic or rectal tissue sections were collected from the pathology department's database for DNA extraction. In all cases, surgery with primary anastomosis was performed in a single non-academic centre with a dedicated team of colorectal surgeons from January 2010 until August 2011. Data on clinical outcome, including development of anastomotic leakage, had been electronically and prospectively registered in detail for nationwide audit purposes.

\section{DNA isolation from human tissue}

Genomic DNA was obtained from formalin-fixed paraffin-embedded tissue using either a QIAamp DNA FFPE Tissue Kit (Qiagen) or an in-house protocol. In short, 4 tissue sections of $8 \mathrm{~mm}$ per sample were deparaffinized, and samples were lysed under denaturing conditions with proteinase $\mathrm{K}$ (at least $3 \mathrm{~h}$ at $55^{\circ} \mathrm{C}$ ). After centrifuging multiple times, pure genomic DNA was obtained and concentration was determined using Nanodrop. To obtain more purified DNA, all samples were twice precipitated with sodium acetate, both after the amplification and the restriction step.

\section{PTGS2-765G>C Genotyping}

A common promoter variant in the COX-2 gene, $-765 \mathrm{G}>\mathrm{C}$, leads to lower expression of COX-2 as the $-765 \mathrm{C}$ allele, compared with the $-765 \mathrm{G}$ allele, reduces promoter activity ${ }^{21}$. In this study, it was also demonstrated that the $-765 \mathrm{C}$ allele correlates with lower $\mathrm{C}$ reactive protein levels. Therefore, the presence of $-765 \mathrm{G}>\mathrm{C}$ may have significant clinical implications. We hypothesized that the PTGS2-765G>C polymorphism would be associated with an increased risk of anastomotic leakage through lower promoter activity of the COX-2 gene. Amplification of this region was performed using specific forward and reverse primers (CCGCTTCCTTTGTCCATCAG and GGCTGTATATCTGCTCTATATGC, respectively). Subsequently, Acil restriction enzyme (New England Biolabs, Ipswich, MA) was applicated (60 min incubation at $37^{\circ} \mathrm{C}, 20 \mathrm{~min}$ inactivation at $65^{\circ} \mathrm{C}$ ) resulting in both a 188 base pair product and a 118 base pair product in case of a $-765 \mathrm{G}$ allele, or an uncut 306 base pair product in case a $-765 \mathrm{C}$ allele. The presence of homozygous PTGS2-765G $>\mathrm{C}$ polymorphism (-765CC) was then correlated to anastomotic leakage.

\section{Statistics}

Statistical analysis was performed using Prism 5.0 for Windows (Graphpad software, Inc, San Diego, CA) and SPSS 20.0 for Windows (SPSS Inc, Chicago, IL). Normality was tested using Kolmogorov-Smirnov. All continuous variables are 
presented as mean and standard error of the mean and compared using student $\mathrm{t}$ test. Dichotomous variables were compared using chi-square test. Survival was analyzed by logrank test.

\section{Results}

\section{Diclofenac increases colonic anastomotic leakage in an experimental colonic surgery model}

To study whether the observed retrospective human data on colonic anastomic leakage in patients receiving COX-2 inhibitors were also observed in mice, anastomotic leakage rates in mice receiving either vehicle or NSAIDs were determined. Three of 11 mice in the wildtype group developed anastomotic leakage (27\%), which is consistent with previous studies using this experimental model. Intriguingly, all mice in the diclofenac group $(n=9,100 \%)$, however, developed anastomotic leakage, $P=0.001$ (Figure 4.2A). As expected, survival was significantly reduced in the group receiving diclofenac compared with the group receiving vehicle [hazard ratio (HR) 17.9 (95\% confidence interval (CI), 3.7-87.4), $P<0.001$, Figure 4.2B]. These data confirm that NSAIDs in this experimental mouse model also have detrimental effects on anastomotic healing, making it a useful model to study the deleterious effects of NSAIDs in more detail.
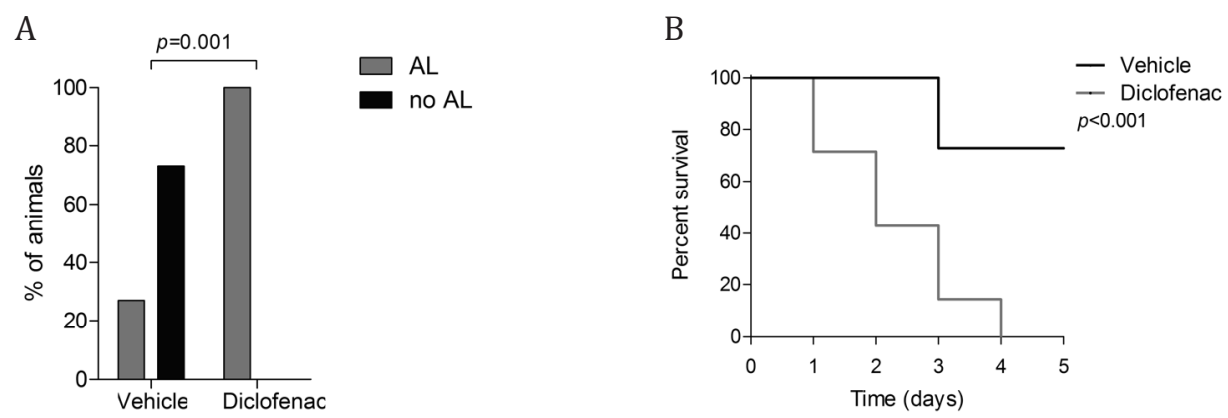

Figure 4.2 Effect of diclofenac on experimental anastomotic healing. A. Incidence of anastomotic leakage in mice treated with vehicle $(n=11)$ and mice treated with diclofenac $(n=9)$. B. Survival of mice treated with vehicle and mice treated with diclofenac. Mice treated with diclofenac showed higher anastomotic leakage incidence and mortality rates.

\section{Cyclooxygenase $\mathbf{2}$ is critically involved in colonic anastomotic healing}

Because we hypothesized that the adverse effects of NSAIDs were particularly a consequence of COX-2 inhibition, we further investigated the selective effects of 
COX-2 in anastomotic healing using COX-2\% mice. Eleven of 12 COX-2\% mice (92\%) had macroscopic leakage compared with only $27 \%$ of WT mice $(P=0.003$, Figure 4.3A). Survival was significantly reduced in COX-2 $\%$ mice compared to WT mice [HR, 3.5 (95\%CI, 1.2-10.3], $P=0.02$, Figure 4.3B]. Because COX-2 is essential for the production of PGE2, which is an important mediator in gut homeostasis, we investigated whether PGE2 administration could rescue COX-2\% from anastomotic leakage. Indeed, supplementation with dmPGE2 halved the anastomotic leakage rate in COX-2\% mice from $92 \%$ ( 11 of 12 mice) to $46 \%$ (6 of 13 mice), $P=0.02$ (Figure 4.4).

A

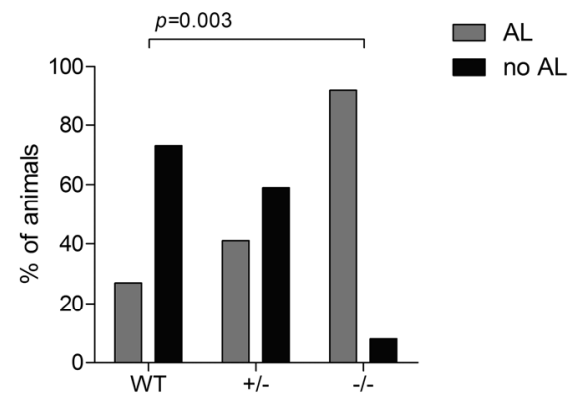

B

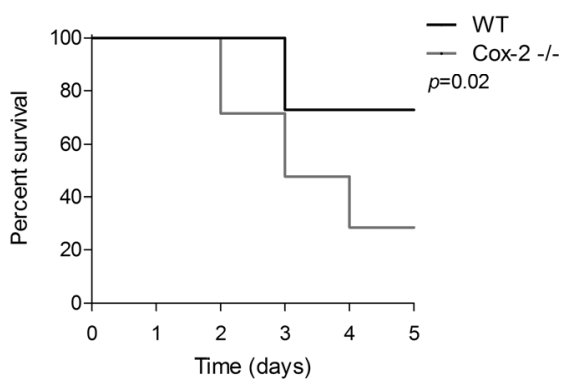

Figure 4.3 Effect of genotype on experimental anastomotic healing. A. Incidence of anastomotic leakage in wildtype $(n=11), \operatorname{COX}-2^{+/-}(n=17)$, and $\operatorname{COX}-2-/-(n=12)$ mice. B. Survival of wildtype and COX- $2 \%$ mice. COX- $2 \%$ mice showed higher anastomotic leakage and mortality rates compared to wildtype mice.

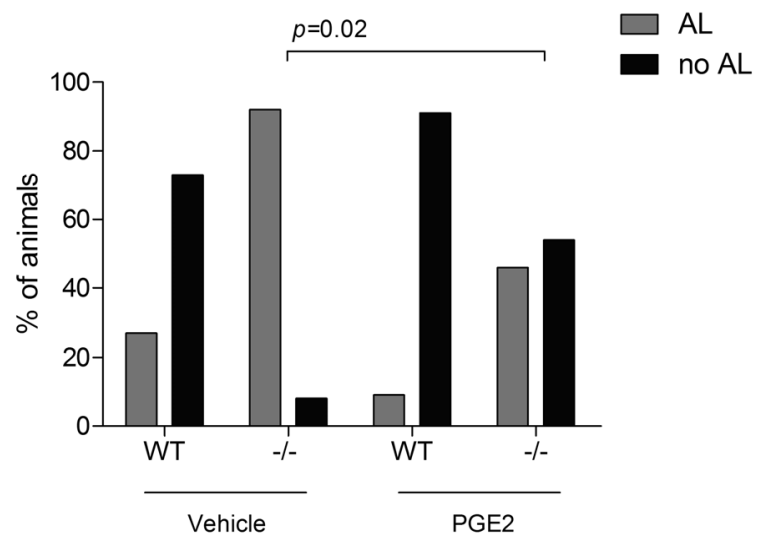

Figure 4.4 Effect of $\mathrm{PGE}_{2}$ administration on anastomotic leakage rates in wildtype (n=12) and COX-2\% $(\mathrm{n}=13)$ mice. $\mathrm{PGE}_{2}$ administration decreased anastomotic leakage rates in $\mathrm{COX}-2 \%$ mice. 


\section{Lack of COX-2 does not lead to inflammatory changes in anastomotic tissue}

Having determined a clear role for COX-2 in anastomotic healing, we set out to dig further into the mechanisms involved in COX-2-dependent impaired anastomotic healing. One of the obvious mechanisms that could be disturbed by blocking COX-2 function is the inflammatory response, which is critical in the first phases of wound healing. In the early phase of wound healing, neutrophils are the predominant cell type; however, no significant differences were observed in influx of MPO-positive cells between WT and COX-2\% mice (Figure 4.5A). On average 148 cells/field of view were found vs. 188 cells/field of view at 100x magnification, WT vs. COX-2\%, respectively (Figure 4.5B). Apart from neutrophils, macrophages are important COX-2 expressing inflammatory cells that also play a major role in wound healing. We observed no significant differences in the presence of MAC-3-positive cells in colonic anastomotic tissue of WT vs. COX-2\% mice. (Figure 4.5A).

A
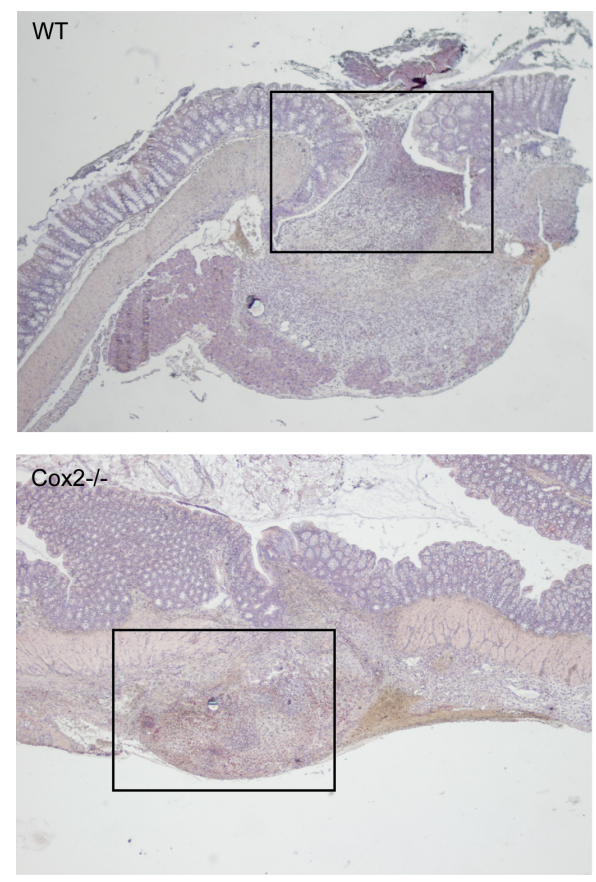

B
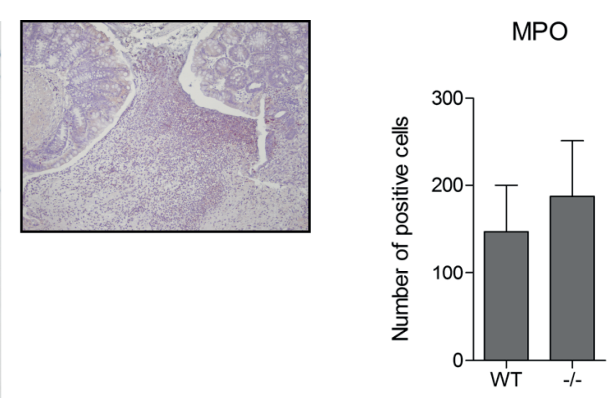

Figure 4.5 Immunohistochemical staining of MPO. A. Sections of colonic anastomotic tissue stained with MPO. Upper panel: wildtype; lower panel: COX-2\%. Magnification 40x, inlay 100x. B. Quantification of MPO staining of wildtype and COX-2 $\%$ mice. Data are presented as mean with SEM (number of positive cells per field of view at 100x magnification). We observed no differences in MPO staining between wildtype and COX-2\% mice. 
On average a $25 \%$ vs. $23 \%$ ratio of positive staining/total tissue area, WT vs. COX-2\%, respectively (Figure 4.6B). These data indicate that local cellular inflammatory responses after colonic surgery are not affected in COX-2\% mice, which may point toward a different underlying factor involved in the impaired anastomotic healing in COX-2\% mice.

\section{A}
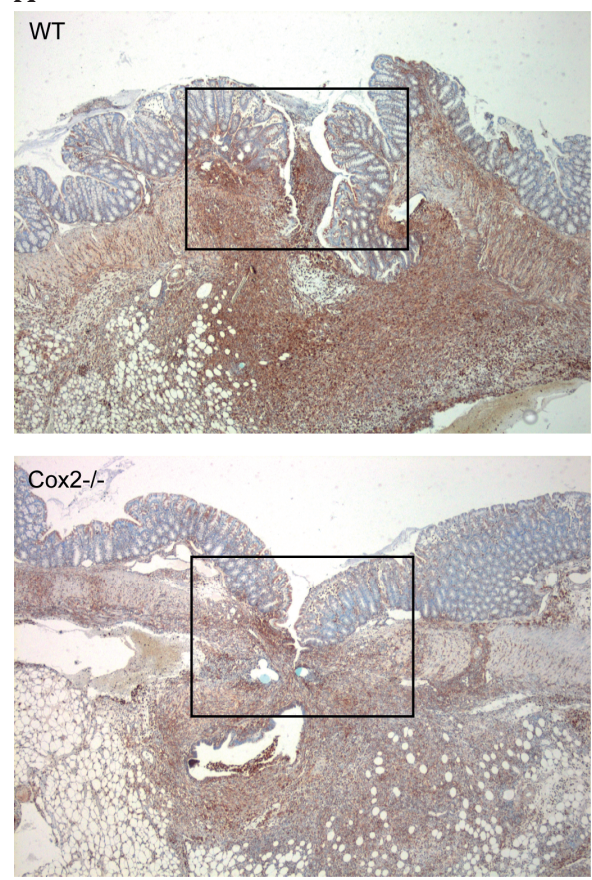

B

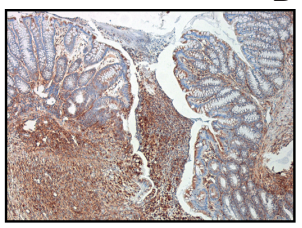

MAC-3

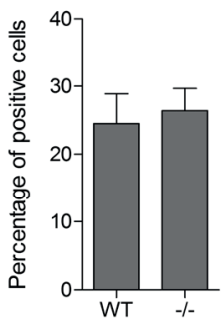

Figure 4.6 Immunohistochemical staining of MAC-3. A. Sections of colonic anastomotic tissue stained with MAC-3. Upper panel: wildtype; lower panel: COX-2\%. Magnification 40x, inlay 100x. B. Quantification of MPO staining of wildtype and COX-2\% mice. Data are presented as mean with SEM (percentage of positive cells per total tissue area). We observed no differences in MAC-3 staining between wildtype and $\mathrm{COX}-2 \%$ mice.

\section{Angiogenesis is impaired in anastomotic tissue of COX-2 knockout mice}

Ischemia of the anastomosis is an important factor associated with the occurrence of anastomotic leakage, and (neo)vascularization of the wound region is critical for adequate wound healing22. Because COX-2 is well known to be involved in angiogenesis, we next assessed whether vascularization of the wound region was impaired in COX-2\% mice. Staining of CD31, an endothelial marker, was markedly reduced in anastomotic tissue of COX-2\% mice compared to control. Quantification 
of the amount of blood vessels showed that only 2 vessels $/ \mathrm{mm}^{2}$ were stained in anastomotic tissue of COX-2\% mice compared to 6 vessels $/ \mathrm{mm}^{2}$ in wildtype mice, 5 days after surgery $(P=0.03$, Figures $4.7 \mathrm{~A}, 4.7 \mathrm{~B})$. This effect could partly be reversed by administration of $\mathrm{dmPGE} 2$ to $\mathrm{COX}-2 \%$ mice, because significantly higher numbers of blood vessels were observed in COX-2/- mice receiving dmPGE2 compared to COX-2\% receiving vehicle (4 vs. 2 vessels $/ \mathrm{mm}^{2}$, respectively, $\mathrm{P}=0.03$, Figure 4.7B). Similarly, anastomotic tissue mRNA levels of VEGF were higher in wildtype mice compared to $\mathrm{COX}-2 \%$ mice $(P=0.02)$, and in COX $-2 \%$ mice receiving dmPGE2 compared to $\mathrm{COX}-2 \%$ mice receiving vehicle $(P=0.03)$.

A
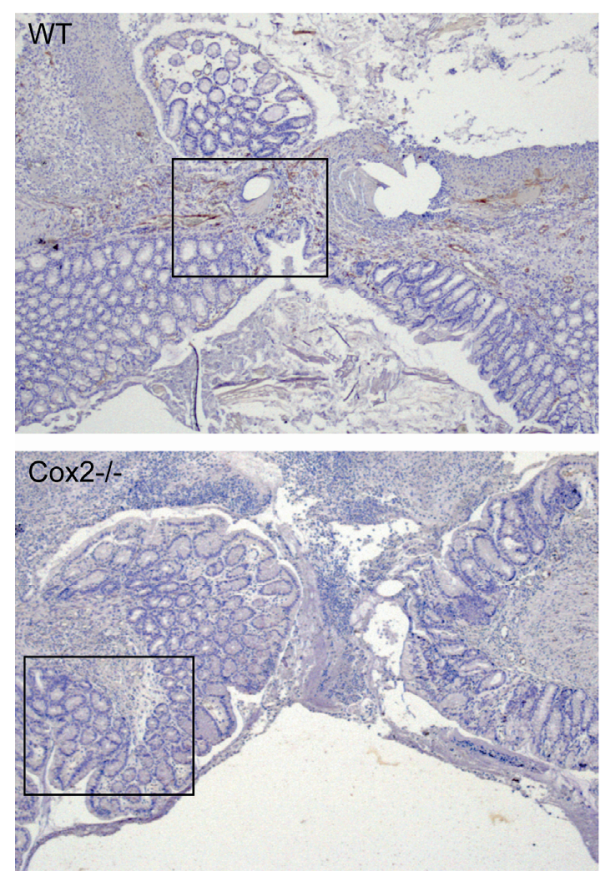
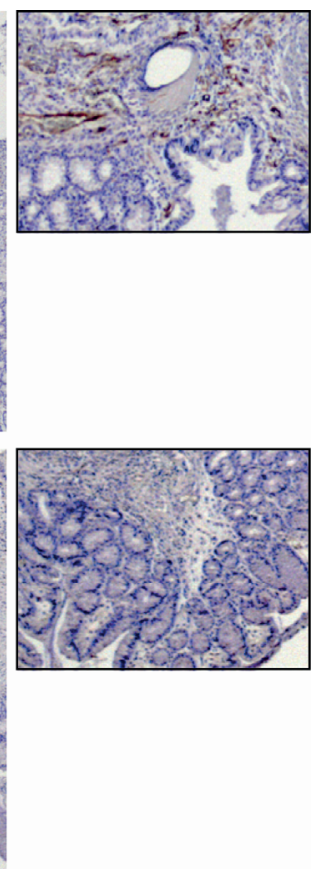

B

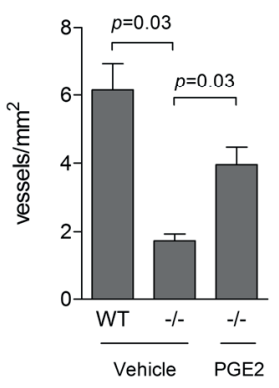

$\mathrm{C}$

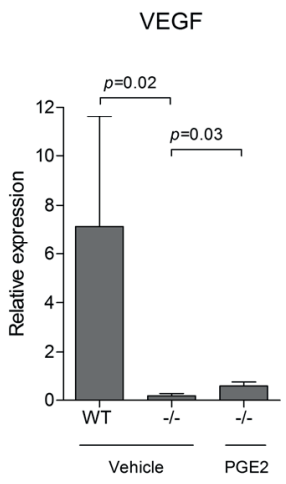

Figure 4.7 COX-2-/- mice exhibit less vascularization in colonic anastomotic tissue. A. Immunohistochemical staining of CD31 on sections of colonic anastomotic tissue. Upper panel: wildtype; lower panel: COX-2\%. Magnification 40x, inlay 100x. B. Quantification of MPO staining of wildtype and COX-2\% mice, and COX-2\% mice receiving PGE2. Data are presented as mean with SEM. CD31 expression was significantly lower in COX-2\% mice compared to wildtype mice and compared to COX-2\% mice receiving PGE2. C. Relative expression of VEGF mRNA in colonic anastomotic tissue of wildtype and COX-2\% mice, and COX- $2 \%$ mice receiving PGE2. Data are presented as mean with SEM. Relative VEGF mRNA expression was significantly lower in COX-2\% mice compared to wildtype mice and compared to COX-2\% mice receiving PGE2. 


\section{Humans with genetically impaired COX-2 expression show high anastomotic leakage incidence}

Of 148 patients undergoing colorectal resection for malignancy, 7 were homozygous for the PTGS2-765G>C polymorphism. Three of 7 (43\%) developed anastomotic leakage compared with 16 of $141(11 \%)$ patients with the $-765 \mathrm{GC}$ or 765GG genotype $(P=0.02$, Figure 4.8).

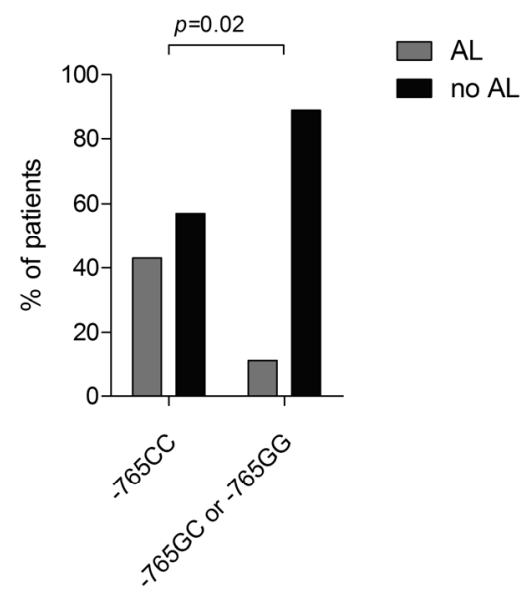

Figure 4.8 Effect of human genotype (a common promoter variant in the COX-2 gene, $-765 \mathrm{G}>\mathrm{C}$ ) on anastomotic leakage incidence. $-765 \mathrm{CC}(\mathrm{n}=7)$ and $-765 \mathrm{GC}$ pooled with $-765 \mathrm{GG}$ $(\mathrm{n}=157)$ are depicted.

\section{Discussion}

This study shows that COX-2 is of critical importance in preventing anastomotic leakage after colonic surgery in a mouse experimental colonic anastomosis model using both a pharmacological and a genetic approach. Mice lacking COX-2 showed increased rates of anastomotic leakage and mortality, which could be partly counteracted by administration of PGE2, supposedly the most important product of COX-2 in preserving gut homeostasis. Intriguingly, PTGS2-765G $>$ C polymorphism in humans, associated with reduced COX-2 expression, was associated with higher anastomotic leakage rates. Impaired angiogenesis in mice lacking COX-2 seems to be at least part of the explanation for increased anastomotic leakage rates and mortality. 
A correlation between the use of COX-2 blocking NSAIDs and impaired anastomotic healing after colorectal surgery has already been established in patients ${ }^{9,11,12}$. However, these data are of retrospective nature and a fundamental role of COX-2 in gut healing after colorectal surgery has never been established. Manieri et al showed that COX-2 is critically involved in mucosal repair after intestinal biopsies ${ }^{5}$. Furthermore, abundant evidence exists showing that COX-2 and its derived prostaglandins stimulate intestinal cancer progression through enhanced angiogenesis and proliferation and by decreasing apoptosis ${ }^{23-25}$. Although these mechanisms should be counteracted to reduce cancer progression, they are indispensable in adequate wound healing. As shown by Binion et al, COX-2 and PGE2 stimulate angiogenesis through VEGF production (by endothelial cells) ${ }^{8}$. Indeed, our study showed that mice lacking COX-2 have significantly lower amounts of CD31+ vessels in anastomotic tissue that was accompanied by lower levels of VEGF mRNA levels and was at least partly restored by PGE2 administration.

The current study has several important implications. As suggested earlier by retrospective human data, the perioperative use of NSAIDs should be avoided in patients undergoing surgery with a bowel anastomosis. Furthermore, patients with mutations in the COX-2 gene may be particularly vulnerable to impaired intestinal wound healing and the subsequent risk of complications after colorectal surgery or other therapeutic hits impairing gut homeostasis. This was underlined in the current study by the increased anastomotic leakage risk in patients carrying 2 alleles of the PTGS2-765G $>C$ polymorphism. Although the prevalence of the homozygous $-765 \mathrm{C}$ gene is reported to be around $3 \%{ }^{26}$, selection and personalized treatment of these patients should be investigated, for example, the administration of prostaglandins such as PGE2 in phases when adequate intestinal healing is crucial.

A number of methodologic remarks must be made when interpreting these observations. As with nearly all animal anastomotic leakage models, in the current model a mechanically insufficient anastomosis was created. However, these models provide an excellent and unique opportunity to study the mechanisms of intestinal wound healing, while also providing clinical readouts, such as anastomotic leakage and mortality. Another limitation was the lack of a random computer-generated multiple-area analysis of our IHC data, which may have biased our observations. Furthermore, there could be differences between inflammatory mediators that were missed. Future studies should address this.

A discrepancy between previous human reports and our used model is that all mice receiving diclofenac developed anastomotic leakage. Although this 
underscores the importance of this pathway in anastomotic healing, such high anastomotic leakage rates were not observed in previous animal studies 27,28 . Blockage of both COX-2 and COX-1 by diclofenac, which is constitutively expressed in colonic tissue ${ }^{29}$, may be the reason for this effect.

The survival of mice with anastomotic leakage in the current study was remarkably low, with most mice dying within several days. For example, higher survival rates are reported for most cecal puncture and ligation protocols, although cecal puncture and ligation protocols inducing high-grade sepsis are reported to have similar survival rates ${ }^{30}$. This might indicate a high degree of necrosis; however, we did not find this on tissue sections.

PGE2 administration did not fully reverse the incidence of anastomotic leakage in COX-2 knockout mice to wildtype levels. This could be explained by the fact that we only used PGE2 to counteract the effects of genetic lack of COX-2. However, other prostaglandins are converted from arachidonic acid by COX-2, as well. In particular, prostacyclin (PGI2) is expressed in colonic tissue and may therefore play a role in anastomotic healing 5 . Another explanation could be that the dose or frequency of PGE2 administration should be increased for optimal effects.

In conclusion, we show that COX-2 inhibition is deleterious for anastomotic healing after colonic surgery, which is mediated at least in part by PGE2 production. Mice lacking COX-2 show higher rates of anastomotic leakage and increased mortality. In addition, angiogenesis is significantly impaired in the absence of COX-2 and PGE2, providing clues for the mechanism by which COX-2 influences anastomotic healing. Importantly, we show that patients with a polymorphism that is associated with decreased COX-2 levels were more likely to develop anastomotic leakage, which further supports our conclusions. We suggest that until proven otherwise, the perioperative use of COX-2 inhibitors in colonic surgery should be replaced by alternative analgesics, and future studies should assess the potential of PGE2 administration prior to colonic surgery to reduce anastomotic leakage rates in a selection of patients. 


\section{References}

1. Blikslager AT, Roberts MC, Rhoads JM, et al. Prostaglandins I2 and E2 have a synergistic role in rescuing epithelial barrier function in porcine ileum. J Clin Invest. 1997;100:1928-1933.

2. Walker MR, Brown SL, Riehl TE, et al. Growth factor regulation of prostaglandin-endoperoxide synthase 2 (Ptgs2) expression in colonic mesenchymal stem cells. J Biol Chem. 2010;285: 5026-5039.

3. Fukata M, Chen A, Klepper A, et al. Cox-2 is regulated by Toll-like receptor-4 (TLR4) signaling: Role in proliferation and apoptosis in the intestine. Gastroenterology. 2006;131:862-877.

4. Brown SL, Riehl TE, Walker MR, et al. Myd88-dependent positioning of Ptgs2-expressing stromal cells maintains colonic epithelial proliferation during injury. J Clin Invest. 2007;117:258-269.

5. Manieri NA, Drylewicz MR, Miyoshi H, et al. Igf2bp1 is required for full induction of Ptgs2 mRNA in colonic mesenchymal stem cells in mice. Gastroenterology. 2012;143:110-121.

6. Singer II, Kawka DW, Schloemann S, et al. Cyclooxygenase 2 is induced in colonic epithelial cells in inflammatory bowel disease. Gastroenterology. 1998;115:297-306.

7. Wang D, Dubois RN. Prostaglandins and cancer. Gut. 2006;55:115-122.

8. Binion DG, Otterson MF, Rafiee P. Curcumin inhibits VEGF-mediated angiogenesis in human intestinal microvascular endothelial cells through COX-2 and MAPK inhibition. Gut. 2008;57: 1509-1517.

9. Gorissen KJ, Benning D, Berghmans T, et al. Risk of anastomotic leakage with non-steroidal antiinflammatory drugs in colorectal surgery. Br J Surg. 2012;99:721-727.

10. Klein M, Andersen LP, Harvald T, et al. Increased risk of anastomotic leakage with diclofenac treatment after laparoscopic colorectal surgery. Dig Surg. 2009;26:27-30.

11. Klein M, Gogenur I, Rosenberg J. Postoperative use of non-steroidal anti-inflammatory drugs in patients with anastomotic leakage requiring reoperation after colorectal resection: cohort study based on prospective data. BMJ. 2012;345:e6166.

12. Holte K, Andersen J, Jakobsen DH, et al. Cyclo-oxygenase 2 inhibitors and the risk of anastomotic leakage after fast-track colonic surgery. Br J Surg. 2009;96:650-654.

13. Snijders HS,WoutersMW, van Leersum NJ, et al. Meta-analysis of the risk for anastomotic leakage, the postoperative mortality caused by leakage in relation to the overall postoperative mortality. Eur J Surg Oncol. 2012;38:1013-1019.

14. McArdle CS, McMillan DC, Hole DJ. Impact of anastomotic leakage on longterm survival of patients undergoing curative resection for colorectal cancer. Br J Surg. 2005;92:1150-1154.

15. Burton TP, Mittal A, Soop M. Nonsteroidal anti-inflammatory drugs and anastomotic dehiscence in bowel surgery: systematic review and metaanalysis of randomized, controlled trials. Dis Colon Rectum. 2013;56:126-134.

16. Gustafsson UO, Scott MJ, SchwenkW, et al. Guidelines for perioperative care in elective colonic surgery: Enhanced Recovery After Surgery (ERAS(R)) Society recommendations. Clin Nutr. 2012;31:783-800

17. Nygren J, Thacker J, Carli F, et al. Guidelines for perioperative care in elective rectal/pelvic surgery: Enhanced Recovery After Surgery (ERAS(R)) Society recommendations. Clin Nutr. 2012;31: 801816.

18. Komen N, van der Wal HC, Ditzel M, et al. Colorectal anastomotic leakage: a new experimental model. J Surg Res. 2009;155:7-12.

19. Takasaki I, Sasaki A, Andoh T, et al. Effects of analgesics on delayed postherpetic pain in mice. Anesthesiology. 2002;96:1168-1174.

20. Reagan-Shaw S, Nihal M, Ahmad N. Dose translation from animal to human studies revisited. FASEB J. 2008;22:659-661.

21. Papafili A, Hill MR, Brull DJ, et al. Common promoter variant in cyclooxygenase-2 represses gene expression: evidence of role in acute-phase inflammatory response. Arterioscler Thromb Vasc Biol. 2002;22:1631-1636.

22. Vignali A, Gianotti L, Braga M, et al. Altered microperfusion at the rectal stump is predictive for rectal anastomotic leak. Dis Colon Rectum. 2000;43:76-82. 
23. Liao X, Lochhead P, Nishihara R, et al. Aspirin use, tumor PIK3CA mutation, and colorectal-cancer survival. N Engl J Med. 2012;367:1596-1606.

24. Rothwell PM, Fowkes FG, Belch JF, et al. Effect of daily aspirin on long- term risk of death due to cancer: analysis of individual patient data from randomised trials. Lancet 2011;377:31-41.

25. Wang D, Dubois RN. Eicosanoids and cancer. Nat Rev Cancer 2010;10:181-93.

26. Ulrich CM, Whitton J, Yu JH, et al. PTGS2 (COX-2) -765G > C promoter variant reduces risk of colorectal adenoma among nonusers of nonsteroidal anti-inflammatory drugs. Cancer Epidemiol Biomarkers Prev 2005;14:616-9.

27. Klein M, Krarup PM, Burcharth J, et al. Effect of diclofenac on cyclooxygenase-2 levels and early breaking strength of experimental colonic anastomoses and skin incisions. Eur Surg Res 2011;46:26-31

28. Inan A, Koca C, Sen M. Effects of diclofenac sodium on bursting pressures of anastomoses and hydroxyproline contents of perianastomotic tissues in a laboratory study. Int J Surg 2006;4:222-7.

29. Tessner TG, Cohn SM, Schloemann S, et al. Prostaglandins prevent decreased epithelial cell proliferation associated with dextran sodium sulfate injury in mice. Gastroenterology 1998;115:874-82.

30. Rittirsch D, Huber-Lang MS, Flierl MA, et al. Immunodesign of experimental sepsis by cecal ligation and puncture. Nat Protoc. 2009;4:31-36. 


\section{CHAPTER 5}

\section{Functional mucous layer and healing of proximal colonic anastomoses in an experimental model}

Joanna W.A.M. Bosmans, Audrey C.H.M. Jongen, George M.H. Birchenough, Elisabeth E.L. Nyström, Marion J.J. Gijbels, Joep P.M. Derikx, N.D. Bouvy, Gunnar C. Hansson Accepted in British Journal of Surgery, 2017 


\section{Abstract}

\section{Background}

Anastomotic leakage (AL) is the most dreaded complication after colorectal surgery causing high morbidity and mortality without its pathophysiology being completely elucidated. Since mucus is a first line of defence against external factors in the gastrointestinal tract, it was investigated if depletion of the structural mucus protein Muc2 is associated with impaired colon anastomotic healing.

\section{Methods}

Mice of different Muc2 genotypes were subjected to a model of proximal colonic AL. Tissues were histologically scored for inflammation, bacterial translocation was determined by qPCR of bacterial 16S rDNA, and epithelial cell damage was determined by assessing serum levels of intestinal fatty acid binding protein.

\section{Results}

Of 22 Muc2 deficient (Muc2\%) mice, 20 developed AL, compared with 7 of 22 control animals $(P<0.001)$. Control mice showed normal healing whereas Muc2\%-showed more inflammation with less collagen deposition and neoangiogenesis. A tendency towards higher bacterial translocation was seen in Muc2\% mesenteric lymph node and spleen. Intestinal fatty acid binding protein levels were significantly higher in Muc2 $\%$ mice compared to controls $(P=0.011)$.

\section{Conclusions}

A functional mucous layer facilitates the healing of colonic anastomoses. 


\section{Introduction}

Anastomotic leakage (AL) is one of the most dreaded complications of colorectal surgery, and leads to high morbidity and mortality ${ }^{1,2}$. AL rates remain high, with reported rates ranging from 1 to almost 20 per cent, despite extensive research, improvement of surgical techniques and the development of fast-track programmes ${ }^{3,4}$. Identified risk factors for AL development, such as male sex, steroid use and location of the tumour, as well as concurrent disease and malnutrition, can help clinicians to determine which patients are at risk ${ }^{5,6}$. However, the underlying pathophysiological mechanisms for AL remain unclear7. A better insight to these mechanisms, and the factors promoting anastomotic healing, is needed to reduce rates of $\mathrm{AL}^{8}$.

The colon is protected by a two-phase mucous layer built around the gelforming MUC2 mucin and a limited number of other components secreted from the goblet cells 9,10 . MUC2 mucin polymers form net-like sheets that, when layered on top of one another, form an inner colonic mucous layer that is impenetrable to bacteria ${ }^{11}$. This layer is about $50 \mu \mathrm{m}$ thick in mice and $200 \mu \mathrm{m}$ in humans, and is quickly renewed ${ }^{12}$. The inner layer is converted to an non-attached outer mucous layer, which is penetrable for colonic bacteria that use this layer as their habitat ${ }^{9,10}$. Muc2 gene-deficient mice lack a functional mucous layer, and have bacteria in direct contact with the epithelial cells in the intestine9. These mice develop colonic inflammation with a severity that depends on the bacterial flora in the animal housing facility.

Among the numerous factors contributing to healing of surgical anastomoses, the intestinal mucus system has been largely overlooked. This is despite the fact that numerous studies have shown that mucus is essential for protection of the epithelium from luminal challenge. For example, it was recently demonstrated that colonic ischaemia resulted in detachment of mucus, which allowed bacteria to come into contact with the epithelium ${ }^{13}$. When blood flow was restored, crypt goblet cells secreted stored mucus, which cleared the bacteria in contact with the epithelium, and re-established homeostasis. Thus, goblet cell mucous secretion plays a central role in regenerating a functional mucous layer that protects the host epithelium ${ }^{14}$.

Mucin secretion influences the capacity of the mucous layer to protect the colon, and altered secretion may result in different physiological consequences ${ }^{15}$. Barcelo and colleagues ${ }^{16}$ demonstrated that mucin secretion can be modulated by luminal factors such as certain algal polysaccharides, uronic acids and short-chain fatty acids (SCFAs). Other factors influencing mucous secretion are prostaglandins and certain bacteria, such as Listeria monocytogenes and Akkermansia muciniphila17,18. 
In addition, prostaglandin (PG) E2, produced by the two isoforms of cyclooxygenase (COX) 1 and 2, can stimulate mucous secretion in both small intestine and proximal colon ex vivo ${ }^{19}$. Non-steroidal anti-inflammatory drugs (NSAIDs) inhibit COX enzymes, leading to a decrease in prostaglandin synthesis ${ }^{20}$. An association between the use of NSAIDs and AL has been described in both experimental and observational studies ${ }^{21-23}$, suggesting an interaction between prostaglandins and anastomotic healing. PGE2 could thus have a positive influence on anastomotic healing, mediated by its effect on mucous secretion.

In the present study, the protective role of a functional mucous layer on AL rates after colonic surgery in mice, and the influence of PGE2 administration on anastomotic wound healing, was investigated.

\section{Methods}

\section{Animals}

All animal experiments were approved by the Gothenburg Animal Experiments Ethics Committee (permit 63-2014), and all experiments were performed in compliance with the directive on the protection of animals used for scientific purposes (2010/63/EU). This study followed the ARRIVE guidelines ${ }^{24}$; Appendix S1 (supporting information) provides more detailed information.

Forty-four mice (mean(s.d.) weight 22.3(3.8) g) of different genotypes (Muc2-/-, $\mathrm{Muc2}^{+/-}$and $\mathrm{Muc2}^{+/+}$) were matched for age (8-20 weeks) and sex when possible, in order to obtain equal distribution within experimental groups (2 groups: Muc2-/versus control (Muc2 $2^{+-}$and Muc2 ${ }^{++}$); 22 mice per group). Additionally, eight Muc2 ${ }^{-/}$mice served as control animals in the quantitative PCR (qPCR) experiment for bacterial translocation, to distinguish between effects induced by surgery and the genotype. The mice had unlimited access to standard mouse chow and water. Welfare of the animals was assessed twice daily using extensive scoring according to Pommergaard and colleagues ${ }^{25}$. Postoperative pain relief treatment with buprenorphine $0.1 \mathrm{mg} / \mathrm{kg}$ subcutaneously (Buprecare®; AST Farma, Oudewater, The Netherlands) was administered when needed.

\section{Study design}

Muc2-/-, Muc2+/- and wild-type (WT; Muc2 ${ }^{+/+}$) mice underwent laparotomy with colonic anastomosis and were killed on at day 3 after the operation. The heterozygous and WT mice were considered control animals (Muc2+), as both of these genotypes determine a normal mucous layer. The primary outcome was $\mathrm{AL}$, 
defined by either faecal peritonitis or abscess formation at the anastomotic site. Macroscopic AL was diagnosed by two independent observers, who were blinded for the genotypes at the time of death.

\section{Model}

The model of AL used was that described previously by Komen and co-workers ${ }^{26}$ and subsequently adapted by Reisinger et al. ${ }^{27}$ to obtain a model that resembles clinical practice. In this model, mice undergo anaesthesia with isoflurane and receive pain treatment with buprenorphine $(0.1 \mathrm{mg} / \mathrm{kg}$ subcutaneously) before surgery in which a proximal colonic anastomosis is performed with seven interrupted extramucosal sutures (Prolene ${ }^{\circledR}$ 8-0; Ethicon, Johnson \& Johnson, Somerville, New Jersey, USA), providing a leakage rate of 33.3 per cent. During surgery, the colon and caecum are kept moist with sterile saline. The body temperature of the mice is held constantly at $37^{\circ} \mathrm{C}$ using a heat pad. The muscle layer and skin are closed using interrupted sutures (Vicryl ${ }^{\circ}$ 4-0 and Monocryl ${ }^{\circledR}$ Plus 4-0 respectively, both from Ethicon). After surgery, the mice receive $0.5 \mathrm{ml}$ saline subcutaneously as fluid therapy. All surgical procedures were performed by a single investigator who was blinded for genotype at the time of surgery.

The main outcome after surgery was macroscopic evaluation of the anastomosis, evaluated as follows: no AL; small abscess, less than $0.5 \mathrm{~cm}^{3}$; large abscess, $0.5 \mathrm{~cm}^{3}$ or more; or faecal peritonitis.

\section{Tissue and blood sampling}

Histological assessment was performed on anastomotic tissue. To conserve the mucous layer, the anastomosis was dissected en bloc, leaving the sutures intact, and fixed in Carnoy solution (60 per cent methanol, 30 per cent chloroform, 10 per cent glacial acetic acid). Unaffected proximal and distal colonic tissues were obtained in the same manner, serving as control tissue. Mesenteric lymph nodes (MLNs) and spleen were snap-frozen in liquid nitrogen for further analysis. Blood samples were taken by intracardial puncture at time of animal killing, collected in EDTA vacuum tubes (BD Vacutainer $\AA$; BD, Stockholm, Sweden) and kept on ice. Blood samples were centrifuged at 4000 r.p.m., at $4^{\circ} \mathrm{C}$ for 15 min. Plasma was stored immediately in aliquots at $-80^{\circ} \mathrm{C}$ until analysis.

\section{Mucus thickness measurements}

To evaluate whether mucus was present at the anastomotic site of control animals, measurements of mucus were performed as described previously ${ }^{28}$, with some modifications. Briefly, unflushed proximal colon was opened longitudinally after 
removal of the mesenteric border. The tissue was pinned down on a silica gel plate with the luminal side facing upwards. Pellet material was gently removed and the mucus was visualized by adding charcoal particles on top of the mucus. The thickness of the mucous layer was then determined by measuring the distance between the mucous surface and the epithelial surface by a micropipette viewed through a stereomicroscope (Leica MZ12; Leica Microsystems, Heerbrugg, Switzerland). Mucus was measured directly at the anastomotic site. Unaffected tissue was used for control measurements. The tissue was submerged in oxygenated Kreb's solution at all times. As Muc2 $2^{-/-}$mice do not have a functional mucous layer, this could not be measured in these animals ${ }^{29}$.

\section{Histological analysis}

Tissue samples were embedded in paraffin and cut in $4-\mu \mathrm{m}$ sections. To evaluate the morphology of cells and glycoproteins in the colonic mucous layer, standard haematoxylin and eosin, and periodic acid-Schiff-Alcian Blue (PAS-AB) staining was performed. Specimens were scored based on inflammation, granulocyte infiltration, fibroblast activity and neoangiogenesis, according to the Ehrlich and Hunt numerical scale (0-4) as modified by Phillips et al. ${ }^{30}$.

Immunohistochemistry for Ki-67 (proliferation), cleaved caspase 3 (CC-3) (apoptosis) and Muc2 (to confirm the phenotype of the animals) was performed. In short, slides were deparaffinized in xylene and rehydrated in graded ethanol to distilled water. Antigen retrieval was performed using target retrieval solution (Dako, Glostrup, Denmark) at $95^{\circ} \mathrm{C}$ for $20 \mathrm{~min}$. Non-specific antibody binding was blocked using 5 per cent fetal calf serum in phosphate-buffered saline (PBS) (Ki-67) or 1 per cent bovine serum albumin (Asp175). Sections were then incubated with specific primary antibodies (Ki-67 clone Sp6; Thermo Fisher Scientific, Life Technologies, Bleiswijk, The Netherlands; CC-3 Asp175, Cell Signalling Technology, Leiden, The Netherlands; or anti-MUC2C3 (provided by the Mucin biology group)9). After washing, swine antirabbit IgG biotin labelled (Dako, Glostrup, Denmark) for Ki-67 and CC-3 stainings, and fluorophore-conjugated CY3 antirabbit antibody (Life Technologies) for Muc2 staining were used as second antibodies. Nuclei were stained using (nickel) 3,3'-diaminobenzidine (DAB) or 4',6-diamidino-2phenylindole (DAPI). All staining was evaluated by two independent observers using microscopy; they were blinded for the genotype of the mice. Ki-67 expression was analysed using ImmunoRatio software ${ }^{31}$, which calculates the percentage of positively stained nuclei (DAB) in relation to the other nuclei (haematoxylin). All CC-3 positive cells in one tissue sample were counted and comparisons were made between control and anastomotic tissue. 


\section{ELISA for intestinal fatty acid binding protein (I-FABP)}

Intestinal fatty acid-binding protein (I-FABP) is a plasma marker for enterocyte damage, as these small cytosolic proteins are present in mature enterocytes at the tip of the villus and released into the circulation following loss of enterocyte membrane integrity ${ }^{32}$. Patients who developed AL after colorectal surgery were shown to have raised I-FABP plasma levels before the operation ${ }^{33}$. I-FABP was measured in plasma using a commercially available sandwich enzyme immunoassay (lower detection limit $0.061 \mathrm{ng} / \mathrm{ml}$; Cloud-Clone, Houston, Texas, USA) according to manufacturer's protocol.

\section{Effect of PGE2 supplementation}

Inhibition of COX has been shown to increase the risk of $\mathrm{AL}$, an effect suggested to be $\mathrm{PGE}_{2}$-mediated ${ }^{27}$. To investigate whether $\mathrm{PGE}_{2}$ has a positive influence on anastomotic healing, mice received either vehicle (PBS) or $100 \mu \mathrm{g} /$ bodyweight of 16,16-dimethyl $\mathrm{PGE}_{2}$ (dmPGE 2 ), a stable $\mathrm{PGE}_{2}$ analogue (Cayman Chemical, Ann Arbor, Michigan, USA), by twice-daily intraperitoneal injection. Both interventions started 1 day before surgery and continued until postoperative day (POD) 3, after which all mice were killed.

\section{qPCR for bacterial translocation}

Total DNA was extracted from snap-frozen MLN and spleen tissue using a combination of sodium dodecyl sulphate and proteolytic digestion with bead beating and phenol/chloroform DNA purification, as previously described ${ }^{34}$. Bead beating was performed using Lysing Matrix E tubes in a FastPrep-24 ${ }^{\mathrm{TM}}$ instrument (MP Biomedicals, Thermo Fisher Scientific, Göteborg, Sweden). DNA was precipitated using $0.2 \mathrm{~mol} / \mathrm{l}$ sodium chloride and isopropanol, then resuspended in $100 \mu \mathrm{L}$ Tris-EDTA buffer and quantified using a NanoDrop ${ }^{\mathrm{TM}}$ ND-1000 spectrophotometer (Thermo Fisher Scientific, Göteborg, Sweden). DNA extractions from tubes containing no tissue served as contamination controls. Purified and quantified 16S DNA from Escherichia coli was used as a standard. Initially, bacterial ribosomal DNA (rDNA) was enriched from host DNA by limited-cycle (LC) PCR amplification of $16 \mathrm{~S}$ rDNA using the universal 16S rDNA primer pair $27 \mathrm{~F}$ (AGAGTTTGATCMTGGCTCAG) and 1492r (CGGTTACCTTGTTACGACTT). Briefly, 50$\mu$ PCR mixtures containing 480 ng DNA, $0.2 \mu \mathrm{mol} / \mathrm{l}$ primers and HotStarTaq Plus Mastermix (Qiagen, Sollentuna, Sweden) were subjected to $5 \mathrm{~min}$ at $95^{\circ} \mathrm{C}$ and 16 cycles of $94^{\circ} \mathrm{C}$ for $1 \mathrm{~min}, 55^{\circ} \mathrm{C}$ for $1 \mathrm{~min}$ and $72^{\circ} \mathrm{C}$ for $1.5 \mathrm{~min}$, followed by $10 \mathrm{~min}$ at $72^{\circ} \mathrm{C}$. Sample, standard and control LC-PCRs were then compared by qPCR using $16 \mathrm{~S}$ rDNA primer pair 926F (AAACTCAAAKGAATTGACGG) and 1062R (CTCACRRCACGAGCTGAC). In brief, 20- $\mu$ l qPCR mixtures were prepared using $2 \mu \mathrm{l}$ LC-PCR mixture, $0.3 \mu \mathrm{mol} / \mathrm{l}$ primers and SSoFast ${ }^{\mathrm{TM}}$ EvaGreen ${ }^{\circledR}$ Supermix (Bio-Rad, 
Sundbyberg, Sweden) and subjected to $95^{\circ} \mathrm{C}$ for $5 \mathrm{~min}$, then 30 cycles of $95^{\circ} \mathrm{C}$ for $15 \mathrm{~s}, 61.5^{\circ} \mathrm{C}$ for $15 \mathrm{~s}$ and $72^{\circ} \mathrm{C}$ for $20 \mathrm{~s}$, with EvaGreen ${ }^{\circledR}$ fluorescence measured at the final step of each cycle. qPCR thermocycling and fluorescence measurement was performed on a CFX96 $6^{\mathrm{TM}}$ instrument (Bio-Rad). Sample amplification curves were compared with contamination control curves, and samples were considered 16Spositive if they amplified before controls. Sample $16 \mathrm{~S}$ was quantified using a standard curve generated from standard qPCR reactions.

\section{Statistical analyses}

Statistical analysis was performed using GraphPad Prism ${ }^{\circledR} 5.0$ for Mac (GraphPad Software, San Diego, California, USA). Normality was tested using the KolmogorovSmirnov test. All continuous variables are presented as mean(s.d.) and compared using Student's $t$ test, the Mann-Whitney $U$ test, ANOVA or the Kruskal-Wallis test as appropriate. Dichotomous variables were compared using the $\chi^{2}$ test.

\section{Results}

\section{AL rate is significantly higher in Muc2 deficient mice}

All mice $(n=44)$ completed follow-up of 3 days. After killing, the abdomen was reopened and the anastomotic site evaluated. Evaluation showed a wide range from normal healing/no AL to small and large abscesses, and even faecal peritonitis (Figure 5.1A-C). Muc2 ${ }^{-/-}$mice had AL more frequently (score greater than 2) compared with control animals (20 of 22 versus 7 of 22 respectively; $P<0.001$ ) (Figure 5.2A). Three animals in the Muc2-/- group suffered from faecal peritonitis, whereas none of the control animals showed any sign of peritonitis. Further, both large ( 8 versus 1 in the control group) and small ( 9 versus 6 respectively) abscesses were found more frequently in Muc2-/- mice. Bodyweight was similar at baseline in the two experimental groups, and remained so over time (Figure 5.2B). A significant decrease in welfare score was observed in Muc2 $2^{--}$mice from POD 1, with maximum effect at POD $3(P<0.001)$ (Figure 5.2C). 

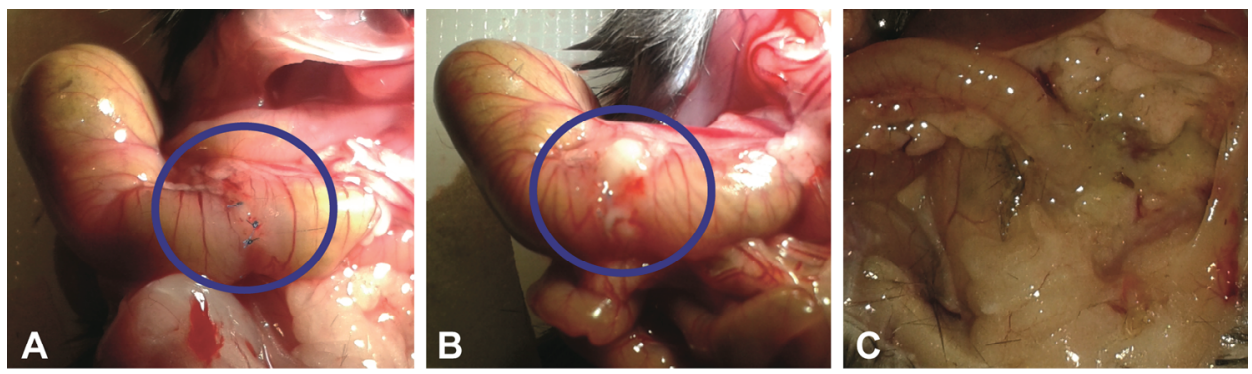

Figure 5.1 Evaluation of the anastomosis: A) no anastomotic leakage, B) abscess (either small, less than $0.5 \mathrm{~cm} 3$, or large, $0.5 \mathrm{~cm} 3$ or more), C) faecal peritonitis
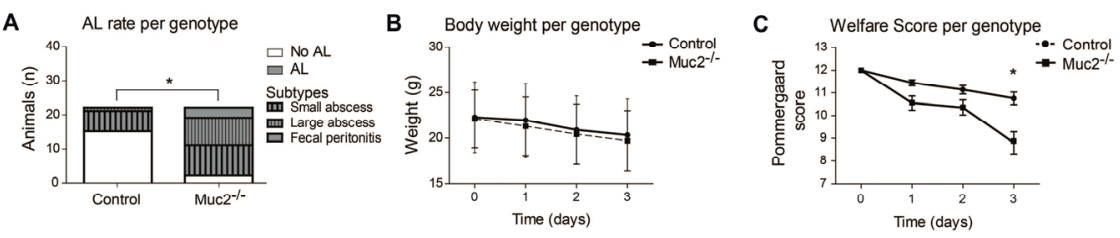

Figure 5.2 A) Muc2-/- mice showed more frequent anastomotic leakage (AL) (score greater than 2; 20 of 22 mice) compared with control animals ( 7 of 22) $* P<0.001$. B) Bodyweight was similar at baseline in the two experimental groups and did not differ over time. C) A significant decrease in Pommergaard welfare score was seen in Muc2-/- mice on day 3 (8.8(1.93) versus $10.76(1.30)$ in control mice; ${ }^{*} P<0.001,2$-way ANOVA with Bonferroni post hoc test (time)).

\section{Mucus is present at the anastomotic site of control animals}

The mucous layer was visualized by the addition of charcoal and its thickness was measured by means of a glass capillary attached to micrometer, observed via a stereomicroscope (Figure 5.3A). A mucous layer was observed in $\mathrm{Muc2}^{+}$animals, at the site of anastomosis as well as in the proximal and distal colonic tissues (Figure 5.3B). In the proximal colon, the mean mucous layer thickness was greatest between folds $(238(17) \mu \mathrm{m})$ and lowest on top of the folds $(71(25) \mu \mathrm{m})$. Mean thickness of the mucous layer at the anastomotic site was $119(65) \mu \mathrm{m}$. In the control group, no significant difference was found in mucous layer thickness between heterozygous $\left(\mathrm{Muc2}^{+/-}\right)$and WT $\left(\mathrm{Muc}^{+/+}\right)$mice, or between mice that had $\mathrm{AL}$ and those that did not. As Muc2-deficient mice lack the structural component of intestinal mucus and therefore lack a defined mucous layer, measurement of the mucous layer in $\mathrm{Muc2}^{-/-}$mice was not feasible. 


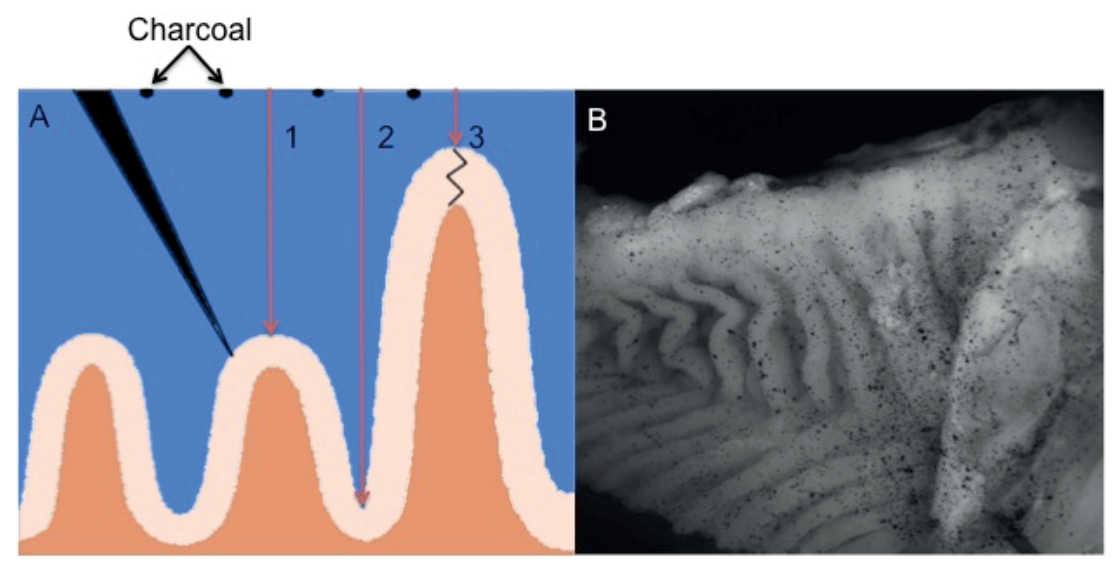

Figure 5.3 Adding charcoal on the sample enables mucus thickness measurements. Mucus thickness is defined as the distance between the charcoal particles and the tissue and was measured on top of colonic folds, in between folds and at the anastomotic site (A). A mucus layer was observed in Muc2+ animals, at the site of anastomosis as well as in the proximal and distal colon control tissues (B).

\section{Histological analysis showed more inflammation in Muc2 deficient mice}

Muc2-/- mice showed a tendency for more leucocyte infiltration and less collagen deposition and neoangiogenesis at killing on POD 3 compared with $\mathrm{Muc2}^{+}$animals (Figure 5.4B). In addition, continuation of the intestinal tissue was evident in the case of normal anastomotic healing, whereas AL caused disruption or dehiscence (Figure 5.4B). There was no difference in proliferation determined by Ki-67 staining between Muc2 ${ }^{-/-}$and control mice at the serosal tissue of the anastomosis or in the enterocytes (Figure 5.4C,D). CC-3 staining showed no differences in apoptosis between Muc2 ${ }^{-/-}$and control mice (Figure 5.4E,F).

A normal inner mucous layer, as shown by PAS-AB staining, was clearly observed in the Muc2 $2^{+}$control mice, but was absent in Muc2-/- mice (Figure 5.5A). In addition, goblet cells in Muc2-/- mice showed only PAS-positive staining, whereas those from control animals also had positive $A B$ staining. Immunohistochemistry revealed the presence of Muc2-positive goblet cells in control animals, but in Muc2-/- mice there was no expression of Muc2, indicating no Muc2-positive goblet cells (Figure 5.5B). 

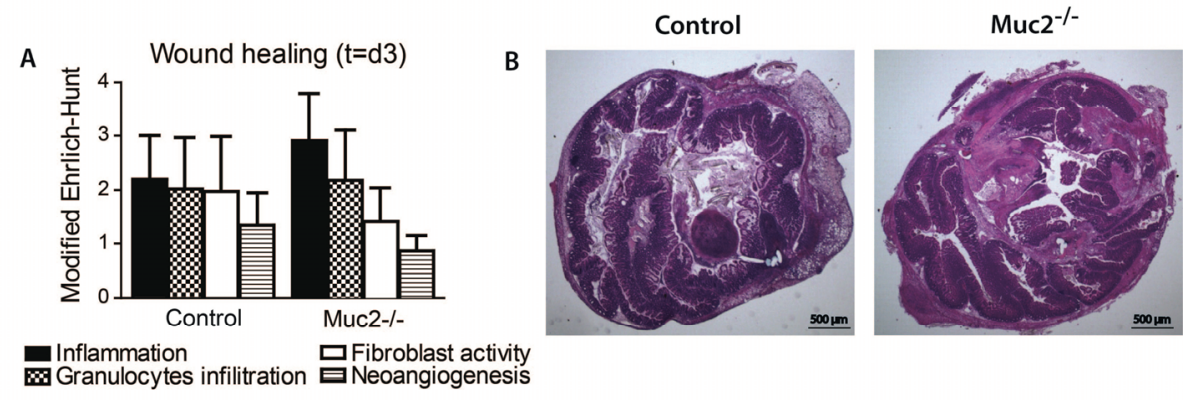

两Granulocytes infilitration
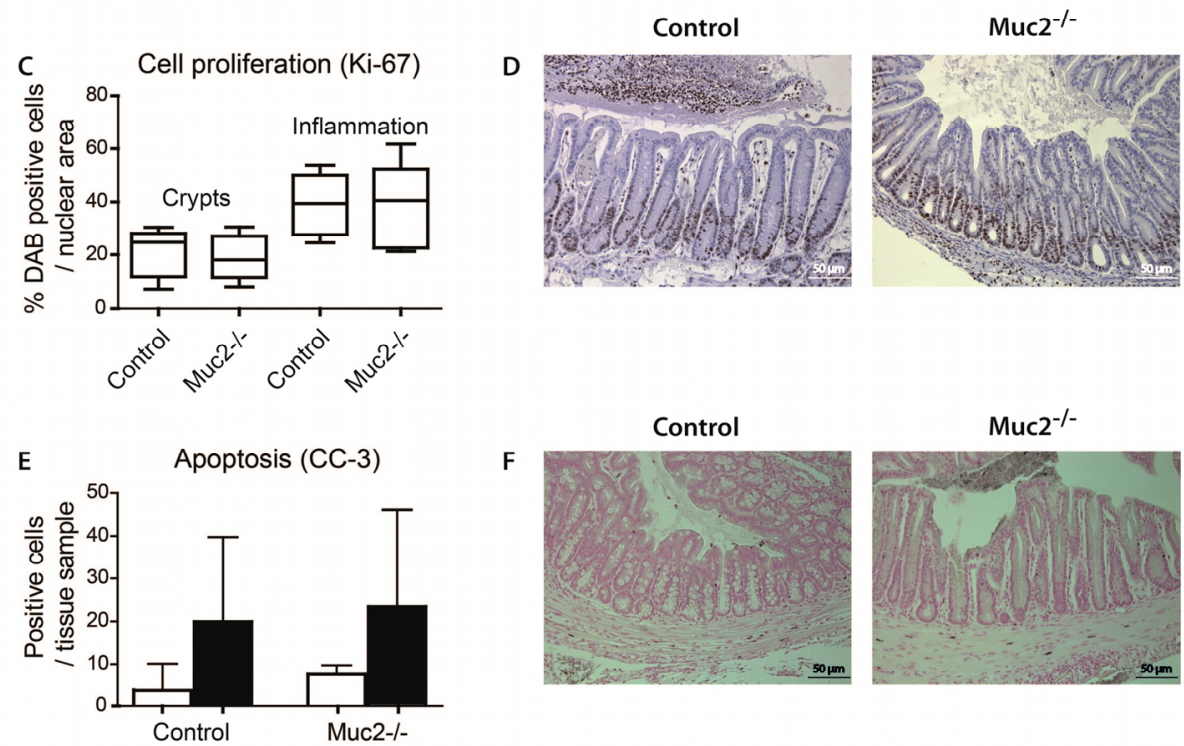

Figure 5.4 A) Inflammatory parameters (mean(s.d.) scored on day 3 after surgery did not differ significantly between experimental groups. B) In Muc2+ animals with normal anastomotic healing there was continuation of the intestinal tissue, whereas anastomotic leakage in Muc2-/- mice caused disruption or dehiscence (arrows) (haematoxylin and eosin stain). $\mathrm{C}+\mathrm{D})$ There was no difference in proliferation measured by Ki-67 staining between Muc2-1and control mice. E+F) 3,3'-Diaminobenzidine (DAB) staining for cleaved caspase (CC) 3 indicated no difference in apoptosis (arrows) between Muc2-/- and control mice. 

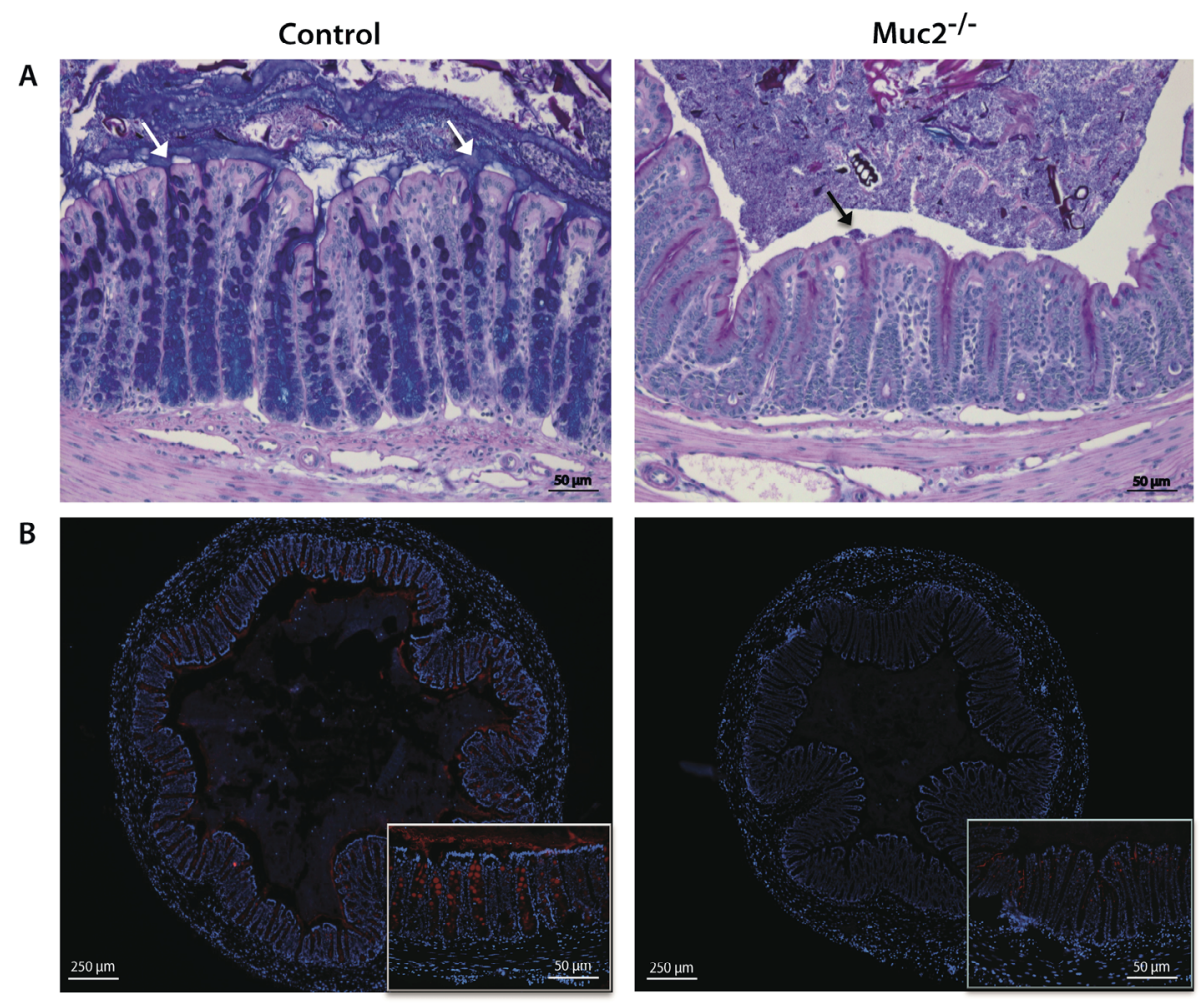

Figure $5.5 \quad A+B)$ A normal inner mucous layer, as shown by periodic acid-Schiff-Alcian Blue (PAS-AB) staining, was clearly observed in the $\mathrm{Muc2}^{+}$control mice (arrows), but was absent in Muc2-/- animals. C+D) Immunohistochemistry revealed the presence of Muc2-positive goblet cells in control animals, but in Muc2-/- mice there was no expression of Muc2, indicating no Muc2-positive goblet cells. The minimal staining found in Muc2-/- is attributed to slight cross-reactivity of the anti-MUC2C3 antiserum with the mouse Fcgbp protein (Fc fragment of IgG-binding protein).

\section{Epithelial cells were damaged in Muc2 deficient mice}

When enterocytes are damaged they leak one of their main cytosolic proteins, IFABP. Hence, measurement of I-FABP in plasma gives an estimate of intestinal tissue damage. Muc2-/- mice showed significantly higher plasma levels of I-FABP compared with control animals (3.88(2.63) versus 1.82(1.83)ng/ml respectively; $\mathrm{P}=0.011$ ) (Figure 5.6A), indicating greater enterocyte damage in the Muc2 ${ }^{-/-}$mice.

\section{$\mathrm{PGE}_{2}$ administration did not reduce anastomosis leakage in this model}

dmPGE2 was administered 1 day before surgery and on every postoperative day until the animals were killed (day 3). With the protocol and doses used, no 
reduction in the rate of $\mathrm{AL}$ was observed in Muc2-/- animals (10 of 11 animals for both vehicle and PGE2). The same was true for control animals (AL in 5 of 11 for vehicle versus 2 of 11 for PGE2; $P=0.362$ ) (Figure 5.6B). Within the control group, no difference was found in $\mathrm{AL}$ rate between heterozygous (Muc2 ${ }^{+/-}$) and WT $\left(\mathrm{Muc}^{+/+}{ }^{+}\right.$mice.

A

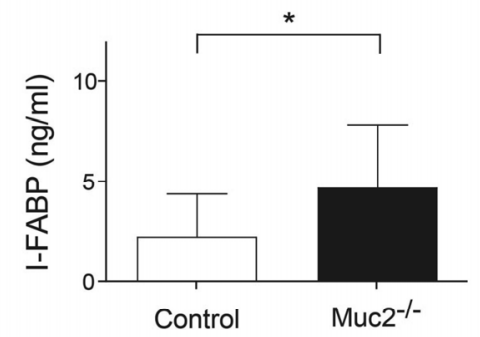

C

$$
\text { Bacterial translocation }
$$
mesenteric lymph node

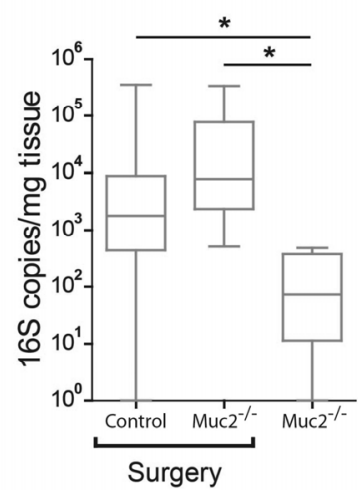

B

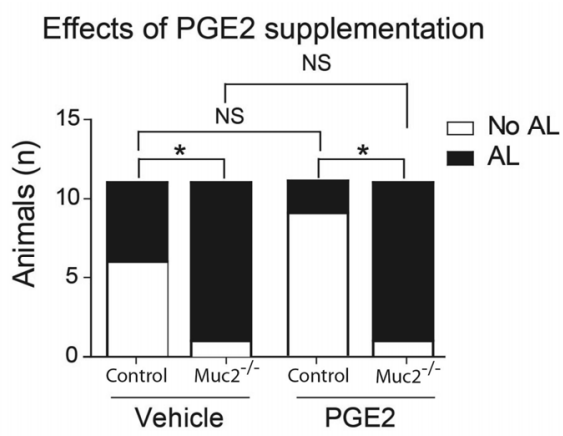

D Bacterial translocation spleen

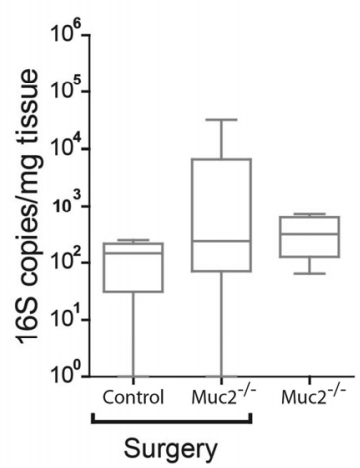

Figure 5.6 A) The concentration of intestinal fatty acid-binding protein (I-FABP) was significantly increased in Muc2-/- mice compared with that in control animals (mean(s.d.) $3.88(2.63)$ versus $1.82(1.83) \mathrm{ng} / \mathrm{ml}$ respectively), indicating more epithelial damage. ${ }^{*} P=0.011$ (Student's $\mathrm{t}$ test). B) Following prostaglandin (PG) E2 supplementation, there was no reduction in the anastomotic leakage (AL) rate for Muc2-/- mice (10 of 11 for both vehicle and PGE2 ), and the reduction in the AL rate in control animals was not significant $(P=0.362$, Student's t test per experiment as well as 2-way ANOVA with post hoc testing ). ${ }^{*} P<0.001$. C) Bacterial translocation to the mesenteric lymph node was present in both control and Muc2 $2^{--}$animals that underwent surgery, but not in Muc2 $2^{--}$mice that had no operation. ${ }^{*} P<0.050$ (Kruskal-Wallis test). Box-and-whisker plots represent median and range. D) No differences were found between experimental groups in bacterial translocation to the spleen. Box-and-whisker plots represent median and range. 


\section{Bacterial translocation in control and Muc2 deficient mice that underwent surgery}

Bacterial 16S rDNA was quantified from MLN and spleen tissue from control and Muc2-/- mice that underwent surgery, and from Muc2-/- mice that had no surgical intervention. The MLN 16S load in both groups of mice having surgery was higher than that in the group that did not, indicating that the surgical procedure did cause increased bacterial translocation to the intestinal lymphatics (Figure 5.6C). No significant difference was detected between control and Muc2-/- mice, although the median 16S load was 4.4 times lower in control mice compared with Muc2-/mice. Spleen load was similar in the three groups, with approximately $10016 \mathrm{~S}$ rDNA copies per mg tissue detected in most animals (Figure 5.6D). However, three of 11 mice in the Muc2-/- surgery group had more than $10416 \mathrm{~S}$ copies/mg, indicating that more bacteria had penetrated the systemic tissue in a subgroup of these animals. Thus, the surgical procedure increased translocation of bacteria from the intestine, and Muc2 deficiency resulted in a tendency towards higher systemic bacterial load.

\section{Discussion}

The incidence of AL has remained high over the years, despite extensive research, the introduction of new surgical techniques and the construction of a diverting stoma in high-risk patients ${ }^{35}$. Many risk factors are being investigated, but the first line of defence in the gastrointestinal tract, the mucous layer, has been largely overlooked.

Mice deficient in the Muc2 gene (Muc2-/- mice) lack a functional mucous layer, and were more prone to develop AL than matched Muc2+/- and Muc2+/+ controls. Control animals had a leakage rate of 32 per cent (7 of 22 animals), in line with the model of Reisinger and colleagues ${ }^{27}$. In addition, Muc2-/- mice had lower welfare scores than control animals despite co-housing and matching for age and sex where possible. It should be noted that $\mathrm{Muc2}^{-/-}$mice develop spontaneous colitis, although this often remains asymptomatic in the animal facility ${ }^{36}$. The effects observed in $\mathrm{Muc}^{-/-}$mice are therefore ascribed to the development of AL rather than to the colitis phenotype. Weight loss did not differ significantly between Muc2-/- and control mice. However, the mice were killed only 3 days after surgery, and it is known that the difference in weight loss between animals with and those without leakage does not become evident until after 3 days ${ }^{25}$. At this time animals without AL start to gain weight, whereas mice with AL start to gain weight only after POD $5^{25}$. Not only did the Muc2-/- mice show more clinical symptoms of AL than control mice, they also had significantly higher I-FABP levels, indicating 
enterocyte damage and more bacterial translocation. The damaged epithelia and increased translocation could indicate that the mucous layer is important for reducing the number of bacteria that reach the vulnerable anastomosis site, and hence is important in the healing process. In line with this, there is accumulating evidence ${ }^{37,38}$ that bacteria are involved in the pathogenesis of AL.

It was hypothesized that supplementation with $\mathrm{dmPGE} 2$ would reduce the $\mathrm{AL}$ rate by increasing mucous secretion. However, dmPGE2 did not reduce the AL rate in either Muc2-/- or control mice. It has been suggested that dmPGE2 can prevent AL when COX-2 activity is decreased, induced either by drug treatment (COX inhibitors) or by knocking out the COX-2 gene ${ }^{27}$. In the present study, no difference in AL rate was observed following PGE2 administration, despite using a stable analogue of PGE2 in the same dosage that had an effect in a Cox2-deficient mouse model. Perhaps PGE2 is effective only when there is a severe deficiency of PGE2, which is not the case in Muc2-/- mice. A higher dose of dmPGE2 induces adverse reactions in mice, with dose- and time-dependent PGE2-induced diarrhoea ${ }^{39}$. In the present study, no diarrhoea was observed, indicating that the PGE2 dose used was safe.

In the present study only anastomoses in the proximal colon, and not in other parts of the gastrointestinal tract, were investigated. Interestingly, Yauw and coworkers ${ }^{40}$ recently showed that diclofenac, a commonly used NSAID, caused AL in the ileum and proximal colon, but not in the distal colon. Furthermore, more recently, Rutegård et al. ${ }^{41}$ found no increased risk of AL with NSAID use in patients undergoing anterior resection for rectal cancer. Previous associations between AL and NSAIDs were derived from pooled data of colorectal surgery, not solely rectal resections. Additionally, it has been shown by ex vivo analysis of the mucous layer that PGE2 caused a significantly increased mucous secretion in the small intestine and less in the proximal colon, and none in the distal colon ${ }^{19}$. Moreover, in humans, membrane protein profiling has demonstrated distinctive regional differences in the colon ${ }^{42}$. These different observations suggest that the healing-promoting effect of PGE2 might differ along the gastrointestinal tract, and that this might also be the case for the healing-promoting effect of a functional mucous layer suggested here for the proximal colon.

Potentially interesting factors that can influence the colonic mucous layer are certain bacteria or SCFAs. A frequent colonizer of the human colonic mucous layer is Akkermansia muciniphila, a mucin-degrading bacterium that is suggested to increase the thickness of the inner mucous layer ${ }^{18}$. The presence of A. muciniphila correlated with increased Muc2 gene expression, suggesting that A. muciniphila can enhance its own nutrient-rich environment ${ }^{43}$. Therefore, it can be hypothesized 
that supplementation with A. muciniphila or other bacteria before gastrointestinal surgery may boost mucous secretion, which can support anastomotic healing.

SCFAs from bacteria in the distal colon are important as the primary energy source for colonic epithelial cells, but also stimulate mucous secretion 16 . One of the most abundant SCFAs in the colon, butyrate, can strengthen distal anastomoses when given in enema form ${ }^{44}$, perhaps through stimulated mucous secretion 16. Thus, commensal bacteria in the outer mucous layer of the distal colon may help to prevent AL in animal models by producing SCFAs. Furthermore, mucins have been suggested to have a trophic effect, as they are not readily digested and can be considered an important nutrient source for many intestinal organisms. Colonic mucus also contains trefoil factor (TFF) 3, a molecule known to have a trophic effect in the colon45. Mucus acts as a diffusion barrier, thereby hindering molecules such as TFF-3 from diffusing out quickly into the lumen, and increasing the local epithelial concentration. The colonic mucus and its main component MUC2 can thus be envisaged to have many effects that might be important for anastomotic healing.

When inflammation and cell proliferation were studied with immunohistochemistry, no differences were observed between the two experimental groups. This might be explained by the fact that mice were killed on day 3 . It is known from other experiments ${ }^{26,27}$ that day 3 is early in the intestinal healing process and that potential differences become evident in later phases of healing. However, POD 3 was chosen for killing in the present study in order to prevent animal suffering.

Results from animal experiments cannot be translated directly to the human setting. Paradoxical to the observation that the thick mucous layer in the distal colon may be protective is that distal tumour location in patients is a known risk factor for the development of AL. For this reason, a diverting stoma is often constructed in clinical practice ${ }^{3,46}$. Although animal findings should be interpreted with caution, they can lead to new insights and provide possible interventions for the human setting. One important lesson is that the controlled ischaemia necessary in surgery can be maintained for some time (up to $1 \mathrm{~h}$ ) and that the mucous layer recovers quickly after this time ${ }^{13}$. Owing to the slow biosynthesis of mucins, a second period of ischaemia should be avoided 47 .

The mucous layer of the gastrointestinal tract has been largely overlooked in research on $\mathrm{AL}$, despite its function as a primary defence mechanism. In this study, mucus has been shown to play an essential role in normal anastomotic healing, and is probably also crucial in other types of gastrointestinal healing. Further research on anastomotic healing should focus on positively influencing the mucous layer, to promote better postoperative recovery. 


\section{References}

1 Bakker IS, Grossmann I, Henneman D, Havenga K, Wiggers T. Risk factors for anastomotic leakage and leak-related mortality after colonic cancer surgery in a nationwide audit. Br J Surg 2014;101: 424-432.

2 Nachiappan S, Askari A, Malietzis G, Giacometti M, White I, Jenkins JT et al. The impact of anastomotic leak and its treatment on cancer recurrence and survival following elective colorectal cancer resection. World J Surg 2015;39:1052-1058.

3 Kingham TP, Pachter HL. Colonic anastomotic leak: risk factors, diagnosis, and treatment. J Am Coll Surg 2009;208:269-278.

4 Telem DA, Chin EH, Nguyen SQ, Divino CM. Risk factors for anastomotic leak following colorectal surgery: a case-control study. Arch Surg 2010;145:371-376.

5 Pommergaard HC, Gessler B, Burcharth J. Preoperative risk factors for anastomotic leakage after resection for colorectal cancer: a systematic review and meta-analysis. Colorectal Dis 2014;16: $662-671$.

6 Gessler B, Bock D, Pommergaard H-C, Burcharth J, Rosenberg J, Angenete E. Risk factors for anastomotic dehiscence in colon cancer surgery - a population-based registry study. Int J Colorectal Dis 2016;31:895-902.

7 Shogan BD, Carlisle EM, Alverdy JC, Umanskiy K. Do we really know why colorectal anastomoses leak? J Gastrointest Surg 2013;17:1698-1707.

8. Bosmans JW, Moossdorff M, Al-Taher M, van Beek L, Derikx JP, Bouvy ND. International consensus statement regarding the use of animal models for research on anastomoses in the lower gastrointestinal tract. Int J Colorectal Dis 2016;31:1021-1030.

9 Johansson ME, Phillipson M, Petersson J, Velcich A, Holm L, Hansson GC. The inner of the two Muc2 mucin-dependent mucus layers in colon is devoid of bacteria. Proc Natl Acad Sci U S A 2008;105: 15 064-15 069.

10 Johansson ME, Sjövall H, Hansson GC. The gastrointestinal mucus system in health and disease. Nat Rev Gastroenterol Hepatol 2013;10:352-361.

11 Ambort D, Johansson MEV, Gustafsson JK, Nilsson HE, Ermund A, Johansson BR et al. Calcium and pH-dependent packing and release of the gel-forming MUC2 mucin. Proc Natl Acad Sci U S A 2012; 109:5645-5650.

12 Johansson ME. Fast renewal of the distal colonic mucus layers by the surface goblet cells as measured by in vivo labeling of mucin glycoproteins. PLoS One 2012;7:e41009.

13 Grootjans J, Hundscheid IH, Lenaerts K, Boonen B, Renes IB, Verheyen FK, et al. Ischaemia-induced mucus barrier loss and bacterial penetration are rapidly counteracted by increased goblet cell secretory activity in human and rat colon. Gut 2013;62:250-258.

14 Birchenough GM, Johansson ME, Gustafsson JK, Bergström JH, Hansson GC. New developments in goblet cell mucus secretion and function. Mucosal Immunol 2015;8:712-719.

15 Smith AC, Podolsky DK. Colonic mucin glycoproteins in health and disease. Clin Gastroenterol 1986;15:815-837.

16 Barcelo A, Claustre J, Moro F, Chayvialle JA, Cuber JC, Plaisancié P. Mucin secretion is modulated by luminal factors in the isolated vascularly perfused rat colon. Gut 2000;46:218-224.

17 Coconnier MH, Dlissi E, Robard M, Laboisse CL, Gaillard JL, Servin AL. Listeria monocytogenes stimulates mucus exocytosis in cultured human polarized mucosecreting intestinal cells through action of listeriolysin O. Infect Immun 1998;66:3673-3681.

18 Everard A, Belzer C, Geurts L, Ouwerkerk JP, Druart C, Bindels LB et al. Cross-talk between Akkermansia muciniphila and intestinal epithelium controls diet-induced obesity. Proc Natl Acad Sci U S A 2013;110:9066-9907.

19 Ermund A, Schütte A, Johansson MEV, Gustafsson JK, Hansson GC. Studies of mucus in mouse stomach, small intestine, and colon. I. Gastrointestinal mucus layers have different properties depending on location as well as over the Peyer's patches. Am J Physiol Gastrointest Liver Physiol 2013;305:G341-G347. 
20 Vane JR, Botting RM. Anti-inflammatory drugs and their mechanism of action. Inflamm Res 1998; 47(Suppl 2):S78-S87.

21 Holte K, Andersen J, Jakobsen DH, Kehlet H. Cyclo-oxygenase 2 inhibitors and the risk of anastomotic leakage after fast-track colonic surgery. Br J Surg 2009;96:650-654.

22 Rushfeldt CF, Sveinbjørnsson B, Søreide K, Vonen B. Risk of anastomotic leakage with use of NSAIDs after gastrointestinal surgery. Int J Colorectal Dis 2011;26:1501-1509.

23 de Hingh IH, van Goor H, de Man BM, Lomme RM, Bleichrodt RP, Hendriks T. Selective cyclooxygenase 2 inhibition affects ileal but not colonic anastomotic healing in the early postoperative period. Br J Surg 2006;93:489-497.

24 Kilkenny C, Browne WJ, Cuthill IC, Emerson M, Altman DG. Improving bioscience research reporting: the ARRIVE guidelines for reporting animal research. PLoS Biol 2010;8:e1000412.

25 Pommergaard H-C, Achiam MP, Rosenberg J. Colon anastomotic leakage: improving the mouse model. Surg Today 2013;44:933-999.

26 Komen N, van der Wal H-C, Ditzel M, Kleinrensink G-J, Jeekel H, Lange JF. Colorectal anastomotic leakage: a new experimental model. J Surg Res 2009;155:7-12.

27 Reisinger KW, Schellekens DH, Bosmans JW, Boonen B, Hulsewé KW, Sastrowijoto P et al. Cyclooxygenase-2 is essential for colorectal anastomotic healing. Ann Surg 2016; [Epub ahead of print].

28 Gustafsson JK, Ermund A, Johansson ME, Schütte A, Hansson GC, Sjövall H. An ex vivo method for studying mucus formation, properties, and thickness in human colonic biopsies and mouse small and large intestinal explants. Am J Physiol Gastrointest Liver Physiol 2012;302:G430-G438.

29 Johansson ME, Gustafsson JK, Holmén-Larsson J, Jabbar KS, Xia L, Xu H et al. Bacteria penetrate the normally impenetrable inner colon mucus layer in both murine colitis models and patients with ulcerative colitis. Gut 2014;63:281-291.

30 Phillips JD, Kim CS, Fonkalsrud EW, Zeng H, Dindar H. Effects of chronic corticosteroids and vitamin A on the healing of intestinal anastomoses. Am J Surg 1992;163:71-77.

31 Tuominen VJ, Ruotoistenmaki S, Viitanen A. ImmunoRatio: a publicly available web application for quantitative image analysis of estrogen receptor (ER), progesterone receptor (PR), and Ki-67. Breast Cancer Res 2010;12:R56.

32 Schellekens DH, Grootjans J, Dello SA, van Bijnen AA, van Dam RM, Dejong CH et al. Plasma intestinal fatty acid-binding protein levels correlate with morphologic epithelial intestinal damage in a human translational ischemia-reperfusion model. J Clin Gastroenterol 2014;48:253-260.

33 Reisinger KW, Poeze M, Hulsewé KW, van Acker BA, van Bijnen AA, Hoofwijk AG et al. Accurate prediction of anastomotic leakage after colorectal surgery using plasma markers for intestinal damage and inflammation. J Am Coll Surg 2014;219:744-751.

34 Zoetendal EG, Heilig HG, Klaassens ES, Booijink CC, Kleerebezem M, Smidt H et al. Isolation of DNA from bacterial samples of the human gastrointestinal tract. Nat Protoc 2006;1:870-873.

35 Daams F, Luyer M, Lange JF. Colorectal anastomotic leakage: aspects of prevention, detection and treatment. World J Gastroenterol 2013;19:2293-2297.

36 Wenzel UA, Magnusson MK, Rydström A, Jonstrand C, Hengst J, Johansson ME et al. Spontaneous colitis in Muc2-deficient mice reflects clinical and cellular features of active ulcerative colitis. PLoS One 2014;9:e100217.

37 Shogan BD, Belogortseva N, Luong PM, Zaborin A, Lax S, Bethel C et al. Collagen degradation and MMP9 activation by Enterococcus faecalis contribute to intestinal anastomotic leak. Sci Transl Med 2015;7:286ra68.

38 Olivas AD, Shogan BD, Valuckaite V, Zaborin A, Belogortseva N, Musch M et al. Intestinal tissues induce an SNP mutation in Pseudomonas aeruginosa that enhances its virulence: possible role in anastomotic leak. PLoS One 2012;7:e44326.

39 Riviere PJ, Farmer SC, Burks TF, Porreca F. Prostaglandin E2-induced diarrhea in mice: importance of colonic secretion. J Pharmacol Exp Ther 1991;256:547-552.

40 Yauw ST, Lomme RM, van der Vijver RJ, Hendriks T, van Laarhoven KJ, van Goor H. Diclofenac causes anastomotic leakage in the proximal colon but not in the distal colon of the rat. Am J Surg 2015;210:382-388. 
41 Rutegård M, Westermark S, Kverneng Hultberg D, Haapamäki M, Matthiessen P, Rutegård J. Nonsteroidal anti-inflammatory drug use and risk of anastomotic leakage after anterior resection: a protocol-based study. Dig Surg 2016;33:129-135.

42 van der Post S, Hansson GC. Membrane protein profiling of human colon reveals distinct regional differences. Mol Cell Proteomics 2014;13:2277-2287.

43 Ganesh BP, Klopfleisch R, Loh G, Blaut M. Commensal Akkermansia muciniphila exacerbates gut inflammation in Salmonella typhimurium-infected gnotobiotic mice. J Am Coll Surg 2015;220: 186-194.

44 Bloemen JG, Schreinemacher MH, de Bruine AP, Buurman WA, Bouvy ND, Dejong CH. Butyrate enemas improve intestinal anastomotic strength in a rat model. Dis Colon Rectum 2010;53: 1069-1075.

45 Judd LM, Chalinor HV, Walduck A, Pavlic DI, Däbritz J, Dubeykovskaya Z et al. TFF2 deficiency exacerbates weight loss and alters immune cell and cytokine profiles in DSS colitis, and this cannot be rescued by wild-type bone marrow. Am J Physiol Gastrointest Liver Physiol 2015;308:G12-G24.

46 Shiomi A, Ito M, Maeda K, Kinugasa Y, Ota M, Yamaue $\mathrm{H}$ et al. Effects of a diverting stoma on symptomatic anastomotic leakage after low anterior resection for rectal cancer: a propensity score matching analysis of 1014 consecutive patients. J Am Coll Surg 2015;220:186-194.

47 Johansson MEV, Hansson GC. The goblet cell: a key player in ischaemia-reperfusion injury. Gut 2013;62:188-189. 


\section{Supplementary information according to ARRIVE guidelines}

- Muc2-/- mice backcrossed on C57BL/6 background between 8-16 weeks of age were used. Muc2-/- mice on the C57BL/6 background were bred as Muc2 ${ }^{+/-} \times$ Muc2 ${ }^{+/-}$at the Experimental Biomedicine animal facility at the University of Gothenburg and offspring were genotyped.

- Mice were kept under standard conditions and were provided with food and water ad libitum. Mice were housed together with their littermates, with a maximum of 6 companions per cage. The general health of mice was monitored several times per week for signs of inflammation and animals were weighed once per week. During the experiment animals were weighed daily and scored for discomfort twice daily.

- Scoring was performed according Pommergaard et al:

\begin{tabular}{llc}
\hline Parameter & Grading & Score \\
\hline Activity & Normal/medium/low & $2 / 1 / 0$ \\
Fur & Smooth/fluffy/erect & $2 / 1 / 0$ \\
Eyes & Clean and open/clean and closed/dirty and closed & \\
Able to stand straight & Yes/no & $1 / 0$ \\
Posture & Normal/modestly curied/fully curled up & $2 / 1 / 0$ \\
Position on feet & Normal/high & $1 / 0$ \\
Solitary & Yes/no & $0 / 1$ \\
Shivering & Yes/no & $0 / 1$ \\
\hline
\end{tabular}

Scoring was done by one researcher who was blinded for treatment at the time of scoring, while the other researcher performed preparation of the PGE2 or placebo syringes for peritoneal injection.

- Protocols were approved by the government animal ethics committee and institutional animal use and care guidelines were followed (permit 63-2014).

- $\quad$ Mice were sacrificed by intracardial puncture after isoflurane anaesthesia using an airstream of $2 \mathrm{l} / \mathrm{min}$ and $3.5 \%$ isoflurane.

- A sample size calculcation was conducted prior to the experiment based on an expected reduction in anastomotic leakage of $20 \%$. To detect a relevant difference in leakage reduction of $20 \%(\delta)$ between the different experimental groups with a variance of $\pm 16 \%(\sigma)$, the number of animals per group should be: (poweranalysis $\alpha: 0.05, \pi: 0.8,2$ sided testing):

- $\mathrm{n}=2(\mathrm{z} \alpha / 2-\mathrm{z} \pi) 2 *(\sigma / \delta) 2$ (Sachs)

- $\mathrm{n}=2(1.96+0.84)^{2 *}(16 / 20)^{2}$

- $\mathrm{n}=15.7 * 0.64=10.03 \approx 11 /$ group

- In total there were 52 mice used in this study. We used 11 per experimental group, and 4 groups (knockout vs control mice, with and without dmPGE2 supplementation). In addition, 8 mice were sacrificed to obtain control tissue (MLN, spleen) for bacterial translocation analysis. 
- Animals were matched for age and gender where possible, making it impossible to use randomisation procedures. 


\section{Part III}

Reducing anastomotic leakage rate:

glues and enema intervention 


\title{
CHAPTER 6
}

\author{
Comparison of three different \\ application routes of butyrate
}

to improve colonic anastomotic strength in rats

J.W.A.M. Bosmans, A.C.H.M. Jongen, B.T.C. Boonen, S. van Rijn, F. Scognamiglio, L. Stucchi, M.J.J. Gijbels, E. Marsich, N.D. Bouvy Int J Colorectal disease, 2016 


\section{Abstract}

\section{Introduction}

Despite extensive research, anastomotic leakage (AL) remains one of the most dreaded complications after colorectal surgery. Since butyrate enemas are known to enhance anastomotic healing, several administration routes have been explored in this study.

\section{Methods}

Three intraluminal approaches involving butyrate were investigated: 1) butyrin-elucidating patch; 2) a single injection of hyaluronan-butyrate (HA-But) prior to construction of the proximal anastomosis; and 3) rectal hyaluronan-butyrate (HA-But) enemas designed for distal anastomoses. The main outcome was AL and secondary outcomes were bursting pressure, histological analysis of the anastomosis, zymography to detect MMP activity and qPCR for gene expression of MMP2, MMP9, MUC2 and TFF3.

\section{Results}

Neither the patches nor the injections led to a reduction of AL in experiment 1 and 2. In experiment 3, a significant reduction of AL was accomplished with the (HA-But) enema compared to the control group together with a higher bursting pressure. Histological analysis detected only an increased inflammation in experiment 2 in the hyaluronan injection group compared to the control group. No other differences were found regarding wound healing. Zymography identified a decreased pro-enzyme of MMP9 when HA-But was administered as a rectal enema. qPCR did not show any significant differences between groups in any experiment.

\section{Conclusions}

Butyrate enemas are effective in the enhancement of colonic anastomosis. Enhanced butyrate-based approaches designed to reduce AL in animal models for both proximal and distal anastomoses were not more effective than butyrate enemas alone. Further research should focus on how exogenous butyrate can improve anastomotic healing after gastrointestinal surgery. 


\section{Introduction}

Despite extensive research, anastomotic leakage (AL) remains one of the most dreaded complications after colorectal surgery. It still results in high morbidity and mortality, and causes prolonged hospital stay and high health care costs ${ }^{1-3}$. Many studies have been performed regarding this subject, all aiming at improvement of anastomotic healing, and thereby preventing leakage. Since the incidence of anastomotic leakage has not declined over the years, the need for a solution continues to exist ${ }^{4,5}$.

Previous animal studies have shown that rectal administration of butyrate - one of the predominant short chain fatty acids (SCFAs) -, results in a higher bursting pressure in colonic anastomoses, thereby claiming an enhanced strength which may prevent anastomotic leakage ${ }^{6-8}$.

These positive results did not change current practice, nor have human studies been reported in which patients received postoperative butyrate enemas to enhance anastomotic strength. This is not surprising, when you consider that clinicians are not keen on enemas directly after surgery, especially when a fragile rectal anastomosis is involved. Water-soluble contrast enemas are often used to determine the presence of colorectal AL, mostly after rectum resection. However, these enemas are never given in the very early postoperative phase (<postoperative day 5) due to the potential risk of complications ${ }^{5}$. Another disadvantage of the use of enemas is the low compliance rate and the short and discontinuous exposure of butyrate to the colonic mucosa.

A recent study however showed that butyrate has remarkable antiinflammatory and regenerative effects improving the repair of intestinal mucosa in rats with colitis ${ }^{9}$. Butyrate stimulates re-epithelisation and may influence collagen lysis by reducing matrix metalloproteinase (MMP) release ${ }^{10}$. Butyrate is a product of the fermentation process by bacteria that occurs mainly in the distal colon. Because it serves as the most important energy source for colonocytes ${ }^{11}$, our hypothesis is that increasing the concentration of butyrate will lead to an enhanced anastomotic healing in the colon. A number of studies indicate that butyrate affects the composition and thickness of the colonic mucus layer through alteration of mucin gene expression ${ }^{12}$ and nutritional deficiency of the colonic epithelium and that butyrate also can act as a signalling molecule through specific G-protein coupled receptors that are involved in the immune surveillance of the colonic mucosa towards microbial activity ${ }^{13}$. More recently, it has been shown that mice 
with an insufficient mucus layer on the colonic mucosa (Muc2-/-) suffered more often from anastomotic leakage compared to control mice ${ }^{14}$.

Furthermore, animal studies showed that butyrate enemas have a significant positive effect on the left colonic anastomoses, but this effect was weaker on the right side of the colon ${ }^{8}$. Obviously, not the same volume will reach the proximal anastomotic site compared to the distal anastomosis, which may explain these findings. Another explanation might be that the butyrate-producing bacteria mainly reside in the mucus layer at the distal part of the colon, that the proximal colon does not respond to a higher amount of butyrate, simply because the proximal enterocytes are not familiar to having that energy source available and are not able to use it. To increase the beneficial effect of butyrate, we added hyaluronan (HA), a glycosaminoglycan that is known to promote neovascularization, to enhance the process of scarring, and has beneficial effects on cell proliferation in several tissues $^{15,16}$. Hyaluronan also plays a role in the downregulation of the inflammatory response. Its free-radical scavenging and antioxidant properties and its supposed inhibiting effect on proteinases such as MMPs, seem to be responsible for this downregulation, stabilizing the granulation tissue during the healing process $^{16}$.

In this study, we investigated several ways to improve the proximal anastomotic strength by applying butyrate near this anastomosis: using a patch; injections; and enemas. Our aim was to develop a method that resulted in a lower anastomotic leakage rate and more practical methods that can be applied in daily clinical practice.

\section{Methods}

\section{Animals}

Eighty-four Wistar rats with a body weight of 250-300 gram were used. Animals were housed and cared for at the Central Animal Facility of Maastricht University. All animals were provided ad libitum access to food and water, and were cared for according to local standards. Postoperatively, welfare assessment was performed twice daily using a standardized method and animals were given pain medication in case of discomfort. The experimental protocol complied with the Dutch Animal Experimental Act and was approved by the Animal Experimental Committee of Maastricht University Medical Center. The ARRIVE guidelines for reporting in vivo experiments were followed ${ }^{17}$ (see also Supplementary Data S6.1). 


\section{Study design}

Primary objective of the study was to investigate if anastomotic leakage can be reduced in a leakage model by application of either an intraluminal butyrin-eluting patch, a single-time intraluminal administration of butyrate or a daily HA-But enema. For the proximal anastomoses we used both an intraluminal butyrin-eluting patch that covered the anastomosis and a single-time intraluminal administration of hyaluronan-butyrate (HA-But). Since most research is performed in the distal part of the colon, we also used a left-colon anastomosis model to test the HA-But enemas. This resulted in the following 3 experiments:

Experiment 1: Butyrin eluting patches (20 massa\% tributyrin) versus placebo patches ( $\mathrm{n}=12$ /group).

Experiment 2: HA-But injection ( $5 \%$ HA, 60mM butyrate) versus HA injection ( $5 \% \mathrm{HA})$ versus Saline injection $(0.9 \% \mathrm{NaCl})$ ( $\mathrm{n}=8$ /group).

Experiment 3: $5 \mathrm{~mL}$ of HA-But enema (5\% HA, $60 \mathrm{mM}$ butyrate) versus $5 \mathrm{~mL}$ sodium Butyrate $(60 \mathrm{mM})$ enema versus a control group that received no enemas ( $\mathrm{n}=12$ /group).

Patches were kindly provided by Polyganics (Groningen, the Netherlands) and enemas and injections were produced by Sigea (Trieste, Italy). In all experiments, follow up was 7 days.

\section{Surgical procedure}

All rats received $0.05 \mathrm{mg} / \mathrm{kg}$ buprenorphine as analgesic and were anesthetized using isoflurane. To acquire access to the abdominal cavity, a $5 \mathrm{~cm}$ craniocaudal midline incision of the skin and abdominal musculature was made in all experiments. The cecum was then identified and moved outside of the peritoneal cavity and onto sterile gauzes that were hydrated with sterile saline solution to prevent dehydration. For proximal anastomoses: the colon was transected two centimeters distal from the cecum and an end-to-end anastomosis was created using 4 interrupted polypropylene 6/0 sutures (Prolene, Ethicon, Johnson \& Johnson) (Exp 1 \& 2, see Supplementary Data S6.1). In Experiment 3, the anastomosis was performed in similar fashion but at 4 centimeters ab ani. After performing the anastomosis, the intestines were repositioned and the abdomen was closed in two layers, a running suture for the muscle layer (Vicryl 4-0, Ethicon, Inc) and interrupted sutures for the skin (Monocryl 4-0, Ethicon, Inc).

\section{Macroscopic endpoints}

Anastomotic leakage was defined as 1) no anastomotic leakage, 2) small abscess at the anastomotic site $\left.<1 \mathrm{~cm}^{3}, 3\right)$ large $\left(>1 \mathrm{~cm}^{3}\right)$ abscess at the anastomotic site or 4$)$ 
complete dehiscence with peritonitis/death due to fecal peritonitis. Adhesions to the anastomotic site were assessed according to van der Ham et al. ${ }^{18}$.

\section{Bursting pressure}

Bursting pressure was measured as previously described ${ }^{19}$. In short, a 5-cm segment of intestine including the anastomosis with and adherent organs was resected en bloc and the part distal of the anastomosis was clamped. A plastic tube was inserted in the proximal end and ligated with a single polyglactine 4/0 suture (Vicryl, Ethicon). The complete anastomosis was immersed in phosphate buffered saline, while air was infused using a manometer (IDEE, Maastricht University, The Netherlands) and pressure was manually increased by inflating the colon. Bursting pressure was defined as the intraluminal pressure at which air leakage was initially observed from the anastomosis (mBar).

\section{Tissue preparation}

In anesthetized rats, the anastomotic site was dissected (after measuring bursting pressure) with a $0.5 \mathrm{~cm}$ margin at each site of the anastomosis. Tissue samples were divided in three equal pieces, one for qPCR, one for zymography purposes and one for immunohistochemistry purposes. This latter one was cut in longitudinal direction and tissue was stretched and pinned onto a cork layer in order to secure a straight anastomotic line and improve quality of histological assessment prior to fixation in formalin. Sections were deparaffinized in xylene and rehydrated in graded ethanol to distilled water prior to performing histological staining. The part dedicated for qPCR analysis was snap frozen in liquid nitrogen and stored at $-80^{\circ} \mathrm{C}$ until required for mRNA isolation.

\section{Histology and immunohistochemistry}

Sections were stained with standard hemotoxylin-eosin and Picro Sirius Red using Direct Red 80 and Picric Acid solution (both Sigma-Aldrich, the Netherlands).

An independent, experienced animal pathologist performed blinded histological assessment on the obtained tissue, scoring (from 0-4) histological sections on inflammation, granulocyte influx, fibroblast activity and collagen deposition. Collagen ration was calculated using an in-house software program on pictures obtained with a polarized light microscope (Leica DM5000B, Leica Microsystems, Wetzlar, Germany).

\section{MMP2, pro-MMP9 and MMP9 activity analysis using zymography}

MMP-2 and MMP-9 activity were assessed using gelatin zymography since it increased MMP activity has been implicated in the pathogenesis of $\mathrm{AL}^{20}$. Samples 
were lysed from tissue and loaded on a polyacrylamide gel containing gelatin $(10 \%$ Ready Zymogram Gel, Bio-Rad, the Netherlands). Gel electrophoresis took place at $100 \mathrm{~V}$ during $30 \mathrm{~min}$. After that, the gel was incubated with $2.5 \%$ Triton X-100 in MQ for $30 \mathrm{~min}$ at room temperature. The gel was then placed in developing buffer at $37^{\circ} \mathrm{C}$ overnight. Colouring of the gel took place the following night with PAGE-Blue protein staining solution (Thermo Scientific, the Netherlands). At day 3, the staining was decoloured with MQ, revealing MMP-2 and MMP-9 activity. The gels were scanned using a Bio-Rad GS-800 Densitometer and digitally quantification was done using ImageJ software (ImageJ Software, U. S. National Institutes of Health, Bethesda, Maryland, USA).

\section{MMP2, MMP9, TFF3 and Muc2 expression analysis using qPCR}

The expression of matrix metalloproteinases 2 (MMP2) and 9 (MMP9) as well as Mucin 2 (MUC2) and trefoil factor 3 (TFF3) was determined using qPCR to see if exogenous butyrate had an effect on the expression of these MMPs; the abundant secretory mucin MUC2 as well as its associated trefoil factor. All qPCR-experiments were carried out by a dedicated technician (BB) and are reported as detailed as possible according to the MIQE guidelines ${ }^{21}$. RNA was isolated from the tissue sample using TRI reagent (Sigma-Aldrich, the Netherlands) and treated with RNase-Free DNase Set (Qiagen). The purity of RNA was tested using Nanodrop Spectrophotometer (Thermo Scientific) by 260/280 absorbance (A260/A280) ratios, ranged from 1.89 to 2.02 . Therefore, the purity of the isolated RNA was satisfactory for further studies. RNA of 750ng was converted to DNA with sensifast cDNA Synthese kit (Bioline, London, UK). qPCR was performed on 10ng cDNA with $0,3 \mu \mathrm{M}$ primers in Sensimix SYBR \& Fluorescein Kit (Bioline, London, UK) using a white 384 wells qPCR plate. Primer sequences are listed in Table 6.1. Reference genes included were glyceraldehyde-3-phosphate dehydrogenase (GAPDH) and Bactin. Reactions were run on the LightCycler480 (Roche). PCR conditions used were $10 \mathrm{~min}$ at $95^{\circ} \mathrm{C}$, followed by 40 amplification cycles of $15 \mathrm{~s}$ at $95^{\circ}-60^{\circ} \mathrm{C}-72^{\circ} \mathrm{C}$ followed by a melting curve to validate the amplifications. The cycle threshold (Ct) value of each reference gene was obtained from q-PCR analysis. The obtained Ct values were applied to evaluate the expression of the genes of interest. The mean $\mathrm{Ct}$ value of the reference genes ranged from 16 to 23 cycles, with HA-injection having the highest transcript levels in MMP9 and butyrin patch was associated with having the lowest transcript levels in TTF3. For analysis purposes, the level of MMP2 expression of the saline group was set at 1 and used as a control.

\section{Statistical analyses}

Statistical analysis was performed using Prism 5.0 for Mac (Graphpad software, San Diego, CA). Normality was tested using Kolmogorov-Smirnov. All continuous 
variables are presented as mean and standard error of the mean (SEM) and compared using students t-test or Mann Whitney $U$ when appropriate. Dichotomous variables were compared using the Chi-square test.

Table 6.1 Primer sequences used for qPCR experiments.

\begin{tabular}{ll}
\hline r-MMP9-F1 & AGCCGACGTCACTGTAACTG \\
r-MMP9-R1 & AACAGGCTGTACCCTTGGTC \\
r-MMP2-F1 & ACAACAGCTGTACCACCGAG \\
r-MMP2-R1 & GGACATAGCAGTCTCTGGGC \\
R-MUC-F1 & CGAAGTGAAGAGTGAGCACG \\
R-MUC-R1 & GATCCGGGTGGTATTCAGCA \\
R-Tff-F1 & GGCCTATCTCCAAGCCAATGT \\
R-Tff-R1 & TGCAGAGGTTTGAAGCACCA \\
GAPDH FW & GGAAGCTCACTGGCATGGC \\
GAPDH RV & CCTGCTTCACCACCTTCTTG \\
Beta-actin FW & GACAGGATGCAGAAGGAGATTACTG \\
Beta-actin RV & CCACCGATCCACACAGAGTACTT \\
\hline
\end{tabular}

\section{Results}

\section{Macroscopical results}

In experiment 1 , two rats in the butyrin patch group died prior to completing follow-up due to faecal peritonitis. All animals in experiment 2 completed the 7-day follow up. In experiment 3, three rats (1 in the HA-But group, 2 in the But group) were euthanized because they had reached humane endpoints, due to faecal peritonitis. An overview of the types of anastomotic leakage within each experimental group can be found in Figure 6.1A. Adhesions occurred in every experiment and there were no significant differences between experiments (proximal versus distal anastomoses) nor within each experiment (Figure 6.1B). It became evident that animals receiving a patch (either butyrin eluting or placebo) had lower welfare scores and significant more weight loss compared to animals receiving enemas (Figure 6.1C), possibly due to an extended bowel proximal to the location of the patch upon sacrifice. 
A

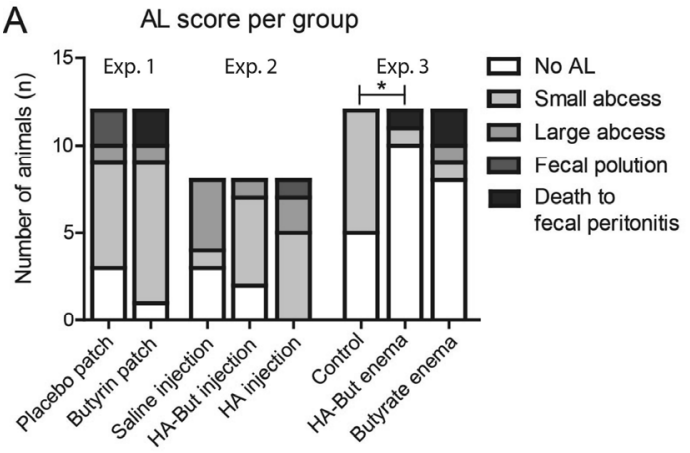

C

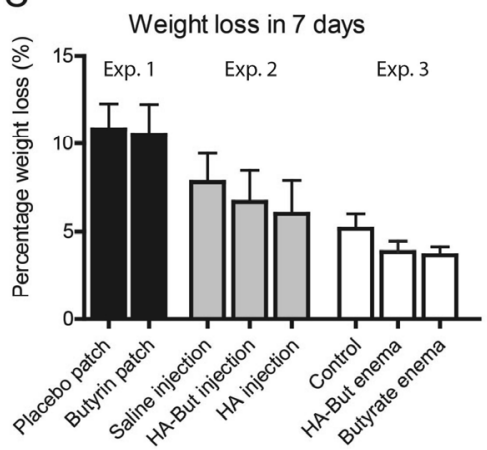

B Adhesion score
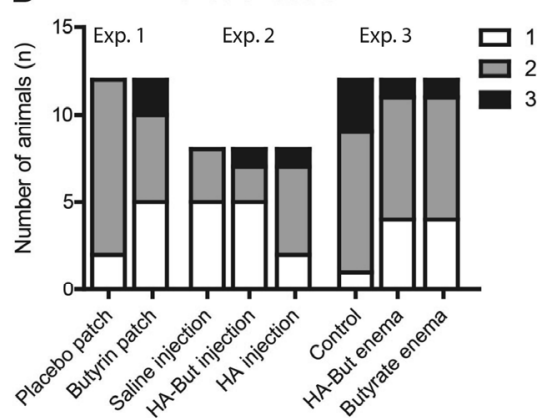

D

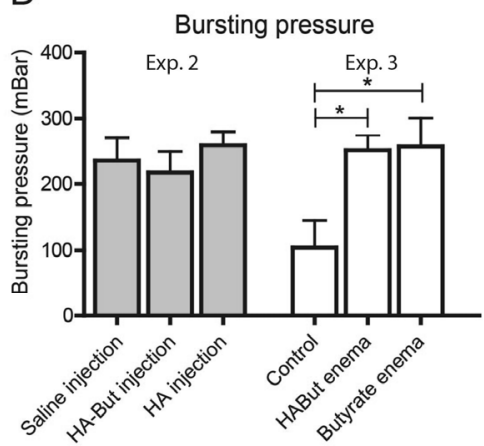

Figure 6.1 Butyrate-enriched enemas reduced AL in Exp. 3, no other butyrate-based intervention caused a decrease in AL rates (A). Adhesion scores did not differ significantly between groups in Exp. 1,2 or 3 (B). Weight loss was most prominent in Exp. 1 where placement of the patches caused a sudden change in calibre of the colon; Animals receiving enemas had the lowest percentage of weight loss but also received a different type of anastomosis compared to animals in Exp. 1 and 2 (distal vs proximal respectively) (C). The administration of butyrate enemas caused a significant higher bursting pressure of the anastomosis in Exp. 3 compared to the control group. No differences were found in Exp. 2 and in Exp. 1 no BP was measured due to porous, distended intestines.

\section{Bursting pressure}

Bursting pressure (BP) was not measured during experiment 1 because of evident extended intestines in the majority of the rats at the place where the patches were placed. This abrupt change in luminal calibre made the dilated bowel very porous, disabling accurate BP measurements. In experiment 2, BP was measured and compared to a historical control group since the control group from experiment 3 received a distal anastomosis. No differences were found in experiment 2 between saline injections, HA injections and HA-But injections. In experiment 3 however, a 
significant difference could be found between the control group (104.1 \pm 40.8$)$ and HA-But (250.7 \pm 23.1$)$ and But enemas $(256.4 \pm 44.4, P<0.05)$.

\section{Histology and collagen ratio}

Inflammatory parameters were scored as a surrogate marker of anastomotic healing, as indicated appropriate by a recent Delphi consensus method 22 . Histological analysis (Figure 6.2) only detected an increased inflammation in experiment 2 in the hyaluronan injection group (2.18 \pm 0.22$)$ compared to the control group $(3.27 \pm 0.27, P<0.05)$. No other differences were found regarding wound healing. Picro Sirius red staining, which depicts the level of collagen maturity, of the anastomotic region showed comparable percentages of collagen for all groups (Table 6.2).

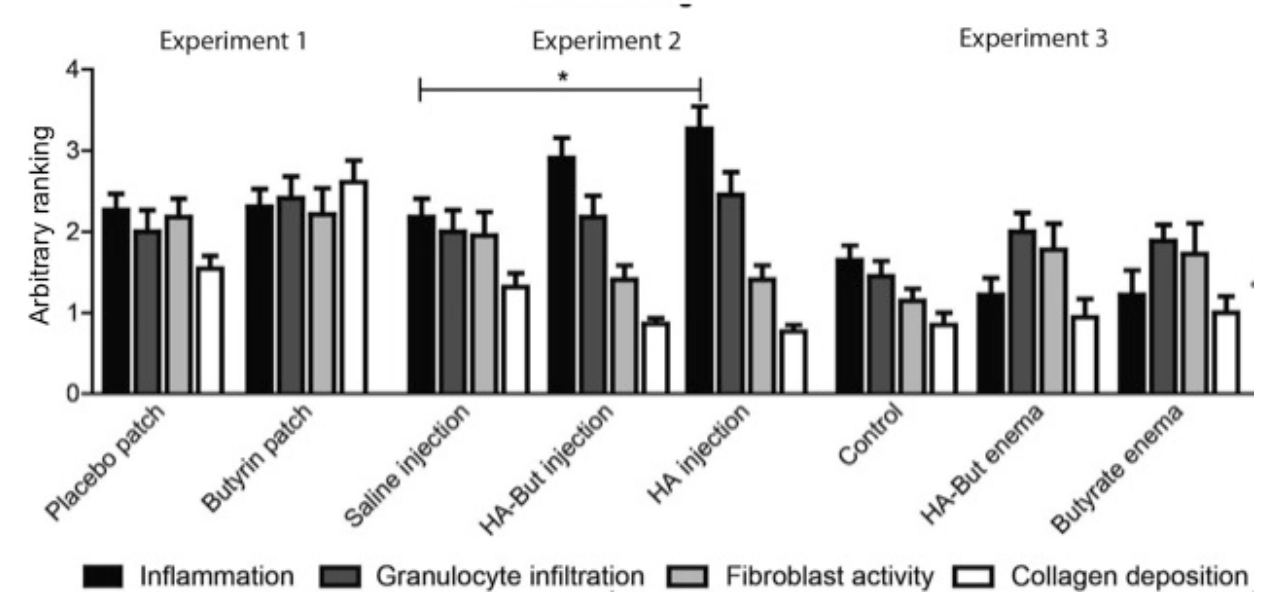

Figure 6.2 Histological analysis detected only an increased inflammation in experiment 2 in the hyaluronan injection group $(2.18 \pm 0.22)$ compared to the control group $(3.27 \pm 0.27$, $P<0.05)$. No other differences were found regarding wound healing. Values are mean ranking (S.E.M.).

\section{Pro-MMP9, MMP2 and MMP9 activity measured by zymography}

Zymography revealed no differences between MMP2 and MMP9 activity within each experiment between control and intervention groups (Figure 6.3). There was relatively large variability within the samples of each group as can be seen by the error bars/whiskers in the different graphs. In experiment 3, the addition of hyaluronan to the butyrate enema did decrease the activity of pro-MMP9 (40.6 \pm 8.2) compared to the butyrate enema alone (102.6 \pm 26.9$)$ and the control group $(114 \pm 20.9, P=0.01)$. 
Table 6.2 Total collagen and mature/immature collagen ratios.

\begin{tabular}{|c|c|c|c|c|}
\hline Experiment 1 & Butyrin patch & Placebo patch & & $P$-value \\
\hline Collagen percentage $(\%)$ & $32.25( \pm 2.69)$ & $28.64( \pm 2.00)$ & & 0.239 \\
\hline Red/green ratio & $464.83( \pm 278.36)$ & $361.42( \pm 207.96)$ & & 0.861 \\
\hline Experiment 2 & Saline injection & HA-But injection & HA injection & $P$-value \\
\hline Collagen percentage $(\%)$ & $53.22( \pm 2.04)$ & $45.29( \pm 4.51)$ & $46.50( \pm 4.83)$ & 0.361 \\
\hline Red/green ratio & $66.89( \pm 16.76)$ & $39.66( \pm 7.88)$ & $95.64( \pm 44.43)$ & 0.340 \\
\hline Experiment 3 & Control & HA-But enema & Butyrate enema & $P$-value \\
\hline Collagen percentage $(\%)$ & $42.84( \pm 4.75)$ & $35.57( \pm 4.23)$ & $35.93( \pm 2.12)$ & 0.343 \\
\hline Red/green ratio & $321.50( \pm 71.60)$ & $282.00( \pm 154.85)$ & $176.54( \pm 46.35)$ & 0.355 \\
\hline
\end{tabular}

The relative collagen area was quantified as the percentage of total tissue surface. Maturity of collagen was estimated by calculating the red/green ratio. Red indicates thick, mature collagen. Green indicates thin, immature collagen. In terms of collagen percentage and red/green ratio, no significant differences were found between groups. All measurements in this table are presented as mean $( \pm$ SEM).
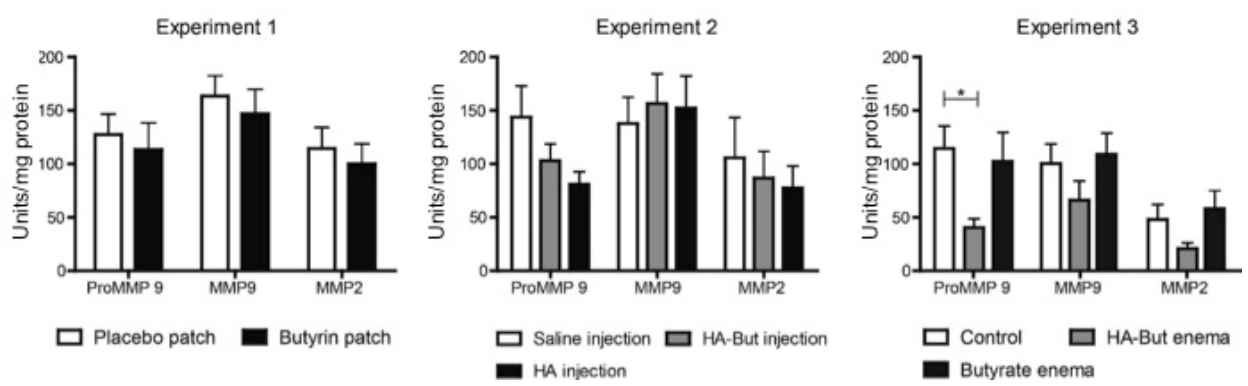

Figure 6.3 Zymography revealed no differences between MMP2 and MMP9 activity within each experiment between control and intervention groups. The addition of hyaluronan to the

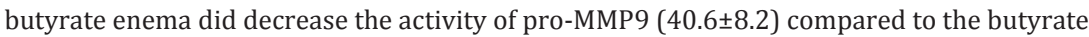
enema alone $(102.6 \pm 26.9)$ and the control group $(114 \pm 20.9)$ in experiment $3(P=0.01)$. Values are given in arbitrary units and data represent mean + S.E.M.

\section{qPCR showed no significant differences in the 3 experiments regarding gene expression}

In experiment 1, a trend was found in the expression of MMP2 between the group that received a butyrin patch $(0.35 \pm 44.4)$ and the placebo patch group $(1.14 \pm$ $0.34, P<0.07$, Figure 6.4). The other tested matrix-metalloproteinase MMP9 also showed a trend between these two groups, $0.39 \pm 0.21$ versus $1.14 \pm 0.34$ respectively, $P<0.08$. No differences were found regarding MUC2 and TFF3.

In experiment 2 and 3 no significant differences were found in gene expression between the intervention and control groups regarding all genes of interest. 

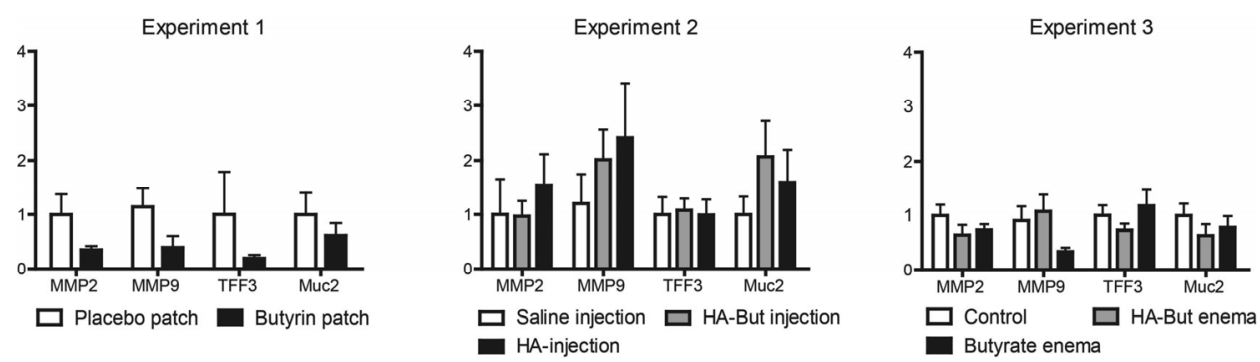

Figure 6.4 Overview of relative gene expression where the level of MMP2 expression of the saline group was set at 1 and used as a control. No significant differences were found in gene expression between the intervention and control groups regarding all genes of interest in the separate experiments.

\section{Discussion}

This study is the first study as far as we know that aims to improve butyrate supplementation in animal models to overcome the reservation of surgeons to optimize their patients with butyrate prior or during gastrointestinal surgery. We investigated different administration routes besides enemas that could enable surgeons to incorporate butyrate in the surgical procedure. Unfortunately, a single dosage of butyrate or a butyrin-eluting patch that we used in this study did not seem promising. Addition of hyaluronan to butyrate did not improve the effect of an enema on the healing of distal anastomoses. Unfortunately, this study did not lead to promising tools to transport butyrate to the proximal anastomotic site, but it did confirmed that butyrate can lead to better anastomotic healing and this should be further investigated in the future.

Butyrate is known to have a critical mediating role in the colonic inflammatory response, for example it can induce the differentiation of colonic regulatory $\mathrm{T}$ cells and thereby ameliorate the development of colitis as shown by Furusawa et al ${ }^{23}$. More recently, it has been shown that exogenous butyrate restoration improved intestinal epithelial cell junctional integrity, decreased apoptosis and mitigated graft-versus-host-disease after allogeneic bone marrow transplant ${ }^{24}$. Especially the restorative effect of butyrate on the epithelial integrity and, consequently, a decreased translocation of luminal contents are of great interest in the field of anastomotic leakage. It was often assumed that butyrate increases collagen synthesis and maturation and that this reduced the risk of anastomotic leakage $\mathrm{e}^{6-8}$, however with these new insights, it can be hypothesized that increased epithelial integrity leads to a lesser amount of (clinically relevant) anastomotic leakage. 
As stated before, enemas seem to be a promising tool to deliver butyrate in the colon, especially in more distal anastomoses, however it remains difficult to reach the same levels of butyrate in proximal anastomoses. In addition, butyrate is metabolized rapidly as soon as it enters the colonocyte via its active transport system. This could be the reason that experiment 2 was not successful; the amount of available butyrate by a single injection was simply not sufficient to cause any effect on the anastomotic site.

It was suggested that a pro-drug of natural butyrate, tributyrin, could overcome the pharmacokinetic drawbacks of natural butyrate as a drug 25 . Since it was very difficult to incorporate butyrate in an eluting patch, butyrin was used instead, making sure that there were high concentrations available at the site of release. Unfortunately the attachment of a patch into the colon caused extension of the bowel proximal to the location of the patch, indicating colonic stenosis. This effect of patches or fleeces has been previously described ${ }^{26}$, however in that study the authors used an external fleece and in experiment 1 the film-like resorbable patches were attached intraluminally. This stenosis effect - clinically translatable to ileus - could be the explanation of why the animals in experiment 1 had lower welfare scores and lower weight compared to the other groups.

Hyaluronan is generally known to be associated with tissue repair and wound healing and its concentration is high in healing tissues ${ }^{27}$. Its biological functions are the result of both its physicochemical properties and its biological interactions, depending on molecular weight ${ }^{28}$. Despite the logical hypothesis that the addition of hyaluronan to butyrate would enhance anastomotic healing, this was not observed in this study, possibly due to a low concentration or not the appropriate molecular weight.

One of the limitations of this study - even if the newly tested approaches were successful - is the translation to the human setting. While butyrate can be administered as a rectal enema, this is rather invasive and not the preferred choice in drug administration. The risk of anastomotic dehiscence in a recent constructed anastomosis remains, which could be an explanation of the fecal peritonitis that was found in the enema groups in experiment 3. However, the very unpleasant odour and taste of butyrate makes oral administration extremely difficult. For this reason, Raso et al have studied a derivative of butyrate, N-(1-carbamoyl-2-phenylethyl) butiramide (FBA) as a successful therapeutic alternative to butyrate, sharing a comparable efficacy, but a better palatability and compliance, unfortunately so far only in a rat model ${ }^{29}$. Other articles that describe oral administration of butyrate were also designed as animal studies ${ }^{30,31}$. Nevertheless, there are a few studies in 
which oral butyrate or its pro-drug was given in humans and was considered safe and well tolerated ${ }^{32}$. The question remains if butyrate can be administered as a local agent at the site of the anastomosis, without potential harmful effects when it enters the systemic circulation. Van Beek et al. have recently demonstrated that splanchnic butyrate release was prevented in patients after colonic butyrate administration, indicating that rectal enemas even with high therapeutic dosage (up to $100 \mathrm{mmol} / \mathrm{l}$ ) of butyrate can be given safely ${ }^{33}$.

Conceivably new approaches may become available as nano-based drug delivery systems for encapsulation and release of drugs are currently being investigated in different fields of medicine ${ }^{34,35}$. Imaginably in the future butyrate tablets become standard perioperative treatment and due to nanotechnology the release of butyrate will only take place at the site of interest, the colonic anastomosis.

In conclusion, butyrate can enhance the colonic anastomosis, making it less prone to lead to leakage. The most effective method thus far is by rectal enemas and the addition of hyaluronan did not ameliorate the effect of butyrate on the AL rate. Further research should be done to identify the optimal way of butyrate delivery to the anastomotic site where it can enhance anastomotic healing. 


\section{References}

1. Snijders HS, Wouters MWJM, van Leersum NJ, Kolfschoten NE, Henneman D, de Vries AC, et al. Meta-analysis of the risk for anastomotic leakage, the postoperative mortality caused by leakage in relation to the overall postoperative mortality. Eur J Surg Oncol. 2012;38:1013-1019.

2. Bakker IS, Grossmann I, Henneman D, Havenga K, Wiggers T. Risk factors for anastomotic leakage and leak-related mortality after colonic cancer surgery in a nationwide audit. Br J Surg. 2014;101:424-432.

3. Nachiappan S, Askari A, Malietzis G, Giacometti M, White I, Jenkins JT, et al. The Impact of Anastomotic Leak and Its Treatment on Cancer Recurrence and Survival Following Elective Colorectal Cancer Resection. World J Surg. 2014;1-7.

4. Morks AN, Havenga K, Ploeg RJ. Can intraluminal devices prevent or reduce colorectal anastomotic leakage: a review. World J Gastroenterol. 2011;17:4461-4469.

5. Daams F, Luyer M, Lange JF. Colorectal anastomotic leakage: aspects of prevention, detection and treatment. World J Gastroenterol. 2013;19:2293-2297.

6. Bloemen JG, Schreinemacher MH, de Bruine AP, Buurman WA, Bouvy ND, Dejong CH. Butyrate enemas improve intestinal anastomotic strength in a rat model. Dis Colon Rectum. 2010;53:10691075.

7. Netta S, Michalopoulos A, Apostolidis S, Paramythiotis D, Papavramidis T, Papadopoulos V, et al. Enhancement of colonic anastomotic strength in rats by short-chain fatty acids. Tech Coloproctol. 2010;14:53-55.

8. Mathew AJ, Wann VC, Abraham DT, Jacob PM, Selvan BS, Ramakrishna BS, et al. The Effect of Butyrate on the Healing of Colonic Anastomoses in Rats. J Invest Surg. 2010;23:101-104.

9. Pacheco RG, Esposito CC, Müller LCM, Castelo-Branco MTL, Quintella LP, Chagas VLA, et al. Use of butyrate or glutamine in enema solution reduces inflammation and fibrosis in experimental diversion colitis. World J Gastroenterol. 2012;18:4278-4287.

10. Knudsen KEB, Serena A, Canibe N, Juntunen KS. New insight into butyrate metabolism. Proc Nutr Soc. 2003;62:81-86.

11. Cook, Sellin. Review article: short chain fatty acids in health and disease. Aliment Pharmacol Ther. 1998;12:499-507.

12. Jung TH, Park JH, Jeon WM. Butyrate modulates bacterial adherence on LS174T human colorectal cells by stimulating mucin secretion and MAPK signaling pathway. Nutr Res Pract. 2015;9:3439.13.

13. Hamer HM, Jonkers D, Venema K, Vanhoutvin S, Troost FJ, Brummer R-J. Review article: the role of butyrate on colonic function. Aliment Pharmacol Ther. 2008;27:104-19.

14. Bosmans JWAM, Jongen ACHM, Birchenough GMH, Nyström EEL, Gijbels MJJ, Derikx JPM, et al. Normal mucus composition is essential in the colonic anastomotic healing in mice [Internet]. uegw.congress-online.com. 2015. Available from: https://uegw.congressonline.com/guest/ID050c7f65a50e5d/AbstractView?ABSID=10275

15. Price RD, Berry MG, Navsaria HA. Hyaluronic acid: the scientific and clinical evidence. J Plast Reconstr Aesthet Surg. 2007;60:1110-1119.

16. Sikkink CJJM. Applications of hyaluronan in abdominal surgery. 2011. Thesis Radboud University Nijmegen, the Netherlands. Available from: http://repository.ubn.ru.nl/bitstream/handle/2066/90827/90827.pdf?sequence=1

17. NC3Rs Reporting Guidelines Working Group. Animal research: reporting in vivo experiments: the ARRIVE guidelines. J Physiol. 2010;588:2519-2521

18. van der Ham AC, Kort WJ, Weijma IM, van den Ingh HF, Jeekel J. Effect of fibrin sealant on the healing colonic anastomosis in the rat. Br J Surg. 1991;78:49-53.

19. Vogels RRM, Bosmans JWAM, van Barneveld KWY, Verdoold V, van Rijn S, Gijbels MJJ, et al. A new poly(1,3-trimethylene carbonate) film provides effective adhesion reduction after major abdominal surgery in a rat model. Surgery. 2015;157:1113-1120. 
20. Ågren MS, Andersen TL, Andersen L, Schiødt CB, Surve V, Andreassen TT, et al. Nonselective matrix metalloproteinase but not tumor necrosis factor- $\alpha$ inhibition effectively preserves the early critical colon anastomotic integrity. Int J Colorectal Dis. 2010;26:329-337.

21. Bustin SA, Benes V, Garson JA, Hellemans J, Huggett J, Kubista M, et al. The MIQE guidelines: minimum information for publication of quantitative real-time PCR experiments. Clin Chem;2009:611-22.

22. Bosmans JWAM, Moossdorff M, Al-Taher M, van Beek L, Derikx JPM, Bouvy ND. International consensus statement regarding the use of animal models for research on anastomoses in the lower gastrointestinal tract. Int J Colorectal Dis. 2016;31:1021-1030.

23. Furusawa Y, Obata Y, Fukuda S, Endo TA, Nakato G, Takahashi D, et al. Commensal microbederived butyrate induces the differentiation of colonic regulatory T cells. Nature. 2013;504:446450 .

24. Mathewson ND, Jenq R, Mathew AV, Koenigsknecht M, Hanash A, Toubai T, et al. Gut microbiomederived metabolites modulate intestinal epithelial cell damage and mitigate graft-versus-host disease. Nat Immunol. 2016;17:505-513.

25. Gaschott T, Steinhilber D, Milovic V, Stein J. Tributyrin, a stable and rapidly absorbed prodrug of butyric acid, enhances antiproliferative effects of dihydroxycholecalciferol in human colon cancer cells. J Nutr. 2001;131:1839-1843.

26. Schreinemacher MH, Bloemen JG, Heijden SJ, Gijbels MJ, Dejong CH, Bouvy ND. Collagen fleeces do not improve colonic anastomotic strength but increase bowel obstructions in an experimental rat model. Int J Colorectal Dis. 2011;26:729-735.

27. Litwiniuk M, Krejner A, Speyrer MS, Gauto AR, Grzela T. Hyaluronic Acid in Inflammation and Tissue Regeneration. Wounds. 2016;28:78-88.

28. Maytin EV. Hyaluronan: More than just a wrinkle filler. Glycobiology. 2016;26:553-559.

29. Mattace Raso G, Simeoli R, Russo R, Iacono A, Santoro A, Paciello O, et al. Effects of sodium butyrate and its synthetic amide derivative on liver inflammation and glucose tolerance in an animal model of steatosis induced by high fat diet. Alisi A, editor. PLoS ONE. 2013;8:e68626.

30. Vieira ELM, Leonel AJ, Sad AP, Beltrão NRM, Costa TF, Ferreira TMR, et al. Oral administration of sodium butyrate attenuates inflammation and mucosal lesion in experimental acute ulcerative colitis. J Nutr Biochem. 2012;23:430-436.

31. Russo R, De Caro C, Avagliano C, Cristiano C, La Rana G, Mattace Raso G, et al. Sodium butyrate and its synthetic amide derivative modulate nociceptive behaviors in mice. Pharmacol Res. 2016;103:279-291.

32. Conley BA, Egorin MJ, Tait N, Rosen DM, Sausville EA, Dover G, et al. Phase I study of the orally administered butyrate prodrug, tributyrin, in patients with solid tumors. Clin Cancer Res. 1998;4:629-634.

33. van der Beek CM, Bloemen JG, van den Broek MA, Lenaerts K, Venema K, Buurman WA, et al. Hepatic Uptake of Rectally Administered Butyrate Prevents an Increase in Systemic Butyrate Concentrations in Humans. Journal of Nutrition. American Society for Nutrition; 2015;145: 2019-2024.

34. Dolati S, Sadreddini S, Rostamzadeh D, Ahmadi M, Jadidi-Niaragh F, Yousefi M. Utilization of nanoparticle technology in rheumatoid arthritis treatment. Biomed Pharmacother. 2016;80:30-41.

35. Fasehee H, Dinarvand R, Ghavamzadeh A, Esfandyari-Manesh M, Moradian H, Faghihi S, et al. Delivery of disulfiram into breast cancer cells using folate-receptor-targeted PLGA-PEG nanoparticles: in vitro and in vivo investigations. J Nanobiotechnology. 2016;14:32. 


\section{Supplemental Data 6.1}

\section{Ethical statement}

- The experimental protocol complied with the Dutch Animal Experimental Act and was approved by the Animal Experimental Committee of Maastricht University Medical Center. Protocols for institutional animal use and care guidelines were followed (permit DEC 2013-101).

\section{Study design}

- In total there were 84 rats used in this study. We used 8 or 12 rats per experimental group, and 2-3 groups per experiment, as is shown in the table below:

\begin{tabular}{lllll}
\hline Experiment & Intervention & Control group & Number of animals & Proximal/Distal colon \\
\hline 1 & Butyrin-eluting & Placebo patch & $12 /$ group & Proximal \\
& patch & & 24 in total & \\
2 & Hyaluronan- & Hyaluronan injection & $8 /$ group & Proximal \\
butyrate injection & and saline injection & 24 in total & \\
3 & Hyaluronan- & Butyrate enema and & $12 /$ group & Distal \\
& butyrate enema & no enema & 36 in total & \\
\hline
\end{tabular}

\section{Experimental procedures}

- $\quad$ For surgical procedure, see manuscript and figure S6.1 below. This was carried out in the rat operating room of the animal facility of Maastricht University under semi-sterile conditions.
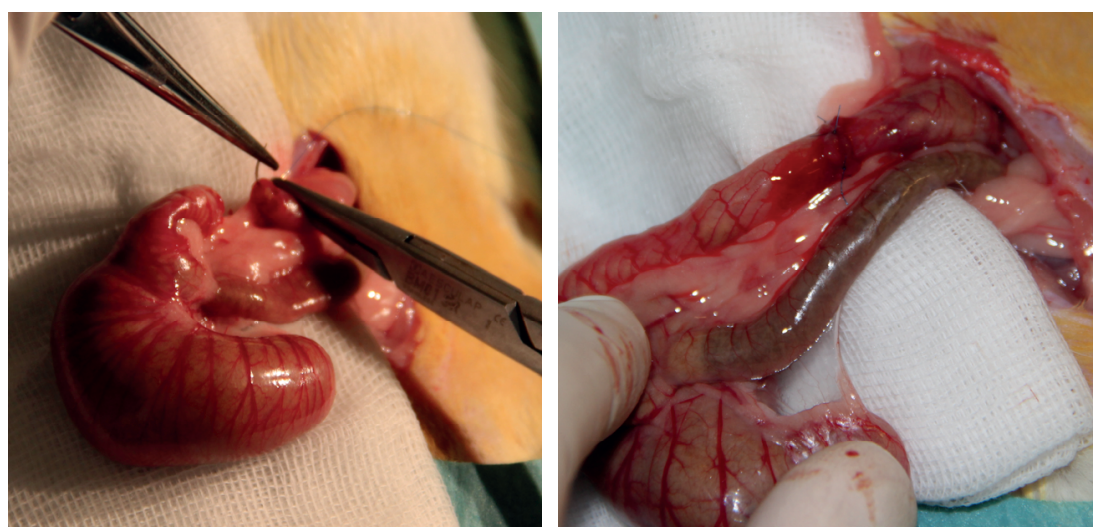

Figure S6.1 Creating the proximal colonic anastomosis after transection (A), constructed using 4 interrupted sutures (B). 


\section{Experimental animals}

- Male Wistar rats (RccHan:WIST) were purchased from Harlan laboratories, the Netherlands with a weight of $275 \mathrm{~g}$. Rats had an average weight of $327 \mathrm{~g}$ on the day of surgery (SD 19.3).

- We have chosen for male rats, since it is known that female estrogens and androgens have an important influence on wound-healing (Ashcroft GS, et al. Estrogen modulates cutaneous wound healing by downregulating macrophage migration inhibitory factor. The Journal of clinical investigation. 2003, 111:1309-1318).

\section{Housing and husbandry}

- An acclimatization period of one week was observed prior to the start of the experiment.

- $\quad$ Rats were kept under standard conditions and were provided with food and water ad libitum. Rats were housed 2 animals per cage. The general health of rats was monitored several times per week for signs of inflammation and animals were weighed once per week. During the experiment animals were weighed daily and scored for discomfort twice daily (every morning and every evening). In case of discomfort, additional pain treatment was administered by giving buprenorphine $0.05 \mathrm{mg} / \mathrm{kg}$ s.c.

- Discomfort was scored using a standard scheme (zie below). Humane endpoints were defined according to Roughan \& Flecknell (Roughan, J. V. \& Flecknell, P. A. Behavioural effects of laparotomy and analgesic effects of ketoprofen and carprofen in rats. Pain 90, 65-74 (2001).

- Humane endpoints are defined as:

- Significant weight loss $>20 \%$

- Fever (temperature is only measured on indication)

- Tachypnoe

- Significant differences in behavior:

- Lethargia

- Twitching: random spasms of the muscles, can be seen when animals are asleep or inactive in huddled up position.

- Walking: unable to stand on four legs, wobbly walk

- Huddled up posture: showing a concave abdominal side. Can be seen when walking/sitting.

○ Signs of severe dehydration

- Severe diarrhea

- Severely inflammed surgical wound

- Cachexia 


\section{Allocating animals to experimental groups}

- Animals were randomized by means of taking a card out of an envelope on which either 1-2-3 was printed. For each animal a card was drawn and the rat received the assigned treatment. We had 3 separate envelopes for each experiment a new envelope (with either 2 or 3 types of card in there).

\section{Numbers analysed}

- All analyses were performed according to an intention-to-treat analysis. However, since there were adverse events and deaths prior to follow-up, for $\mathrm{ABP}$ and histological assessment these animals were not taken into account.

\section{Welfare scoring rat model}

\begin{tabular}{|c|c|c|c|c|}
\hline \multicolumn{5}{|l|}{ Description } \\
\hline & 0 & 1 & 2 & 3 \\
\hline Activity & Normal & Isolated, less active & Inactive & $\begin{array}{l}\text { Somnulent, stupor, } \\
\text { coma, lifeless }\end{array}$ \\
\hline Behavior & Normal & $\begin{array}{l}\text { Back arching, } \\
\text { twitching, shivering } \\
\text { Once } / 10 \text { min }\end{array}$ & $\begin{array}{l}\text { Back arching, twitching, } \\
\text { shivering }\end{array}$ & $\begin{array}{l}\text { Stereotype behavior, } \\
\text { auto mutilation, } \\
\text { aggressive behavior }\end{array}$ \\
\hline Gait & Normal & $\begin{array}{l}\text { Mildly uncoordinated/ } \\
\text { Abnormality }\end{array}$ & $\begin{array}{l}\text { uncordinated walking on } \\
\text { toes, limping }\end{array}$ & $\begin{array}{l}\text { Paralysis, limp, } \\
\text { convulsions, tremor. }\end{array}$ \\
\hline Posture & Normal & Huddled up, stretching & Imbalance, twitching & Fall over, circle \\
\hline $\begin{array}{l}\text { Physical } \\
\text { condition }\end{array}$ & Normal & $\mathrm{BC} 2=$ condition & BC $5=$ obese & $\begin{array}{l}\text { BC1 }=\text { emaciated } \\
\text { BC6 }=\text { extreme obese }\end{array}$ \\
\hline Fur/skin & Normal & $\begin{array}{l}\text { Dry, rough, not shiny } \\
\text { anymore }\end{array}$ & $\begin{array}{l}\text { Piloerection, small } \\
\text { wounds, porfyrie, dry } \\
\text { white skin }\end{array}$ & $\begin{array}{l}\text { Red/black skin, } \\
\text { inflammation, wounds, } \\
\text { loss of fur }\end{array}$ \\
\hline Hydratation & Normal & Loss of skin elasticity & Reduced skin turgor & $\begin{array}{l}\text { Severly reduced turgor }+ \\
\text { sunken eyes }\end{array}$ \\
\hline Breathing & Normal & Fast and superficial & $\begin{array}{l}\text { Fast abdominal breathing } \\
+ \text { audible breathing }\end{array}$ & $\begin{array}{l}\text { Respiratory problems, } \\
\text { cyanosis, breathing with } \\
\text { open mouth }\end{array}$ \\
\hline Faeces/urine & Normal & Moist faeces, polyurie & Diarrhea, abnormal urine & $\begin{array}{l}\text { Uncontrolled diarree, } \\
\text { bloody stool, } \\
\text { obstipation, hematuria }\end{array}$ \\
\hline $\begin{array}{l}\text { Surgical } \\
\text { wound }\end{array}$ & $\begin{array}{l}\text { Normal } \\
\text { healing }\end{array}$ & $\begin{array}{l}\text { Sutures intact, slighty } \\
\text { red/bloody }\end{array}$ & $\begin{array}{l}\text { Dehiscence of wound, } \\
\text { sutures open, fluid } \\
\text { secretion }\end{array}$ & $\begin{array}{l}\text { Severe bleeding, wound } \\
\text { open, severe redness, } \\
\text { necrosis }\end{array}$ \\
\hline Edema & Normal & $\begin{array}{l}\text { Mild abnormal fluid } \\
\text { collections, swollen } \\
\text { appearance }\end{array}$ & $\begin{array}{l}\text { Abnormal large abnormal } \\
\text { fluid collections, ascites }\end{array}$ & $\begin{array}{l}\text { Severe large abnormal } \\
\text { fluid collections }\end{array}$ \\
\hline Necrosis & Normal & Dark skin colouring & $\begin{array}{l}\text { Small dark/black spots, } \\
\text { burning wounds, blisters }\end{array}$ & Big black spots, crusts \\
\hline
\end{tabular}




\section{CHAPTER 7}

\section{Sealing insufficient colonic anastomoses with}

cyanoacrylate tissue adhesive: an in-vivo study

Konstantinos A. Vakalopoulos, Joanna W.A.M. Bosmans, Kevin W.Y. van Barneveld, Ruben R.M. Vogels, Geesien S.A. Boersema, Marion J.J. Gijbels, Johannes Jeekel, Nicole D. Bouvy, Johan F. Lange 


\section{Abstract}

\section{Background}

Tissue adhesives (TA) may be useful to strengthen colorectal anastomoses, thereby preventing anastomotic leakage (AL). Previous studies have identified Cyanoacrylate (CA) TAs as possible colonic anastomotic sealants. This study investigates the protective effects of sealing colonic anastomoses with CA.

\section{Materials and methods}

55 Wistar rats underwent laparotomy and transection of the proximal colon. An anastomosis was created with 4 interrupted sutures followed by either application of Histoacryl Flexible, Omnex, Glubran 2, or no TA seal. An additional control group was included with 12 sutures and no TA seal. After 7 days rats were sacrificed and scored for presence of AL as main outcome. Secondary outcomes were occurrence of bowel obstruction or adhesions, anastomotic bursting pressure and histological evaluation.

\section{Results}

The highest AL rate was found in the Glubran 2 group (7/11), followed by the 4 -sutures group without TA (5/11) and the Omnex group (5/11). Histoacryl Flexible showed the lowest AL rate (2/11). In the control group only 1 rat showed signs of AL. Histologically, the highest influx of inflammatory cells was found in the 4-suture group without TA and for Omnex and Glubran 2. Histoacryl Flexible caused more mature collagen deposition when compared to the other TA groups.

\section{Conclusions}

Histoacryl Flexible showed the lowest leakage rates compared to the other TA groups and to the 4 suture control group. Glubran 2 showed the highest AL rate and a high inflammatory response. Histoacryl Flexible was associated with a higher and more mature collagen deposition, and seems to promote anastomotic healing. 


\section{Introduction}

Anastomotic leakage (AL) in colorectal surgery remains a major problem. Despite years of extensive research, AL is still commonplace, occurring in $5-15 \%$ of colorectal anastomoses ${ }^{1}$.

Over the years, various approaches have been proposed to prevent the onset of AL. Mechanical intraluminal devices creating a magnetic anastomosis ${ }^{2}$, intraluminal sheets of plastic covering the anastomosis ${ }^{3}$ and exotic techniques such as tissue welding 4 have all been proposed, but most were quickly abandoned after their introduction due to inefficacity or high complication rate.

More recently, the idea of sealing an anastomosis externally with a tissue adhesive has been in the spotlight, and has been linked to promising results ${ }^{5}$. The benefit of such a technique is that a surgeon can create an anastomosis in a normal manner, using sutures or staples and performing an intra-operative leak test or other post- anastomotic testing, before applying an extra layer of protection to the serosal surface of the anastomosed colon. Of the various available tissue adhesives (TAs), a special interest has arisen for cyanoacrylate (CA) based tissue adhesives ${ }^{6}$. CA is a type of chemical polymer, also known as 'superglue'. In the early years of CA tissue adhesives, their use was linked to the impairment of anastomotic healing by exothermic polymerization and tissue toxicity7,8. Newer CA formulations have eliminated this highly exothermic curing process by shortening the length of the polymer-chains and adding various additives, also increasing the flexibility of the $\mathrm{CA}^{9}$. Recent studies show that these new CAs are inert when used on the colon, not causing toxic reactions, while maintaining enough elasticity to cope with peristaltic movement and intraluminal forces ${ }^{10}$.

Several experimental studies have been performed using CAs to prevent AL, yielding ambiguous results ${ }^{5,6}$. This may be due to a large spectrum of experimental methodology in the various studies, in which large differences exist in the used animal models, TA dosage and experimental end-points, which is a well-recognized problem in the field of experimental research on colorectal anastomoses ${ }^{11}$.

Previous research regarding TAs and colonic healing has proposed a stepwise approach in which a large number of TAs have been evaluated following a similar experimental protocol, yielding promising results for CA based TAs ${ }^{12}$.

In the current study we use a rat model to simulate high rates of $\mathrm{AL}$, based on the creation of a mechanically insufficient colon anastomosis, which we seal with a protective barrier of one of 3 CA formulations. The aim of this study is to identify 
promising CAs for the prevention of $\mathrm{AL}$, by blocking the intra-peritoneal leak of bowel contents.

\section{Methods}

\section{Study design}

Three clinically available CAs were included in this study. These TAs were chosen based on their mechanical and rheological profiles as derived from previous research ${ }^{10}$. TA composition and manufacturer details are listed in table 1 . A positive control group, with a 12-suture colonic anastomosis was used, as well as a negative 4-suture control group, without a TA seal. The included study groups are described in Table 7.1. Rat allocation was performed in a randomized manner using a lottery system. Data are reported according to the ARRIVE guidelines (see also supplemental data) ${ }^{13}$. A power analysis was calculated based on a reduction of $20 \%$ ( $\delta$ ) in inflammation, as scored on histological data, between the different experimental groups with a variance of $\pm 16 \%(\sigma)$. The number of animals per group was calculated as follows: $(\alpha: 0.05, \pi: 0.8,2$-sided testing): $\mathrm{n}=2(\mathrm{z} \alpha / 2$ $\mathrm{z \pi}) 2^{*}(\sigma / \delta) 2, \mathrm{n}=2(1.96+0.84)^{2 *}(16 / 20)^{2}, \mathrm{n}=15.7 * 0.64=10.03 \approx 11$.

Table 7.1 Study groups.

\begin{tabular}{lllll}
\hline Group & Name & $\begin{array}{l}\text { Anastomotic } \\
\text { technique }\end{array}$ & Tissue adhesive (TA) & Manufacturer \\
\hline 1 & $\begin{array}{l}\text { Control } \\
\text { No TA } \\
\text { (negative control) }\end{array}$ & $\begin{array}{l}\text { 12 sutures } \\
\text { 4 sutures }\end{array}$ & - & N/A \\
& Histoacryl Flex & 4 sutures & n-butyl-2-cyanoacrylate & $\begin{array}{l}\text { B. Braun (Tuttingen, } \\
\text { GER) }\end{array}$ \\
4 & Omnex & 4 sutures & $\begin{array}{l}\text { 2-octyl-cyanoacrylate/ butyl } \\
\text { lactoyl cyanoacrylate } \\
\text { n-butyl-2-cyanoacrylate and }\end{array}$ & $\begin{array}{l}\text { Sommerville, NJ, USA) } \\
\text { GEM S.r.l. (Viarregio, } \\
\text { IT) }\end{array}$ \\
\hline
\end{tabular}

\section{Animals}

Male specific-pathogen-free Wistar rats (250-350 g) were housed at the Central Animal Facility of the Maastricht University Medical Centre, the Netherlands. Rats were housed according to standard laboratory conditions, including individually ventilated cages with unrestricted access to standard rat chow and water. The experimental protocol complied with the Dutch Animal Experimentation Act and was approved by the local Animal Experimental Committee. 


\section{Surgical procedure}

Experienced researchers, certified for animal research, performed all surgical procedures. Rats received buprenorphine $0.1 \mathrm{mg} / \mathrm{kg}$ (Temgesic, Schering-Plough, USA) pre-operatively for analgesia which was repeated when necessary postoperatively. Anesthesia was induced by inhalation of isoflurane $5.0 \mathrm{vol} \%$ (Forene, Abbott Laboratories, USA), followed by a maintenance dose of 2.5 vol\%. The abdominal skin was shaved, disinfected with iodine $1 \%$ and covered with sterile drapes. A 5-cm midline incision was made through which the cecum was identified and exteriorized onto sterile gauzes. The ascending colon was transected $2-\mathrm{cm}$ distally to the cecum, without damaging the mesenteric vessels. An insufficient end-to-end colonic anastomosis was created using 4 evenly distributed polypropylene 6/0 sutures (Prolene, Ethicon, Johnson \& Johnson, USA). After construction of the anastomosis, in the TA groups, $0.025 \mathrm{ml}$ of TA was applied evenly to the anastomotic site using the provided applicators. In the control group, 12 sutures were used instead of 4 , obtaining a sufficient anastomosis. Postoperative hydration was provided by injecting a bolus of $5 \mathrm{~mL}$ sterile saline solution $\left(37^{\circ} \mathrm{C}\right)$ into the abdominal cavity after repositioning of the abdominal contents. The abdominal wall was closed using a running suture of polyglactine 4/0 (Vicryl, Ethicon, Johnson \& Johnson, USA). Skin closure was performed with an intracutaneous running suture of polyglecaprone 4/0 (Monocryl, Ethicon, Johnson \& Johnson, USA). Postoperatively, a daily evaluation of all animals was carried out. Animal weight and signs of distress were noted. In case of severe distress/illness the animals were sacrificed prior to the completion of the follow-up time.

\section{Outcome measures}

The main outcome of the study was anastomotic leakage (AL), including macroscopic anastomotic dehiscence, fecal peritonitis or large anastomotic abscesses. After 7 days or when humane endpoints were reached, rats were sacrificed using an overdose of carbon dioxide. The abdomen was re-opened and the abdomen was macroscopically inspected for signs of leakage or TA-related complications, that is, the presence of intraperitoneal abscess or fecal matter and ileus formation. Abscess formation was scored using the following parameters: 1) one or several millimetric abscesses; 2) abscess up to $1 / 4$ of anastomotic circumference; 3) Large abscess $>1 / 4$ of anastomotic circumference; 4) intraabdominal abscess formation. Based on our previous research, in which we found that an abscess score of 1 was not associated with any clinical complications and therefore not clinically significant, we defined AL in this study as the presence of fecal peritonitis or an abscess score of $>2^{12,20}$. The Zühlke score, which depicts the tenacity of intra-abdominal adhesions was also determined ${ }^{14}$. 


\section{Anastomotic bursting pressure}

To measure anastomotic bursting pressure $(\mathrm{ABP})$, a plastic tube was inserted into the colon proximally to the anastomotic site, and ligated with a single polyglactine $4 / 0$ suture. The distal colonic segment was clamped to ensure an airtight compartment. Pressure was gradually increased in the anastomotic compartment using an automatic pressure pump (IDEE, Maastricht, the Netherlands). ABP was monitored and recorded using a digital manometer until the colon bursted/air bubbles appeared. The maximum bursting pressure was recorded for each rat.

\section{Histological evaluation}

After ABP testing, the anastomotic segment was subsequently resected and prepared for histological evaluation. Tissue samples were embedded in paraffin and cut in $4 \mu \mathrm{m}$ sections. To evaluate morphology of cells, standard hematoxylineosin (H\&E) staining was performed. Specimens were scored based on inflammation, fibroblast activity, collagen deposition and neoangiogenesis according to the Ehrlich and Hunt numerical scale (0-4) as modified by Phillips et al: 0 = no evidence, 1 = occasional evidence, 2 = light scattering, 3 = abundant evidence, 4 = confluent cells or fibers ${ }^{15}$. All slides were evaluated by an experienced pathologist (MG) who was blinded for the experimental groups.

\section{Evaluation of collagen formation}

Tissue sections were stained for collagen using Picro Sirius red as previously described ${ }^{16}$. The results between experimental groups were compared, no collagen staining was performed in de 12-suture control groups, as these findings are wellknown and have been reported in numerous recent studies ${ }^{17-19}$. In short, sections were exposed to a $0.1 \%$ solution of Sirius red in saturated aqueous picric acid for 90 minutes, followed by 2 min of washing in $0.01 \mathrm{~N} \mathrm{HCl}$, dehydration and mounted with Entellan. Images of the anastomotic region were taken (200x magnification) using cross polarization light microscopy (Leica DM5000B, Leica Microsystems, Switzerland). Collagen percentages of anastomotic tissue were calculated. Maturity level of collagen was estimated by calculating the red (mature fibers, collagen type I) versus green (immature fibers, collagen type III) area ratio using the Qwin morphometry-system (Leica QWin V3.5.1, Leica Microsystems).

\section{Statistics}

One-way ANOVA was used in case of continuous variables, with a Bonferroni posthoc test. A $\chi^{2}$-test or Fisher's exact was used in case of categorical variables. A $P$-value $\leq 0.05$ was considered statistically significant. All analyses were performed 
using IBM SPSS Statistics, version 21.0 for Mac (IBM SPSS, USA), while graphs were composed using GraphPad Prism, version 5.0a for Mac (GraphPad Software, USA).

\section{Results}

\section{Anastomotic Leakage}

Both in the 4-suture non-TA and Glubran 2 groups, one rat died prior to completion of the follow-up period due to fecal peritonitis caused by AL. Except for these two rats, AL only consisted of the presence of anastomotic abscesses. In the 12-suture control group AL occurred in one rat, associated with an abscess score of 1 . In the 4-suture non-TA group 4 rats showed signs of AL, with an abscess score of 2 in 3 rats and an abscess score of 4 in 1 rat. In the TA groups there was a large difference in AL-rate. Glubran 2 had the highest AL-rate, consisting of 1 total anastomotic dehiscence and subsequent fecal peritonistis, and 6 cases of abscess formation. Abscess scores in this TA group ranged from 1 to 4 . Five rats in the Omnex group and two rats in the Histoacryl Flexible group showed signs of AL, with maximum abscess scores of 4 and 2, respectively. Statistical analysis shows that Glubran 2 had a significantly higher amount of abscesses when compared to the positive control group $(P=0.013)$ and Histoacryl Flexible $(P=0.049)$. A synopsis of AL-rates is provided in Figure 7.1A.

\section{Clinical outcomes}

Discomfort in the various groups is reflected by changes in weight loss throughout the follow-up period (Figure 7.1B). The negative control group, in which no TA was used, showed the highest rate of weight loss. For the TA groups, Glubran 2 group showed the most weight loss at 7 days, significantly higher than the control group $(P<0.01)$ and the Histoacryl Flexible group $(P<0.01)$.

Mechanical ileus rate varied significantly between the TA groups, with Glubran 2 showing the highest ileus rate, significantly higher than when compared to the negative control group $(P=0.01)$. The number and Zuhlke score of adhesions did not differ significantly between experimental groups. A synopsis of the clinical outcomes in presented in Table 7.2. 
A

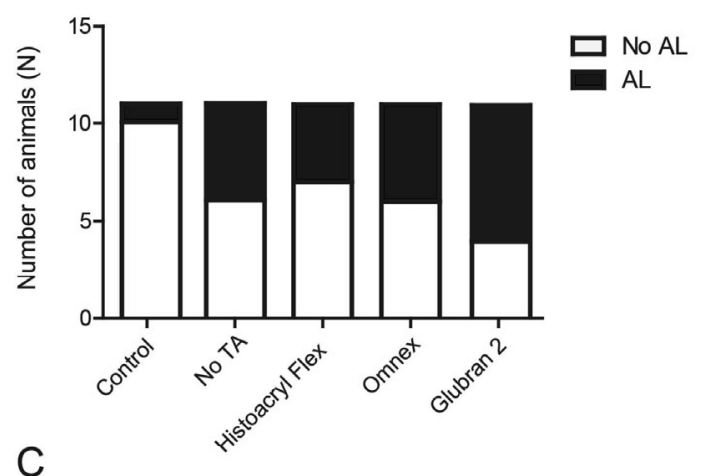

B

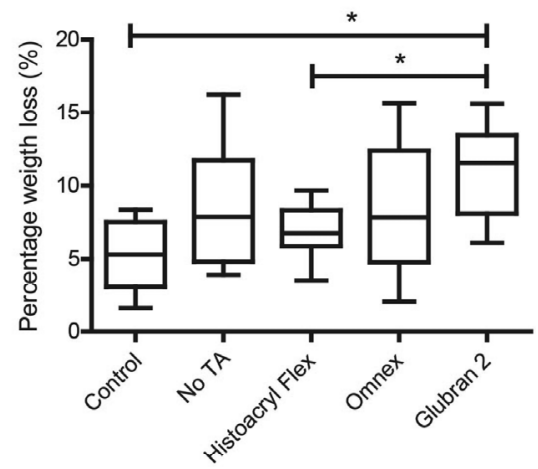

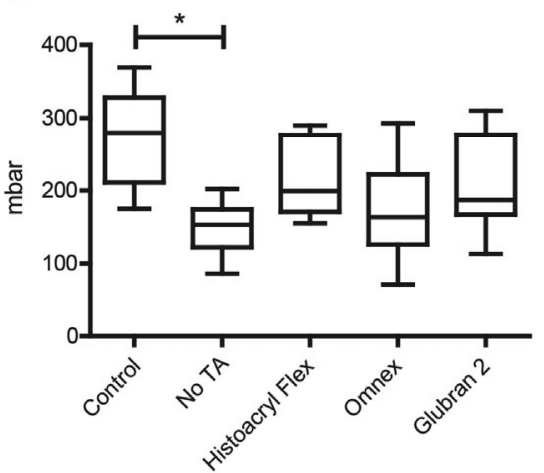

Figure 7.1 A) Histoacryl Flexible had the lowest amount of anastomotic leakage (2/11) compared to the other intervention groups Omnex 5/11 and Glubran2 7/11 and the no TA group 5/11. The control group with 12 sutures only showed one case of $\mathrm{AL}(\mathrm{X} 2=9.43, P=0.05)$. B) Weight loss was monitored as a measure of discomfort and the percentage weight loss was highest in the no TA group, followed by the Glubran 2 group with was significantly higher than the Histoacryl Flexible group and the control group. C) No significant differences were found between the experimental groups regarding anastomotic bursting pressure. Control group (12 sutures) and the group without TA showed significanly different $\operatorname{ABP}(P=0.004)$.

\section{Anastomotic Bursting Pressure (ABP)}

The highest $\mathrm{ABP}$, as depicted in Fig. 1C, was found in the 12-suture control group $(272 \mathrm{mmHg} \pm 70$ ) and differed significantly from the 4-suture no TA control group $(147 \mathrm{mmHg} \pm 37, P<0.01$ ). The use of TA resulted in an increase in ABP in all three TA groups, however no statistically significant differences were found. The highest increase in ABP was found in the Histoacryl Flexible group (217 mmHg \pm 53 ), followed by the Glubran 2 group $(205 \mathrm{mmHg} \pm 67)$. Omnex showed the lowest ABP of the TA groups $(173 \mathrm{mmHg} \pm 69)$. 
Table 7.2 Synopsis of clinical outcomes.

\begin{tabular}{lccccc}
\hline Group & $\begin{array}{c}\text { Number } \\
\text { of rats }\end{array}$ & $\begin{array}{c}\text { Fecal } \\
\text { peritonitis* }\end{array}$ & $\begin{array}{c}\text { Mechanical } \\
\text { Ileus* }\end{array}$ & $\begin{array}{c}\text { Adhesions, } \\
\text { total (mean) }\end{array}$ & $\begin{array}{c}\text { Median } \\
\text { Zuhlke score }\end{array}$ \\
\hline 1) 12 sutures & 11 & 0 & 1 & 4,4 & 3 \\
2) 4 sutures & 11 & 1 & 0 & 5 & 3 \\
3) Histoacryl Flex & 11 & 0 & 1 & 5,5 & 3 \\
4) Omnex & 11 & 0 & 1 & 5,7 & 3 \\
5) Glubran 2 & 11 & 1 & 5 & 5,8 & 3 \\
\hline
\end{tabular}

*Number of affected rats.

\section{Histological evaluation}

The use of a 4-suture anastomosis, with or without the presence of a TA, led to more inflammation when compared to the 12-suture control group (Figure 7.2A). A significantly higher inflammation score was found in the Omnex and Glubran 2 groups when compared to the control group $(P<0.01)$. Significantly more collagen deposition was found in the Histoacryl Flexible group and the Omnex group in comparison with the control group (Figure 7.2B, $P<0.01$ ). Fibroblast activity and neoangiogenesis did not differ between the experimental groups (Figure 7.2C \& 7.2D).

Picro Sirius red staining, which depicts the level of collagen maturity, of the anastomotic region showed comparable percentages of collagen for all groups (Figure 7.3A, $P=0.214$ ). When focusing on collagen maturity, a significant difference was found in red/green ratios between the Histoacryl Flexible and Glubran 2 group (Figure 7.3B, $P<0.05$ ), illustrating a higher level of healing in the Histoacryl Flexible group. 
A

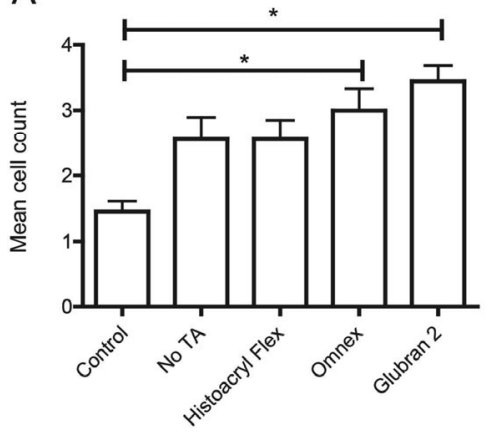

B

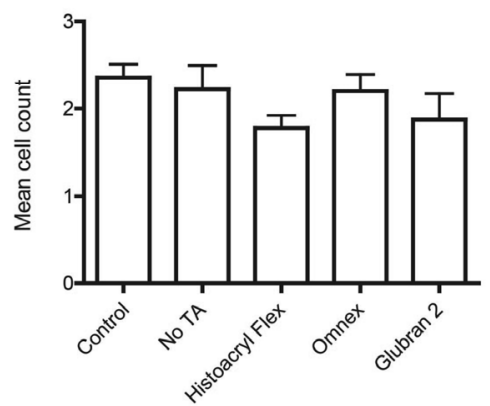

C

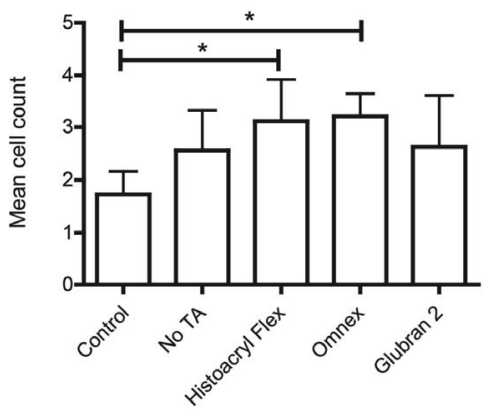

$\mathrm{D}$

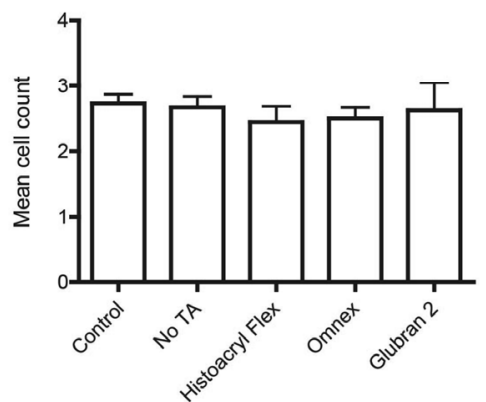

Figure 7.2 A) significantly more inflammation occurred in the Omnex and Glubran 2 group compared to the control group. B) No differences were found between groups regarding fibroblast acitivity. C) More collagen deposition was found in the Histoacryl group and the Omnex group compared to the control group. Neoangiogenesis (D) did not differ between the experimental groups.

A

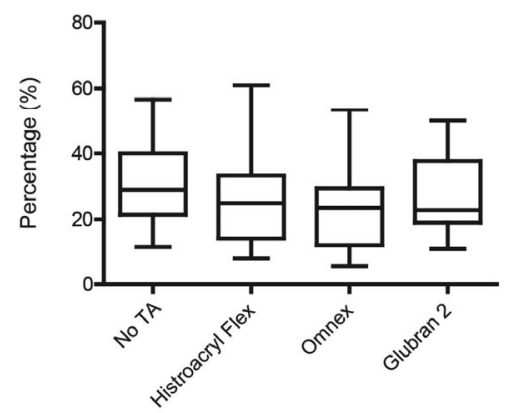

B

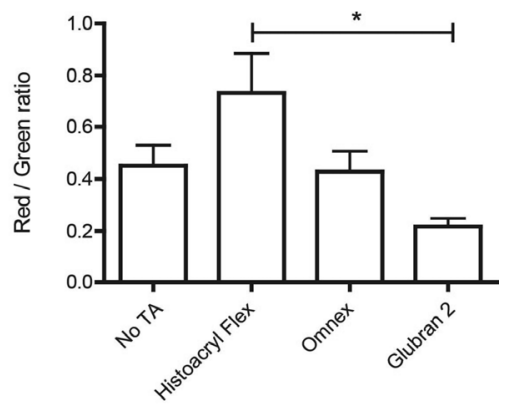

Figure 7.3 A) No differences were found between groups in the relative collagen area (quantified as the percentage of total tissue surface). B) Maturity of collagen was estimated by calculating the red/green ratio, which was significantly higher in the Histoacryl Flexible group compared to the Glubran 2 group, indicating more mature collagen. 


\section{Discussion}

The sealing of colonic anastomoses with tissue adhesives (TAs) has been proposed as a promising method for the prevention of anastomotic leakage by forming a mechanical barrier that can protect from the leakage of intraluminal contents into the peritoneum, giving the anastomosis time to heal20. Of the large amount of available TAs, recent research has provided evidence that cyanoacrylate (CA) TAs may be useful in the prevention of $\mathrm{AL}^{6}$. In the current study, we selected three clinically available CAs to seal insufficient colonic anastomoses in a rat model, also including a positive control group in which a 12 suture colonic anastomosis was used as well as a negative, 4-suture, control group. We evaluated the effectiveness of each TA in the prevention of $\mathrm{AL}$, also taking into account mechanical strength and histological profile.

Overall, there were large differences between AL rates of the various groups.. In this study AL was mostly presented as peri-anastomotic abscess formation. The described abscess score was used to score the severity and amount of abscesses; generally an abscess score of $<2$ was not associated with any clinical symptoms, and therefore not considered clinically relevant. Histoacryl Flexible, a combination of n-butyl-2-cyanoacrylate and a softener, showed the lowest rate of AL, occurring in 2 rats. Furthermore, the maximum abscess score with Histoacryl Flexible was lower than with the other TAs, and consisted only of punctiform abscesses around the anastomosis, which did not have any clinical consequences. The histological evaluation showed that this TA resulted in the least inflammation and the highest level of collagen formation and healing of the TA groups. Overall, Histoacryl Flexible showed promising results, with an AL rate comparable to the 12-suture control group, with positive clinical outcomes and improved histological assessment. This TA seems to be a safe and effective colonic sealant.

Glubran 2, based on an n-butyl-2-cyanoacrylate and methacryloxy sulfolane mixture, showed the poorest results in our study. In terms of AL, the use of this TA resulted in one case of premature death due to fecal peritonitis as well as the highest rates of abscess formation and severity. Furthermore, its use was associated with higher incidence of mechanical ileus, occurring in five rats, significantly higher than in the 4-suture control group. Rats in this group showed the most weight loss of all study groups. Histological analysis associated Glubran 2 use with the highest degree of inflammation, and a significantly more premature collagen ratio, indicating less healing capability. Glubran 2 induced an extended inflammatory response with mild local muscle lysis as deep as the submucusal colonic layer. This finding was also reported in a previous study by Kayaoglu 21 . Omnex, a 2-octyl-cyanoacrylate / butyl-lactoyl-cyanoacrylate mixture, showed 
similar results to the negative control group in terms of AL rate, clinical effects, mechanical strength and histological analysis. Presence of this TA thus did not improve outcomes nor lead to any complications when used on the colon.

Results of this study are in line with previous research on the use of CA in experimental AL models. In a previous study from our group the same set of CAs were applied on rat colon without the presence of a colonic defect and followed for 1 or 4 weeks $^{12}$. That study showed that Histoacryl Flexible retained the lowest complication rate and a relatively inert histological profile, with a limited local host reaction and an increase in inflammatory markers at 7 days, a finding which did not persist at 28 days when no ongoing inflammation reaction was found. In the present study, Histoacryl Flexible was associated with a higher inflammatory reaction than the 12-suture controle group at 7 days, comparable to the 4-suture no-TA group. The longterm inflammatory reaction of this TA in an anastomosis model may be an interesting subject for further research.

All three TAs included are modern CAs, clinically in use. When examing the chemical composition of each CA (as depicted in table 1), one may note that the main ingredients of the included CAs are similar to one another. Glubran 2 (n-butyl2-cyanoacrylate/methacryloxysulfolane) does not differ considerably from either Histoacryl Flexible (n-butyl-2-cyanoacrylate) or Omnex (n-octylcyanoacrylate/butyl/lactoyl acrylate), while differences exist in the various additives and softeners. As reported in previous research, Glubran 2 elicits a significantly higher inflammatory response than the other included Cas, and this may possibly be attributed to methacryloxysulfolane, an additive in Glubran 2 that increases flexibility 6 . This finding should be addressed in future studies.

Our study has several limitations. Firstly, we opted for a follow-up time of 7 days to evaluate short-term effectiveness after creation of an anastomosis. Therefore, we cannot comment on the long-term safety of CA use on colonic anastomosis. This may be in part extrapolated from our previous studies, however, a future objective would be to use several time-points. By doing this, one could also evaluate the clinical relevance of the (sub)clinical local abscesses we encountered, which, in fact, may reflect perioperative spillage of bowel contents instead of postoperative anastomotic leakage. Secondly, as a large number of colorectal anastomoses are stapled nowadays, a study on the interaction between CA and a stapled anastomosis may an interesting step for further research.

In conclusion, this study shows that the use of Glubran 2 was directly associated with poor outcomes and does not seem to be a suitable TA for the sealing of colonic anastomosis. Furthermore, we found limited evidence of a protective effect of the 
use of Histoacryl Flexible as an anastomotic sealant. The use of this CA showed a trend for the decrease of $\mathrm{AL}$, an increase in mechanical strength and stimulation of wound healing when compared to the other CAs and to a negative control group. It was associated with an incidence of AL comparable to a standard, 12 suture anastomosis and its potential should be further evaluated in future research. 


\section{References}

1. Kingham TP, Pachter HL. Colonic anastomotic leak: Risk factors, diagnosis, and treatment. J Am Coll Surg. 2009;208(2):269-278.

2. Xu ZF, Li ZJ, Sun YL, et al. A novel spherical magnetic compression device for colorectal anastomosis in a swine model. J Laparoendosc Adv Surg Tech A. 2015;25(4):323-329.

3. Bakker IS, Morks AN, Hoedemaker HO, et al. The C-seal trial: Colorectal anastomosis protected by a biodegradable drain fixed to the anastomosis by a circular stapler, a multi-center randomized controlled trial. BMC Surg. 2012;12:23-2482-12-23.

4. Ho YH, Ashour MA. Techniques for colorectal anastomosis. World J Gastroenterol. 2010;16(13):1610-1621.

5. Vakalopoulos KA, Daams F, Wu Z, et al. Tissue adhesives in gastrointestinal anastomosis: A systematic review. J Surg Res. 2013;180(2):290-300.

6. Wu Z, Boersema GS, Vakalopoulos KA, et al. Critical analysis of cyanoacrylate in intestinal and colorectal anastomosis. J Biomed Mater Res B Appl Biomater. 2014;102(3):635-642.

7. Gennaro AR, Moreira CA. Nonsuture colonic anastomosis. Dis Colon Rectum. 1976;19(3):245-249.

8. Quinn J. Tissue Adhesives in Clinical Medicine. 2005. PMPH-USA.

9. Scognamiglio F, Travan A, Rustighi I, et al. Adhesive and sealant interfaces for general surgery applications. J Biomed Mater Res B Appl Biomater. 2016;104(3):626-39

10. Vakalopoulos KA, Wu Z, Kroese L, et al. Mechanical strength and rheological properties of tissue adhesives with regard to colorectal anastomosis: An ex vivo study. Ann Surg. 2015;261(2):323-331.

11. Bosmans JW, Moossdorff M, Al-Taher M, van Beek L, Derikx JP, Bouvy ND. International consensus statement regarding the use of animal models for research on anastomoses in the lower gastrointestinal tract. Int J Colorectal Dis. 2016 Mar 10.

12. Vakalopoulos $\mathrm{KA}, \mathrm{Wu}, \mathrm{Z}$. Clinical effects, mechanical strength and immunohistopathological analysis of surgical tissue adhesives after use on the colon: An in vivo study. J Biomed Mater Res Part B 2016: Jan 24.

13. Kilkenny C, Browne W, Cuthill IC, Emerson M, Altman DG, NC3Rs Reporting Guidelines Working Group. Animal research: Reporting in vivo experiments: The ARRIVE guidelines. J Gene Med. 2010;12(7):561-563.

14. Zuhlke HV, Lorenz EM, Straub EM, Savvas V. Pathophysiology and classification of adhesions. Langenbecks Arch Chir Suppl II Verh Dtsch Ges Chir. 1990:1009-1016.

15. Kanellos D, Blouhos K, Pramateftakis MG, et al. Effect of 5-fluorouracil plus interferon on the integrity of colonic anastomoses covering with fibrin glue. World J Surg. 2007;31(1):186-191.

16. Bloemen JG, Schreinemacher MH, de Bruine AP, Buurman WA, Bouvy ND, Dejong CH. Butyrate enemas improve intestinal anastomotic strength in a rat model. Dis Colon Rectum. 2010;53(7):1069-1075.

17. Hoeppner J, Wassmuth B, Marjanovic G, Timme S, Hopt UT, Keck T. Anastomotic sealing by extracellular matrices (ECM) improves healing of colonic anastomoses in the critical early phase. J Gastrointest Surg. 2010;14(6):977-986.

18. van der Vijver RJ, van Laarhoven CJ, de Man BM, Lomme RM, Hendriks T. The effect of fibrin glue on the early healing phase of intestinal anastomoses in the rat. Int J Colorectal Dis. 2012;27(8):11011107.

19. Grommes J, Binnebosel M, Klink CD, et al. Comparison of intestinal microcirculation and wound healing in a rat model. J Invest Surg. 2013;26(1):46-52.

20. Vakalopoulos KA. Sutureless closure of colonic defects with tissue adhesives: An in-vivo study in the rat. American Journal of Surgery. 2016 (accepted for publication).

21. Kayaoglu HA, Ersoy OF, Ozkan N, Celik A, Filiz NO. Effect of n-butyl-2-cyanoacrylate on high-risk colonic anastomoses. Kaohsiung J Med Sci. 2009;25(4):177-183. 


\section{S 7.1 Supplemental information according to ARRIVE guidelines}

\section{Ethical statement}

- The experimental protocol complied with the Dutch Animal Experimental Act and was approved by the Animal Experimental Committee of Maastricht University Medical Center. Protocols for institutional animal use and care guidelines were followed (permit DEC 2012-055)

\section{Study design}

- In total there were 55 rats used in this study. We used 11 rats per experimental group, and 5 groups.

\section{Experimental procedures}

- $\quad$ For surgical procedure, see manuscript. This was carried out in the rat operating room of the animal facility of Maastricht University under semisterile conditions.

\section{Experimental animals}

- Male Wistar rats (RccHan:WIST) were purchased from Harlan laboratories, the Netherlands. Rats had an average weight of $307 \mathrm{~g}$ on the day of surgery (SD 16.7).

- We have chosen for male rats, since it is known that female estrogens and androgens have an important influence on wound-healing (Ashcroft GS, et al. Estrogen modulates cutaneous wound healing by downregulating macrophage migration inhibitory factor. The Journal of clinical investigation. 2003, 111:1309-1318.)

\section{Housing and husbandry}

- An acclimatization period of one week was observed prior to the start of the experiment.

- $\quad$ Rats were kept under standard conditions and were provided with food and water ad libitum. Rats were housed 2 animals per cage. The general health of rats was monitored several times per week for signs of inflammation and animals were weighed once per week. During the experiment animals were weighed daily and scored for discomfort twice daily (every morning and every evening). In case of discomfort, additional pain treatment was administered by giving buprenorphine $0.05 \mathrm{mg} / \mathrm{kg}$ s.c.

- Discomfort was scored using a standard scheme (zie below). Humane endpoints were defined according to Roughan \& Flecknell (Roughan, J. V. \& 
Flecknell, P. A. Behavioural effects of laparotomy and analgesic effects of ketoprofen and carprofen in rats. Pain 90, 65-74 (2001).

- Humane endpoints are defined as:

○ Significant weight loss $>20 \%$

- Fever (temperature is only measured on indication)

- Tachypnoe

- Significant differences in behavior:

- Lethargia

- Twitching: random spasms of the muscles, can be seen when animals are asleep or inactive in huddled up position.

- Walking: unable to stand on four legs, wobbly walk

- Huddled up posture: showing a concave abdominal side. Can be seen when walking/sitting.

- Signs of severe dehydration

- Severe diarrhea

- Severely inflammed surgical wound

$\circ$ Cachexia

Allocating animals to experimental groups

- Animals were randomized by means of throwing a dice. Groups were divided into 1-5 (see table 1). For each animal the dice was thrown and the rat received the assigned treatment. Whenever 6 was thrown, the dice was rolled again.

\section{Numbers analysed}

- All analyses were performed according to an intention-to-treat analysis.

However, since there were adverse events and deaths prior to follow-up, for $\mathrm{ABP}$ and histological assessment these animals were not taken into account. 
Welfare scoring rat model

\begin{tabular}{|c|c|c|c|c|}
\hline \multicolumn{5}{|l|}{ Description } \\
\hline & 0 & 1 & 2 & 3 \\
\hline Activity & Normal & Isolated, less active & Inactive & $\begin{array}{l}\text { Somnulent, stupor, } \\
\text { coma, lifeless }\end{array}$ \\
\hline Behavior & Normal & $\begin{array}{l}\text { Back arching, } \\
\text { twitching, shivering } \\
\text { Once/10 min }\end{array}$ & $\begin{array}{l}\text { Back arching, twitching, } \\
\text { shivering }\end{array}$ & $\begin{array}{l}\text { Stereotype } \\
\text { behavior, auto } \\
\text { mutilation, } \\
\text { aggressive behavior }\end{array}$ \\
\hline Gait & Normal & $\begin{array}{l}\text { Mildly } \\
\text { uncoordinated/ } \\
\text { Abnormality }\end{array}$ & $\begin{array}{l}\text { uncordinated walking on } \\
\text { toes, limping }\end{array}$ & $\begin{array}{l}\text { Paralysis, limp, } \\
\text { convulsions, tremor. }\end{array}$ \\
\hline Posture & Normal & $\begin{array}{l}\text { Huddled up, } \\
\text { stretching }\end{array}$ & Imbalance, twitching & Fall over, circle \\
\hline $\begin{array}{l}\text { Physical } \\
\text { condition }\end{array}$ & Normal & $\mathrm{BC} 2=$ condition & BC5 = obese & $\begin{array}{l}\text { BC1 }=\text { emaciated } \\
\text { BC6 }=\text { extreme obese }\end{array}$ \\
\hline Fur/skin & Normal & $\begin{array}{l}\text { Dry, rough, not shiny } \\
\text { anymore }\end{array}$ & $\begin{array}{l}\text { Piloerection, small wounds, } \\
\text { porfyrie, dry white skin }\end{array}$ & $\begin{array}{l}\text { Red/black skin, } \\
\text { inflammation, } \\
\text { wounds, loss of fur }\end{array}$ \\
\hline Hydratation & Normal & Loss of skin elasticity & Reduced skin turgor & $\begin{array}{l}\text { Severly reduced } \\
\text { turgor + sunken } \\
\text { eyes }\end{array}$ \\
\hline Breathing & Normal & Fast and superficial & $\begin{array}{l}\text { Fast abdominal breathing + } \\
\text { audible breathing }\end{array}$ & $\begin{array}{l}\text { Respiratory } \\
\text { problems, cyanosis, } \\
\text { breathing with open } \\
\text { mouth }\end{array}$ \\
\hline Faeces/urine & Normal & Moist faeces, polyurie & Diarrhea, abnormal urine & $\begin{array}{l}\text { Uncontrolled } \\
\text { diarree, bloody } \\
\text { stool, obstipation, } \\
\text { hematuria }\end{array}$ \\
\hline Surgical wound & $\begin{array}{l}\text { Normal } \\
\text { healing }\end{array}$ & $\begin{array}{l}\text { Sutures intact, slighty } \\
\text { red/bloody }\end{array}$ & $\begin{array}{l}\text { Dehiscence of wound, } \\
\text { sutures open, fluid secretion }\end{array}$ & $\begin{array}{l}\text { Severe bleeding, } \\
\text { wound open, severe } \\
\text { redness, necrosis }\end{array}$ \\
\hline Edema & Normal & $\begin{array}{l}\text { Mild abnormal fluid } \\
\text { collections, swollen } \\
\text { appearance }\end{array}$ & $\begin{array}{l}\text { Abnormal large abnormal } \\
\text { fluid collections, ascites }\end{array}$ & $\begin{array}{l}\text { Severe large } \\
\text { abnormal fluid } \\
\text { collections }\end{array}$ \\
\hline Necrosis & Normal & Dark skin colouring & $\begin{array}{l}\text { Small dark/black spots, } \\
\text { burning wounds, blisters }\end{array}$ & $\begin{array}{l}\text { Big black spots, } \\
\text { crusts }\end{array}$ \\
\hline
\end{tabular}




\section{Part IV}

Potential disruption of the balance

between anastomotic healing and adhesion formation 


\section{CHAPTER 8}

\section{Effects of glycerol as a plasticizer in}

biomaterials designed for intra-abdominal use

J.W.A.M. Bosmans, A.C.H.M. Jongen, R.D.W. Vaes,

F. Scognamiglio, M. Borgogna, A. Travan, E. Marsich, M.J.J. Gijbels, N.T.H. Hoebers, J.W. Jocken, I. Donati,, S. Paoletti, N.D. Bouvy 


\section{Abstract}

\section{Introduction}

Plasticizers are commonly used to improve the mechanical characteristics of biomaterials. We studied two biomaterials designed as membranes able to reduce colonic anastomotic leakage (AL), which were plasticized with glycerol, a frequently used plasticizer. The pathophysiology of the observed extensive patch-host interaction was investigated.

\section{Methods}

Animal experiments were performed in male Wistar rats, which received a colonic anastomosis using 4 sutures (leakage model, $n=30$ ) or 12 sutures (healing model, $n=36$ ). In both cases, two membranes, either containing Hyaluronic Acid-Alginate-Glycerol or GelatinGlycerol were used to cover the anastomosis. A chorioallantoic membrane (CAM) assay was performed assessing the pro-inflammatory effects of the materials employed. In vitro studies were performed evaluating viability of human multipotent adipose derived stem cells in the presence of increasing concentrations of glycerol.

\section{Results}

Both patches containing glycerol induced large abscess formation and severe adhesions in the leakage model $(n=20)$, while these parameters were present only at a limited extent in control animals $(n=10)$. In animals that did not show AL, encapsulation of the glycerol patches was evident. CAM assay identified glycerol as the cause of adverse patch-host reactions. Cell culture revealed a decrease of adipocyte-viability upon increase of glycerol concentration beyond $2 \%$.

\section{Conclusions}

Glycerol-plasticized patches induced adverse events in a rat model. CAM assay data demonstrated that glycerol compromises the biocompatibility of the patch while in vitro data showed that adipocytes are less viable in the presence of glycerol $(>2 \%)$. Caution should be taken when selecting glycerol as plasticizer in biomaterials designed for intra-abdominal use. 


\section{Introduction}

Anastomotic leakage (AL) is a complication occurring in up to $10 \%$ of patients undergoing colorectal surgery, a type of surgery that is commonly used to remove the affected part of intestine in, e.g. colorectal cancer or inflammatory bowel disease. AL causes higher rates of morbidity and mortality in patients ${ }^{1,2}$, making AL a dreaded complication for the surgeon. It is commonly accepted that AL is a consequence of improper anastomotic healing ${ }^{3}$. A conceivable solution to prevent or limit the occurrence of AL is the use of an external patch composed of bioactive biopolymers, which should stimulate a fast cicatrization of the anastomosis, thus preventing intestinal content along with bacteria from spilling into the abdominal cavity.

In the EU AnastomoSEAL project ${ }^{4}$, two promising membranes consisting of natural-based biopolymers were proposed for reinforcement of the anastomotic line, one based on hyaluronan and alginate $^{5}$ and one based on gelatin due to its hemostatic characteristics ${ }^{6}$.

The choice of Hyaluronan (HA) as a bioactive component to be delivered to the anastomosis originated from its ability to promote neovascularization, to enhance the process of scarring and from its beneficial effects on cell proliferation in several tissues 7,8 . Alginate is widely used in biomedical applications due to its excellent biocompatibility and low toxicity ${ }^{9,10}$. The combination of HA and alginate is of particular interest as it combines the gel-forming ability of the biologically inert alginate with the biological activity of $\mathrm{HA}^{11,12}$. A recent study on a hyaluronanalginate (HA-A) membrane demonstrated that the HA released by such a device is able to simulate both proliferation and migration of fibroblasts in vitro, leading to the promising hypothesis that it could accelerate anastomotic healing5. Moreover, a similar hyaluronan-based membrane was recently shown in vivo not to exert any adverse reaction in direct contact with intestinal serosa ${ }^{13}$.

Porcine-derived gelatin is widely used by surgeons as hemostatic material to stop bleeding in wound surfaces ${ }^{6}$. It can be postulated that gelatin enhances anastomotic healing, mainly by achieving quick hemostasis assisting the wound healing process ${ }^{14}$. This aim, early hemostasis, is also the rationale behind the use of fibrin sealants, which are designed to mimic the final steps of the blood coagulation cascade, forming a stable physiological fibrin clot leading to early hemostasis ${ }^{15}$. While the theory behind it seems promising, fibrin sealants have not shown a convincing positive effect on the healing of gastrointestinal anastomoses ${ }^{16}$, so the need for a successful alternative still exists. 
The addition of a plasticizer agent to biodegradable blend films represents a feasible approach to enhance the pliability of biopolymer-based membranes ${ }^{17}$. Glycerol is commonly recognized as one of the most suitable plasticizers, since it has low molecular weight with low volatility, causing an increase in free volume of the polymer matrices as well as enhancement of molecular mobility of polymers by disrupting the hydrogen bonding interaction between polymer chains ${ }^{18}$. Glycerol has been safely used in many industrial and pharmaceutical applications for over 100 years and is generally recognized for its low risk health effects ${ }^{19-22}$.

Given these premises, we investigated the effects of two glycerol-plasticized biomaterials (Alginate-Glycerol (HA-A-G) and Gelatin-Glycerol (Ge-G)), on a rat model of colonic anastomosis. The outcomes were further studied by means of CAM assay and in vitro tests on the different components of the biomaterials.

\section{Methods}

\section{Materials}

Sodium alginate from Laminaria hyperborea (Alginate Pronova UP LVG, relative molecular mass, $\mathrm{MW}, \sim 120.000$; fraction of guluronic $\mathrm{G}$ residues, $\mathrm{F}_{\mathrm{G}}=0.69$; fraction of guluronic diads, $\mathrm{F}_{\mathrm{GG}}=0.59$; number average of $\mathrm{G}$ residues in G-blocks, $\mathrm{N}_{\mathrm{G}}>1=16.3$ ) was kindly provided by Novamatrix/FMC Biopolymer (Sandvika, Norway). HA (MW 240.000, Phylcare Sodium Hyaluronate extra LW) was kindly provided by Sigea S.r.l. (Trieste, Italy). Calcium carbonate $\left(\mathrm{CaCO}_{3}\right)$, D-Gluconic acid $\delta$-lactone (GDL), glycerol, were purchased from Sigma Aldrich. The gelatin-based membrane (Willospon $囚$ ) was purchased from Will-Pharma, the Netherlands.

\section{Membrane preparation}

HA-A-G membranes were prepared according to the procedure described by some of the authors ${ }^{5}$. Briefly, alginate and HA were dissolved in deionized water (final concentration=15 g/l of each polysaccharide) and glycerol was added as a plasticizer (final concentration $=5 \% \mathrm{v} / \mathrm{v}$ ). Then, $\mathrm{CaCO}_{3}$ (final concentration of $\mathrm{Ca}^{2+}=20 \mathrm{mM}$ ) and GDL (final concentration=40 mM) were added to the mixture to enable the in situ gelation of the solution. After gelation, the membranes were obtained by freeze-drying. Membranes without the addition of glycerol (samples HA-A and A) were also prepared. The resulting freeze-dried materials were rehydrated with saline solution before application. The gelatin-glycerol (Ge-G) sample was prepared by soaking the gelatin-based membrane in $10 \mathrm{ml}$ of an aqueous solution of glycerol $(5 \% \mathrm{v} / \mathrm{v})$ for $1 \mathrm{~min}$, followed by freeze-drying. The gelatin-HA (Ge-HA) material was prepared by soaking the membrane in $10 \mathrm{ml}$ of a 
solution of HA (1.5\% w/v) for $1 \mathrm{~min}$, followed by freeze-drying. All the samples were sterilized by gamma radiation ( $25 \mathrm{kGy}$ ).

\section{Animals}

Sixty-six male Wistar rats with an average body weight of 250-300 grams were used. Animals were housed at the Central Animal Facilities of the Maastricht University, where they were provided ad libitum access to food and water, and were cared for according to local standards. Postoperatively, welfare assessment was performed twice daily using a standardized method and animals were given pain medication in case of discomfort. At 7 days follow-up or when humane endpoints were reached, animals were sacrificed. The experimental protocol complied with the Dutch Animal Experimental Act and was approved by the Animal Experimental Committee of Maastricht University Medical Center. This article followed ARRIVE guidelines for reporting the experiments. Supplementary information regarding the animal experiments can be found online.

\section{Study design}

\section{Experiment 1 ("leaking model")}

HA-A-G patch versus Ge-G versus control group in a rat model of colonic anastomotic leakage (4 interrupted sutures) to obtain an anastomotic leakage rate of $60-70 \%(n=30)$.

\section{Experiment 2 ("healing model")}

HA-A-G patch versus Ge-G versus control group in a rat model of colonic anastomosis (12 interrupted sutures, non-leaking model, $\mathrm{n}=36$ ).

\section{Surgical procedure}

All rats received $0.05 \mathrm{mg} / \mathrm{kg}$ buprenorphine s.c. as analgesic and were anesthetized using isoflurane (5\% induction, $2-2.5 \%$ maintenance). To acquire access to the abdominal cavity, a $5 \mathrm{~cm}$ craniocaudal midline incision of the skin and abdominal musculature was made in all experiments. The cecum was then identified and moved outside of the peritoneal cavity and onto sterile gauzes that were hydrated with sterile saline solution to prevent dehydration. Subsequently, the colon was transected two centimeters distal from the cecum and an end-to-end anastomosis was created using either 4 or 12 interrupted poly(propylene) 6/0 sutures (Prolene, Ethicon, Johnson \& Johnson) in experiment 1 and 2, respectively. The membranes were fixated around the anastomosis using sutures (poly(propylene) 6/0). In both experiment 1 and 2, rats assigned to control groups received no patch. After placement of the membrane, the intestines were repositioned and the abdomen 
was closed in two layers, a running suture for the muscle layer (Vicryl 4-0, Ethicon, Inc) and interrupted sutures for the skin (Monocryl 4-0, Ethicon, Inc).

\section{Macroscopic endpoints}

Anastomotic leakage was evaluated with the following scoring system: $0=$ no anastomotic leakage, $1=$ small abscess at the anastomotic site $\left(<1 \mathrm{~cm}^{3}\right), 2=$ large abscess at the anastomotic site $\left(>1 \mathrm{~cm}^{3}\right), 3=$ complete dehiscence with peritonitis and $4=$ death due to fecal peritonitis. Adhesions to the anastomotic site were assessed according to van der Ham et al. ${ }^{23}$ : $0=$ no adhesions, $1=$ minimal adhesions occurring mainly between the anastomosis and the omentum, 2=moderate adhesions occurring between the omentum and the anastomotic site and between the anastomosis and a loop of small bowel and 3 = severe and extensive adhesions, including abscess formation.

\section{Tissue and plasma preparation}

In anesthetized rats, the anastomotic site was dissected with a $0.5 \mathrm{~cm}$ margin at each site of the anastomosis. Tissue samples were cut in longitudinal direction and tissue was stretched and pinned onto a cork layer in order to secure a straight anastomotic line and improve quality of histological assessment prior to fixation in formalin.

\section{Histology and Immunohistochemistry}

Sections were deparaffinized in xylene and rehydrated in graded ethanol to distilled water and subsequently stained with standard hematoxylin-eosin staining and Ki67 immunohistochemistry. For the latter, endogenous peroxidase activity was blocked by incubating slides in $0.6 \%$ hydrogen peroxide/methanol for 15 minutes. Antigen retrieval was performed using target retrieval solution (Dako, Denmark) at $95^{\circ} \mathrm{C}$ for $20 \mathrm{~min}$. Non-specific antibody binding was blocked using 5\% fetal calf serum in PBS. Antibodies were Ki67 Clone Sp6 (Thermo Scientific, the Netherlands) and rabbit IgG biotine labelled (Dako, Denmark) as primary and secondary antibodies, respectively. Binding of primary antibody was visualized with 3,3'-diaminobenzidine-tetrahydrochloride-dihydrate (Sigma, St Louis, MO) and counterstained with hematoxylin. No staining was detected in slides incubated without primary antibody. An independent, experienced animal pathologist (MG) performed blinded histological assessment on the obtained tissue, scoring sections on inflammation, granulocyte influx and collagen deposition. Serosa thickness (in $\mu \mathrm{m}$ ) was measured using the free hand line selection tool in Image (Rasband, NIH, Maryland, USA). 


\section{Chorioallantoic membrane (CAM) Assay}

The biocompatibility of each patch component (alginate, hyaluronan, gelatin and glycerol) was tested by performing the chicken embryo chorioallantoic membrane (CAM) assay on patches with different compositions (Table 8.1). Samples HA-A-G and $\mathrm{Ge}-\mathrm{G}$ are the original patches tested in vivo. Samples HA-A and Ge are controls introduced to verify the effect of the removal of glycerol; patch $A$ is composed of only alginate, and was used to test its specific effect on CAM; patch Ge-HA, obtained by adding hyaluronan (at the same concentration of the HA-A-G patch) to the gelatin matrix, was studied to verify the effect of HA released from a different substrate. Pure sterile glycerol was also tested (sample G). For the assay, the fertilized eggs were disinfected (ethanol $70 \% \mathrm{v} / \mathrm{v}$ ) and placed in an incubator $\left(38^{\circ} \mathrm{C}, 60 \%\right.$ relative humidity). At day 3 of incubation, a window opening was performed at the blunt end of the egg, to select the living embryos. The window was covered with a polyethylene film glued with albumen, to avoid water loss and microbial contamination. At day 6 of incubation, the samples (round pieces of patches, diameter of $6 \mathrm{~mm}$, or $30 \mu \mathrm{l}$ of glycerol) were applied directly on the $\mathrm{CAM}^{24,25}$. Samples HA-A and A were hydrated with $30 \mu \mathrm{l}$ of saline solution before application.

Table 8.1 Components tested in the CAM assay.

\begin{tabular}{lll}
\hline Number & Sample & Composition \\
\hline 1 & HA-A-G & Patch of alginate with hyaluronan and glycerol \\
2 & Ge-G & Patch of gelatin with glycerol \\
3 & HA-A & Patch of alginate with hyaluronan \\
4 & Ge & Patch of gelatin \\
5 & A & Patch of alginate \\
6 & Ge-HA & Patch of gelatin with hyaluronan \\
7 & Ge-A & Patch of gelatin with alginate \\
8 & G & Pure glycerol \\
\hline
\end{tabular}

A system composed of a Leica WILD M32 stereomicroscope, equipped with a WILD PLAN $1 X$ lens, and connected to a Leica DFC 320 camera, was used to follow the effects of the samples on the CAM (at time points $t=24 \mathrm{~h}, 48 \mathrm{~h}$ ). A blinded evaluation was carried out by two independent experts, by considering the tissue damage and the inflammatory response, in particular vascular changes in the CAM such as hemorrhages, neoangiogenesis, and the occurrence of vessels devoid of blood flow (ghost vessels). Each sample has been assayed at least in 6 replicates.

\section{Cell culture}

To test the hypothesis that glycerol in the patch was harmful to or could activate the omentum leading to encapsulation of the patch, the effect of glycerol in four 
different concentrations $(1 \% \mathrm{v} / \mathrm{v}, 2 \% \mathrm{v} / \mathrm{v}, 4 \% \mathrm{v} / \mathrm{v}$ and $8 \% \mathrm{v} / \mathrm{v})$ on cell viability was tested on two sets of primary cells called human multipotent adipose derived stem cells (hMADS) isolated from human visceral adipose tissue. One obtained from a male lean donor (BMI 25.1) and the other from a male obese donor (BMI 39.6). Rats have a relatively large amount of fat tissue, especially scrotal fat, which is the reason why two different donorsets of hMADS were chosen. As a control, cells cultured in plain medium were considered. Cells were plated at a density of $2 \times 10^{3}$ cells $/ \mathrm{cm}^{2}$ and kept in proliferation medium (DMEM-HAM's F12 (Gibco, Blijswijk, the Netherlands)/10\%FBS (Bodinco BV, the Netherlands)/1xAnti-Anti (Gibco, Bleiswijk, the Netherlands)). Both cell lines were maintained at $37^{\circ} \mathrm{C}, 5 \% \mathrm{CO} 2$ in a humidified incubator as described previously ${ }^{26}$. After $24 \mathrm{~h}$, proliferation medium was replaced by proliferation medium supplemented with sterile glycerol (Sigma Alderich, the Netherlands) in increasing concentrations. Cells were trypsinized at day 3 and day 10 and the obtained cell suspensions were counted using trypan blue and a Bürker Türk counting chamber.

\section{Statistical analyses}

One-way ANOVA was used in case of continuous variables, with a Dunnett's Multiple Comparison Test as post-hoc analysis to compare groups versus the control group. A Kruskal-Wallis one-way analysis of variance was used in case of dichotomous variables. A $P$-value $\leq 0.05$ was considered statistically significant. All analyses were performed using IBM SPSS Statistics, version 21.0 for Mac (IBM SPSS, USA), while graphs were composed using GraphPad Prism, version 5.0a for Mac (GraphPad Software, USA).

\section{Results}

\section{Macroscopic examination}

Both in Experiment 1 and 2, the addition of the plasticized membranes around the anastomotic site caused adverse tissue reactions - which lead to higher anastomotic leakage rates compared to the control groups (Figure 8.1A). In Experiment 1 (leaking model), four rats of the experimental groups reached humane endpoints and were sacrificed before the end of follow-up. Inspection of the abdominal cavity following sacrifice confirmed that the 4 rats suffered from extensive fecal pollution; therefore the cause of death was stated to be sepsis as a result of fecal peritonitis. Remaining animals were sacrificed at follow-up day 7 . Control animals recovered readily from surgery and additional pain treatment was hardly necessary while membrane-receiving rats showed clear signs of discomfort, which resulted in a non-significant higher percentage weight loss (Figure 8.1B). 
Animals who had received a membrane showed formation of large abscesses and extensive adhesions, whilst control animals had significantly less signs of AL (Figure 8.2). In Experiment 2 (healing model), no animals died and the anastomosis healed properly in both the intervention and the control group. All membranes were evidently encapsulated; some cavities were filled with purulent or serosanguineous fluid. All encapsulation sites were next to the anastomotic site and did not involve the anastomosis. Adhesions were significantly more present in the membrane groups as compared with the control group, both in the leakage model and in the healing model (Figure 8.1C).
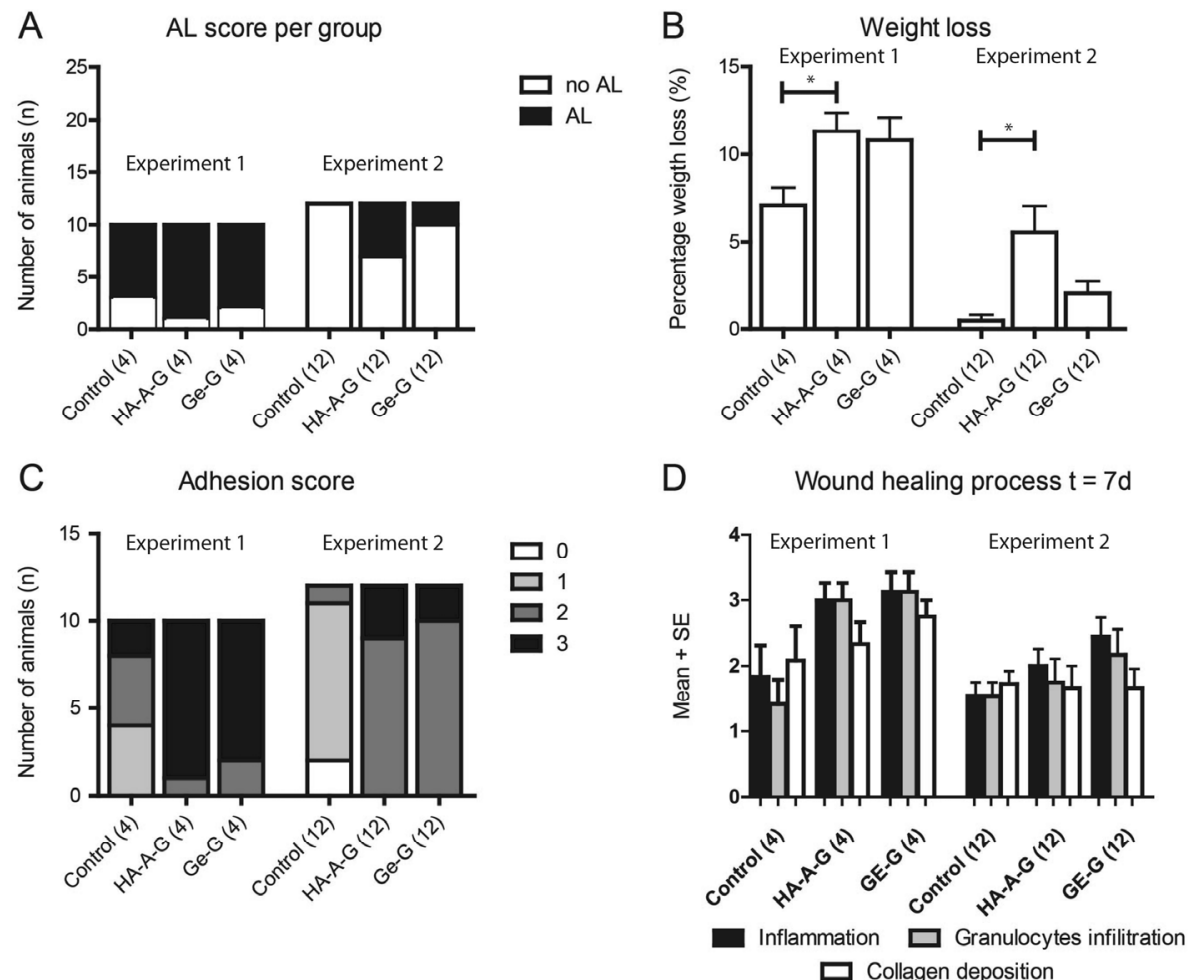

Figure 8.1 Anastomotic leakage rate were approximately $70 \%$ in the control group with a 4 -sutures anastomosis (A). Patches did not reduce anastomotic leakage rates, not in the healing model nor in the leakage model, but showed an increase in the amount of animals that suffered from anastomotic leakage. Weight loss was more severe in the leakage model (B), but also increased by adding a patch (HA-A-G vs control, $P<0.05$ ). In the sufficient anastomosis model, weight loss was significantly higher in the HA-A-G compared to the control group $(P<0.05)$. Severe adhesions with abscess formation were mostly found in the leakage group, but also in the sufficient anastomosis group in animals that received patches (C). No significant differences were found in terms of inflammation when obtained tissue was histologically evaluated (D). 


\section{Leakage model \\ (4 sutures)}

Healing model

(12 sutures)

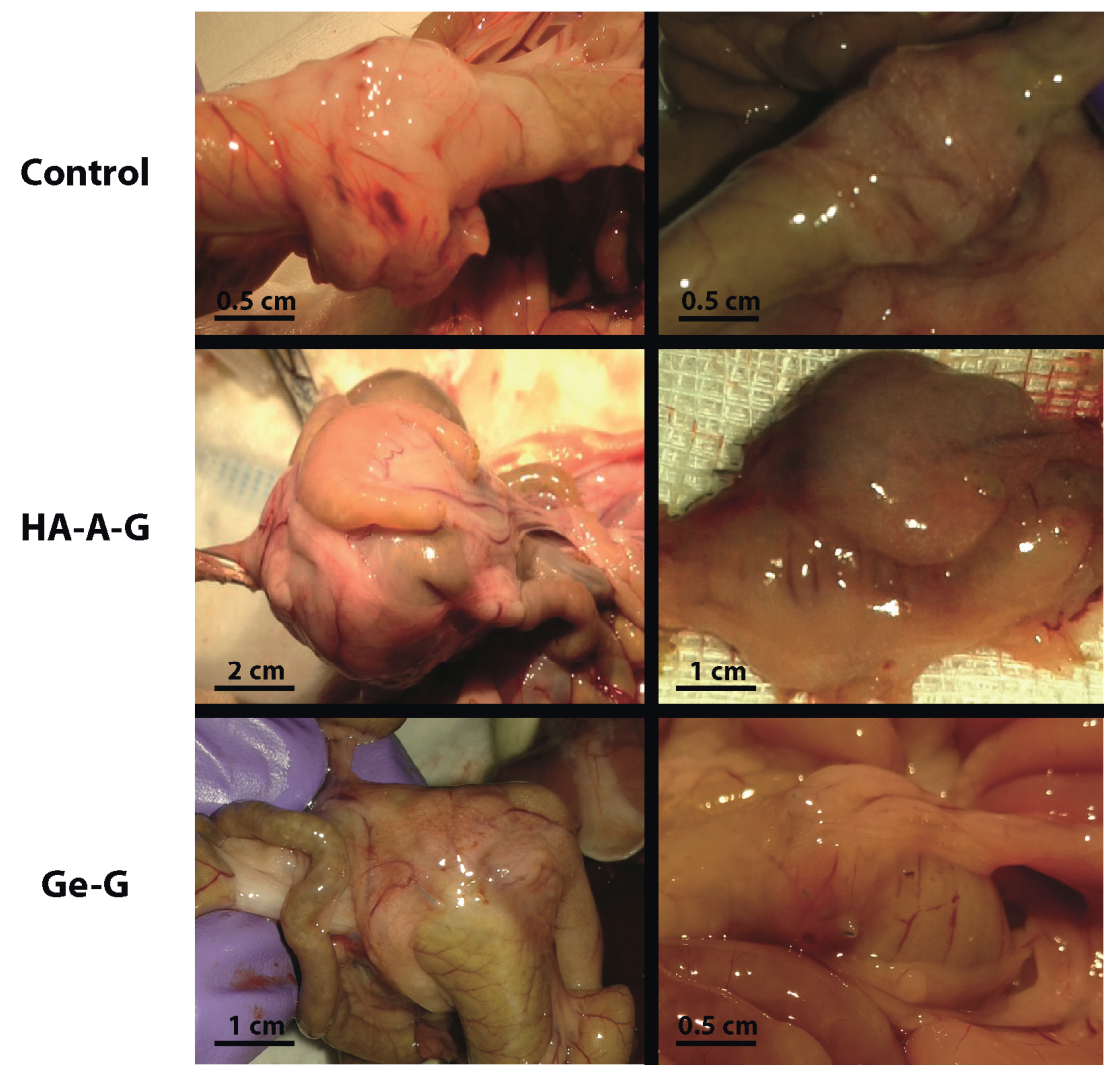

Figure 8.2 Macroscopic evaluation revealed encapsulation with large abscess formation in the leakage model, In the healing model, similar results were found, but here there were no evident abscesses, the cavities were not filled with purulent but serosanguineous fluid. In both experiments, adverse events were more severe in the HA-A-G group than in the Ge-G group. Control animals showed filmy adhesions to the anastomotic site.

\section{Histological assessment}

A trend in increase in inflammatory cells, such as granulocytes and macrophages was observed in all interventions groups compared to control animals with a significant difference in inflammation between control and Ge-G patches (Figure 8.1D, $P=0.03$ in the 4 sutures group and $P=0.05$ in the 12 sutures group). Furthermore, a large increase in serosa thickness was observed in the healing model (Figure 8.4) in the presence of the patches $(101 \pm 10.3 \mu \mathrm{m}$ and $92 \pm 7.8 \mu \mathrm{m}$ vs. 
$23 \pm 3.4 \mu \mathrm{m}, P<0.0001$, Figure $8.3 \mathrm{~A}$ ). In the leakage model this could not be analysed due to insufficient quality of the tissue.
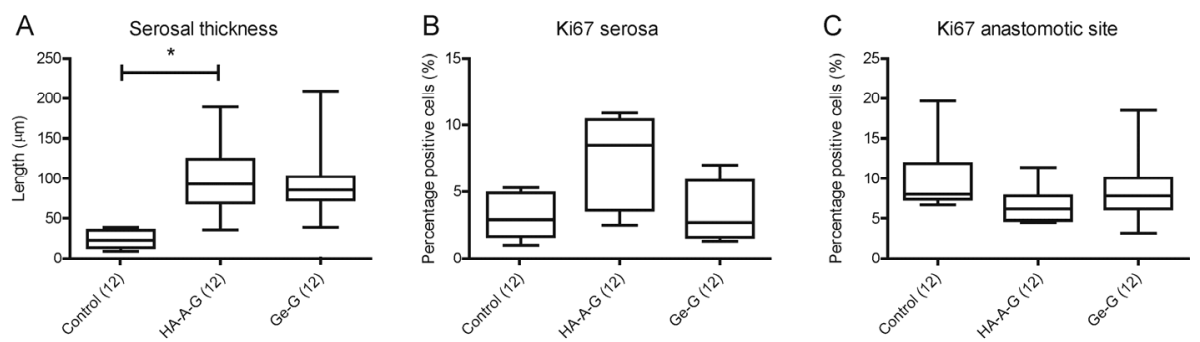

Figure 8.3 Serosal thickness was significantly increased in the presence of a patch (A). No significant differences were found in proliferation measured by Ki67 expression at the serosa (B) nor at the anastomotic site $(\mathrm{C})$.

\section{Immunohistochemistry}

To assess cell proliferation, the amount of Ki67 positive cells was counted. At the anastomotic site, no differences could be found in the percentage of Ki67+ cells between membrane (\%) and control groups (\%) $(P=0.12$, Figure 8.3B). However, when the focus was transferred to the serosal tissue, a trend towards a higher proliferation rate was observed in the presence of the HA-A-G membrane $(7.3 \pm 1.6 \%)$ compared with control tissue $(3.2 \pm 0.8 \%)(P=0.056$, Figure $8.3 \mathrm{C})$.

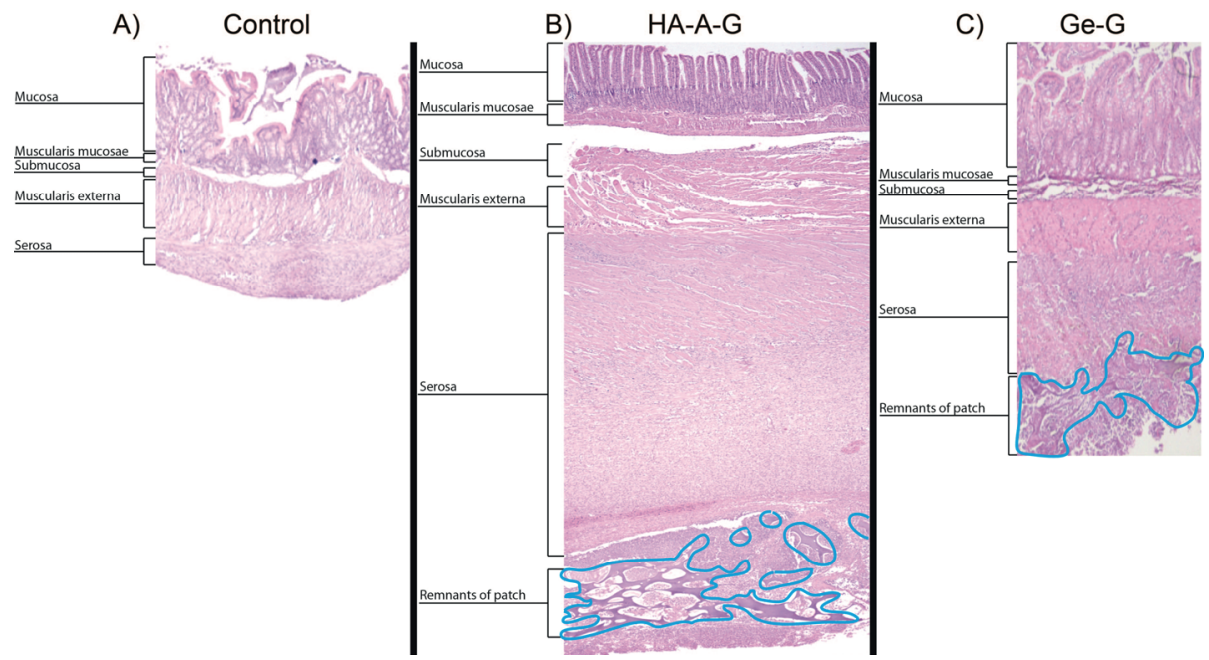

Figure 8.4 Overview of the different histological layers in the intestinal wall. The presence of a patch $(B+C)$ caused an increase in serosa thickness compared to control tissue $(A)$. 


\section{Glycerol showed reduced biocompatibility in CAM Assay}

Since the in vivo studies, unexpectedly, pointed out adverse-tissue reactions in the presence of the plasticized membranes, a further biological insight was tackled to elucidate the cause of such behaviour. To this end, the chorioallantoic membrane (CAM) assay was employed to evaluate the biological response to the materials. The CAM assay is a test that has shown a renewed interest in the biomaterial field $25,27,28$ since it can be used to easily investigate and evaluate tissue reaction and biocompatibility of materials, both in solid and liquid form.

The CAM photographs obtained at time points $\mathrm{t}=24 \mathrm{~h}, 48 \mathrm{~h}$ after the deposition of the samples are reported in Figure 8.5. For the samples HA-A-G and Ge-G (Figure 8.5 , rows $a$ and $b$ ) tissue injury, characterized by vessel damage and bleeding, was observed. This reaction corresponds, generally, to toxicity and/or inflammatory responses in vivo and, in the case of biomaterials, it is associated to low biocompatibility. When pure glycerol was tested on the CAM, it also induced a clear adverse reaction: tissue damage - with hemorrhagic events after sample placement on the CAM - was observed, combined with an increased mortality. After $24 \mathrm{~h}$ from the treatment, changes in the structure of the CAM (thickening of the membrane, formation of a white callus, and presence of red blood cells) were observed, as a consequence of the chemical insult (Figure 8.5, row c). On the contrary, all the patches without glycerol (Figure 8.5, rows d-g) resulted completely biocompatible. Indeed, changes over time were associated to the normal membrane development and growth of blood vessels, without any significant adverse reaction.

\section{Cell culture revealed a dose-response effect of glycerol on pre-adipocytes}

Next, hMADS were treated with increasing glycerol concentration $(1-8 \% \mathrm{v} / \mathrm{v})$ to investigate cell viability. All cells were affected by the introduction of glycerol, however, hMADS derived from lean patients showed better recovering properties compared with obese hMADS (Figure 8.6). Overall, cells seemed to recover quicker and when only $1 \%$ glycerol was added, cells reached the same level of confluence as the control group. In both experimental setups, $8 \% \mathrm{v} / \mathrm{v}$ glycerol was toxic and caused cell death, shortly after adding glycerol to the plate. Both the $2 \% \mathrm{v} / \mathrm{v}$ and $4 \% \mathrm{v} / \mathrm{v}$ groups showed debris and dead cells in the supernatant, but to a lesser extent than the $8 \% \mathrm{v} / \mathrm{v}$. 

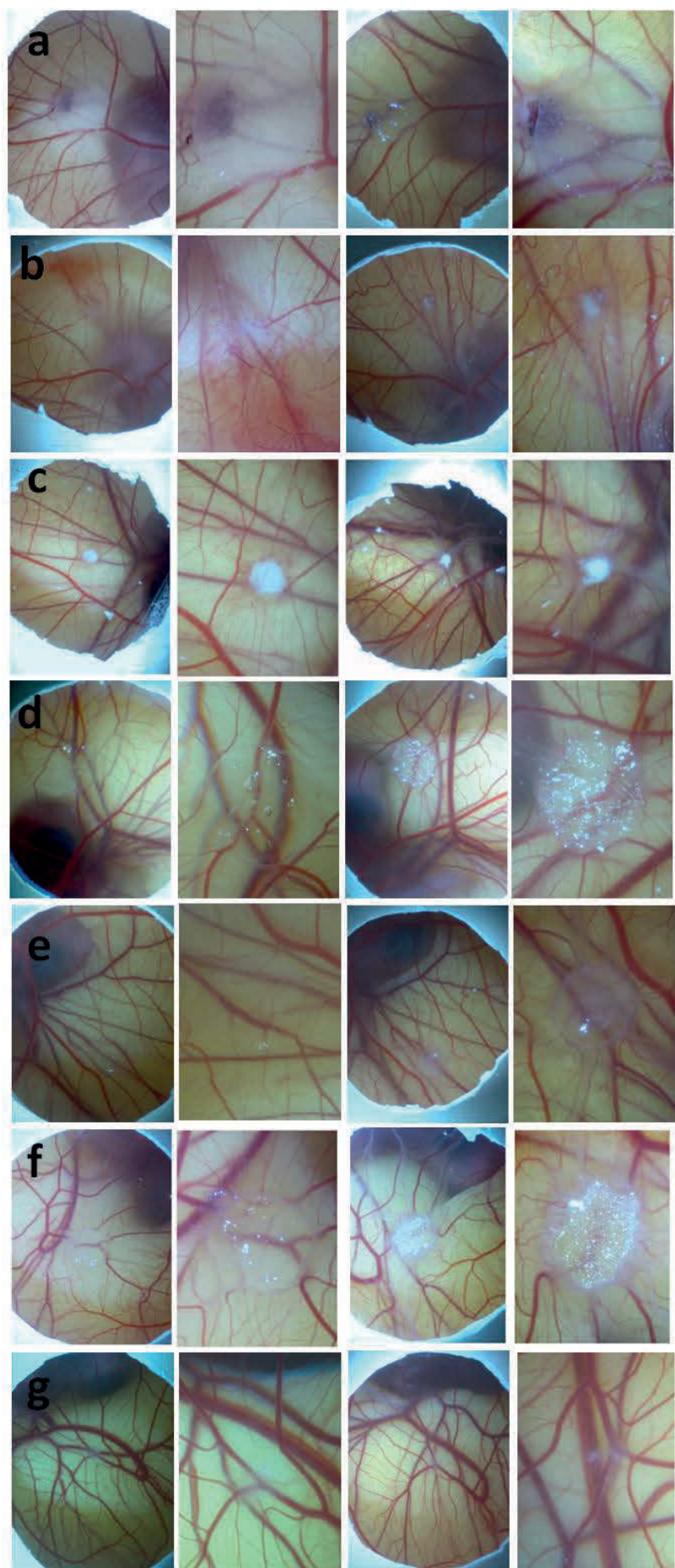
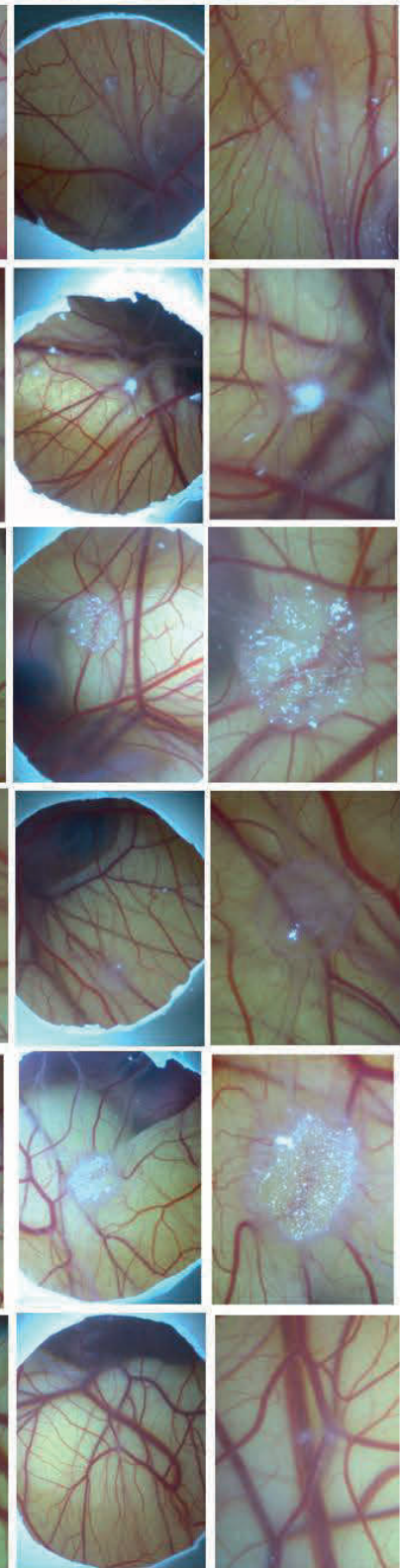

Figure 8.5 CAM images at $12 \mathrm{x}$

magnification taken from the top of the egg after deposition of the samples. Effect of the patches on chicken embryos after 0, 24 and $48 \mathrm{~h}$ from deposition of samples HA-A-G (a), Ge-G (b), HA-A (c), Ge (d), A (e), Ge-HA (f). Effect of pure glycerol on chicken embryo after 0 and $20 \mathrm{~min}$ from deposition (g) 
A Effect of glycerol concentration on obese hMADS
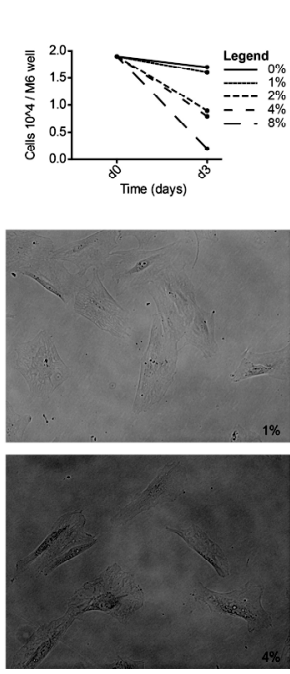
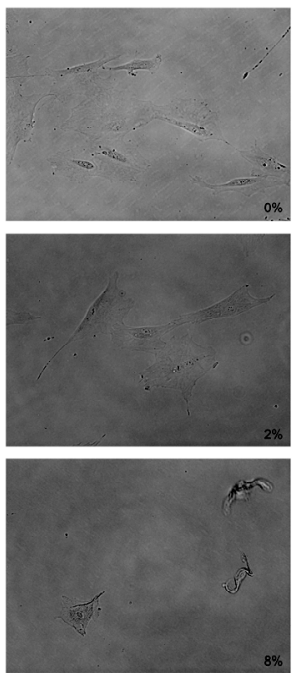

Effect of glycerol concentration on lean hMADS
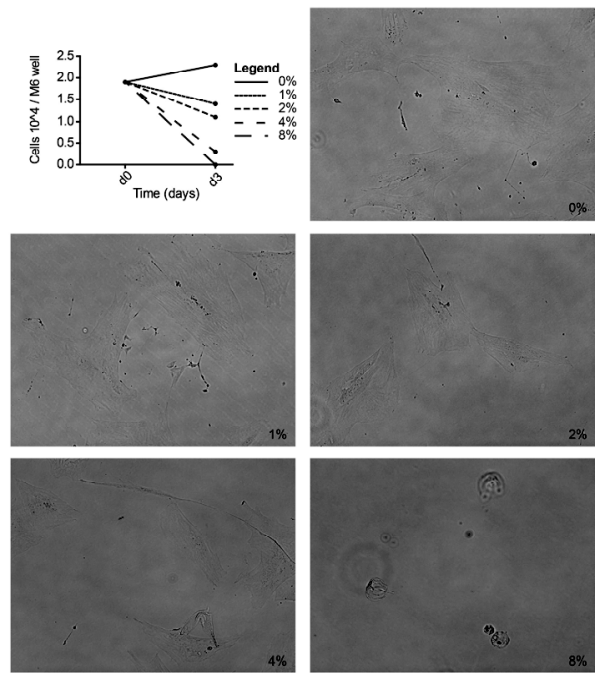

Figure 8.6 hMADS were exposed to glycerol and cell viability was measured by counting cells/well. Both adipose-derived stem cells from an obese person (A) and from a lean person (B) showed complete cell-death after $8 \% \mathrm{v} / \mathrm{v}$ glycerol in the medium and severe damage from both $2 \% \mathrm{v} / \mathrm{v}$ and $4 \% \mathrm{v} / \mathrm{v}$.

\section{Discussion}

Several attempts have been made to seal off the anastomotic site in order to prevent anastomotic leakage in patients undergoing colorectal surgery. Previous studies have shown that the use of membranes around the anastomosis can cause stenosis or bowel obstruction ${ }^{29,30}$ making it crucial that the patches be flexible and do not interfere with intestinal motility. For that reason, glycerol was added as a plasticizer. Glycerol is a commonly used plasticizer, even in medical and pharmaceutical applications and is used to increase the process-ability, flexibility and durability of biomaterials ${ }^{17}$. The addition of glycerol improves the characteristics of the material, making it easy to use and suitable for a broad range of applications ${ }^{31}$.

In this study, two membranes based on hyaluronan/alginate and porcinederived gelatin plasticized with glycerol were tested in animal models of colonic anastomoses to promote anastomotic healing. In both experiments (leaking and healing model), the plasticized membranes did not reduce AL rates, however, rats receiving membranes experienced more discomfort, showed encapsulation/ induration of patch tissue and had a decreased survival compared to the control 
groups. Additional CAM assay and cell-culture analysis revealed glycerol being the cause of the side effects, possibly due to its pro-inflammatory effect and cytotoxic effect.

Morphologically, a clear encapsulation of the patch with indurated tissue was observed in both models. This tissue remodeling has been described by Wilkosz and colleagues during adhesion formation ${ }^{32}$. In their experiment, collagen deposition and fibrotic foci were predominantly present at day 7 , an observation that could also be found in this study. A possible explanation for the observed morphological changes is that glycerol in the patch can activate the omentum which lead to rapid extension and expansion, resulting in encapsulation of the patch as if to protect the adjacent internal organs from contact with it. This biological response can prevent the potential beneficial effects from the patch on the healing process $^{33}$. This was evident in the healing model, where no leakage occurred, but even the construction of a sufficient anastomosis causes serosal damage due to suture placement. Serosal healing involves implantation, proliferation and incorporation of free-floating mesothelial cells into the regenerating mesothelium ${ }^{34}$ which may explain the increase of serosa thickness in histological transections. It is known that adhesions can occur postoperatively due to injury of the mesothelium which lines the peritoneal cavity ${ }^{32,35}$. If encapsulation occurs simultaneously with $\mathrm{AL}$, an abscess can develop with extensive adhesions, which was observed in the leakage model of this study. To evaluate if the omentum reacts to glycerol, we performed cell-culture with hMADS from human omentum in the presence of different concentrations of glycerol and noticed a severe decrease in the amount of viable cells when adding $>2 \% \mathrm{v} / \mathrm{v}$ glycerol to the medium.

The latter observation suggests a dose-dependent effect of glycerol. Interestingly, it has been demonstrated that high concentrations of glycerol can cause damage to cell membranes ${ }^{36}$. Garcia and colleagues suggested that this damage could be related to an osmotic effect ${ }^{36}$. Indeed, glycerol is often used in the clinical setting to treat diarrhea since it works as an osmotic diuretic. Therefore, extracellular hyperosmolarity might also be a potential reason for the unexpected results. It has been described that inflammation occurs when cells are exposed to high osmolarities (>300 mOsm) ${ }^{37}$. Epithelial cells, such as normal colorectal epithelial cells can induce proinflammatory cytokine secretion through hyperosmolarity ${ }^{37}$.

The glycerol-containing membranes caused adverse reactions in rats and the CAM assay showed clear negative effects of glycerol. This was also found in the cell culture experiment, in which the percentage of glycerol had an obvious harmful effect on adipose-derived stem cells. Concerns that glycerol may have harmful 
effects on different cell types and species were already raised in the 80s by some research groups ${ }^{38,39}$. Nevertheless, glycerol has been a frequently used plasticizer in several commercial biomaterials ${ }^{31}$. For example, it has been demonstrated that high concentrations of glycerol ( $>75 \%)$ in a commercially available bone matrix could lead to myonecrosis and rhabdomyolysis which resulted acute renal failure in experimental animals ${ }^{40}$. Despite the obvious differences between animal models and the clinical setting, the combination of detrimental effects observed both in vitro (on human cells) and in vivo, together with some evidence from literature, makes us advocate taking caution when selecting glycerol as a plasticizer.

Overall, this paper pointed out that, despite preliminary indications of materials' biocompatibility in vitro ${ }^{5}$, unexpected adverse reactions could take place in real surgical models: the CAM assay might provide a useful mean to predict possible adverse effects of single components of the biomaterials.

From this study, we can conclude that glycerol can have a pro-inflammatory effect in vivo and that it reduces viability of human multipotent adipose-derived stem cells (hMADS). The widespread use of glycerol as a plasticizer should be limited to only cases in which it is $100 \%$ certain that its use is safe and causes no potential risks. In the future, caution should be taken when selecting glycerol as plasticizer in biomaterials designed for intra-abdominal use. 


\section{References}

1. McArdle CS, McMillan DC, Hole DJ. Impact of anastomotic leakage on long-term survival of patients undergoing curative resection for colorectal cancer. Br J Surg. 2005;92(9):1150-1154.

2. Khan AA, Wheeler JMD, Cunningham C, George B, Kettlewell M, Mortensen NJM. The management and outcome of anastomotic leaks in colorectal surgery. Colorectal Dis. 2008;10(6):587-592.

3. Bosmans JWAM, Jongen ACHM, Bouvy ND, Derikx JPM. Colorectal anastomotic healing: why the biological processes that lead to anastomotic leakage should be revealed prior to conducting intervention studies. BMC Gastroenterol. 2015;15(1):180.

4. Dornish M, Donati I, Bouvy N. Anastomoseal-Biopolymeric Patches for the Treatment of Colorectal Anastomosis. Tissue Engineering Part A, 2014;2:S78.

5. Travan A, Scognamiglio F, Borgogna M, Marsich E, Donati I, Tarusha L, et al. Hyaluronan delivery by polymer demixing in polysaccharide-based hydrogels and membranes for biomedical applications. Carbohydr Polym. 2016 Oct 5;150:408-418

6. Sundaram CP, Keenan AC. Evolution of hemostatic agents in surgical practice. Indian J Urol. 2010;26(3):374-378.

7. Aya KL, Stern R. Hyaluronan in wound healing: rediscovering a major player. Wound Repair Regen. 2014;22(5):579-93.

8. Price RD, Berry MG, Navsaria HA. Hyaluronic acid: the scientific and clinical evidence. JPRAS 2007;60(10):1110-1119.

9. Wiegand C, Heinze T, Hipler UC. Comparative in vitro study on cytotoxicity, antimicrobial activity, and binding capacity for pathophysiological factors in chronic wounds of alginate and silver-containing alginate. Wound Repair Regen. 2009;17(4):511-521.

10. Augst AD, Kong HJ, Mooney DJ. Alginate Hydrogels as Biomaterials. Macromol Biosci. 2006;6(8):623-33.

11. Travan A, Fiorentino S, Grassi M, Borgogna M, Marsich E, Paoletti S, et al. Rheology of mixed alginate-hyaluronan aqueous solutions. Int J Biol Macromol. 2015;78:363-369.

12. Geremia I, Borgogna M, Travan A, Marsich E, Paoletti S, Donati I. Determination of the composition for binary mixtures of polyanions: the case of mixed solutions of alginate and hyaluronan. Biomacromolecules. 2014;15(3):1069-1073.

13. Scognamiglio F, Travan A, Borgogna M, Donati I, Marsich E, Bosmans J, et al. Enhanced bioadhesivity of dopamine-functionalized polysaccharidic membranes for general surgery applications. Acta Biomater. 2016:1-20.

14. Rullan PP, Vallbona C, Rullan JM, Mansbridge JN, Morhenn VB. Use of gelatin sponges in Mohs micrographic surgery defects and staged melanoma excisions: a novel approach to secondary wound healing. J Drugs Dermatol. 2011;10(1):68-73.

15. Pantelis D, Beissel A, Kahl P, Wehner S, Vilz TO, Kalff JC. The effect of sealing with a fixed combination of collagen matrix-bound coagulation factors on the healing of colonic anastomoses in experimental high-risk mice models. Langenbecks Arch Surg. 2010;395(8):1039-1048.

16. Nordentoft T, Pommergaard HC, Rosenberg J, Achiam MP. Fibrin Glue Does Not Improve Healing of Gastrointestinal Anastomoses: A Systematic Review. Eur Surg Res. 2015;54(1-2):1-13.

17. Vieira MGA, da Silva MA, Santos dos LO, Beppu MM. Natural-based plasticizers and biopolymer films: A review. Eur Polym J. 2011;47(3):254-263.

18. Cervera MF, Karjalainen M, Airaksinen S, Rantanen J, Krogars K, Heinämäki J, et al. Physical stability and moisture sorption of aqueous chitosan-amylose starch films plasticized with polyols. Eur J Pharm Biopharm. 2004;58(1):69-76.

19. Migneco F, Huang Y-C, Birla RK, Hollister SJ. Poly(glycerol-dodecanoate), a biodegradable polyester for medical devices and tissue engineering scaffolds. Biomaterials. 2009;30(33):6479-6484.

20. Morrison LR. 'Glycerol' in Kirk-Othmer Encyclopedia of Chemical Technology. 2000. DOI: 10.1002/0471238961.0712250313151818.a01.

21. Sundback CA, McFadden J, Hart A, Kulig KM, Wieland AM, Pereira MJN, et al. Behavior of poly(glycerol sebacate) plugs in chronic tympanic membrane perforations. J Biomed Mater Res Part B Appl Biomater. 2012;100(7):1943-1954. 
22. Sundback CA, Shyu JY, Wang Y, Faquin WC, Langer RS, Vacanti JP, et al. Biocompatibility analysis of poly(glycerol sebacate) as a nerve guide material. Biomaterials. 2005;26(27):5454-5464.

23. van der Ham AC, Kort WJ, Weijma IM, van den Ingh HF, Jeekel J. Effect of fibrin sealant on the healing colonic anastomosis in the rat. Br J Surg. 1991;78(1):49-53.

24. Ribatti D, Vacca A, Roncali L, Dammacco F. The chick embryo chorioallantoic membrane as a model for in vivo research on angiogenesis. Int J Dev Biol. 1996;40(6):1189-1197.

25. Schoubben A, Blasi P, Marenzoni ML, Barberini L, Giovagnoli S, Cirotto C, et al. Capreomycin supergenerics for pulmonary tuberculosis treatment: Preparation, in vitro, and in vivo characterization. Eur J Pharm Biopharm. 2013;83(3):388-395.

26. Jocken JWE, Goossens GH, Popeijus H, Essers Y, Hoebers N, Blaak EE. Contribution of lipase deficiency to mitochondrial dysfunction and insulin resistance in hMADS adipocytes. Int J Obes (Lond). 2015 Oct 16. DOI: 10.1038/ijo.2015.211201524.

27. Saw CLL, Heng PWS, Liew CV. Chick chorioallantoic membrane as an in situ biological membrane for pharmaceutical formulation development: a review. Drug Dev Ind Pharm. 2008;34(11): 1168-1177.

28. Vargas A, Zeisser-Labouèbe M, Lange N, Gurny R, Delie F. The chick embryo and its chorioallantoic membrane (CAM) for the in vivo evaluation of drug delivery systems. Adv Drug Deliv Rev. 2007;59(11):1162-1176.

29. Schreinemacher MH, Bloemen JG, Heijden SJ, Gijbels MJ, Dejong CH, Bouvy ND. Collagen fleeces do not improve colonic anastomotic strength but increase bowel obstructions in an experimental rat model. Int J Colorectal Dis. 2011;26(6):729-735

30. Bae K-B, Kim S-H, Jung S-J, Hong K-H. Cyanoacrylate for colonic anastomosis; is it safe? Int J Colorectal Dis 2010;25(5):601-606.

31. Snejdrova E, Dittrich M. Pharmaceutically Used Plasticizers. In: Recent Advances in Plasticizers. InTech; 2012. DOI: $10.5772 / 2228$

32. Wilkosz S, Epstein J, de Giorgio Miller A, McLean W, Ireland G, Herrick S. Remodelling of adipose tissue during experimental omental adhesion formation. Br J Surg. 2008;95(3):387-396.

33. Litbarg NO, Gudehithlu KP, Sethupathi P, Arruda JAL, Dunea G, Singh AK. Activated omentum becomes rich in factors that promote healing and tissue regeneration. Cell Tissue Res. 2007;328(3):487-497.

34. Foley-Comer AJ, Herrick SE, Al-Mishlab T, Prêle CM, Laurent GJ, Mutsaers SE. Evidence for incorporation of free-floating mesothelial cells as a mechanism of serosal healing. J Cell Sci. 2002;115:1383-1389.

35. Beyene RT, Kavalukas SL, Barbul A. Intra-abdominal adhesions: Anatomy, physiology, pathophysiology, and treatment. Curr Probl Surg. 2015;52(7):271-319.

36. Macías García B, Ortega Ferrusola C, Aparicio IM, Miró-Morán A, Morillo Rodriguez A, Gallardo Bolaños JM, et al. Toxicity of glycerol for the stallion spermatozoa: Effects on membrane integrity and cytoskeleton, lipid peroxidation and mitochondrial membrane potential. Theriogenology. 2012;77(7):1280-1289.

37. Schwartz L, Guais A, Pooya M. Is inflammation a consequence of extracellular hyperosmolarity. J Inflamm (Lond). 2009;23(6):21. .

38. Armitage WJ, Mazur P. Toxic and osmotic effects of glycerol on human granulocytes. Am J Physiol. 1984;247:C382-389.

39. Rengachary SS, Watanabe IS, Singer P, Bopp WJ. Effect of Glycerol on Peripheral Nerve: An Experimental Study. Neurosurgery. 1983;13(6):681.

40. Wang JC, Kanim LE, Nagakawa IS, Yamane BH, Vinters HV, Dawson EG. Dose-dependent toxicity of a commercially available demineralized bone matrix material. Spine. 2001;26(13):1429-1436. 


\section{S 8.1 Supplemental information according to ARRIVE guidelines}

\section{Ethical statement}

- The experimental protocol complied with the Dutch Animal Experimental Act and was approved by the Animal Experimental Committee of Maastricht University Medical Center. Protocols for institutional animal use and care guidelines were followed (permit DEC 2012-055).

\section{Study design}

- In total there were 55 rats used in this study. We used 11 rats per experimental group, and 5 groups.

\section{Experimental procedures}

- $\quad$ For surgical procedure, see manuscript. This was carried out in the rat operating room of the animal facility of Maastricht University under semisterile conditions.

\section{Experimental animals}

- Male Wistar rats (RccHan:WIST) were purchased from Harlan laboratories, the Netherlands. Rats had an average weight of $307 \mathrm{~g}$ on the day of surgery (SD 16.7).

- We have chosen for male rats, since it is known that female estrogens and androgens have an important influence on wound-healing (Ashcroft GS, et al. Estrogen modulates cutaneous wound healing by downregulating macrophage migration inhibitory factor. The Journal of clinical investigation. 2003, 111:1309-1318).

\section{Housing and husbandry}

- An acclimatization period of one week was observed prior to the start of the experiment.

- $\quad$ Rats were kept under standard conditions and were provided with food and water ad libitum. Rats were housed 2 animals per cage. The general health of rats was monitored several times per week for signs of inflammation and animals were weighed once per week. During the experiment animals were weighed daily and scored for discomfort twice daily (every morning and every evening). In case of discomfort, additional pain treatment was administered by giving buprenorphine $0.05 \mathrm{mg} / \mathrm{kg}$ s.c.

- Discomfort was scored using a standard scheme (zie below). Humane endpoints were defined according to Roughan \& Flecknell (Roughan, J. V. \& 
Flecknell, P. A. Behavioural effects of laparotomy and analgesic effects of ketoprofen and carprofen in rats. Pain 90, 65-74 (2001).

- Humane endpoints are defined as:

○ Significant weight loss $>20 \%$

- Fever (temperature is only measured on indication)

- Tachypnoe

- Significant differences in behavior:

- Lethargia

- Twitching: random spasms of the muscles, can be seen when animals are asleep or inactive in huddled up position.

- Walking: unable to stand on four legs, wobbly walk

- Huddled up posture: showing a concave abdominal side. Can be seen when walking/sitting.

- Signs of severe dehydration

- Severe diarrhea

- Severely inflammed surgical wound

$\circ$ Cachexia

Allocating animals to experimental groups

- Animals were randomized by means of throwing a dice. Groups were divided into 1-5 (see table 1). For each animal the dice was thrown and the rat received the assigned treatment. Whenever 6 was thrown, the dice was rolled again.

\section{Numbers analysed}

- All analyses were performed according to an intention-to-treat analysis.

However, since there were adverse events and deaths prior to follow-up, for $\mathrm{ABP}$ and histological assessment these animals were not taken into account. 
Welfare scoring rat model

\begin{tabular}{|c|c|c|c|c|}
\hline \multicolumn{5}{|l|}{ Description } \\
\hline & 0 & 1 & 2 & 3 \\
\hline Activity & Normal & Isolated, less active & Inactive & $\begin{array}{l}\text { Somnulent, stupor, } \\
\text { coma, lifeless }\end{array}$ \\
\hline Behavior & Normal & $\begin{array}{l}\text { Back arching, } \\
\text { twitching, shivering } \\
\text { Once } / 10 \mathrm{~min}\end{array}$ & $\begin{array}{l}\text { Back arching, twitching, } \\
\text { shivering }\end{array}$ & $\begin{array}{l}\text { Stereotype } \\
\text { behavior, auto } \\
\text { mutilation, } \\
\text { aggressive behavior }\end{array}$ \\
\hline Gait & Normal & $\begin{array}{l}\text { Mildly } \\
\text { uncoordinated/ } \\
\text { Abnormality }\end{array}$ & $\begin{array}{l}\text { uncordinated walking on } \\
\text { toes, limping }\end{array}$ & $\begin{array}{l}\text { Paralysis, limp, } \\
\text { convulsions, tremor. }\end{array}$ \\
\hline Posture & Normal & $\begin{array}{l}\text { Huddled up, } \\
\text { stretching }\end{array}$ & Imbalance, twitching & Fall over, circle \\
\hline $\begin{array}{l}\text { Physical } \\
\text { condition }\end{array}$ & Normal & $\mathrm{BC} 2=$ condition & BC5 = obese & $\begin{array}{l}\text { BC1 = emaciated } \\
\text { BC6=extreme obese }\end{array}$ \\
\hline Fur/skin & Normal & $\begin{array}{l}\text { Dry, rough, not shiny } \\
\text { anymore }\end{array}$ & $\begin{array}{l}\text { Piloerection, small wounds, } \\
\text { porfyrie, dry white skin }\end{array}$ & $\begin{array}{l}\text { Red/black skin, } \\
\text { inflammation, } \\
\text { wounds, loss of fur }\end{array}$ \\
\hline Hydratation & Normal & Loss of skin elasticity & Reduced skin turgor & $\begin{array}{l}\text { Severly reduced } \\
\text { turgor + sunken } \\
\text { eyes }\end{array}$ \\
\hline Breathing & Normal & Fast and superficial & $\begin{array}{l}\text { Fast abdominal breathing + } \\
\text { audible breathing }\end{array}$ & $\begin{array}{l}\text { Respiratory } \\
\text { problems, cyanosis, } \\
\text { breathing with open } \\
\text { mouth }\end{array}$ \\
\hline Faeces/urine & Normal & Moist faeces, polyurie & Diarrhea, abnormal urine & $\begin{array}{l}\text { Uncontrolled } \\
\text { diarree, bloody } \\
\text { stool, obstipation, } \\
\text { hematuria }\end{array}$ \\
\hline Surgical wound & $\begin{array}{l}\text { Normal } \\
\text { healing }\end{array}$ & $\begin{array}{l}\text { Sutures intact, slighty } \\
\text { red/bloody }\end{array}$ & $\begin{array}{l}\text { Dehiscence of wound, } \\
\text { sutures open, fluid secretion }\end{array}$ & $\begin{array}{l}\text { Severe bleeding, } \\
\text { wound open, severe } \\
\text { redness, necrosis }\end{array}$ \\
\hline Edema & Normal & $\begin{array}{l}\text { Mild abnormal fluid } \\
\text { collections, swollen } \\
\text { appearance }\end{array}$ & $\begin{array}{l}\text { Abnormal large abnormal } \\
\text { fluid collections, ascites }\end{array}$ & $\begin{array}{l}\text { Severe large } \\
\text { abnormal fluid } \\
\text { collections }\end{array}$ \\
\hline Necrosis & Normal & Dark skin colouring & $\begin{array}{l}\text { Small dark/black spots, } \\
\text { burning wounds, blisters }\end{array}$ & $\begin{array}{l}\text { Big black spots, } \\
\text { crusts }\end{array}$ \\
\hline
\end{tabular}




\section{CHAPTER 9}

\section{A new slow-resorbable Poly(1,3-trimethylene carbonate) film provides safe and effective adhesion reduction after major abdominal surgery in a rat model}

Joanna W.A.M. Bosmans*, Ruben R.M. Vogels*,

Kevin W.Y. van Barneveld, Vincent Verdoold Marion J.J. Gijbels, Jos Penders, Stephanie O. Breukink, Gerard Beets, Dirk W. Grijpma, Nicole D. Bouvy

* Both autors contributed equally to this manuscript Surgery 2015;157(6):1113-20 


\section{Abstract}

\section{Introduction}

Postoperative adhesions remain a major clinical problem following abdominal surgery. We evaluated the efficacy of a new Poly(trimethylene Carbonate) (PTMC) film as an antiadhesive material. In many abdominal operations there is an increased risk of faecal contamination; therefore the safety of this film was studied in two additional animal models.

\section{Methods}

A validated rat adhesion model with peritoneal ischemic buttons was used to compare the new PTMC film with a Hyaluronate Carboxymethylcellulose (HA-CMC) sheet, Icodextrin solution, and a control group. Primary endpoint was occurrence of adhesions at the ischemic buttons after 14 days in 44 rats ( $n=11$ per group). To evaluate safety of the film, both an anastomotic leakage model and a cecal ligation and puncture model were used. KruskalWallis tests with subsequent Mann-Whitney tests were used to detect differences between groups. $P$-values $<0.008$ were considered statistically significant.

\section{Results}

PTMC film showed a significant reduction in the amount of adhesions (median: 0.5 buttons), when compared to control group (median: 4 buttons, $P<0.001$ ) and Icodextrin group (median: $4.5, P<0.001$ ). The amount of adhesions was similar to the HA-CMC group (median: 2, $P=0.04$ ). The presence of the film did not increase the risk of anastomotic leakage or bacterial growth in a contaminated environment.

\section{Conclusions}

The presence of a PTMC film leads to a significant reduction in the amount of adhesions after 14 days in an ischemic button rat model. Furthermore, this film can be safely used, even in complex abdominal operations with an increased risk of fecal contamination. 


\section{Introduction}

Adhesion formation after abdominal surgery is a major clinical problem, with an incidence of over $93 \%$ of all patients undergoing a laparotomy ${ }^{1-3}$. These postoperative adhesions are associated with many short- and long-term postoperative complications and lead to readmission of one in three patients within the first ten years ${ }^{2-4}$.

Unfortunately, the appearance of adhesion formation still is an underestimated clinical problem ${ }^{5-7}$. Many different materials have been developed for the prevention of postoperative adhesions ${ }^{8-10}$. Of these materials mainly the "barrier type" materials, like Hyaluronate Carboxymethylcellulose (HA-CMC) (Seprafilm ${ }^{\circledR}$ ) have been found to be effective in reducing the amount of severe adhesions $8,11,12$. Results on "liquid-based" adhesion prevention, such as intraperitoneal administration of Icodextrin (Adept ${ }^{\circledR}$ ), are still inconclusive ${ }^{10,12,13}$.

Aforementioned barrier materials are believed to work by providing an inert and inactive barrier between tissues to reduce adhesion formation without providing bioactive properties, allowing the peritoneum to heal, while inducing minimal foreign body reaction ${ }^{14,15}$. Although these materials show significant reduction in amount of adhesions, we believe these results can be improved by using a more stable barrier material. This could be achieved by using poly(trimethylene carbonate) (PTMC), an inert, slow degrading polymer with excellent biocompatibility ${ }^{16}$. In addition, it was shown that the polymer is phagocytised by macrophages and that at 12 weeks PTMC is degraded while only a small area of inflammatory cells could be observed at the site of implantation ${ }^{16-17}$. Unlike other materials that can be used to produce membrane, such as collagen, this polymer degrades through surface degradation, allowing it to retain its mechanical characteristics throughout the degradation process ${ }^{16-18}$. Furthermore, in bone regeneration studies PTMC promoted bone healing, without leading to osseous depositions inside the film due to solid composition, in contrast to collagenous membranes ${ }^{17}$. This lead to the hypothesis that PTMC could provide an adequate barrier against postoperative adhesions when placed intraperitoneally.

To evaluate the efficacy of PTMC film (Flexisurge Adhesion Barrier ${ }^{\circledR}$, Medisse BV, Ede, The Netherlands) as an anti-adhesive material, we compared this material to two commercially available anti-adhesive therapies (Seprafilm ${ }^{\circledR}$ and Adept $^{\circledR}$ ) and a control group in a rat adhesion model. We hypothesize that the PTMC film provides adequate adhesion prevention, at least comparable to that of commercially available therapies. 
Since abdominal surgery is often performed to resect parts of the bowel, it should be investigated if the use of the PTMC film is safe in the presence of bowel anastomoses. The concept that an equilibrium exists between collagen synthesis and lysis, which can result in either adhesion formation or anastomotic leakage is widely accepted ${ }^{19}$. Furthermore, the presence of a foreign body, persistent infection or trauma can cause adhesions ${ }^{20}$. Therefore, it is crucial to examine if the PTMC film will still be effective in an infected milieu, without aggravating the infection. In this study, we additionally investigated safety of use of the PTMC film both in the presence of a colonic anastomosis and in the case of polymicrobial sepsis in an experimental rat model.

\section{Materials and methods}

\section{Materials}

Poly(1,3-trimethylene carbonate) (PTMC) was polymerized at $150^{\circ} \mathrm{C}$ by ring opening polymerization of 1,3-trimethylene carbonate (For You Medical, P.R. China). Ring opening polymerization was performed under a nitrogen blanket using stannous octoate (Sigma Aldrich, U.S.A.) as a catalyst. Subsequently, the PTMC polymer was compression molded into films, without additional purification. This was done at $160^{\circ} \mathrm{C}$ using a LabEcon 600 press (Fontijne Grotness, The Netherlands). Films produced had an average thickness of $150 \mu \mathrm{m}$. Sterilization with simultaneous cross-linking was performed under inert atmosphere using gamma-radiation from a 60Co source with a dose of 25kGy. An A-B-A PTMC-PEGPTMC tri-block co-polymer was synthesized at $140^{\circ} \mathrm{C}$ by ring opening polymerization of 1,3-trimethylene carbonate using poly(ethylene glycol) (PEG) (Sigma Aldrich, U.S.A) as initiator.

Commercially available products of Hyaluronate Carboxymethylcellulose (Seprafilm ${ }^{\circledR}$, Genzyme Biosurgery, Sanofi, The Netherlands) and Icodextrin 4\% (Adept ${ }^{\circledR}$ Adhesion Reduction Solution, Baxter, The Netherlands) were purchased and used according to the manufacturer's instructions. Prior to surgery, Hyaluronate Carboxymethylcellulose sheets were cut to $5 \times 7 \mathrm{~cm}$ patches under sterile conditions. The experimental PTMC sheets were provided as individually packed sterile films measuring 9x6 cm and were cut to a size of $5 \times 7 \mathrm{~cm}$ before operation.

\section{Animals}

Ninety adult male Wistar rats (Harlan, Horst, The Netherlands) with a body weight of 200-250g were housed at the Central Animal Facilities of the Maastricht 
University. Male rats were chosen because of their anatomy that allows scrotal fat to form adhesions quite easily. Furthermore, studies have showed that female hormones can affect adhesion formation ${ }^{21,22}$. Animals were cared for according to local standards and were provided with free access to food and water. The experimental protocol complied with the Dutch Animal Experimental Act and was approved by the Ethical Committee of Animal Experiments.

\section{Study design}

The experiment was divided into three parts. In the first part, the efficacy of the PTMC film was compared to commercially available anti-adhesion products $(n=44$, $\mathrm{n}=11$ /group) with a follow-up of 14 days:

- Group 1 was implanted with the PTMC film. This film was fixed intraperitoneally with the tacky copolymer layer directed towards the visceral organs. To prevent migration of the film, additional fixation of the corners to the abdominal wall using 4 polyglactin 4-0 $\left(\right.$ Vicryl $\left.^{\circledR}\right)$ sutures was applied.

- Group 2 had the HA-CMC film implanted intraperitoneally. Application of the film was done according to manufacturer's instructions.

- Group 3 received an intraperitoneal dose of $5 \mathrm{ml}$ Icodextrin 4\%, which corresponds to $1,51 \mathrm{in}$ an adult male subject of $75 \mathrm{~kg}$.

- Group 4 had no material implanted and was considered the control group.

In the second part, the effect of the PTMC film on a colonic anastomosis was investigated ( $n=26, n=13$ /group), and in the third part a cecal ligation and puncture model was used to compare infection rate between the PTMC group and a control group ( $n=20, n=10 /$ group). Follow up was 7 days in the safety studies.

\section{Surgical procedure efficacy study}

Prior to surgery, all animals received a subcutaneous injection of $0,05 \mathrm{mg} / \mathrm{kg}$ Buprenorphine. Anaesthesia was induced with Isoflurane 5\% and maintained with Isoflurane $2.5 \%$. The abdomen was shaved and disinfected, and the animals were placed in the supine position. A 5-6 cm midline incision was performed, through which the abdomen was accessed. Ischemic buttons were created according to the technique described by Rajab et al. ${ }^{23}$. In short, musculoperitoneal tissue was lifted using surgical forceps after which a suture was run through the base of the button. A ligature was made on one side of the button, followed by a similar ligature around the complete base of the button. Using this technique, six buttons were created in each animal, three on each side of the midline. The buttons had a diameter of approximately $0.5 \mathrm{~cm}$, and were spaced $1 \mathrm{~cm}$ apart (Figure 9.1)23,24. 
Subsequently, a $1 \times 1 \mathrm{~cm}$ portion of the tip of the cecum was abraded using a sterile cotton swab until petechial lesions occurred ${ }^{25,26}$. After euthanasia with an inhalation overdose of carbon dioxide, the abdomen was opened through an $\mathrm{H}$ shaped incision along the old midline incision, and towards the flanks caudal and cranial of the ischemic buttons. Care was taken to avoid dissection through the ischemic buttons or through existing adhesions. The amount of adhesions to the ischemic buttons, midline, cecum, or sutures was scored macroscopically.

\section{Surgical procedure safety study}

A rat model for colonic anastomosis was used in which the abdominal cavity was accessed through a $5 \mathrm{~cm}$ craniocaudal midline incision of the skin and abdominal musculature. The cecum was identified and placed on sterile gauzes hydrated with sterile saline solution to prevent dehydration. The colon was transected $2 \mathrm{~cm}$ distal from the cecum and an end-to-end anastomosis was created using 12 interrupted polypropylene 6/0 sutures (Prolene, Ethicon, Johnson \& Johnson) (Figure 9.2a), after which the intestines were repositioned into the abdominal cavity.

To induce a controlled infection cecal ligation and puncture was performed as previously described 27,28 . In this model, the cecum was carefully manipulated outside the abdominal cavity and ligated just distal to the ileocecal valve with a monofilament non-absorbable suture (4/0 Ethilon ${ }^{\circledR}$; Ethicon, Johnson \& Johnson, Somerville, New Jersey, USA, Figure 9.2b), maintaining the continuity of the bowel. The caecum was punctured distally to the ligation with an $18-\mathrm{G}$ needle.

Both intervention groups received the PTMC film, which was fixed intraperitoneally covering the visceral organs. For means of hydration a bolus of $2 \mathrm{ml}$ sterile saline solution was injected s.c.. In all experiments, the abdominal wall was closed using an absorbable running suture of polyglactin 4-0 (Vicryl ${ }^{\circledR}$; Ethicon, Johnson \& Johnson). The skin was closed intracutaneously with a running suture of polyglecaprone 4-0 (Monocryl ${ }^{\circledR}$; Ethicon, Johnson \& Johnson).

\section{Adhesion scoring}

Adhesions to buttons were scored by two independent observers. The amount of buttons with adhesions present was recorded. Data were presented as mean number of buttons with adhesions. Adhesions to the abraded cecum and midline were recorded in a similar fashion. 


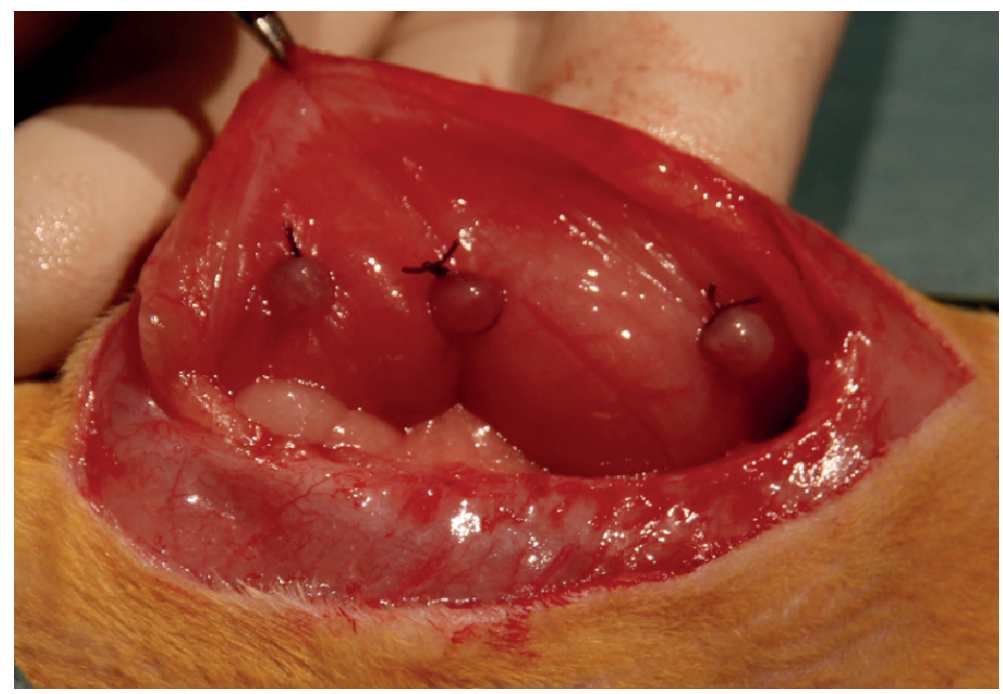

Figure 9.1 Three ischemic buttons were created on each side of the abdominal wall.

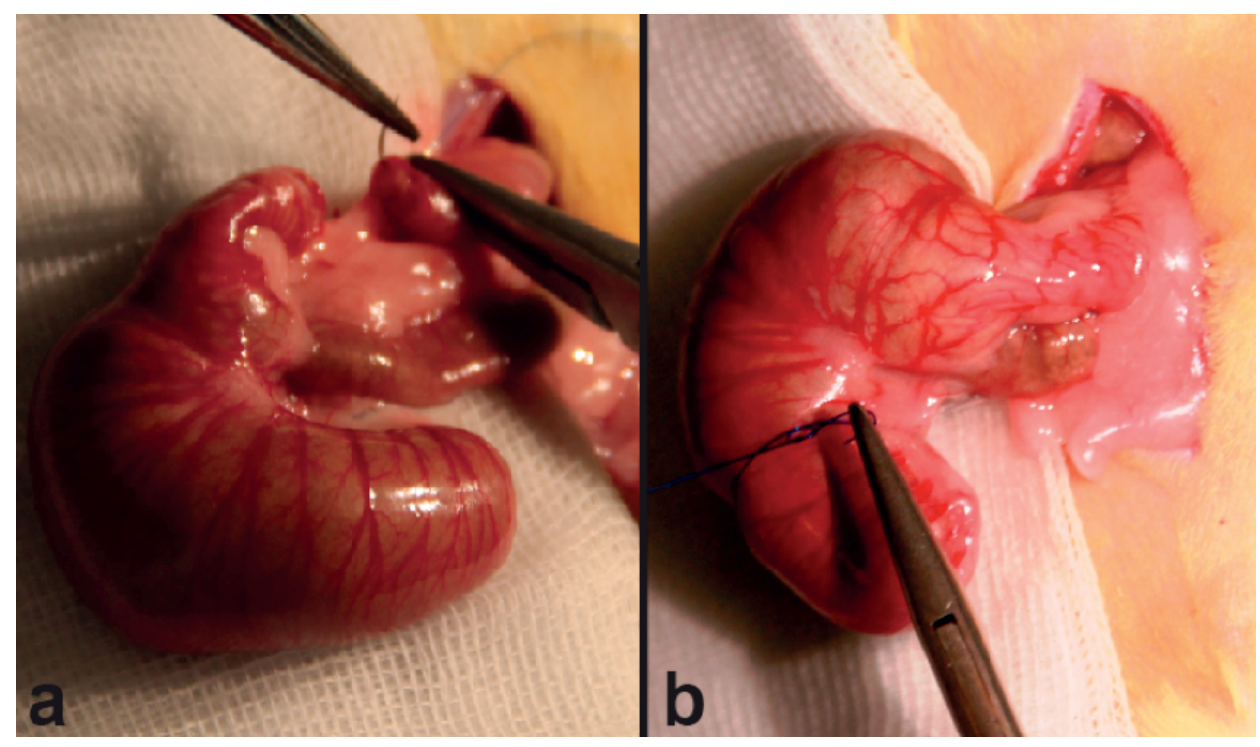

Figure 9.2 Safety of PTMC in the abdominal cavity was investigated using an anastomotic leakage model (a) and a cecal ligation and puncture model (b). 


\section{Examination of anastomotic leakage}

Anastomotic leakage was defined as $0=$ no anastomotic leakage, $1=$ small abscess at the anastomotic site $<1 \mathrm{~cm}^{3}, 2=\operatorname{large}\left(>1 \mathrm{~cm}^{3}\right)$ abscess at the anastomotic site, $3=$ faecal pollution of the abdomen, $4=$ complete dehiscence with peritonitis. Adhesions at the anastomotic site were evaluated in a blinded fashion according to the scoring scale of van der Ham ${ }^{29}$. After sacrifice, the anastomotic segment was resected and paraffin-embedded sections were prepared. Sections were stained with haematoxylin and eosin using standard histological techniques. Specimens were randomly scored according to the 0-4 Ehrlich and Hunt numerical scale as modified by Phillips et al. ${ }^{30}$.

\section{Bursting pressure}

A 5-cm segment of intestine including the anastomosis with and adherent organs was resected en bloc. The colon distally of the anastomosis was clamped, a plastic tube was inserted in the proximal end and ligated with a single polyglactine $4 / 0$ suture (Vicryl, Ethicon). Each anastomosis was immersed in 1x phosphate buffered saline, air was infused using a manometer (IDEE, Maastricht University, the Netherlands) and pressure was manually increased by inflating the colon with air. The bursting pressure of the anastomosis was defined as the intraluminal pressure at which air leakage was initially observed from the anastomosis (mBar).

\section{Infection rate}

The day after the cecal ligation and puncture, the abdomen was re-opened through the midline incision, and a culture swab of the abdominal cavity was obtained to confirm fecal peritonitis. At sacrifice, this action was repeated to measure infection at day 7. Swabs were analyzed using broad-range $16 \mathrm{~S}$ ribosomal RNA (rRNA) gene polymerase chain reaction (PCR) for detection and identification of bacterial pathogens. Furthermore, plasma was collected with an intracardial puncture to perform a blood culture to check for sepsis.

\section{Statistical analysis}

All data concerning adhesions are expressed as median with range. Normality tests using the Kolmogoroc-Smirnov test were performed. Non-parametric data was analysed using the Kruskal-Wallis test. For categorical data a Fisher's exact test was performed. In case of significance, the difference was confirmed by the MannWhitney test, followed by a Bonferroni post-hoc test. A $P$-value $<0.008$ was considered statistically significant. Statistical analyses were performed using Prism 5.0 for Mac (Graphad software, Inc, San Diego, CA) and SPSS 20.0 for Mac (SPSS Inc, Chicago, IL). 


\section{Results}

Two animals died during follow-up in the efficacy study. One animal from group 3 died preoperatively, possibly due to an overdose of anaesthetic. Another animal from group 1 died five days after the initial operation; this was due to a severe sepsis caused by leakage of bowel content through a cecal perforation. All other animals showed a normal postoperative recovery. In the safety studies, all animals completed the 7-day follow-up. Welfare of animals in both the efficacy and safety studies was assessed using extensive scoring, but no humane end points were reached and no significant differences were found between intervention and control groups.

\section{Macroscopic evaluation}

The PTMC films showed no significant signs of degradation after 14 days and could be explanted at sacrifice. The film was still in situ in all of the surviving animals. Contrary to PTMC, the Icodextrin and HA-CMC were completely resorbed after 14 days. None of the surviving animals in the efficacy study showed macroscopic signs of infection or severe inflammatory reaction. In the safety studies, signs of inflammation and discomfort were found, but were equal in both PTMC and control groups.

\section{The use of a PTMC film reduces adhesion formation}

PTMC films had the lowest amount of adhesions to ischemic buttons of all studied groups with a median of 0.5 (range: $0-3$ ) buttons per animal. This difference was statistically significant to both the control group (median: 4 range: 2-6) and Icodextrin group (median: 4.5 , range: $0-6)(P<0,001)$ but not to the HA-CMC implant group (median 2, range: $0-5, P=0.04$ ). Results of adhesion formation to the ischemic buttons are presented in Figure 9.3 and Figure 9.4

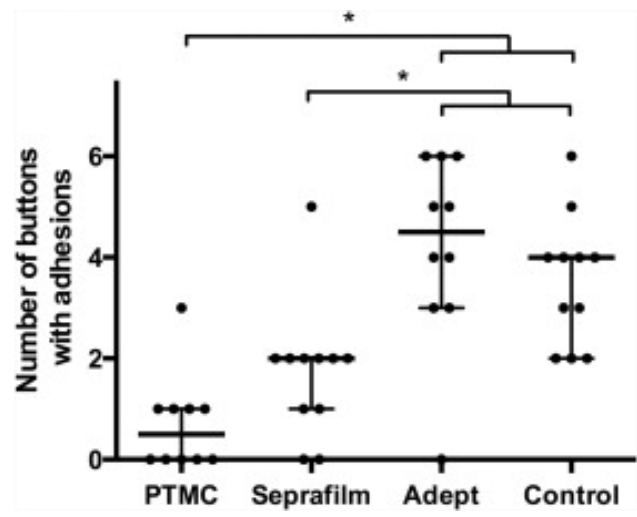

Figure 9.3

Number of buttons with adhesions per group. Data are presented with median \pm interquartile range. ${ }^{*} P<0.008$ 

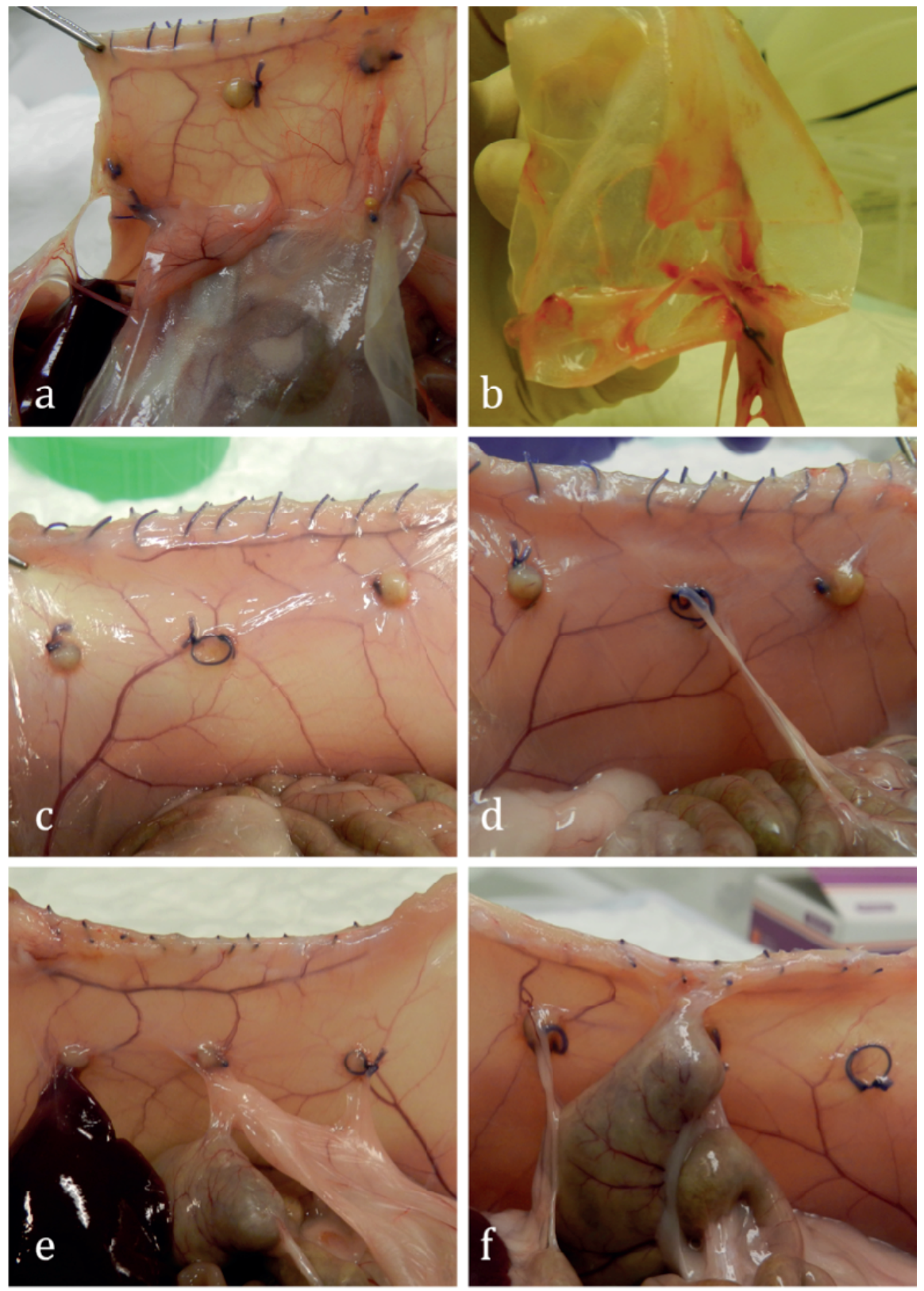

Figure 9.4 PTMC film remains intact and in situ after 14 days retaining its mechanical characteristics. Sutures fixing the film are prone to adhesion formation $(\mathrm{a}+\mathrm{b})$. Pictures $\mathrm{c}$ and $\mathrm{d}$ show absent to minor adhesion formation, with involvement of omental or scrotal fat only as seen in most PTMC and HA-CMC animals. Pictures e and f show severe adhesions with involvement of visceral organs as seen in some of the animals in the control group or in the Icodextrin group. 
Adhesions in the PTMC and HA-CMC groups appeared to be mostly filmy and of omental and scrotal fat origin. None of these adhesions required aggressive or sharp dissection. No adhesions of visceral organs to the buttons were found in these groups. In contrast, the control and Icodextrin groups showed more pronounced dense adhesions. These adhesions required more aggressive blunt and sharp dissection. Adhesions of liver, cecum and small intestine to the ischemic buttons were recorded besides the usual fatty adhesions in these groups. The use of sutures to fix the PTMC to the abdominal wall, however, seems to increase the risk of undesirable adhesions to these sutures. Although the film seems to protect the ischemic buttons from adhesions forming to these buttons, the sutures are not protected. All of the eleven animals in the PTMC group had adhesions present attached to sutures fixing the film. These adhesions were denser and more difficult to dissect than those to the ischemic buttons in the same animals. Furthermore, most adhesions to ischemic buttons in this group seemed to be closely related to adhesions formed to these sutures. No differences were found in the amount of adhesions adhered to the abraded cecum. In all but the PTMC group, 4 out of the total 11 animals have some sort of adhesion towards the abraded cecum. In the PTMC group only 2 animals showed adhesions towards the cecum. No significant results were found between the groups using the Fisher's exact test (Figure 9.5A).

\section{Equal anastomotic leakage in both PTMC and control group}

The anastomoses in the PTMC group needed higher pressure to burst $(249 \pm 14.2$ mBar versus $195 \pm 22.0 \mathrm{mBar}$ ), however this was not significant $(P=0.067$, Figure 9.5B). We found no higher anastomotic leakage rate in the PTMC group compared to the control group $(P=1.00$, Figure $9.5 \mathrm{C})$ and also the severity of anastomotic leakage did not differ between groups $(P=0.695$, Figure 9.5D). Microscopically, no differences were found between experimental groups.

\section{No differences in infection rate between experimental groups}

Bacterial load at day 7 did not differ between PTMC (3.1*108 copies/ $\mu$ l) and control groups $(8.1 * 108$ copies $/ \mu \mathrm{l}, P=0.35$, Figure $9.5 \mathrm{E})$. There were no differences in the frequency of positive versus negative blood cultures between groups $(P=0.637$, Figure 9.5F). 
A

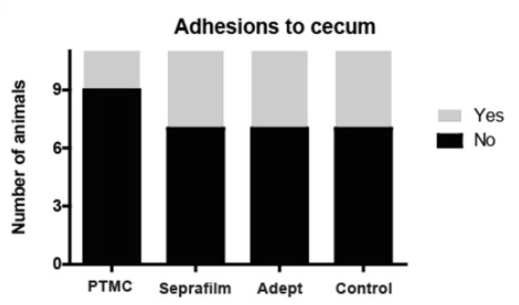

B

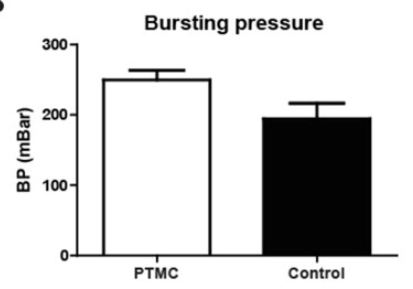

C

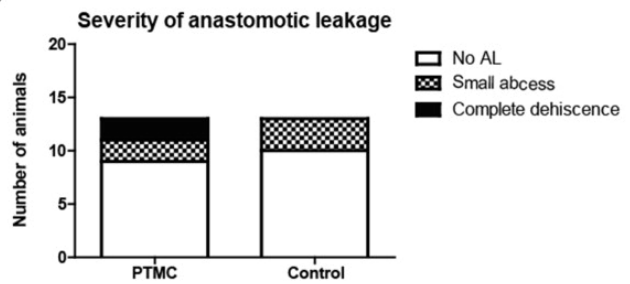

D

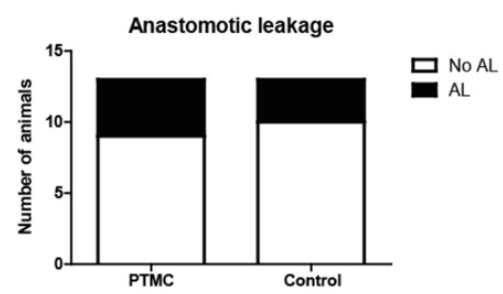

E

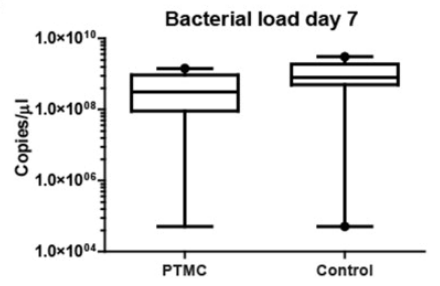

$\mathbf{F}$

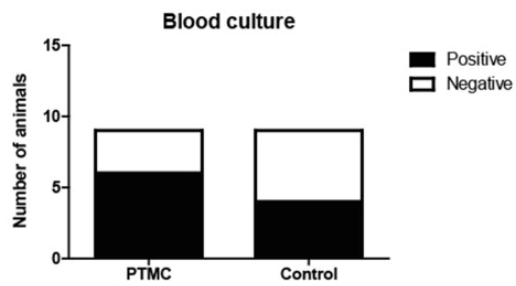

Figure 9.5 Results of safety study. No differences were detected between groups in percentage of animals per group with adhesions present from the abraded cecum to other structures within the peritoneal cavity (Figure A). Bursting pressure was slightly greater in the PTMC group $(249 \pm 14 \mathrm{mBar})$ compared with the control group $(195 \pm 22 \mathrm{mBar} ; P=0.067$, Figure B), There was no difference in anastomotic leakage rate between the poly(trimethylene carbonate) (PTMC) and control group (Figure $\mathrm{C}+\mathrm{D}$ ). When the severity of anastomotic leakage was studied into more detail, a complete dehiscence of the anastomosis could only be found in the PTMC group $(P=0.695)$. Bacterial load at day 7 did not differ between PTMC $(3.1 * 108$ copies/ $\mu \mathrm{l}$; interquartile range [IQR], 0.58) and control groups $\left(8.1^{*} 108\right.$ copies/ $\mu \mathrm{l} ; \mathrm{IQR}, 2.49 ; P=0.35$, Figure E). No differences were found in the frequency of positive versus negative blood cultures between groups $(P=0.637$, Figure $\mathrm{E})$.

\section{Discussion}

In this study we investigated a new anti-adhesive barrier composed of PTMC (co)polymers and compared it to commercially available anti-adhesive therapies. Prevention of intraperitoneal adhesions remains an integral part of daily surgical practice $^{6}$. Postoperative adhesions are known to have a devastating impact on 
quality of life and increases the risk for reoperations ${ }^{31}$. Even though the use of minimally invasive techniques seems to reduce the risk of adhesion formation, this is not sufficient to adequately prevent all postoperative adhesions, indicating there is still need for additional adhesion prevention ${ }^{1,15,32,33}$. PTMC is a highly biocompatible and versatile material with highly favourable characteristics ${ }^{34,35}$. Contrary to currently available materials, the PTMC film degrades by surface degradation, not by bulk degradation. This allows the material to retain its mechanical characteristics and give prolonged separation of adhering tissues ${ }^{34-36}$. Furthermore, degradation of PTMC induces only mild inflammatory reaction leading to the hypothesis that intraperitoneal placement of PTMC film gives a reduction of postoperative adhesions comparable to or even better than commercially available materials ${ }^{34}$.

The results of this experiment indicate PTMC film (FlexiSurge Adhesion Barrier ${ }^{\circledR}$ ) does lead to a significant reduction in the amount of adhesions. The fact that the PTMC results are comparable to HA-CMC (Seprafilm ${ }^{\circledR}$ ), which is also a solid film, indicates that physical barriers are an effective way to prevent adhesions. As a more slowly degrading material, PTMC provides long-term and effective separation of tissues since degradation happens through surface erosion. This reduction is significant compared to the control group, indicating the application of a physical barrier is beneficial in adhesion prevention. Surprisingly, the liquid adhesion barrier did not lead to a reduction in the amount of adhesions. Although human trials are inconclusive ${ }^{37}$, Icodextrin $\left(\right.$ Adept $\left.^{\circledR}\right)$ does seem to be effective in several other studies 8,38 .

Furthermore, there are experimental studies indicating that Icodextrin is also effective in rat models, showing less adhesion formation compared to Ringers lactate ${ }^{13,39}$. However, in these studies Icodextrin solution was either provided in higher volumes or in higher concentrations, possibly allowing the liquid to remain effective for longer periods of time. It is assumed that the reason for a reduced effectiveness in rats is the presence of $\alpha$-amylase in the peritoneal fluid of rats, leading to a faster resorption of the Icodextrin fluid, reducing the duration the fluid remains present in the abdominal cavity ${ }^{13,40}$. This could have resulted in the ineffectiveness of the fluid in our study.

Another strong aspect of the new PTMC film is its handling. In contrast to the HA-CMC film, PTMC is highly flexible with sufficient tensile strength, allowing for easy positioning and repositioning within the first few minutes after implantation ${ }^{18,34}$. Whereas the HA-CMC film is reported to be brittle, sticky, and difficult to apply, limiting its use in surgical practice ${ }^{15,41}$. 
Furthermore, the HA-CMC barrier was completely resorbed in all animals after the 14 days follow-up, whereas the PTMC film kept its structural integrity at least until the 14th day. Although peritoneal wound healing is completed after 7 to 10 days, the HA-CMC material loses its structural integrity within the first 24 hours by turning into a hydrophilic gel ${ }^{14,42,43}$. Because the PTMC material degrades through surface erosion it might provide adequate tissue separation for a longer period of time ${ }^{16,34}$.

Since damage to mesothelial lining and the subsequent fibrotic response are considered to be key components in adhesion formation, the main focus of adhesion prevention should lie within the first 7 to 10 days ${ }^{14,42}$. For this reason, we think a follow-up of 14 days provides adequate information on the effect of PTMC barrier on adhesion prevention. Besides, after 14 days, the presence of an intact barrier shows PTMC exceeds the duration of protection of both HA-CMC and Icodextrin. Even though adhesion formation can cause long term detrimental effects on the quality of life of patients, in some cases adhesion formation is necessary, for example in anastomotic healing. It has been shown that certain antiadhesive products may predispose to peritonitis and anastomotic dehiscence ${ }^{11,44}$. In order to address this issue, we placed PTMC film in both the presence of a colonic anastomosis as well as in a model for controlled infection. In both models, the presence of the PTMC film was safe and did not cause any increase in anastomotic leakage rate, nor did it aggravate the induced infection.

Unfortunately, at this moment PTMC film needs suture fixation to keep adequate positioning throughout the follow-up. Although the PTMC reduced the amount of adhesions to the ischemic buttons significantly, unprotected sutures fixing the film seemed to induce adhesion formation at this site. The need for sutured fixations was one of the limitations for practical PTMC film application. Before the film can be tested in a clinical setting, the fixation strength of an attached tacky copolymer layer should be sufficient to provide sutureless fixation.

Another limitation in this study is the possible lack of power to detect any significant differences in amount of adhesions to the cecal abrasion. Although the results of PTMC films are excellent for the adhesions to the ischemic buttons, the effectiveness in preventing adhesions in other locations in the abdomen could not be shown in this model. However, there still seems to be a small, but not significant, reduction in the cecal adhesion formation, which could have clinical implications in larger groups. 


\section{Conclusion}

A new PTMC film with a tacky PTMC-PEG layer is effective in reducing postoperative adhesions to the abdominal wall in a rat ischemic button model. Furthermore, the use of this film does not compromise anastomotic healing nor peritonitis. The proven efficacy, safety and easy handling of the PTMC film make it a promising new prevention tool in postoperative adhesions. Further research is necessary to elucidate whether these results are also valid in humans. 


\section{References}

1. Lower AM, Hawthorn RJS, Clark D, Boyd JH, Finlayson AR, Knight AD, et al. Adhesion-related readmissions following gynaecological laparoscopy or laparotomy in Scotland: an epidemiological study of 24046 patients. Hum Reprod. Oxford University Press; 2004;19:1877-1885.

2. Ellis H, Moran BJ, Thompson JN, Parker MC, Wilson MS, Menzies D, et al. Adhesion-related hospital readmissions after abdominal and pelvic surgery: a retrospective cohort study. The Lancet. 1999; 353: 476-1480.

3. Parker MC, Wilson MS, van Goor H, Moran BJ, Jeekel J, Duron JJ, et al. Adhesions and Colorectal Surgery - Call for Action. Colorectal Disease. Blackwell Publishing Ltd; 2007;9:66-72.

4. Nieuwenhuijzen M, Reijnen MM, Kuijpers JH, van Goor H. Small bowel obstruction after total or subtotal colectomy: a 10-year retrospective review. Br J Surg. Blackwell Science Ltd; 1998;85: 1242-1245.

5. Rajab TK, Wallwiener M, Talukdar S, Kraemer B. Adhesion-related complications are common, but rarely discussed in preoperative consent: a multicenter study. World J Surg. Springer-Verlag; 2009; 33:748-750.

6. Ellis H, Crowe A. Medico-legal consequences of post-operative intra-abdominal adhesions. Int J Surg. 2009;7:187-191.

7. Schreinemacher MHF, Broek ten RP, Bakkum EA, van Goor H, Bouvy ND. Adhesion awareness: a national survey of surgeons. World J Surg. Springer-Verlag; 2010;34:2805-2812.

8. Broek ten RPG, Stommel MWJ, Strik C, van Laarhoven CJHM, Keus F, van Goor H. Benefits and harms of adhesion barriers for abdominal surgery: a systematic review and meta-analysis. The Lancet. 2014;383:48-59.

9. Kumar S, Wong PF, Leaper DJ. Intra-peritoneal prophylactic agents for preventing adhesions and adhesive intestinal obstruction after non-gynaecological abdominal surgery. Cochrane Database Syst 2009.

10. Metwally M, Watson A, Lilford R, Vandekerckhove P. Fluid and pharmacological agents for adhesion prevention after gynaecological surgery. Metwally M, editor. Cochrane Database Syst Rev. Chichester, UK: John Wiley \& Sons, Ltd; 2006;: CD001298.

11. Fazio VW, Cohen Z, Fleshman JW, van Goor H, Bauer JJ, Wolff BG, et al. Reduction in adhesive smallbowel obstruction by Seprafilm adhesion barrier after intestinal resection. Dis Colon Rectum. 2006;49:1-11.

12. Ahmad G, Duffy JMN, Farquhar C, Vail A, Vandekerckhove P, Watson A, et al. Barrier agents for adhesion prevention after gynaecological surgery. Ahmad G, editor. Cochrane Database Syst Rev. Chichester, UK: John Wiley \& Sons, Ltd; 2008;CD000475.

13. Wallwiener M, Brucker S, Hierlemann H, Brochhausen C, Solomayer E, Wallwiener C. Innovative barriers for peritoneal adhesion prevention: liquid or solid? A rat uterine horn model. Fertil Steril. 2006;86:1266-1276.

14. Boland GM, Weigel RJ. Formation and Prevention of Postoperative Abdominal Adhesions. Journal of Surgical Research. 2006;132:3-12.

15. Brochhausen C, Schmitt VH, Planck CNE, Rajab TK, Hollemann D, Tapprich C, et al. Current strategies and future perspectives for intraperitoneal adhesion prevention. J Gastrointest Surg. Springer-Verlag; 2012;16:1256-1274.

16. Pêgo AP, Van Luyn MJA, Brouwer LA, van Wachem PB, Poot AA, Grijpma DW, et al. In vivo behavior of poly(1,3-trimethylene carbonate) and copolymers of 1,3-trimethylene carbonate with D,Llactide or epsilon-caprolactone: Degradation and tissue response. J Biomed Mater Res A. Wiley Subscription Services, Inc., A Wiley Company; 2003;67:1044-1054.

17. Van Leeuwen AC, Huddleston Slater JJR, Gielkens PFM, de Jong JR, Grijpma DW, Bos RRM. Guided bone regeneration in rat mandibular defects using resorbable poly(trimethylene carbonate) barrier membranes. Acta Biomater. 2012;8:1422-1429.

18. Van Leeuwen AC, Van Kooten TG, Grijpma DW, Bos RRM. In vivo behaviour of a biodegradable poly(trimethylene carbonate) barrier membrane: a histological study in rats. J Mater Sci Mater Med. Springer US; 2012;23:1951-1959. 
19. Hendriks DT, Mastboom WJB. Healing of experimental intestinal anastomoses. Dis Colon Rectum. Springer-Verlag; 1990;33:891-901.

20. Lauder CIW, Garcea G, Strickland A, Maddern GJ. Abdominal adhesion prevention: still a sticky subject? Dig Surg. Karger Publishers; 2010;27:347-358.

21. Bozkurt S, Yuzbasioglu MF, Bulbuloglu E, Gul M, Kale IT. Prevention of Postoperative Peritoneal Adhesions by Administration of Estrogen. J Invest Surg. 2009;22:263-267.

22. Kaya U, Oktem M, Zeyneloglu HB, Ozen 0, Kuscu E. Impact of aromatase inhibitors on adhesion formation in a rat model. Fertil Steril. 2007;87:934-939.

23. Rajab TK, Wauschkuhn CA, Smaxwil L, Kraemer B, Wallwiener M, Wallwiener CW. An improved model for the induction of experimental adhesions. J Invest Surg. 2010;23:35-39.

24. Rajab TK, Smaxwil L, Wallwiener M. Animal model for local pharmacotherapy in adhesion prophylaxis--a proof of concept. J Invest Surg. 2013;26:200-203.

25. Chaturvedi AA, Lomme RMLM, Hendriks T, van Goor H. Prevention of postsurgical adhesions using an ultrapure alginate-based gel. Br J Surg. John Wiley \& Sons, Ltd; 2013;100:904-910.

26. Lalountas MA, Ballas KD, Skouras C, Asteriou C, Kontoulis T, Pissas D, et al. Preventing intraperitoneal adhesions with atorvastatin and sodium hyaluronate/carboxymethylcellulose: a comparative study in rats. Am J Surg. 2010;200:118-123.

27. Hubbard WJ, Choudhry M, Schwacha MG, Kerby JD, Rue LW, Bland KI, et al. Cecal ligation and puncture. Shock. 2005;24 Suppl 1:52-57.

28. Deerenberg EB, Mulder IM, Ditzel M, Slieker JC, Bemelman WA, Jeekel J, et al. Polyvinyl alcohol hydrogel decreases formation of adhesions in a rat model of peritonitis. Surg Infect (Larchmt). 2012;13:321-325.

29. van der Ham AC, Kort WJ, Weijma IM, Jeekel H. Transient protection of incomplete colonic anastomoses with fibrin sealant: an experimental study in the rat. J Surg Res. 1993;55:256-260.

30. Phillips JD, Kim CS, Fonkalsrud EW, Zeng H, Dindar H. Effects of chronic corticosteroids and vitamin a on the healing of intestinal anastomoses. The American Journal of Surgery. 1992;163: 71-77.

31. Broek ten RPG, Issa Y, van Santbrink EJP, Bouvy ND, Kruitwagen RFPM, Jeekel J, et al. Burden of adhesions in abdominal and pelvic surgery: systematic review and met-analysis. BMJ. 2013;347: f5588.

32. Schnüriger B, Barmparas G, Branco BC, Lustenberger T, Inaba K, Demetriades D. Prevention of postoperative peritoneal adhesions: a review of the literature. Am J Surg. 2011;201:111-121.

33. Broek ten RPG, Kok-Krant N, Bakkum EA, Bleichrodt RP, van Goor H. Different surgical techniques to reduce post-operative adhesion formation: a systematic review and meta-analysis. Hum Reprod Update. Oxford University Press; 2013;19:12-25.

34. Bat E, van Kooten TG, Harmsen MC, Plantinga JA, van Luyn MJA, Feijen J, et al. Physical properties and erosion behavior of poly(trimethylene carbonate-co-e-caprolactone) networks. Macromol Biosci. WILEY-VCH Verlag; 2013;13:573-583.

35. Zhang Z, Kuijer R, Bulstra SK, Grijpma DW, Feijen J. The in vivo and in vitro degradation behavior of poly (trimethylene carbonate). Biomaterials. 2006.

36. Bat E, Van Kooten TG, Feijen J, Grijpma DW. Macrophage-mediated erosion of gamma irradiated poly (trimethylene carbonate) films. Biomaterials. 2009.

37. Broek MD ten RPG, MD MWJS, MD CS, van Laarhoven MD PCJHM, MD FK, van Goor FRCS PH. ArticlesBenefits and harms of adhesion barriers for abdominal surgery: a systematic review and meta-analysis. The Lancet. Elsevier Ltd; 2013:1-12.

38. Tepetes K, Asprodini EK, Christodoulidis G, Spyridakis M, Kouvaras E, Hatzitheofilou K. Prevention of postoperative adhesion formation by individual and combined administration of 4 per cent icodextrin and dimetindene maleate. Br J Surg. John Wiley \& Sons, Ltd; 2009;96:1476-1483.

39. Klink CD, Schickhaus P, Binnebösel M, Jockenhoevel S, Rosch R, Tolba R, et al. Influence of 4\% icodextrin solution on peritoneal tissue response and adhesion formation. BMC Surg. BioMed Central Ltd; 2013;13:34.

40. van den Tol P, Raa ten S, van Grevenstein H, Marquet R, van Eijck C, Jeekel H. Icodextrin reduces postoperative adhesion formation in rats without affecting peritoneal metastasis. Surgery. 2005; 137:348-354. 
41. Ward BC, Panitch A. Abdominal adhesions: current and novel therapies. Journal of Surgical Research. 2011.

42. Hellebrekers BWJ, Kooistra T. Pathogenesis of postoperative adhesion formation. Br J Surg. John Wiley \& Sons, Ltd; 2011;98:1503-1516.

43. Brochhausen C, Schmitt VH, Rajab TK, Planck CNE, Kraemer B, Wallwiener M, et al. Intraperitoneal adhesions--an ongoing challenge between biomedical engineering and the life sciences. J Biomed Mater Res A. Wiley Subscription Services, Inc., A Wiley Company; 2011;98:143-156.

44. Tang C-L, Jayne DG, Seow-Choen F, Ng Y-Y, Eu K-W, Mustapha N. A Randomized Controlled Trial of $0.5 \%$ Ferric Hyaluronate Gel (Intergel) in the Prevention of Adhesions Following Abdominal Surgery. Annals of Surgery. 2006;243:449-455. 


\section{CHAPTER 10}

General discussion 
Chapter 10 


\section{General discussion}

Over 15,500 patients are diagnosed with colorectal cancer (CRC) in the Netherlands every year, making it the most common oncological disease in $2015^{1}$. Due to national screening programs and a growing elderly patient population the incidence of CRC is expected to rise in the coming years, increasing the frequency of colorectal cancer treatable by surgery ${ }^{2}$. Two thirds of patients with CRC will undergo surgical treatment involving resection of the tumour, since surgery remains the cornerstone of treatment. Therefore, the perioperative course of colorectal surgery is of great importance and relates strongly with clinical outcome in terms of mortality and functional recovery. In recent decades, perioperative care has significantly improved due to advances in anaesthesia, minimally invasive surgical techniques and the introduction of 'fast track' protocols ${ }^{3}$. However, complications after colorectal surgery still occur regularly, of which anastomotic leakage (AL) is the most feared. AL develops when the construction of two intestinal ends - the anastomosis - does not heal properly; subsequently, intestinal content leaks into the abdominal cavity causing abscess formation and peritonitis.

In 2014, 712 of the 10.426 (6.8\%) nationwide registered cancer patients who underwent colorectal resection developed severe AL (447/7580 [5.9\%] colon and 265/2846 [9.3\%] rectum) that required re-intervention (Dutch Surgical Colorectal Audit, 2014). These patients suffered from high morbidity, prolonged hospitalization and even mortality. Moreover, some studies even suggested that AL is associated with reduced disease-specific survival and increased recurrence rates of CRC after colorectal surgery ${ }^{4-6}$.

Despite extensive observational and experimental research in both animal models and in humans, the incidence of AL has remained unchanged over the past decades. There is still no solution for this important clinical problem, despite the increasing amount of studies that have investigated numerous interventions to promote anastomotic healing. We suggest that this is largely because causal factors leading to colorectal AL are still not recognized. And although some patient factors and specific drug treatments have been associated with an increased risk of AL, there is still a large knowledge gap in normal anastomotic healing (Chapter 1).

The process of wound healing in the intestinal tract, upon the construction of an anastomosis, is often compared with the process of wound healing observed in the skin (i.e. cutaneous healing). Intestinal healing indeed takes place in the three wellknown phases of inflammation, proliferation and remodelling7 . However, there are substantial differences, as intestinal healing is 1) faster than cutaneous healing 2) other types of cells and substances are involved, and 3) both the skin and the intestines house unique microbiota that cannot be compared directly ${ }^{8}$. It is essential to distinguish between cell types and functions/reactions of different cells 
to come to understanding of $\mathrm{AL}$, not to mention to find a solution for the clinical burden of AL. Interaction between intraluminal content and the layers of the bowel wall, with their separate cell types and function, may be key in unravelling the healing process. Thus far, certain cell types are known to play a role in anastomotic healing such as matrix metalloproteinases (MMPs)9,10, inflammatory cells as macrophages ${ }^{11}$ and intestinal microbiota ${ }^{12,13}$ but the interaction between these different types and their exact role in time during the healing process is not yet clarified. Therefore, research should refocus on the healing process of colorectal anastomoses and the pathophysiology that results in AL.

\section{Animal models for research on intestinal anastomotic healing}

Laboratory animals are often used to study intestinal healing and ways to prevent anastomotic leakage; several models exist in which an anastomosis is being constructed. However, none of these models are completely translatable to the human setting and it is difficult to compare models between different species. There is still debate on which models are suitable for research on anastomotic healing and leakage in the lower gastrointestinal tract.

To generate more clarity on animal models for AL and to obtain comparability between different studies, it is crucial that researchers agree on what outcome parameters and experimental setup is important in the investigation of intestinal anastomoses. For this reason, we conducted a Delphi analysis (Chapter 2). This is a research method that relies on a panel of experts. These experts are requested to answer a single questionnaire in multiple rounds. After each round, they receive an anonymous summary of the experts' forecasts from the previous round as well as the reasons they provided for their judgments. In this way, experts are encouraged to revise their earlier answers in light of the replies of other members of their panel. It is believed that during this process the range of the answers will decrease and the group will converge towards the "correct" answer ${ }^{14}$. We reasoned that such an approach could lead to consensus among experts investigating AL in animal models regarding anastomoses in the lower gastrointestinal tract, specifically on which animal, location, and type of surgery; macroscopic outcome; histological assessment; mechanical and biochemical outcome measures; and animal testing and welfare.

One of the main outcomes of the Delphi analysis is that dog and rabbit animal models should no longer be considered, and thus used, as validated models for research into anastomotic healing of the lower gastrointestinal tract. The mouse, rat and pig models are considered appropriate, but experiments ought to be executed according to current legislation and reported more into detail. The macroscopic result of anastomotic leakage should always be one of the main outcome parameters, together with the different grades of leakage (small/large 
abscesses, faecal peritonitis, complete dehiscence). None of the available scoring systems in literature regarding AL were considered to be appropriate, however a new 'anastomotic complication score' was proposed by one of the panel members and was directly considered as an appropriate and useful tool by the other experts, although the usability has yet to be proven in practice (i.e. external validation is required). This score also implicates adhesions to the anastomotic site, which are considered relevant to mention as they might cover signs of leakage. Histological assessment of the anastomotic site such as amount of inflammation, vascularisation and collagen deposition is very valuable and considered as an appropriate outcome measure, especially in studies that focus on anastomotic healing. Both bursting pressure and tensile strength are considered appropriate measurements for anastomotic strength, where it should be mentioned that these measurements could be compared within one experiment but due to heterogeneity not between different experiments. There were no additional outcome measures (e.g. amount of collagen, specific immunohistochemical stainings, ELISA measurements or qPCR regarding specific genes) considered appropriate as a surrogate marker for anastomotic healing, but they can provide information on a specific research question.

All animal studies should be reported according to the Animal Research: Reporting of In Vivo Experiments (ARRIVE) guidelines ${ }^{15}$. This improves transparency and increases the quality of animal studies. In addition, blinding and randomization procedures should be reported in animal studies as in human studies. A publicly available online registry (comparable to clinicaltrials.gov for human studies) together with standardized protocols per animal model can aid in advancing the field of animal research on bowel anastomoses. Also, innovative methods such as intestinal organoids or the use of human tissue that can replace animal models should be further investigated to reduce the use of animals, according to the 3R (Replacement, Reduction and Refinement) principle. This was incorporated in the recently published report by the National Advisory Committee on Animal Experiments (NCad), which aims that in 2025 the Netherlands will be the world leader in animal-free innovative research.

Based on previous (animal) experiments, we hypothesize that AL has a complex multifactorial pathophysiology, where at least ischemia, bacteria and inflammation are involved ${ }^{12}$. To study anastomotic healing and leakage in depth, a technique or approach is required that can 1) provide an overview of the healing process at different time points, 2) differentiate between inflammatory markers and various cell types and 3) visualize the site of the leakage in the anastomotic line. Such a technique could help unravel the biological process of anastomotic healing. In this thesis, a start with such a relatively new technique has been made. We are currently studying the anastomotic healing process by means of mass spectrometry imaging 
(Chapter 3). This technique will enable us to identify specific substances in the various layers of the intestinal wall as potential leverage to further unravel the anastomotic healing process. Identifying how the intestinal healing works and where exactly the different phases of healing take place may lead to possible new interventions that can improve healing and eventually cause a lower percentage of AL in the future. Obviously, more research needs to be performed and as any other relatively new technique, it has to be compared to conservative, validated methods to prove its reliability, but it seems to have a high potential and may be the answer to the call for a different approach in the research field of colorectal AL.

\section{The use of knockout mice to identify risk factors for colonic anastomotic leakage}

In the past decades, important risk factors for AL such as male gender ${ }^{16}$, neoadjuvant chemotherapy ${ }^{17}$, tumor size $^{18}$, malnutrition ${ }^{19}$, smoking ${ }^{20}$, steroid treatment ${ }^{21}$ and the use of non-steroidal anti-inflammatory drugs (NSAIDs) ${ }^{22}$ have been identified.

In this thesis, the association between NSAIDs and a higher AL rate is partly elucidated. In chapter 4 it has been demonstrated that depletion or inhibition of Cyclo-oxygenase 2 (COX-2) leads to an increase in the percentage of AL rate in a mouse model, possibly by affecting the blood flow of the intestinal anastomosis, since neoangiogenesis was diminished in these animals, as investigated through CD31 immunohistochemistry. Additionally, COX-2 plays a major role in the inflammatory phase of the healing process and is therefore considered to be essential in the healing process after anastomotic surgery. Moreover, in this same study, we translated our findings from the animal study to the clinical setting by identifying that patients with a specific COX2 polymorphism were more prone to develop colorectal anastomotic leakage.

Among the numerous factors contributing to healing of surgical anastomoses, the intestinal mucus system has been largely overlooked. This is despite the fact that various studies have shown that mucus is essential for protection of the epithelium from luminal challenge ${ }^{23,24}$. Since mucus is a first line of defence against external luminal factors in the gastrointestinal tract, we investigated if depletion of the structural mucus protein Muc2, which is the most abundant mucin in the colon, is associated with impaired colon anastomotic healing (Chapter 5).

We noticed that in Muc2 knockout mice, which lack the main component of mucus in the colon, the rate of AL was significantly higher than that of control animals. Also, when a clear relation between mucus and AL rate in humans is established, one could reason that patients who have an impaired mucous lining in the gut may become eligible to undergo interventions that thicken the mucus. We 
have tried to enhance the mucus layer with prostaglandine E2 $\left(\mathrm{PGE}_{2}\right)$, since it is known that this can stimulate mucus secretion in both small intestine and proximal colon ex vivo. Furthermore, Non-steroidal anti-inflammatory drugs (NSAIDS) inhibit COX enzymes, which leads to a decrease in prostaglandin synthesis. Since there is an association between the use of NSAIDs and AL there must be an interaction between prostaglandins and anastomotic healing. Unfortunately, we did not find a positive influence of $\mathrm{PGE}_{2}$ on anastomotic healing. However, other factors might influence the mucus layer stronger than prostaglandins. For example, experimental studies suggest a positive effect of antibiotics on the strength of colonic anastomosis ${ }^{25,26}$. It is widely accepted that the mucus layer in the colon is the natural habitat for a variety of bacteria and that these bacteria are essential in order to maintain the natural balance in the intestine. One of the tasks of the bacteria in the gut is to break down dietary fibers into smaller particles that we can extract energy from. As a consequence short-chain fatty acids (SCFAs) are being released. These are elements that can be used again by the intestinal cells as a nutrient. If these SCFA-producing bacteria are inhibited, this may lead to a diminished function of enterocytes due to a lack of energy ${ }^{27}$. The balance between pathogenic and commensal bacteria has been described in the frame of different pathologies ${ }^{28-30}$. Recently, the group of professor Alverdy postulated the hypothesis that bacteria play a significant role in the pathophysiology of $\mathrm{AL}^{31}$. Over the past years, they have shown that virulent bacteria with high collagenase activity may contribute to developing $\mathrm{AL}^{12}$. These results strengthen the statement of the previous chapter that in depth research into the pathophysiology of AL may lead to new insights and potential targets that can improve anastomotic healing after colorectal surgery.

\section{Reducing colorectal anastomotic leakage rate: glues and enema interventions}

Many attempts have been made to prevent AL after colorectal surgery, either by enhancing the healing process through different pharmaceutical approaches, mechanically strengthen the anastomosis or by preventing the sequelae of leakage with specific intraluminal devices. All these interventions were tested in animal studies or in the experimental trial setting in humans ${ }^{32-34}$. None of these measures have been incorporated in standard medical care, some because they had negative side effects, others were never tested in humans, but in the most cases it remains unclear why successful attempts have not been investigated further. Recent advances that seem to be promising are to strengthen the anastomotic line, either directly by the applications of sealants or glues around the anastomosis or by influencing the healing process through an increased interaction of colonocytes with their surroundings, such as intraluminal content. 
One of the most abundant short chain fatty acids in the intestine is butyrate, which is also produced by bacteria in the process of digestion ${ }^{35}$. This SCFA has often been investigated in the intestine and it has been shown that it enhances anastomotic strength when it is given as an enema in rats ${ }^{36}$. During the research performed for this thesis, an attempt has been made to come up with a better method than enemas (Chapter 6). This is of interest, since surgeons are often reluctant to give enemas when an intestinal anastomosis is constructed. Clinicians are concerned that the enema by direct mechanical forces or through increased tension on the seam just causes a dehiscence of the anastomosis, in fact causing mechanical AL. The possibility to strengthen proximal anastomosis with topical application prior to construction of the anastomosis was investigated, as well as enrichment of the butyrate enemas with other substances such as hyaluronan since it is known that hyaluronan contributes significantly to cell proliferation and migration ${ }^{37}$. Unfortunately, these experiments yielded few new insights and it seems that butyrate supplementation via an enema remains the best option.

Numerous attempts have been made to reinforce the colonic anastomosis during surgery in order to prevent AL. Commonly used materials are tissue adhesives (TAs), adhesive strips or patches consisting of biological materials $33,38,39$. It is known that certain adhesives such as fibrin glue do not lead to a lower anastomotic leakage rate 40 and that the application of cyanoacrylate in gastrointestinal anastomoses seems promising ${ }^{41}$. This is why our colleagues at the Erasmus Medical Centre started a stepwise approach to detect which TA is the most promising to translate from the experimental setting into the clinical situation. First, they investigated ex vivo that cyanoacrylate is indeed capable of increasing the mechanical strength of anastomoses, both in a normal and a technical insufficient situation ${ }^{42}$ and compared several types of TAs in a rat study ${ }^{43}$. In this latter study it was shown that cyanoacrylates are indeed the most promising group of TAs for colonic sealing, however certain types of cyanoacrylate may have a negative influence on anastomotic healing ${ }^{44,45}$. In Chapter 7 , we examined several cyanoacrylate based commercially available glues and their effect on the occurrence of AL, stenosis and general well being of the animals in a model of a mechanically insufficient colonic anastomosis. Of the three TAs Histoacryl Flex, Glubran 2 and Omnex, the first showed the lowest leakage rate compared to the other TAs whereas Glubran 2 showed the highest AL rate and caused an increased inflammatory response. Despite positive findings in literature, it has also been reported that Glubran 2 causes severe inflammation, with multiple microabscedation in a model for experimental hernia repair ${ }^{46}$. Thus far, it remains unclear why Glubran 2 has a pro-inflammatory effect, especially since there are also reports on the beneficial effects of this adhesive for intestinal healing 43,47 . Histoacryl Flex was associated with the presence of more mature collagen, suggesting to promote anastomotic healing without any harmful reaction. 
Additional in vivo testing has to be performed, especially in a large animal model before this tissue adhesive can be implemented in clinical practice, which is something that is currently being investigated in Rotterdam. The big advantage of Histoacryl Flex seems to be that it has an enhanced flexibility compared to other adhesives and that they appear to adapt easier three-dimensionally. This is of great importance in the mobile intestine where it is known that TAs can cause inflammation and mechanical ileus, which was also the case in our study (5/11 rats in Glubran 2 group).

\section{Potential disruption of the balance between intestinal anastomotic healing and adhesion formation}

Tissue adhesives are studied as preventive measures for AL, since their main functions are to repair injured tissues, reinforce surgical wound or even replace common suturing techniques. Biopolymers can also reach these aims ${ }^{48}$. These polymeric materials can be constructed into three-dimensional networks that can physically of chemically bind to the target tissue and act as hemostats, adhesives or sealants. In this perspective, we also examined the option of a sealing patch able to reduce $\mathrm{AL}$, which was developed in the framework of the European project 'AnastomoSEAL' where we, as a research group, were responsible for the animal experiments. In this project, different consortium members developed a patch with bioactive components from scratch. A patch is a three-dimensional network consisting of several (bio)polymers and the combination of these polymeric agents has a big influence on adhesion mechanisms, mechanical performance and resistance to body fluid. Therefore, these various aspects should be taken into account to choose the most suitable formulation for the target application. The most difficult aspect in the specific development of materials for intra-abdominal use is the degree of adhesion. The aimed material needs to be sticky enough to adhere in a moist environment, but should not be too adhesive since that may cause stenosis or adhesions between the intestines and the abdominal wall. In addition, it is a risk to insert a foreign body material in the vicinity of a complex biological process such as anastomotic healing of which it is impossible to predict what the reaction to the material will be. Substances that may appear promising in vitro may react differently in vivo. Therefore, during the development process of biomaterials not only the main components of the end product should be individually tested, but also the auxiliary substances, since they may lead to unwanted side effects. During the 'AnastomoSEAL' project, we noticed severe adverse events in the animal experiments when patches were tested that contained glycerol as a plasticizer (Chapter 8). The addition of a plasticizer agent to biodegradable blend films represents a feasible approach to enhance the pliability of biopolymer-based membranes ${ }^{49}$. After the observed effects of the patches, additional analyses identified glycerol as the cause of adverse patch-host reactions. In our cell culture 
experiment, high percentages of glycerol had an obvious harmful effect on adiposederived stem cells. In addition, a chorioallantoic membrane assay showed that the materials evoked a negative biological response leading to tissue injury, characterized by vessel damage and bleeding. Therefore, we advocate that caution should be taken when selecting glycerol as plasticizer in biomaterials designed for intra-abdominal use. Safety should always be the main priority when new materials are being developed, but is of particular importance when these materials intervene with complex biological processes. As stated in the introduction of this thesis, the so-called collagenous equilibrium (the balance between collagen synthesis and lysis) is critical to anastomotic healing. It has been shown that certain anti-adhesive products may predispose to peritonitis and anastomotic dehiscence ${ }^{50,51}$. In this thesis, a newly developed anti-adhesive material was placed in both the presence of a colonic anastomosis as well as in a model for controlled infection to evaluate both efficacy and safety of this potential adhesion barrier (Chapter 9). Poly(trimethylene carbonate) (PTMC), an inert, slow degrading polymer was shown to have excellent biocompatibility and to be phagocytised by macrophages, enabling it to be completely degraded at 12 weeks with only a small area of inflammatory cells that was observed at the site of implantation ${ }^{52,53}$. Unlike other membranous materials, such as collagen membranes, this polymer degrades through surface degradation, allowing it to retain its mechanical characteristics throughout the degradation process ${ }^{54}$.

In both animal models to evaluate the safety of PTMC, presence of the film did not cause any increase in AL rate, nor did it aggravate the induced infection. Prevention of intraperitoneal adhesions remains an integral part of daily surgery and new materials are being developed that may reduce the devastating impact of adhesions on quality of life and diminish the risk for reoperations. However, it should always be taken into account that it is all a matter of balance between anastomotic healing and adhesion formation, which remains a complicated, mysterious process where interventions can have detrimental effects.

\section{Future perspectives and recommendations}

Despite important improvements in perioperative care and increased awareness of $\mathrm{AL}$, incidence rates of this dreaded complication have remained stable over the past decades. Most strikingly, leakage continues to occur in patients treated under the most expert care, without the presence of any known risk factors ${ }^{55}$. The lack of knowledge regarding the pathophysiological process of AL and the process of normal intestinal healing hampers the development of novel predictive and preventive methods. AL has significant impact on morbidity and mortality, quality of life, health care costs and is suggested to negatively interfere with oncological prognosis $5,6,56$. Even though large numbers of clinical trials have identified 


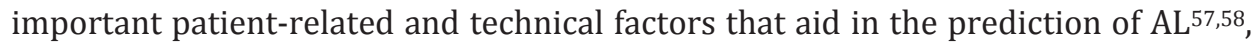
the search for a predictive biomarker for AL has hitherto remained unsuccessful.

Therefore, we have designed a study that focuses on predictive factors for anastomotic leakage (REVEAL-study) after colorectal surgery. This prospective, observational study is based on the hypothesis that AL is partly due to patientderived factors such as a derailed immune response, genetical predisposition and a deficient microbiota composition, and that the clinical course can be further influenced by surgical stress, ischemia and a compromised systemic response. This study aims at 1) broadening our understanding of the pathophysiological process of AL by introducing novel biomarkers of intestinal damage and 2) decreasing the clinical burden of AL by individual risk assessment in the future. Study enrolment of 588 patients undergoing colorectal surgery with the creation of an anastomosis has started in the fall of 2015 and is currently on-going (Clinicaltrials.gov NCT02347735).

In order to minimize the potentially life threatening clinical consequences of a leak, some surgeons opt to perform a deviating stoma directly following the initial operation $^{31}$. Although it is generally accepted that the presence of a colostomy or ileostomy reduces the sequelae of $\mathrm{AL}$ and the need for re-intervention (surgery) in case of a leak 59,60 a decrease in incidence of postoperative mortality has not yet been proven ${ }^{61,62}$. The presence of selection bias, where an ostomy is performed in high-risk patients, should be considered when interpreting these results. Surgeons perform elective deviating ostomy in about $70 \%$ of cases of low rectal carcinoma, of which a significant percentage (19-40\%) will never have their temporary ostomy reversed ${ }^{63,64}$. Possible benefits of a deviating stoma should be weighed against stoma-related morbidity, the impact on quality of life and the mortality rates after stoma closure65,66. An adequate preoperative risk analysis could aid surgeons and their patients in the decision making process regarding the construction of temporary ostomies.

There are already some prediction measures available for clinical practice, however these are designed to detect anastomotic leakage in an early stage, such as the DULK score or the PREDICS study67,68. Both scores are based on clinical markers, indicating a 'sick' patient at risk of having AL. Moreover, these studies also suggest using their scores as a tool for safe and early discharge after elective colorectal surgery, just as the IMACORS study ${ }^{69}$. In this study procalcitonin (PCT) and C-reactive protein (CRP) was studied and showed that CRP is more accurate than PCT for the detection of infectious complications. However, their conclusion is not only based on the AL rate, but on all postoperative infectious complications. The authors from the PREDICS study demonstrated that both PCT and CRP have a good negative predictive value for AL both in $3^{\text {rd }}$ and $5^{\text {th }}$ postoperative day ${ }^{68}$, which was also shown by Reisinger et al. ${ }^{70}$. CRP is already a commonly used marker in the clinical setting, but the use of aforementioned scores is not standard practice yet. 
Currently, there are still studies enrolling patients to develop an optimal diagnostic algorithm for early detection of AL, indicating that the optimal scoring method still has not been found ${ }^{71}$. Perhaps the Dutch Taskforce on Anastomotic Leakage can play a role in analysing which score is most accurate for the detection of AL and implement one of these scores into the Dutch guidelines for postoperative care after colorectal surgery.

Recently, a new prognostic index called PROCOLE was designed to predict the risk of a certain individual developing $\mathrm{AL}$ after colorectal surgery ${ }^{72}$. The big difference with our REVEAL study is that the PROCOLE is solely based on potential risk factors already described in literature and is therefore - until now - only a theoretical index, not taken individual measurements into account.

The successful implementation of risk assessment tools that can be the result from the REVEAL-study would have a positive influence on morbidity and mortality rates, duration of hospital stay, number of readmissions, re-interventions and admissions to the ICU, leading to a significant increase in quality of life for the general patient population ${ }^{57}$.

In addition to the clinical focus on risk factors and individual risk profiling, the main goal for researchers in the experimental field of colorectal anastomosis should be to completely elucidate the healing process and the multifactorial pathophysiology that leads to anastomotic leakage. In the future there should be an interplay between clinical findings and experimental results that will continuously influence one another; observed risk factors in the clinic should be further investigated with new techniques in the lab and potential key factors in anastomotic healing should be identified in the patient population. Only when these two approaches are combined, without aiming directly at reducing AL rates, it will lead to a substantial contribution to the field of gastrointestinal research.

\section{Main conclusion}

Numerous studies have focused on the effect of a certain intervention on the AL rate, without studying the biochemical process of anastomotic healing first. An international summit on intestinal $\mathrm{AL}$ concluded that research into the pathogenesis of AL could be advanced markedly by performing additional analyses in human anastomotic tissues during and after surgery ${ }^{73}$. The use of human tissue will lead to a reduced demand of experimental research, thereby reducing the numbers of animals that are being used nowadays. Furthermore, with current technologies, we are capable of replacing animals with, for example, organoids to investigate the molecular process of intestinal healing in more detail or to use a reduced amount of animals since high resolution mass-spectrometry imaging techniques or digital holographic microscopy can provide more information for the assessment of gastrointestinal wound healing ${ }^{74-76}$. 
We have argued that the ill-defined pathogenesis of AL is a direct consequence from the largely unexplained, complicated biological process of anastomotic healing. Anastomotic healing should be completely elucidated in order to develop interventions that may stimulate anastomotic healing and subsequently prevent AL. New studies should focus on identifying risk factors for AL before starting large randomized controlled trials for specific interventions. This can positively influence the quality and decrease the quantity of animal research on AL in the short term and hopefully reduce the clinical burden of AL in the long term. 


\section{References}

1. Nederland IK. Cijfers over kanker [Internet]. Available from: http://www.cijfersoverkanker.nl/ incidentie-sterfte-50.html

2. Giesbers H, Verweij A, de Beer J. Vergrijzing: Wat zijn de belangrijkste verwachtingen voor de toekomst? [Internet]. Volksgezondheid Toekomst Verkenning, Nationaal Kompas Volksgezondheid. Bilthoven RIVM. Available from: http://www.nationaalkompas.nl/bevolking/vergrijzing/ toekomst/

3. Spanjersberg WR, van Sambeeck JDP, Bremers A, Rosman C, van Laarhoven CJHM. Systematic review and meta-analysis for laparoscopic versus open colon surgery with or without an ERAS programme. Surg Endosc. 2015;29(12):3443-3453.

4. Walker KG, Bell SW, Rickard MJFX, Mehanna D, Dent OF, Chapuis PH, et al. Anastomotic Leakage Is Predictive of Diminished Survival After Potentially Curative Resection for Colorectal Cancer. Ann Surg. 2004;240(2):255-259.

5. Branagan G, Finnis D. Prognosis After Anastomotic Leakage in Colorectal Surgery. Dis Colon Rectum. 2005;48(5):1021-1026.

6. Law WL, Choi HK, Lee YM, Ho JWC, Seto CL. Anastomotic leakage is associated with poor long-term outcome in patients after curative colorectal resection for malignancy. J Gastrointest Surg. 2007;11(1):8-15.

7. Thompson SK, Chang EY, Jobe BA. Clinical review: Healing in gastrointestinal anastomoses, part I. Microsurgery. 2006;26(3):131-136.

8. Thornton FJ, Barbul A. Healing in the gastrointestinal tract. Surg Clin North Am. 1997;77(3):549573.

9. Ågren MS, Andersen TL, Mirastschijski U, Syk I, Schiødt CB, Surve V, et al. Action of matrix metalloproteinases at restricted sites in colon anastomosis repair: an immunohistochemical and biochemical study. Surgery. 2006;140(1):72-82.

10. Ågren MS, Andersen TL, Andersen L, Schiødt CB, Surve V, Andreassen TT, et al. Nonselective matrix metalloproteinase but not tumor necrosis factor- $\alpha$ inhibition effectively preserves the early critical colon anastomotic integrity. Int J Colorectal Dis. 2010;26(3):329-337.

11. Wu Z, Vakalopoulos KA, Boersema GSA, Kroese LF, Lam KH, van der Horst PH, et al. The prevention of colorectal anastomotic leakage with tissue adhesives in a contaminated environment is associated with the presence of anti-inflammatory macrophages. Int J Colorectal Dis. 2014;29(12):1507-1516.

12. Shogan BD, Belogortseva N, Luong PM, Zaborin A, Lax S, Bethel C, et al. Collagen degradation and MMP9 activation by Enterococcus faecalis contribute to intestinal anastomotic leak. Sci Transl Med. 2015;7(286):286ra68-8.

13. van Praagh JB, de Goffau MC, Bakker IS, Harmsen HJM, Olinga P, Havenga K. Intestinal microbiota and anastomotic leakage of stapled colorectal anastomoses: a pilot study. Surg Endosc. 2016;30(6):2259-65

14. Linstone HA, Turoff M. The Delphi Method. Techniques and applications. 2002.

15. NC3Rs Reporting Guidelines Working Group. Animal research: reporting in vivo experiments: the ARRIVE guidelines. J Physiol. 2010;588:2519-21

16. Lipska MA, Bissett IP, Parry BR, Merrie AEH. Anastomotic leakage after lower gastrointestinal anastomosis: men are at a higher risk. ANZ J Surg. 2006;76(7):579-85.

17. Pommergaard HC, Gessler B, Burcharth J, Angenete E, Haglind E, Rosenberg J. Preoperative risk factors for anastomotic leakage after resection for colorectal cancer: a systematic review and meta-analysis. Colorectal Disease. 2014;16(9):662-671.

18. Eberl $\mathrm{T}$, Jagoditsch $\mathrm{M}$, Klingler $\mathrm{A}$, Tschmelitsch J. Risk factors for anastomotic leakage after resection for rectal cancer. Am J Surg. 2008;196(4):592-598.

19. Kang CY, Halabi WJ, Chaudhry 00, Nguyen V, Pigazzi A, Carmichael JC, et al. Risk Factors for Anastomotic Leakage After Anterior Resection for Rectal Cancer. JAMA Surg. 2013;148(1):65-71.

20. Bertelsen CA, Andreasen AH, Jørgensen T, Harling H. Anastomotic leakage after anterior resection for rectal cancer: risk factors. Colorectal Disease. 2010;12(1):37-43. 
21. Slieker JC, Komen N, Komen NAP, Mannaerts GH, Karsten TM, Willemsen P, et al. Long-term and perioperative corticosteroids in anastomotic leakage: a prospective study of 259 left-sided colorectal anastomoses. Arch Surg. 2012;147(5):447-52.

22. Hakkarainen TW, Steele SR, Bastaworous A, Dellinger EP, Farrokhi E, Farjah F, et al. Nonsteroidal anti-inflammatory drugs and the risk for anastomotic failure: a report from Washington State's Surgical Care and Outcomes Assessment Program (SCOAP). JAMA Surg. 2015;150(3):223-8.

23. Grootjans J, Hundscheid IHR, Lenaerts K, Boonen B, Renes IB, Verheyen FK, et al. Ischaemiainduced mucus barrier loss and bacterial penetration are rapidly counteracted by increased goblet cell secretory activity in human and rat colon. Gut. 2013;62(2):250-8.

24. Birchenough GMH, Johansson ME, Gustafsson JK, Bergström JH, Hansson GC. New developments in goblet cell mucus secretion and function. Mucosal Immunol. 2015;8(4):712-719.

25. Binnebösel M, Junge K, Kaemmer DA, Krones CJ, Titkova S, Anurov M, et al. Intraperitoneally applied gentamicin increases collagen content and mechanical stability of colon anastomosis in rats. Int J Colorectal Dis. 2009;24(4):433-440.

26. Pasternak B, Rehn M, Andersen L, Ågren MS, Heegaard A-M, Tengvall P, et al. Doxycycline-coated sutures improve mechanical strength of intestinal anastomoses. Int J Colorectal Dis. 2007;23(3):271-276.

27. Besten den G, van Eunen K, Groen AK, Venema K, Reijngoud D-J, Bakker BM. The role of short-chain fatty acids in the interplay between diet, gut microbiota, and host energy metabolism. J Lipid Res. 2013;54(9):2325-2340.

28. Wen L, Ley RE, Volchkov PY, Stranges PB, Avanesyan L, Stonebraker AC, et al. Innate immunity and intestinal microbiota in the development of Type 1 diabetes. Nature. 2008;455(7216):1109-1113.

29. Uronis JM, Mühlbauer M, Herfarth HH, Rubinas TC, Jones GS, Jobin C. Modulation of the Intestinal Microbiota Alters Colitis-Associated Colorectal Cancer Susceptibility. PLoS ONE. 2009;4(6):e6026.

30. Manichanh C, Borruel N, Casellas F, Guarner F. The gut microbiota in IBD. Nat Rev Gastroenterol Hepatol. 2012;9(10):599-608.

31. Shogan BD, Carlisle EM, Alverdy JC, Umanskiy K. Do we really know why colorectal anastomoses leak? J Gastrointest Surg. 2013;17(9):1698-1707.

32. Ha GW, Kim HJ, Lee MR. Transanal tube placement for prevention of anastomotic leakage following low anterior resection for rectal cancer: a systematic review and meta-analysis. Ann Surg Treat Res. 2015;89(6):313-318.

33. Morks AN, Havenga K, Ploeg RJ. Can intraluminal devices prevent or reduce colorectal anastomotic leakage: a review. World J Gastroenterol. 2011;17(40):4461-4469.

34. Pommergaard H-C, Achiam MP, Rosenberg J. External coating of colonic anastomoses: a systematic review. Int J Colorectal Dis. 2012;27(10):1247-1258.

35. Thibault R, Blachier F, Darcy-Vrillon B, de Coppet P, Bourreille A, Segain J-P. Butyrate utilization by the colonic mucosa in inflammatory bowel diseases. Inflamm Bowel Dis. 2010;16(4):684-695.

36. Bloemen JG, Schreinemacher MH, de Bruine AP, Buurman WA, Bouvy ND, Dejong CH. Butyrate enemas improve intestinal anastomotic strength in a rat model. Dis Colon Rectum. 2010;53(7):1069-75.

37. Aya KL, Stern R. Hyaluronan in wound healing: rediscovering a major player. Wound Repair Regen. 2014;22(5):579-593.

38. Vakalopoulos KA, Daams F, Wu Z, Timmermans L, Jeekel JJ, Kleinrensink G-J, et al. Tissue adhesives in gastrointestinal anastomosis: a systematic review. J Surg Res. 2013;180(2):290-300.

39. Kotzampassi K, Eleftheriadis E. Tissue sealants in endoscopic applications for anastomotic leakage during a 25-year period. Surgery. 2015;157(1):79-86.

40. Nordentoft T, Pommergaard HC, Rosenberg J, Achiam MP. Fibrin Glue Does Not Improve Healing of Gastrointestinal Anastomoses: A Systematic Review. Eur Surg Res. 2015;54(1-2):1-13.

41. Wu Z, Boersema GSA, Vakalopoulos KA, Daams F, Sparreboom CL, Kleinrensink G-J, et al. Critical analysis of cyanoacrylate in intestinal and colorectal anastomosis. J Biomed Mater Res Part B Appl Biomater. 2013;102(3):635-642.

42. Wu Z, Vakalopoulos KA, Kroese LF, Boersema GSA, Kleinrensink GJ, Jeekel J, et al. Reducing Anastomotic Leakage by Reinforcement of Colorectal Anastomosis with Cyanoacrylate Glue. Eur Surg Res. 2013;50(3-4):255-61. 
43. Vakalopoulos KA, Wu Z, Kroese LF, van der Horst PH, Lam KH, Dodou D, et al. Clinical, mechanical, and immunohistopathological effects of tissue adhesives on the colon: An in-vivo study. J Biomed Mater Res B Appl Biomater. 2016 Jan 24. doi: 10.1002/jbm.b.33621.

44. Bae K-B, Kim S-H, Jung S-J, Hong K-H. Cyanoacrylate for colonic anastomosis; is it safe? Int J Colorectal Dis. 2010;25(5):601-606.

45. Bicer YO, Koybasi S, Seyhan S, Firat T, Kazaz H. Outcomes from Cyanoacrylate Use in Tracheal Anastomosis in a Rat Model. Otolaryngol Head Neck Surg. 2015;153(5):815-821.

46. Fortelny RH, Petter-Puchner AH, Walder N, Mittermayr R, Öhlinger W, Heinze A, et al. Cyanoacrylate tissue sealant impairs tissue integration of macroporous mesh in experimental hernia repair. Surg Endosc. 2007;21(10):1781-1785.

47. Paral J, Subrt Z, Lochman P, Klein L, Hadzi-Nikolov D, Turek Z, et al. Suture-Free Anastomosis of the Colon Experimental Comparison of Two Cyanoacrylate Adhesives. J Gastrointest Surg. 2011;15(3):451-9.

48. Scognamiglio F, Travan A, Rustighi I, Tarchi P, Palmisano S, Marsich E, et al. Adhesive and sealant interfaces for general surgery applications. J Biomed Mater Res Part B Appl Biomater. 2015 Apr 17;104(3):626-639.

49. Vieira MGA, da Silva MA, Santos dos LO, Beppu MM. Natural-based plasticizers and biopolymer films: A review. European Polymer Journal. 2011;47(3):254-263.

50. Fazio VW, Cohen Z, Fleshman JW, van Goor H, Bauer JJ, Wolff BG, et al. Reduction in adhesive smallbowel obstruction by Seprafilm adhesion barrier after intestinal resection. Dis Colon Rectum. 2006 ;49(1):1-11.

51. Tang C-L, Jayne DG, Seow-Choen F, Ng Y-Y, Eu K-W, Mustapha N. A Randomized Controlled Trial of $0.5 \%$ Ferric Hyaluronate Gel (Intergel) in the Prevention of Adhesions Following Abdominal Surgery. Ann Surg. 2006;243(4):449-455.

52. Pêgo AP, Van Luyn MJA, Brouwer LA, van Wachem PB, Poot AA, Grijpma DW, et al. In vivo behavior of poly(1,3-trimethylene carbonate) and copolymers of 1,3-trimethylene carbonate with D,Llactide or epsilon-caprolactone: Degradation and tissue response. J Biomed Mater Res A. 2003;67(3):1044-1054.

53. Van Leeuwen AC, Huddleston Slater JJR, Gielkens PFM, de Jong JR, Grijpma DW, Bos RRM. Guided bone regeneration in rat mandibular defects using resorbable poly(trimethylene carbonate) barrier membranes. Acta Biomater. 2012;8(4):1422-1429.

54. Van Leeuwen AC, Van Kooten TG, Grijpma DW, Bos RRM. In vivo behaviour of a biodegradable poly(trimethylene carbonate) barrier membrane: a histological study in rats. J Mater Sci Mater Med. 2012;23(8):1951-1959.

55. Bellows CF, Webber LS, Albo D, Awad S, Berger DH. Early predictors of anastomotic leaks after colectomy. Tech Coloproctol. 2009;13(1):41-47.

56. Bell SW, Walker KG, Rickard MJFX, Sinclair G, Dent OF, Chapuis PH, et al. Anastomotic leakage after curative anterior resection results in a higher prevalence of local recurrence. Br J Surg. 2003;90(10):1261-1266.

57. Alberts JCJ, Parvaiz A, Moran BJ. Predicting risk and diminishing the consequences of anastomotic dehiscence following rectal resection. Colorectal Disease. 2003;5(5):478-482.

58. Boccola MA, Buettner PG, Rozen WM, Siu SK, Stevenson ARL, Stitz R, et al. Risk factors and outcomes for anastomotic leakage in colorectal surgery: a single-institution analysis of 1576 patients. World J Surg. 2011;35(1):186-195.

59. Matthiessen P, Hallböök O, Rutegård J, Simert G, Sjödahl R. Defunctioning stoma reduces symptomatic anastomotic leakage after low anterior resection of the rectum for cancer: a randomized multicenter trial. Ann Surg. 2007;246(2):207-214.

60. Shukla PJ, Pandey D. Protective defunctioning stoma in low anterior resection for rectal carcinoma (Br J Surg 2005; 92: 1137-1142). Br J Surg. 2005;92(12):1565-1566.

61. Hanna MH, Vinci A, Pigazzi A. Diverting ileostomy in colorectal surgery: when is it necessary? Langenbecks Arch Surg. 2015;400(2):145-152.

62. Anderin K, Gustafsson UO, Thorell A, Nygren J. The effect of diverting stoma on postoperative morbidity after low anterior resection for rectal cancer in patients treated within an ERAS program. Eur J Surg Oncol. 2015;41(6):724-730. 
63. Mealy K, O'Broin E, Donohue J, Tanner A, Keane FB. Reversible colostomy--what is the outcome? Dis Colon Rectum. 1996;39(11):1227-1231.

64. Lim SW, Kim HJ, Kim CH, Huh JW, Kim YJ, Kim HR. Risk factors for permanent stoma after low anterior resection for rectal cancer. Langenbecks Arch Surg. 2013;398(2):259-264.

65. Güenaga KF, Lustosa SAS, Saad SS, Saconato H, Matos D. Ileostomy or colostomy for temporary decompression of colorectal anastomosis. Güenaga KF, editor. Cochrane Database Syst Rev. 2007;(1):CD004647.

66. Mala T, Nesbakken A. Morbidity related to the use of a protective stoma in anterior resection for rectal cancer. Colorectal Disease. 2008;10(8):785-788.

67. Dulk den M, Noter SL, Hendriks ER, Brouwers MAM, van der Vlies CH, Oostenbroek RJ, et al. Improved diagnosis and treatment of anastomotic leakage after colorectal surgery. Eur J Surg Oncol. 2009;35(4):420-426.

68. Giaccaglia V, Salvi PF, Antonelli MS, Nigri G, Pirozzi F, Casagranda B, et al. Procalcitonin Reveals Early Dehiscence in Colorectal Surgery. Ann Surg. 2016;263(5):967-972.

69. Ortega-Deballon P, Facy O, Paquette B, Orry D, Binquet C, Rat P. Diagnostic accuracy of inflammatory markers as early predictors of infection after elective colorectal surgery: results from the IMACORS study. Ann Surg. 2016;263(5):961-966.

70. Reisinger KW, Poeze M, Hulsewé KWE, van Acker BA, van Bijnen AA, Hoofwijk AGM, et al. Accurate Prediction of Anastomotic Leakage after Colorectal Surgery Using Plasma Markers for Intestinal Damage and Inflammation. J Am Coll Surg. 2014;219(4):744-751.

71. Kornmann V, van Ramshorst B, van Dieren S, van Geloven N, Boermeester M, Boerma D. Early complication detection after colorectal surgery (CONDOR): study protocol for a prospective clinical diagnostic study. Int J Colorectal Dis. 2015;31(2):459-464.

72. Rojas-Machado SA, Romero-Simó M, Arroyo A, Rojas-Machado A, López J, Calpena R. Prediction of anastomotic leak in colorectal cancer surgery based on a new prognostic index PROCOLE (prognostic colorectal leakage) developed from the meta-analysis of observational studies of risk factors. Int J Colorectal Dis. 2015;31(2):197-210.

73. Shogan BD, An GC, Schardey HM, Matthews JB, Umanskiy K, Fleshman JW, et al. Proceedings of the first international summit on intestinal anastomotic leak, Chicago, Illinois, October 4-5, 2012. Surg Infect (Larchmt). 2014;15(5):479-489.

74. Sato T, Clevers H. Growing self-organizing mini-guts from a single intestinal stem cell: mechanism and applications. Science. 2013;340(6137):1190-1194.

75. Giesen C, Wang HAO, Schapiro D, Zivanovic N, Jacobs A, Hattendorf B, et al. Highly multiplexed imaging of tumor tissues with subcellular resolution by mass cytometry. Nat Methods. 2014;11(4):417-422.

76. Bettenworth D, Lenz P, Krausewitz P, Brückner M, Ketelhut S, Domagk D, et al. Quantitative stainfree and continuous multimodal monitoring of wound healing in vitro with digital holographic microscopy. PLoS ONE. 2014;9(9):e107317. 
Nederlandse samenvatting 


\section{Nederlandse samenvatting}

In Nederland worden per jaar meer dan 15.500 nieuwe patiënten met colorectaal carcinoom (CRC) gediagnostiseerd. Het was daarmee de meest voorkomende oncologische aandoening in 2015, bij zowel mannen als vrouwen. Het aantal patiënten met CRC zal toenemen door een stijgende incidentie, door de bevolkingsgroei, door de vergrijzing en door het recent gestarte bevolkingsonderzoek. Jaarlijks overlijden 4.000 mensen aan de gevolgen van CRC. Twee derde van de patiënten met CRC ondergaat een operatieve behandeling waarbij de darmtumor verwijderd wordt. Dit blijft ondanks allerlei nieuwe (radio)therapieën de hoeksteen van de behandeling voor colorectaal carcinoom. Het beloop rondom de operatie bij colorectale chirurgie is cruciaal voor de klinische uitkomst met betrekking tot overlijden en functioneel herstel. Daarnaast heeft deze operatieve behandeling een grote impact op de zorgkosten.

In de afgelopen decennia is de perioperatieve zorg verbeterd dankzij ontwikkelingen in de anesthesie, minimaal invasieve operatieve technieken en de introductie van 'fast-track' protocollen welke gericht zijn op een snel postoperatief herstel van de patiënt. Er ontstaan echter nog steeds complicaties na colorectale chirurgie, waarvan naadlekkage de meest gevreesde is. Naadlekkage ontstaat als de twee aan elkaar gemaakte darmuiteinden - de darmnaad oftewel anastomose - niet goed geneest; de darminhoud lekt dan in de buikholte wat kan leiden tot onder andere buikvliesontsteking en abcesvorming.

Bij 712 van de 10.426 (6,8\%) geregistreerde patiënten met een colorectale resectie ontstond in Nederland in 2014 een naadlekkage met een heroperatie tot gevolg (Dutch Surgical Colorectal Audit (DSCA) 2014). Dit ging gepaard met een hoge morbiditeit, verlengd ziekenhuisverblijf en zelfs mortaliteit. Naadlekkage wordt bovendien geassocieerd met verminderde ziekte-specifieke overleving en toegenomen kans op het terugkeren van colorectaal carcinoom. De incidentie van naadlekkage is helaas gelijk gebleven in de afgelopen decennia, dit komt met name door het gebrek aan kennis van een normale naadgenezing (Hoofdstuk 1). Het proces van wondgenezing in de darm, bijvoorbeeld bij het aanleggen van een naad, wordt vaak vergeleken met wondgenezing van de huid. De darmgenezing vindt inderdaad plaats in de drie bekende fasen van ontsteking, proliferatie en remodelling. Er zijn echter ook substantiële verschillen; zo gaat de darmgenezing veel sneller dan de genezing van de huid, zijn er verschillende cellen en stoffen (bijvoorbeeld ander type collageen) bij betrokken en heeft zowel de huid als de darm een unieke, microbiota.

\section{Diermodellen voor onderzoek naar naadlekkage na darmchirurgie}

Aangezien het probleem van naadlekkage helemaal niet minder is geworden in de afgelopen jaren, zijn veel mensen geïnteresseerd in dit mysterie. Daarom er wordt 
er wereldwijd dan ook uitgebreid onderzoek naar gedaan naar dit fenomeen. Vaak worden hiervoor proefdieren gebruikt, waarin een darmnaad gemaakt wordt. Omdat het nog onmogelijk is de darmgenezing op moleculair niveau bij de mens in kaart te brengen wordt ook in de experimenten beschreven in dit proefschrift gebruikt gemaakt van diermodellen.

Niet alle modellen worden even vaak in de literatuur gebruikt. Teneinde meer duidelijkheid over deze modellen en vergelijkbaarheid tussen de verschillende studies te verkrijgen, is het cruciaal dat onderzoekers overeenstemming bereiken over wat belangrijk is bij het onderzoek naar darmnaden. Om die reden hebben we een Delphi-analyse uitgevoerd. Dat is een onderzoeksmethode waarbij de meningen van een groot aantal experts wordt gevraagd en door de antwoorden van de experts anoniem terug te koppelen aan eenieder wordt in een aantal rondes geprobeerd tot consensus te komen. Een van de uitkomsten van deze studie was dat de hond en het konijn geen gevalideerde modellen zijn voor dit soort onderzoek (Hoofdstuk 2). De muis, rat en het varken zijn wel geschikt geacht als diermodel, mits er gewerkt wordt volgens de huidige wet- en regelgeving en de experimenten gerapporteerd worden volgens de ARRIVE-guidelines. Uit deze studie is een nieuwe score gekomen om experimentele anastomoses te kunnen beoordelen. Deze werd door de respondenten als zeer geschikt bevonden, hoewel de bruikbaarheid nog bewezen dient te worden in de praktijk.

Ondanks de hoeveelheid studies die worden uitgevoerd naar het onderwerp naadlekkage, is het nog steeds onduidelijk hoe zo'n naadlekkage eigenlijk ontstaat. We weten niet goed wat er mis gaat bij normale naadgenezing wat leidt tot lekkage van darminhoud. Om het naadgenezingsproces meer in detail in kaart te brengen hebben we gebruik gemaakt van de relatieve nieuwe techniek 'massaspectrometrie imaging' (Hoofdstuk 3). Deze techniek stelt ons in staat om onderscheid te maken tussen de verschillende lagen van de darmwand en specifieke stoffen te identificeren als potentiele aangrijpingspunten om het naadgenezingproces verder te ontrafelen. Dit kan weer tot mogelijke nieuwe interventies, die tot een betere heling en dus een lager percentage naadlekkage in de toekomst leiden.

\section{Risico-factoren opsporen middels specifieke 'knock-out' proefdieren}

Het is bekend dat bepaalde patiëntfactoren het risico op naadlekkage vergroten: zo heeft bijvoorbeeld een oudere man met overgewicht die rookt een groter risico op naadlekkage dan een slanke, niet-rokende dame. Ook is gebleken uit observationeel en experimenteel onderzoek dat patiënten die bepaalde pijnstillers met ontstekingsremmende werking, zogenaamde NSAIDs, kregen rondom de operatie vaker een naadlekkage ontwikkelden. In dit proefschrift is een deel van het mechanisme opgehelderd waarom deze medicijnen bijdragen aan een hoger risico 
op naadlekkage; zo is aangetoond dat remming of depletie van het enzym Cyclooxygenase 2 (COX-2) leidt tot een toename van het aantal naadlekkages in een muismodel, mogelijk door de doorbloeding van de darmnaad te beïnvloeden. COX-2 speelt een grote rol bij de ontstekingsfase van het genezingsproces en is essentieel voor een normale naadgenezing (Hoofdstuk 4).

Verder zijn er aanwijzingen dat een normale samenstelling van de slijmlaag (mucus) in de darm belangrijk is bij de normale naadgenezing. Zo zagen we dat het naadlekkage percentage significant hoger was in muizen die de belangrijkste component van mucus in het colon missen, de zogenoemde Muc2 knockout muizen, dan dat van controle dieren (Hoofdstuk 5). Er zijn verschillende verklaringen voor dit resultaat mogelijk. Een van die verklaringen kan zijn dat door het gebrek aan de mucuslaag in de darmen geen natuurlijke habitat is voor verschillende bacteriën. Bacteriën zijn essentieel om het natuurlijk evenwicht in de darmen te behouden. Een van de taken van de bacteriën in de darm is vezels uit de voeding af te breken naar kleinere deeltjes waar wij energie uit kunnen halen. Bij deze omzetting komen korte-keten vetzuren vrij, stofjes die weer door de darmcellen als voedingsstof gebruikt kunnen worden. Een van die korte-keten vetzuren is butyraat. Dit stofje is al vaker onderzocht in de darm en er is aangetoond dat dit de naad versterkt wanneer dit als klysma wordt gegeven in ratten. In dit proefschrift is getracht een betere methode dan klysma's uit te testen om zo ook darmnaden in het colon hogerop (proximaal) te kunnen versterken, alsmede het butyraatklysma te verrijken met andere stoffen (Hoofdstuk 6). Helaas leverden deze experimenten weinig nieuwe inzichten op en lijkt butyraat suppletie middels klysma toch de beste optie. In de kliniek is men echter terughoudend met het geven van klysma's wanneer een darmnaad is aangelegd. Artsen zijn bezorgd dat het klysma door directe mechanische krachten of via verhoogde spanning op de naad juist kan leiden tot het 'scheuren of knappen' van de naad.

\section{Het verminderen van naadlekkage door innovatieve technieken}

Er zijn veel pogingen gedaan om de naad gedurende de operatie te versterken om naadlekkage te voorkomen. Veel gebruikte middelen hierbij zijn: lijmen, plakstrips of patches bestaande uit biologische materialen. Ook in dit proefschrift is getracht om preventie van naadlekkage middels een lijm of een patch te bereiken. In hoofdstuk 7 onderzochten we verschillende commercieel verkrijgbare lijmen en het effect daarvan op het ontstaan van naadlekkage, stenose en algeheel welzijn van de dieren. Het bleek dat sommige lijmen wel degelijk tot een reductie kunnen leiden van het aantal naadlekkages, maar dat veel lijmen ook een negatief effect hebben op de darmmotiliteit en ongerief veroorzaakten bij de ratten. De optimale lijm die de naad kan verstevigen zonder verder enige reactie te geven is nog niet gevonden. 
Daarom hebben we tevens de optie van een patch onderzocht, dit in het kader van het Europese project 'AnastomoSEAL' waarbij wij, als onderzoeksgroep, verantwoordelijk waren voor de dierexperimenten. Een lastig aspect van dit soort materialen voor intra-abdominaal gebruik is de mate van adhesie: dus hoe goed iets plakt daar de buikholte een redelijk vochtige omgeving is. Daarnaast wordt er toch een materiaal ingebracht in de nabijheid van een complex biologisch proces, waarbij het lastig is te voorspellen wat de reactie zal zijn.

\section{Mogelijke verstoring tussen het evenwicht van adhesievorming en naadgenezing}

Stoffen die veelbelovend lijken in het lab kunnen in een levend wezen heel anders reageren. Hier dient bij het ontwikkelen van biomedische producten rekening mee te worden gehouden Niet alleen moeten de hoofdbestanddelen goed onderzocht zijn, ook de hulpstoffen, want ook deze kunnen tot ongewenste effecten leiden. Zo kan de stof glycerol in bepaalde doseringen toxisch zijn voor cellen van omentum, wat normaal gesproken om de darmnaad heen zit en bijdraagt aan de naadgenezing (Hoofdstuk 8). Voorzichtigheid is dus geboden wanneer het gaat om het gebruik van nieuw ontwikkelde materialen. Naast effectiviteit is veiligheid ook een belangrijk punt voor het testen in een diermodel. Zo zijn adhesies veel voorkomende complicaties na grote buikchirurgie. Er worden veel preventieve middelen ontwikkeld die het ontstaan van adhesies kunnen verminderen. Dit soort stoffen kunnen echter een nadelig effect hebben op de natuurlijke naadgenezing en dus een groter risico op naadlekkage geven. In dit proefschrift is zo'n nieuw antiadhesief materiaal, zowel effectief tegen adhesies als veilig voor de darmnaad bevonden (Hoofdstuk 9).

Helaas is er nog geen acceptabel alternatief gevonden om het effect van dit soort materialen te testen. Er wordt er dus nog steeds gebruik gemaakt van diermodellen. Dit is zeer belangrijk en resultaten hiervan moeten altijd openbaar en beschikbaar zijn voor de wetenschappelijke wereld. Niets is zo verderfelijk als het herhalen van experimenten die al eerder hebben geleid tot het lijden van mens of dier.

\section{Conclusie}

Het proces van naadlekkage na dikke-darm chirurgie blijft tot op heden nog niet opgehelderd. Hierdoor blijken veelbelovende technieken ter preventie van naadlekkage in de klinische praktijk helaas niet succesvol. Wanneer de pathofysiologie van naadlekkage beter in beeld kan worden gebracht kan dit leiden tot nieuwe aangrijpingspunten waarmee in de toekomst het genezingsproces van de darmnaad kan worden verbeterd en daarmee naadlekkage kan worden 
voorkomen. Dit zal op de langere termijn leiden tot minder morbiditeit en mortaliteit na colorectale chirurgie. 
Valorisation addendum 


\section{Valorisation addendum}

Valorisation is often defined as the process of value creation from knowledge, by making it applicable and available for economic or societal utilisation, and by translating it in the form of new business, products, services or processes ${ }^{1}$.

The aim of this chapter is to take a brief look upon the return of investment society has received from the knowledge gathered during this PhD trajectory. As the work in this thesis largely focuses on experimental animal studies, it is evident that not all results can directly be translated to the clinical setting. Here the translation of our academic findings regarding possibilities for future implementation and economic/societal value will be discussed.

\section{Economical relevance}

This thesis focused on the problem of anastomotic leakage, a severe complication after colorectal surgery. The burden to society of colorectal cancer surgery can significantly be reduced by implementation of the knowledge obtained from this project for several reasons. In the Netherlands approximately 10.000 oncological colorectal operations are performed each year ${ }^{2}$. With an incidence in anastomotic leakage of about $15 \%$, this implies 1500 cases. Notably, the incidence of colorectal carcinoma is expected to rise in the coming years due to national screening programs, increasing the frequency of colorectal cancer treatable by surgery and people becoming older.

We estimate that a reduction of $30-40 \%$ in anastomotic leakage incidence can be achieved by combining perioperative innovations - some of which have their basis in this thesis -, preventing complications in approximately 500 patients yearly in the Netherlands. With a mortality of $15 \%$, roughly 75 deaths can be prevented each year. Moreover, a decrease in intensive care unit admissions can reduce national healthcare costs dramatically. Additional significant contributions to the cost buildup that can be decreased are prolonged hospitalization, extra radiographic studies during and after admission, more consultation services, more extended use of the operating room, stoma-related complications, re-intervention for ostomy closure, longer nursing care, materials (e.g. antibiotics, ostomy bags) ${ }^{3}$. It has been estimated that the actual cost per patient increased fivefold in case of serious anastomotic leakage (in patients who did not receive a diverting stoma) ${ }^{4-5}$.

No attempt has been made in the Netherlands to assess the total cost involved for a single case of colorectal leak. This largely due to the fact that hospitals do not have a scale of charges for individual consultations and services involved. Moreover, the costs of rehabilitation, physiotherapy, prolonged absence from work 
of patients and family members (i.e. caregivers), and nursing homes must be considered.

\section{Societal relevance}

Not only would a solution to anastomotic leakage be beneficial for the economy, it also has significant advantages for patients who undergo colorectal surgery; as anastomotic leakage is associated with high disability, diminished quality of life and a potential worse oncological outcome6,7. In 2008 a study was published that describes how patients receiving a stoma after colorectal surgery had poorer quality of life, lower body image, poor social activity and also had significantly higher depression and anxiety ${ }^{8}$. All these morbidities can be explained by the fact that these patients face problems including adapting to the new anatomy, managing the stoma and continuing normal activities in their socio-cultural environment. Also, work productivity of these patients decreases significantly due to the level of disability related to multiple abdominal operations or presence of a stoma. Finally, anastomotic leakage itself has an adverse effect on postoperative health-related quality of life when compared to patients that also received a stoma, but did not develop anastomotic leakage postoperatively ${ }^{9}$.

\section{Scientific relevance}

As stated in the introduction of this thesis, unraveling the healing process of bowel anastomosis and identifying local molecular and biochemical responses that disturb healing is scientifically relevant in itself. In depth studies into this topic are essential in order to develop effective strategies and agents to combat healing disturbance and to prevent leakage. Knowledge gained on disturbed bowel anastomotic healing can be tested on other intestinal anastomoses, such as pancreatic and esophageal anastomoses, that are known to frequently leak. In this thesis relative new techniques such as mass spectrometry were used in a pilot study and this study will be able to define the value of mass spectrometry in identifying biochemical processes in the gut wall. This may reveal data relevant for other pathophysiological processes, such as cancer development and inflammatory bowel disease and diverticulitis. In addition to new techniques, relatively new animal models were used to investigate leakage prevention.

\section{Use of animal models}

The studies performed during this $\mathrm{PhD}$ thesis focused on risk factors of anastomotic leakage on the one hand and on potential preventive interventions on the other hand. The latter was extensively studied in the European AnastomoSEAL project $^{10}$. The AnastomoSEAL Consortium worked on the development of a new 
product to prevent anastomotic leakage. AnastomoSEAL was produced as a biocompatible, resorbable sealant patch that was applied around the site of anastomosis; unfortunately this project did not reach its final goal, since experimental studies showed unexpected adverse events. The use of animal models during the development of new biomaterials for human use is irreplaceable and crucial since the effect of biomaterials in vivo are a complex, dynamic process that sadly cannot be investigated in vitro yet.

In this thesis we have reached consensus on animal models specifically designed for research into bowel anastomoses of the lower gastrointestinal tract (Chapter 2). One of the main conclusions was that rabbit and dog models should be abandoned in this type of research. Furthermore, more standardization can be reached by following the ARRIVE guidelines ${ }^{11}$. Experienced researchers in the field are convinced that this provides more transparency and more comparability between studies. In addition, experts on intestinal anastomotic leak have held an International Summit (first in 2012, recently in 2016) where the majority of the respondents consider that current experimental animal models of anastomotic leakage are useful and should continue ${ }^{12}$. However, they also advocate the use of human anastomotic tissues for more analytical research and promote clinical studies to define and characterize the biological variables that are associated with anastomotic healing versus leakage. The REVEAL study (started Summer 2015 in Maastricht) aims to establish and validate a diagnostic algorithm for the preoperative prediction of AL using a combination of inflammatory, immune-related and genetic parameters ${ }^{13}$. With the results from the REVEAL study, the recognition of patients who are at risk for $\mathrm{AL}$ is expected to increase; subsequently recommendations for patients at risk can be made regarding deviating stomas and possible preventive strategies. Lastly, the REVEAL study aims to develop an algorithm for post-operative diagnosis of $\mathrm{AL}$ at an earlier stage, which will positively reflect on short-term survival rates.

\section{Perspective on knowledge utilisation}

During the work for this thesis, collaboration with several national and international scientists has been established. These partnerships are crucial to accelerate research, improve transparency between research centres (Rotterdam \& Nijmegen) and to direct cross-fertilize, which leads to innovation. Furthermore, it can be considered irrational that in a relative small country as the Netherlands researchers are investigating the same topics, unintentionally delaying work - not to mention wasting time and money - that may lead to the solution of AL; an important health problem that is too extensive to be studied by a single researcher. Therefore, the taskforce anastomotic leakage was re-established (as part of the work group Coloproctology), with the purpose to provide more transparency in 
current experimental research, national registration of patients with $\mathrm{AL}$ and increase collaboration regarding the conduction of clinical studies ${ }^{14}$. This is in line with one of the aims of the Dutch Organization for Scientific Research (NWO): that researchers should no longer work against each other, back to back, but instead work together, side-by-side ${ }^{15}$. 


\section{References}

1. Report 'Van voornemens naar voorsprong: kennis moet circuleren - voorstel voor een Nederlandse valorisatieagenda (Waas, 2010).

2. Dutch Surgical Colorectal Audit, Jaarrapportage 2014: uitkomst van zorg registratie. Retrieved from: www.clinicalaudit.nl/dsca

3. Hammond J, Lim S, Wan Y, Gao X, Patkar A. The Burden of Gastrointestinal Anastomotic Leaks: an Evaluation of Clinical and Economic Outcomes. J Gastrointest Surg. 2014 Mar 27;18(6):1176-1185.

4. Vonlanthen R, Slankamenac K, Breitenstein S. et al. The implications of complications on costs of major surgical procedures: a cost analysis of 1200 patients. Ann Surg. 2011;254:907-913

5. Koperna T. Cost-effectiveness of defunctioning stomas in low anterior resections for rectal cancer: a call for bench-marking. Arch Surg 2003;138:1334-1339.

6. Hallbook 0, Sjodahl R. Anastomotic leakage and functional outcome after anterior resection of the rectum. Br J Surg 1996;83:60-62

7. Mirnezami A, Mirnezami R, Chandrakumaran K, Sasapu K, Sagar P, Finan P. Increased Local Recurrence and Reduced Survival From Colorectal Cancer Following Anastomotic Leak. Ann of Surg, 2011;253(5):890-899.

8. Zajac 0, Spychala A, Murawa D. Quality of life assessment in patients with a stoma due to rectal cancer. Reports of Practical Oncology \& Radiology. 2008;13(3):130-134.

9. Marinatou, A., Theodoropoulos, G.E., Karanika, S., Karantanos, T., Siakavellas, S., Spyropoulos, B.G. Do anastomotic leaks impair postoperative health-related quality of life after rectal cancer surgery? A case-matched study. Dis Colon Rectum. 2014;57(2):158-66.

10. Donati I. The AnastomoSEAL Project. Retrieved from: http://www.anastomoseal.eu

11. NC3Rs Reporting Guidelines Working Group. Animal research: reporting in vivo experiments: the ARRIVE guidelines. J Physiol. 2010;588:2519-21.

12. Shogan, B. D., An, G. C., Schardey, H. M., Matthews, J. B., Umanskiy, K., Fleshman, J. W., et al. Proceedings of the first international summit on intestinal anastomotic leak, Chicago, Illinois, October 4-5, 2012. Surg Infect (Larchmt). 2014;15(5):479-89

13. Jongen A.C., Bosmans J.W., Kartal S., Lubbers T., Sosef M., Slooter G.D., Stoot J.H., van Schooten F.J., Bouvy N.D., Derikx J.P. Predictive factors for anastomotic leakage after colorectal surgery: The REVEAL-study. JMIR Res Protoc. 2016 Jun 9;5(2):e90

14. Croonen H. Nieuw initiatief tegen Naadlekkage Nieuwsbrief Medisch Contact, retrieved from: http://medischcontact.artsennet.nl/Actueel/Nieuws/Nieuwsbericht/149828/Initiatief-tegennaadlekkage.htm

15. NWO Strategie 2015-2018. Retrieved from www.nwo.nl 
Dankwoord 


\section{Dankwoord}

Hoewel ik in het echte leven vol enthousiasme eindeloze woorden kan spuien, vind ik het op papier toch wat lastiger. Dit wil niet zeggen dat ik niet ontzettend dankbaar ben voor alle hulp, steun en gezelligheid die ik tijdens mijn promotie-tijd heb mogen ontvangen. Hopelijk komt het met weinig woorden toch goed over en zo niet, dan weet je het wel als ik je persoonlijk met een echte knuffel bedank!

Allereerst natuurlijk mijn promotor en co-promotor, ik had met geen ander team dit willen meemaken....

Prof. Bouvy, beste Nicole, als jij niet m'n promotor was geweest had ik nooit zo'n leuke tijd gehad! Altijd welkom om even binnen te lopen, even te bellen, een smsje als het dringend is en een what's appje voor de leuke foto's. Zelfs bij serieuze onderzoeksbesprekingen is er altijd tijd voor een grapje en ik ken niemand die meer geïnteresseerd is in het privéleven van alle onderzoekers en assistenten dan jij. En dan te bedenken dat het waardevolste wat ik heb geleerd van jou is om te weten wanneer je je mond moet houden ;)

Ik hoop dat we in de toekomst nog veel zullen samenwerken en ben je erg dankbaar voor alle mogelijkheden die je me hebt gegeven de afgelopen jaren!

Dr. Derikx ofwel Professor Joep, ook al ben je dan officieel nog geen professor, aan je kennis, kunde en inzet zal het niet liggen. Jij bent de meest enthousiaste dokter die ik ken, zowel over klinische zaken als over het onderzoek. Het is inspirerend om te zien wat voor pretoogjes je krijgt als je me weer eens inlicht over je meest recente plannen. Het is een eer dat je een rol voor mij daarin ziet. Ik weet zeker dat door jouw onderzoek patiënten betere zorg krijgen en dat je nog vele jaren succesvol zult zijn in alles wat je doet! Goed dat je als echte Limbo nu geniet van het Amsterdamse :)

De leden van mijn promotiecommissie, allereerst natuurlijk Prof. Stassen, bedankt voor het vertrouwen in zowel mijn wetenschappelijke werk als klinische potentie. Prof. van Goor, dank u wel voor de samenwerking, aan de ideeën zal het niet liggen, jammer dat de tijd en moeite (nog) niet heeft geresulteerd in de financiële boost waar we op gehoopt hadden. Dr. Roumen en Dr. Jonkers, helaas nog niet veel intensief samengewerkt, maar daardoor kon $u$ wel in de beoordelingscommissie plaatsnemen: heel veel dank voor het lezen en kritisch beoordelen van dit manuscript. Daarnaast in het bijzonder Steven, we hebben flinke discussies gehad, maar je regelt het allemaal toch maar! Dat het lab mag groeien en bloeien tot in de lengte der dagen... 
Alle co-auteurs die hun bijdrage hebben gelegen aan de artikelen in dit proefschrift en daarbuiten, jullie inbreng heeft zeker bijgedragen aan de kwaliteit ervan!

Lieve David, Frans, Irene-Fleur, Martine, Kim, Kiran \& Junfang, zonder jullie als harde kern was mijn promotietijd nooit zo gezellig geweest; van Thembi tot samen wielrennen, van pubquizzen tot postprandiale puzzelpauzes, van de Efteling tot eindeloze etentjes. Woorden zijn niet genoeg om te zeggen hoeveel ik aan jullie gehad heb, individueel, met aanhang (Sophie :) ) of met z'n allen, ik ga jullie missen!

0ddrie oftewel Audrey, we leerden elkaar snel kennen toen jij mijn semi-arts was (en we in 1 bed sliepen; dat helpt ook) maar had geen fijnere directe collega kunnen wensen! Toch duidelijk te zien he, als je in de horeca gewerkt hebt ;) Weet zeker dat we nog veel contact gaan houden al is het maar om de REVEAL tot een groot succes te maken! Ga zo door, dan komt het vast helemaal goed en switchen mag altijd hoor, oftalmologie is niet eens uit te spreken!

Leo-leooooooo (iedereen komt als je Leo roept), Luukster, medebrabo van me (alleen de rood-witte kleurencombi is mooi aan PSV) en Claire-what's-up-with-thehair: volgens mij heb ik inmiddels met bijna iedereen van het lab wel een kamer gedeeld, maar gezien de jaloezie-app - laten we in het midden houden wie nou wel/niet jaloers was op wie - en het feit dat jullie bij mijn laatste meest stressvolle periode waren (thanx voor alle printjes!) + mijn chocolade/limo/ koffievoorraad zonder gene leegroofden - zie ik jullie als mn ware roomies: weet zeker dat jullie mij gaan missen! Ik jullie natuurlijk ook en we gaan elkaar zeker weer in de kliniek tegenkomen, hẻnnige spoesjkoppen!

Selwynovitch, van Rijn, ga je lekkah? Even bakkie doen binnenkort als je niet te drukdrukdruk bent? Weet zeker dat je harde werken je gaat brengen waar je wilt zijn!

Kostanjos, gekke irritante vent die je bent, wie houdt er niet van jou? Bedankt voor de supervisie tijdens m'n semistage, het zwart-op-wit zetten van mijn bekendste uitspraak en de serieuze en vooral ook niet-serieuze dingen die ik van je geleerd heb en nog steeds leer, tot snel he!

Dirkster, ben jou en Kostan nog steeds dankbaar dat jullie me in de kelder hebben achtergelaten hoewel ik destijds alleen maar kon huilen dat ik in mn uppie met die knaagdieren zat. De enige manier om iets te leren is om het zelf te doen, daar heb je helemaal gelijk in, maar een experiment door jou te laten voordoen is 
een goede 2e! Hoop dat jouw nauwkeurigheid je binnen de chirurgie tot grote hoogtes brengt.

Dennis met je vieze praatjes, Inca met je vieze praatjes - stoer van buiten, lief van binnen ;) Later tijdens ons gezamenlijk ANIOSsen werd dat meer dan eens duidelijk! Ben trots op je dat je je hart gevolgd hebt en als ik niet uit iets microscopisch kom ben jij de $1^{\text {e }}$ die ik bel! Mo met de bulderlach - altijd leuk om die te horen, Bas - "het ligt echt niet aan mij!" Goeie gesprekken waren dat ;) Annemarie ELISA-queen, jullie als 'oude poep' met de vroege lunch hebben mij als guppie op het lab geadopteerd en waren nooit de moeilijkste als ik weer eens om hulp kwam vragen. Bedankt daarvoor.. hoewel ik bepaalde 'woorden van de week' liever niet had leren kennen!

Sander, verenigd door onze voorliefde voor mucus en lekker weer, bedankt voor alle tips en tricks zowel qua onderzoek als bij de gezelschapsspellen.

Kaatje, ik waardeer al je hulp en adviezen die je lang niet had hoeven geven, bedankt dat je zo behulpzaam bent geweest, volgende $\mathrm{x}$ als we samen fietsen zal ik je niet meer zo laten schrikken, beloofd!

Ruben, Kevin \& Marc: jullie hebben mij geïnspireerd als voorgangers, bedankt voor de samenwerking, sorry voor al het gespam per mail en whatsapp!

Marion, bedankt dat je me wegwijs hebt gemaakt in het microscopisch scoren van darmnaden, altijd fijn om samen een beetje te kletsen met drop en/of taart.

Het leuke aan een groot lab is dat je met heel veel verschillende mensen samenwerkt en dus ook veel verschillende dingen kan delen.. in willekeurige volgorde: Lieve Lori fijn dat als we samen eten er ook echt gekletst en geluisterd wordt, let's keep it up, maar dat gaat helemaal goedkomen nu we herenigd zijn en de aankomende 6 jaar alle cursussen samen gaan volgen, ons vol storten in de opleiding en nog veel WBS'sen onveilig gaan maken met onze dansmoves!

Lieve Briete \& Yvonne, mn favo Uns40 ladies, altijd fijn om even langs te huppelen bij jullie, even goed te kletsen over het leven en te dromen over de toekomst. Jasper \& Tim, jammer maar misschien ook wel beter dat jullie apart zaten al die tijd ;) Vic ik ga je nooit Billy noemen, hoop dat het goed met je blijft gaan op alle vlakken! Charlotte - altijd het goede voorbeeld, ook nu in de kliniek als mijn mentor ;) Altijd mijn go-to-girl als ik vragen heb over wat dan ook en dan ook nog gezellige eetsessies erbij! Givan, zullen we samen prijzen winnen voortzetten en vertalen naar goede CASH en ABSITE-scores? JM (bootypopping!), Liliane (mooi he, Afrikaanse billen), Toine, Rutger \& RJ (in 1 adem), Thiemo, 
Britt, Kirsten, Evelien, Milou (nu weer collega's! (-)), Rianne B, Timme, Paul, Niek, Robert, Martijn, en de nieuwe garde: Jacqueline, Marissa, Rianne V, Mirjam en Liyanne: succes en geniet ervan!

Dear Nina, Tiff, Jo, Berta \& Shane, together with Ron you came with the entire lab from Amsterdam to work at M4I, what a move! I'm sure there's so much cool stuff resulting from your experiments and I'm happy we could put some early data in this thesis!

Alle mensen van het CPV: bedankt voor de hulp beneden tijdens de studies! Sorry voor al die keren dat ik weer eens last-minute een ruimte wilde reserveren:-

Dear Sweden-people (that's how I call you), especially Gunnar, Karin, George \& Elisabeth, thank you so much for making my time at your lab so much fun and productive, it resulted in nice memories and a solid manuscript!

AnastomoSEAL-partners, especially Francesca, thank you for teaching me about your work, we had a really nice collaboration - unfortunately AL still exists and I loved visiting Gdansk, Oslo, Bordeaux \& Trieste with you as local guides!

Also thanks to Delibes, Vivaldi \& Tchaikovski, who were there when the working modus needed to be on full focus.

Lieve collega's van het Zuyderland; zowel de bazen, assistenten als verpleging: Ontzettend bedankt voor het warme welkom, de vele leermomenten, de grappen, de WBS, de buitendag en met name het vertrouwen in mij (in het bijzonder Ton, dankjewel!!), ik weet zeker dat er nog veel mooie momenten komen, zowel op het werk als daarbuiten, kijk mede door jullie enorm uit naar de toekomst.

Laten we proosten!

VVV'tjes: Marloes, Puck \& Deborah, volgens mij hebben jullie geen idee wat ik de afgelopen jaren heb gedaan, gelukkig maar want dan hoef ik bij jullie tenminste niet over werkdingen te praten haha. Wanneer is het weer kermis? Allerliefste Synt, ook jij bent een VVV, nu in HK, maar het Brabantse bloed stroomt toch waar het niet gaan kan dus maak ik me niet druk om de toekomst :;

Lieve Angela \& Marleen, vanaf de IDEE-week aan de VU tot het afstuderen bij de A-KO zijn jullie mijn medemeiden geweest :) Gek om zoveel jaren lief en leed met elkaar te delen en dat we nog steeds zulke goede vriendinnen zijn. Ben heel blij dat we elkaar zo goed begrijpen en dat we er zijn voor elkaar wanneer het echt nodig is! Superbedankt voor alle support en de gezelligheid! 
Lieve Tine \& Irene, hoewel het totaal niet logisch is om twee volledige leken als paranimfen te hebben zou ik het me niet kunnen voorstellen dat jullie niet achter me staan, om samen mee te shinen! Komisch dat we elkaars' wereldje qua werk niet echt begrijpen maar dat onze wereld samen de allerbeste is: vol wijntjes, goed eten en de drie-eenheid!

Lieve Bonma, 85 lentes jong en minstens zo eigenwijs. Voor iemand die ons allebei kent is het geen mysterie waar het gevoel voor drama bij mij vandaan komt. Ondanks dat het geen toneelschool in Maastricht is geworden hoop ik toch dat ik u een beetje trots heb gemaakt, andersom ben ik dat in ieder geval zeker!

Lieve Joost, ik ben blij dat het er inderdaad op lijkt dat wijsheid met de jaren komt en dat we elkaar niet meer het huis uit vechten. Jammer dat we elkaar niet heel vaak meer zien maar ben blij dat het allemaal zo goed gaat met je!

Liefste pap en mam, vanaf kleins af aan hebben jullie Joost en mij altijd gestimuleerd om het meeste uit het leven te halen en uitdagingen niet uit de weg te gaan. We mochten altijd onze eigen fouten maken en als het echt te bont werd, stonden jullie steevast klaar om in te grijpen of ons op te vangen. Ik kan jullie niet genoeg bedanken voor de onvoorwaardelijke steun, de kritische noot op z'n tijd, het vertrouwen in mij en de liefde die ik van jullie ontvang. Superbedankt!

Allerliefste Jules, ik had het echt niet getrokken zonder jou.. hoe suf het misschien soms ook lijkt, samen promoveren is echt chill.. muziekje aan, drankje erbij en gaan! Jij mijn figuren en statistiek checken, ik jouw teksten, goede deal! Vooral ook de broodnodige ontspanning tussendoor in wat voor vorm dan ook... 'Je geeft me de rust die ik als geen ander nodig heb, om te zijn wie ik ben'

Ben blij dat het nog steeds 'dikke prima' is tussen ons, ik hou van je! 


\section{List of publications}




\section{List of publications}

van den Brom CE, Bosmans JW, Vlasblom R, Handoko LM, Huisman MC, Lubberink M, Molthoff CF, Lammertsma AA, Ouwens MD, Diamant M, Boer C. Diabetic cardiomyopathy in Zucker diabetic fatty rats: the forgotten right ventricle. Cardiovasc Diabetol. 2010 Jun 15;9:25.

Vogels RR, van Barneveld KW, Bosmans JW, Beets G, Gijbels MJ, Schreinemacher $\mathrm{MH}$, Bouvy ND. Long-term evaluation of adhesion formation and foreign body response to three new meshes. Surg Endosc. 2015 Aug;29(8):2251-9.

Bosmans JW* Vogels RR*, van Barneveld KW, Verdoold V, van Rijn S, Gijbels MJ, Penders J, Breukink SO, Grijpma DW, Bouvy ND. A new Poly(1,3-trimethylene carbonate) film provides safe and effective adhesion reduction after major abdominal surgery in a rat model Surgery. 2015 Jun;157(6):1113-20.

Bosmans JW* Reisinger KW*, Uittenbogaart M, Alsoumali A, Poeze M, Sosef MN, Derikx JP. Loss of skeletal muscle mass during neoadjuvant therapy predicts postoperative mortality in esophageal cancer surgery Ann Surg Oncol. 2015 Dec;22(13):4445-52.

Bosmans JW, Jongen AC, Bouvy ND, Derikx JP. Colorectal anastomotic healing: why the biological processes that lead to anastomotic leakage should be revealed prior to conducting intervention studies. BMC Gastroenterol. 2015 Dec 21;15:180.

van Barneveld KW, Smeets BJ, Heesakkers FF, Bosmans JW Luyer MD, Wasowicz D, Bakker JA, Roos AN, Rutten HJ, Bouvy ND, Boelens PG. Beneficial Effects of Early Enteral Nutrition After Major Rectal Surgery: A Possible Role for Conditionally Essential Amino Acids? Results of a Randomized Clinical Trial. Crit Care Med. 2016 Jun;44(6):e353-61.

Bosmans JW, Moossdorff MD, Al-Taher M, van Beek L, Derikx JP, Bouvy ND. International consensus statement regarding the use of animal models for research on anastomoses in the lower gastrointestinal tract. Int J Colorectal Dis. 2016 May;31(5):1021-30.

Reisinger KW*, Schellekens DH*, Bosmans JW, von Meyenfeldt MF, Sastrowijoto P, Derikx JP, Grootjans J, Poeze M. Cyclooxygenase-2 is essential for colorectal anastomotic healing. Ann Surg. 2016 Apr 8. [Epub ahead of print]. 
Jongen AC, Bosmans JW, Kartal S, Lubbers T, Sosef M, Slooter GD, Stoot JH, van Schooten FJ, Bouvy ND, Derikx JP. Predictive Factors for Anastomotic Leakage After Colorectal Surgery: Study Protocol for a Prospective Observational Study (REVEAL Study). JMIR Res Protoc. 2016 Jun 9;5(2):e90.

Scognamiglio F, Travan A, Borgogna M, Donati I, Marsich E, Bosmans JW, Perge L, Foulc MP, Bouvy ND, Paoletti S. Enhanced bioadhesivity of dopamine-functionalized polysaccharidic membranes for general surgery applications. Acta Biomater. 2016 Oct 15;44:232-42.

Bosmans JW, Jongen AC, Boonen BT, van Rijn S, Scognamiglio F, Gijbels MJ, Bouvy ND. Comparison of three different application routes of butyrate to improve colonic anastomotic strength in rats. Int J Colorectal Dis. 2016 Dec 9. [Epub ahead of print].

Bosmans JW Jongen AC, Birchenough GM, Gijbels MJ, Nyström EE, Derikx JP, Bouvy ND, Hansson GC. Normal mucus composition is essential in colonic anastomotic healing in mice. In press, BJS 2017.

Bosmans JW Jongen AC, Vaes RD, Scognamiglio F, Borgogna, M Travan A, Marsich E, Gijbels MJ, Hoebers MT, Jocken JW, Donati I, Paoletti S, Bouvy ND. Caution should be taken when selecting glycerol as a plasticizer in biomaterials designed for intraabdominal use. Submitted.

Vakalopoulos KA, Bosmans JW, van Barneveld KW, Vogels RR, Boersema GS, Gijbels MJ, Jeekel J, Bouvy ND, Lange JF. Sealing insufficient colonic anastomoses with cyanoacrylate tissue adhesive: an in-vivo study. Submitted.

Bosmans JW*, Jongen AC*, Cillero-Pastor B, Ellis SR, Derikx JP, Bouvy ND, Heeren RM. New insights in colonic anastomotic healing through MALDI-mass spectrometry-imaging: results from a pilot study. In preparation. 
Curriculum vitae 


\section{Curriculum vitae}

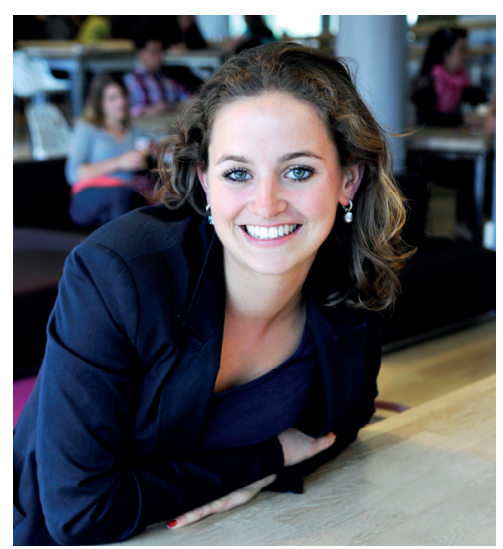

Anne-Claire Bosmans was born on the 3rd of November 1986 in Tilburg, the Netherlands. After graduating from the pre-university secondary school Theresialyceum in Tilburg in 2005, she went abroad to work as an au pair with a French family in Milan, Italy.

In 2006, she started her studies in Biomedical Sciences at the Vrije Universiteit in Amsterdam. It was during this period, while simultaneously studying Pharmaceutical Sciences, that helped spark her interest in scientific research which in turn motivated her to perform experiments in the laboratory. In 2009, she received her Bachelor's degree in Biomedical Sciences and started her research master (ArtsKlinisch Onderzoeker) at Maastricht University to become a clinical research physician. After a combined internship consisting of clinical work and scientific research at the department of General Surgery under supervision of Prof. Ernst van Heurn, she started her PhD project under the supervision of Prof. Nicole Bouvy and Dr. Joep Derikx, which resulted in this thesis. In February 2016, Anne-Claire started as a surgical resident at the Zuyderland Medical Centre in Sittard-Geleen under the supervision of Dr. Ton Hoofwijk. From the 1st of January 2016, she started her surgical training at the Zuyderland Medical Centre (supervision Dr. Meindert Sosef), which will be continued at the Maastricht University Medical Center under the supervision of Prof. Laurents Stassen. 
